\title{
Kationordnung und \\ Grenzflächeneffekte in stark \\ korrelierten Elektronensystemen
}

\section{DISSERTATION}

zur Erlangung des mathematisch-naturwissenschaftlichen Doktorgrades

„Doctor rerum naturalium"

der Georg-August-Universität Göttingen

im Promotionsstudiengang Physik der Georg-August University

School of Science (GAUSS)

vorgelegt von

Philipp Ksoll

aus Salzgitter

Göttingen, 2021 
Betreuungsausschuss:

apl. Prof. Dr. Vasily Moshnyaga,

I. Physikalisches Institut, Georg-August-Universität Göttingen

Prof. Dr. Christian Jooss,

Institut für Materialphysik, Georg-August-Universität Göttingen

apl. Prof. Dr. Michael Seibt,

IV. Physikalisches Institut, Georg-August-Universität Göttingen

Mitglieder der Prüfungskommission:

Referent: apl. Prof. Dr. Vasily Moshnyaga,

I. Physikalisches Institut, Georg-August-Universität Göttingen

Koreferent: Prof. Dr. Christian Jooss,

Institut für Materialphysik, Georg-August-Universität Göttingen

Weitere Mitglieder der Prüfungskommission:

apl. Prof. Dr. Michael Seibt,

IV. Physikalisches Institut, Georg-August-Universität Göttingen

Prof. Dr. Philipp Gegenwart,

Experimentalphysik VI, Universität Augsburg

Prof. Dr. Stefan Mathias,

I. Physikalisches Institut, Georg-August-Universität Göttingen

apl. Prof. Dr. Wolfram Kollatschny,

Institut für Astrophysik, Georg-August-Universität Göttingen

Tag der mündlichen Prüfung: 24. September 2021 
"Handle so, dass deine Handlungen ein echtes gedeihliches Weiterleben aller Lebewesen auf diesem Planeten möglich machen."

HANS JONAS 



\section{Inhaltsverzeichnis}

$\begin{array}{lr}\text { 1. Einleitung } & 1\end{array}$

2. Physikalische Grundlagen $\quad 7$

2.1. Übergangsmetalloxide in der Perowskitstruktur . . . . . . . . . 8

2.1.1. Kristall- und Elektronenstruktur . . . . . . . . 8

2.1.2. Austauschmechanismen . . . . . . . . . . . . . 16

2.2. Ordnungsphänomene in Doppelperowskiten . . . . . . . . . . . . . 20

2.2.1. Kristallstruktur und Kationordnung . . . . . . . . . . 20

2.2.2. Nickel und Kobalt basierte Doppelperowskite . . . . . . . . 27

2.3. Grenzflächenmagnetismus in oxidischen Heterostrukturen . . . . . 29

2.3.1. Kopplungseffekte an Grenzflächen . . . . . . . . . . . . 29

2.3.2. 3d/5d-Grenzflächen, exotische magnetische Phasen (Skyrmionen) . . . . . . . . . . . . . . . . . 34

3. Metallorganische Aerosol Deposition (MAD) 41

3.1. MAD Grundlagen und Aufbau . . . . . . . . . . . . . . . . . . . . 42

3.2. In-situ-Ellipsometrie . . . . . . . . . . . . . . . . . 46

3.2.1. Theoretische Betrachtung . . . . . . . . . . 46

3.2.2. Technische Realisierung . . . . . . . . . . . 50

4. Charakterisierung 53

4.1. Rasterkraftmikroskopie . . . . . . . . . . . . . . . . 54

4.2. Transmissionselektronenmikroskopie . . . . . . . . . . . 55

4.3. Röntgendiffraktometrie . . . . . . . . . . . . . . . . 57

4.4. Röntgenreflektrometrie . . . . . . . . . . . . . . . 60

4.5. Raman-Spektroskopie . . . . . . . . . . . . . . . . . . . 61

4.6. Magnetometrie . . . . . . . . . . . . . . . . 64

4.7. Messung des elektrischen Widerstandes . . . . . . . . . . . 66

5. Ergebnisse und Diskussion $\quad 69$

5.1. Grenzflächeneffekte, emergent Doppelperowskitphase und Kationordnung in $\left(\mathrm{LaNiO}_{3}\right)_{n} /\left(\mathrm{LaMnO}_{3}\right)_{n}$-Heterostrukturen . . . . . 72

5.1.1. Grenzflächeneffekte zwischen $\mathrm{LaNiO}_{3}$ und $\mathrm{LaMnO}_{3}$. . . 72

5.1.2. Kationordnung in $\mathrm{LaNiO}_{3} / \mathrm{LaMnO}_{3}$ - Heterostrukturen mittels systematischer Reduzierung der Einzelschichtdicke . . 93 
5.2. Kationordnung in 3d-Doppelperowskiten und Heterostrukturen durch stöchiometrischen Lagenwachstum . . . . . . . . . . . . . . 97

5.2.1. $\mathrm{La}_{2} \mathrm{CoMnO}_{6}$ und $\mathrm{LaCoO}_{3} / \mathrm{LaMnO}_{3}$ - Heterostrukturen . . 97

5.2.2. $\mathrm{Sr}_{2} \mathrm{TiRuO}_{6}$ und $\mathrm{SrTiO}_{3} / \mathrm{SrRuO}_{3}$ - Heterostrukturen . . . . 106

5.3. Grenzflächeneffekte und Kationordnung in 3d/5d-Übergittern . . . 119

5.3.1. Grenzflächeneffekte und Kationordnung in $\mathrm{SrMnO}_{3} / \mathrm{SrIrO}_{3}$ -

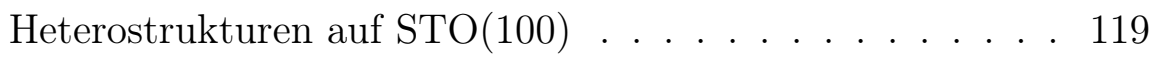

5.3.2. Grenzflächeneffekte in $\mathrm{La}_{0.7} \mathrm{Sr}_{0.3} \mathrm{MnO}_{3} / \mathrm{SrIrO}_{3}$ - Heterostrukturen . . . . . . . . . . . . . . . . . 134

$\begin{array}{ll}\text { 6. Zusammenfassung und Ausblick } & 139\end{array}$

A. Anhang

B. Literaturverzeichnis

Danksagung

Lebenslauf

178

Publikationen 


Kapitel 1

\section{Einleitung}

Die komplexe Wechselwirkung zwischen Spin-, Gitter- und Elektronensystemen führt in Übergangsmetalloxiden (TMO, engl.: transition metal oxides) zu einer großen Vielzahl an exotischen physikalischen Phänomenen. Zu einem der prominentesten Beispiele zählt die Entdeckung des kolossalen Magnetowiderstandes (CMR, engl.: colossal magnetoresistance) an gemischtvalenten Manganoxiden [1,2] oder auch die Beobachtung von Hochtemperatursupraleitung im Jahre 1950 [3,4]. Ein besonderer Fokus der Forschung liegt dabei auf der Unterklasse der Perowskitoxide mit der Summenformel $\mathrm{ABO}_{3}$, deren A- und B-Plätze mit den meisten metallischen Elementen besetzt werden können und systematische Dotierungen $\left(\mathrm{A}_{1-x} \mathrm{~A}_{x}{ }_{x} \mathrm{BO}_{3}\right.$ und $\mathrm{AB}_{1-x} \mathrm{~B}^{\prime}$ ${ }_{x} \mathrm{O}_{3}$ ) erlauben. Dabei spielt neben der stöchiometrischen Verteilung auch die Ordnung der Kationen innerhalb des Kristalls eine ausschlaggebende Rolle für die elektrischen und magnetischen Eigenschaften des Materials. Dies wird insbesondere bei den sogenannten Doppelperowskiten (DP) mit der Summenformel $\mathrm{A}_{2} \mathrm{BB}^{\prime} \mathrm{O}_{6}$ deutlich, deren B-Platz durch zwei unterschiedliche Kationen im Verhältnis 1:1 besetzt ist. Der Grad der B-Platzordnung bestimmt maßgeblich die Kristallstruktur und die Wechselwirkung zwischen den jeweiligen B-Kationen und wird durch die Wachstumsbedingungen und die Wahl der Kationen bestimmt [5,6]. Verbunden mit unumgänglichen Kristalldefekten verhindern diese in den meisten Fällen eine vollständige B-Platzordnung und beeinflussen die Eigenschaften des Doppelperowskits. Beispiele dafür sind das halb-metallische DP $\mathrm{Sr}_{2} \mathrm{MoFeO}_{6}$, dessen Spin- 
Polarisation an der Fermikante mit zunehmender B/B'-Unordnung drastisch abnimmt [7] oder das DP $\mathrm{Pd}_{2} \mathrm{ScTaO}_{6}$, dessen dielektrisches Verhalten durch die B-Platzordnung vorgegeben wird $[5,8]$.

Diese Beispiele zeigen, dass die Herstellung kationgeordneter korrelierter Oxidfilme sowohl für die Grundlagenforschung als auch für Anwendungen ein wesentlicher Durchbruch sein wird. Korrelationseffekte in einer unordnungsfreien kondensierten Materie zu untersuchen, motiviert die wissenschaftliche Langzeitvision. Besonders wichtige Auswirkungen wird die Kationordnung in den Bereichen der Untersuchung klassischer und Quantenphasenübergänge, niedrigdimensionaler Systeme und nanoskaliger Physik haben [9]. Unter dem Gesichtspunkt der Anwendung besteht eine große Motivation, die treibenden Kräfte der Ordnung tiefgehender zu verstehen und eine Methode zu entwickeln, mit Hilfe derer man eine hohe B-Platzordnung zuverlässig herstellen kann.

Ein Vorteil der Perowskitstruktur besteht darin, dass sich durch ihre ähnliche Gitterstruktur unterschiedliche Perowskitoxide kombinieren und epitaktische Heterostrukturen herstellen lassen, die völlig neue physikalische Phänomene an ihren Grenzflächen hervorbringen. Beispiele sind Ladungstransfer, strukturelle Kopplungen, orbitale Polarisationen und Rekonstruktion. Die Eigenschaften der daraus resultierenden Grenzfläche mit ihrem neuartigen Grenzflächeneffekten unterscheiden sich grundlegend von den Eigenschaften der beteiligten Einzelschichtsysteme. Das Thema bildet somit ein eigenes, weites Feld der modernen Festkörperphysik [10]. So wird bisher davon ausgegangen, dass sich beispielsweise aus polaren Diskontinuitäten an der Grenzfläche der zwei isolierenden Schichten $\mathrm{LaAlO}_{3}$ und $\mathrm{SrTiO}_{3}$ ein zweidimensionales Elektronengas ergibt [11]. In Heterostrukturen aus $\mathrm{LaMnO}_{3} / \mathrm{SrMnO}_{3}$ wird hingegen eine ferromagnetische Hochtemperaturphase als Ergebnis eines Ladungstransportes (CT, engl: charge transfer) aus der elektronenreichen $\mathrm{LaMnO}_{3}$-Schicht in die elektronenarme $\mathrm{SrMnO}_{3}$ Schicht an der Grenzfläche beobachtet [12].

Die genannten Beispiele gehören zu der Klasse der 3d-TMO, deren Physik auf einer starken Coulombwechselwirkung und daraus entstehenden elektronischen Korrelationen basiert. In den $4 \mathrm{~d}-$ und $5 \mathrm{~d}-$ Übergangsmetalloxiden 
wird hingegen nur ein schwach korreliertes elektronisches Verhalten erwartet, da die intraatomare Coulombabstoßung aufgrund der zunehmenden radialen Ausdehnung der d-Orbitale bei aufsteigender Ordnungszahl zunehmend schwächer wird. Im Gegensatz dazu folgt die relativistische SpinBahn-Kopplung (SOC, engl.: spin orbit coupling) dem entgegengesetzten Trend und erreicht in einigen schweren $5 \mathrm{~d}$-Verbindungen eine vergleichbare Größenordnung wie die Coulombwechselwirkung. So führt in iridiumbasierenden 5d-TMO $\mathrm{Sr}_{2} \mathrm{IrO}_{4}$ die SOC zur Bildung eines halb gefüllten Bandes mit effektiven Gesamtdrehimpuls $J_{\text {eff }}=1 / 2$ [13]. Die Kombination aus starker elektronischer Korrelation und SOC bietet ein breites Feld an neuartigen Phänomenen, die die Herstellung neuartiger $3 \mathrm{~d} / 5 \mathrm{~d}-$ und auch 4d/5d-Grenzflächen ermöglicht. So können derartige Grenzflächen zur Bildung des sogenannten topologischen Halleffektes (THE) führen, der über die Dzyaloshinskii-Moriya-Wechselwirkung (DM) mit der Bildung von nanoskäligen topologischen Defekten, namentlich Skyrmionen, in Verbindung gebracht wird [14-26]. Dies ist derzeit Gegenstand aktiver Forschung. Skyrmionen sind nano-wirbelartige Spinkonstrukte, die in einer ferromagnetischen Umgebung selbst bei kleinen Größen sehr stabil sind, weshalb sie als vielversprechende zukünftige Informationsträger gelten.

Ermöglicht wird die Herstellung dieser Heterostrukturen durch die gängigen und stets verfeinerten Depositionsmethoden. Die Dünnfilme, die in dieser Arbeit vorgestellt werden, wurden mittels der Metallorganischen Aerosol Deposition (MAD) hergestellt. Im Gegensatz zu den konventionellen Depositionsmethoden - Molekularstrahlepitaxie (MBE, engl.: molecular beam epitaxie), der gepulsten Laserdeposition (PLD, engl.: pulsed laser deposition) und dem Sputtern - arbeitet die MAD bei atmosphärischen Bedingungen $\left(p_{\mathrm{O}_{2}}=0,2\right.$ bar $)$. Der Atmosphärendruck in der Anlage verhindert den Einsatz der oberflächensensitiven in-situ-Wachstumskontrolle über die Beugung hochenergetischer Elektronen bei Reflexion (RHEED, engl.: reflection high-energy electron diffraction). Aus diesem Grund ist die MAD-Anlage mit der optischen Ellipsometrie ausgestattet, die aufgrund ihrer hohen Empfindlichkeit auf die elektronischen Eigenschaften der deponierenden Materialien detaillierte Rückschlüsse auf die Wachstumsmode, aber auch auf die 
Verteilung der Elektronen auf den Übergangsmetallionen, erlaubt [12, 27]. So konnten bereits Rückschlüsse auf die charakteristische Länge des Ladungstransportes in $\mathrm{LaMnO}_{3} / \mathrm{SrMnO}_{3}$-Heterostrukturen gezogen werden, die Aufschluss über das Auftreten der Hochtemperaturphase liefern [12].

Die jeweiligen Depositionsmethoden werden daran gemessen, mit welcher Qualität sie die Dünnfilme herstellen. Um den Ordnungsgrad der Doppelperowskite weiter zu verbessern, wird die MAD im Rahmen der vorliegenden Arbeit weiterentwickelt. Dafür werden die Filme durch eine lagenweise Deposition der Einzelschichtsysteme (LbL, engl.: Layer-by-Layer growth mode) gewachsen, d.h. durch sequentielles Wachstum der einzelnen $\mathrm{ABO}_{3} / \mathrm{AB}^{\prime} \mathrm{O}_{3} / \mathrm{ABO}_{3} \ldots$ Schichten. Eine solche Technik kann ein genaueres Verständnis der treibenden Kräfte der B-Platzordnung liefern und eine zuverlässige Wachstumskontrolle auf der Ebene einer einzelnen Atomschicht bieten. Damit könnte man kationgeordnete Filme aus stark korrelierten Oxiden züchten.

Aufbau der Arbeit Zu Beginn dieser Arbeit sollen in Kapitel 2 die theoretischen Grundlagen für die Betrachtung der untersuchten Dünnfilme erläutert werden, bevor die experimentellen Gegebenheiten in Kapitel 3 (MADWachstum) und 4 (Charakterisierung) beschrieben werden. Dabei soll in einem gesonderten Kapitel die Metallorganischen Aerosol Deposition mit der in-situ-Ellipsometrie hervorgehoben werden.

Den Hauptteil der vorliegenden Arbeit bildet das Kapitel 5 über die hergestellten Dünnfilme und die daraus resultierenden Ergebnisse. Dabei werden im ersten Teil Heterostrukturen aus den zwei 3d-Systemen $\mathrm{LaNiO}_{3}$ und $\mathrm{LaMnO}_{3}$ auf (111) orientiertem $\mathrm{SrTiO}_{3}$ hergestellt, bei denen die Einzelschichtdicke systematisch reduziert wird, um schließlich bei einer Einzelschichtdicke von einer Monolage eine kontrollierte B-Platzordnung zu erzielen. Die kontinuierliche Reduzierung der Schichtdicke der Einzelschichtsysteme und der damit einhergehende Übergang zum DP $\mathrm{La}_{2} \mathrm{NiMnO}_{6}$ offenbart einen tiefen Einblick in den vorliegenden Grenzflächenmagnetismus. Dieser soll der zusammen mit der erzielten Kationordnung vorgestellt und untersucht werden. 
Die gewonnenen Erfahrungen werden genutzt und auf weitere Materialsysteme aus $\mathrm{LaCoO}_{3} / \mathrm{LaMnO}_{3}$ und $\mathrm{SrTiO}_{3} / \mathrm{SrRuO}_{3}$ angewandt. Hierbei wird jedoch die B-Platzordnung nicht durch die systematischen Reduzierung der Einzelschichtdicke erzielt, sondern durch eine systematische Variation der Lagenstöchiometrie. Die hergestellten Proben werden auch hier mit der klassischen Wachstumsmethode (SoA, engl.: State of the Art) verglichen und die treibenden Kräfte zur Bildung der B-Platzordnung weiter untersucht.

Motiviert durch die Suche nach dem topologischen Halleffekt werden die Untersuchungen auf Heterostrukturen mit dem 5d-TMO $\mathrm{SrIrO}_{3}$ erweitert. In Kombination mit $\mathrm{SrMnO}_{3}$ wird ähnlich wie in dem System aus $\mathrm{LaNiO}_{3}$ und $\mathrm{LaMnO}_{3}$ die Schichtdicke der einzelnen Systeme systematisch reduziert, um die Kationordnung herzustellen. Anders als in den Systemen zuvor werden die Proben hier auf $\mathrm{SrTiO}_{3}$-Substraten in (100)-Orientierung hergestellt. Neben den Grenzflächeneffekten wird somit die künstliche BPlatzordnung in (100)-Richtung untersucht und mit dem natürlichen SoAWachstum verglichen. Abschließend werden die Untersuchungen des THE auf Heterostrukturen aus $\left(\mathrm{La}_{0.7} \mathrm{Sr}_{0.3} \mathrm{MnO}_{3}\right)_{n} /\left(\mathrm{SrIrO}_{3}\right)_{2}$ mit $\mathrm{n}=4$ und 6 erweitert. Die Proben werden auf ihren Grenzflächenmagnetismus hin untersucht, dessen Erkenntnisse die Grundlage für weitere Forschungsunternehmungen bilden können. 



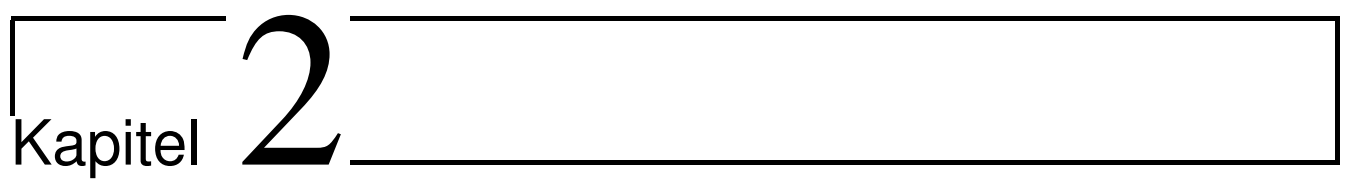

\section{Physikalische Grundlagen}

Wie in der Einleitung bereits erwähnt, zeigen sowohl die einzelnen TMO als auch die Kombination der TMO an deren Grenzfläche eine große Fülle an magnetischen und elektrischen Eigenschaften. Diese beruhen auf einer Vielzahl von physikalischen Phänomenen, die ihren Ursprung in der Wechselwirkung aus Spin-, Gitter- und Elektronensystem haben. Ein grundlegendes theoretisches Verständnis für diese Wechselwirkungen und den daraus resultierenden Konsequenzen für die Eigenschaften der Dünnfilme soll zunächst für die Perowskit-Übergangsmetalloxide erarbeitet werden. Diese bilden das Fundament der vertieften Untersuchungen an Doppelperowskiten und TMO-Heterostrukturen.

Anschließend wird ausführlicher auf die Klasse der Doppelperowskite eingegangen und der Einfluss der Kationordnung auf deren strukturelle, elektrische und magnetische Eigenschaften der untersuchten Doppelperowskitsysteme aus $\mathrm{La}_{2} \mathrm{NiMnO}_{6}$ und $\mathrm{La}_{2} \mathrm{CoMnO}_{6}$ erläutert.

Das nächste Unterkapitel widmet sich dem Grenzflächenmagnetismus und den damit verbundenen Grenzflächeneffekten, die sich bei der Kombination der Einzelkomponenten der vorgestellten Doppelperowskiten ergeben. Dabei wird zu Beginn der Fokus auf die physikalischen Phänomene an Grenzflächen zwischen unterschiedlichen 3d-TMO gelegt, bevor das Bild auf $5 \mathrm{~d}-$ TMO und die Grundlagen des topologischen Halleffektes erweitert wird. 


\section{1. Übergangsmetalloxide in der Perowskitstruktur}

Elemente, die unvollständig besetzte d-Orbitale besitzen, werden als Übergangsmetalle bezeichnet (Grün eingefärbte Felder im Periodensystem in Abbildung 2.1), die in Verbindung mit Sauerstoff Übergangsmetalloxide bilden. Zusammen mit seltenen Erden und/oder Erdalkalimetallen können diese Materialsysteme in der Perowskitstruktur wachsen, die im Folgenden vorgestellt werden soll.

\subsubsection{Kristall- und Elektronenstruktur}

Kristallstruktur Die Übergangsmetalle besetzten in der $\mathrm{ABO}_{3}$-Perowskitstruktur die B-Plätze und sind umgeben von sechs Sauerstoffanionen $\mathrm{O}^{-2}$, die zusammen ein $\mathrm{BO}_{6}$-Oktaeder bilden. Die A-Plätze werden durch Kationen belegt (seltene Erden: La, Pr, Tb... und/oder Erdalkalimetalle: Sr, Ca, Ba...), deren Valenz die Elektronenanzahl auf dem B-Kation bestimmt. Ne-

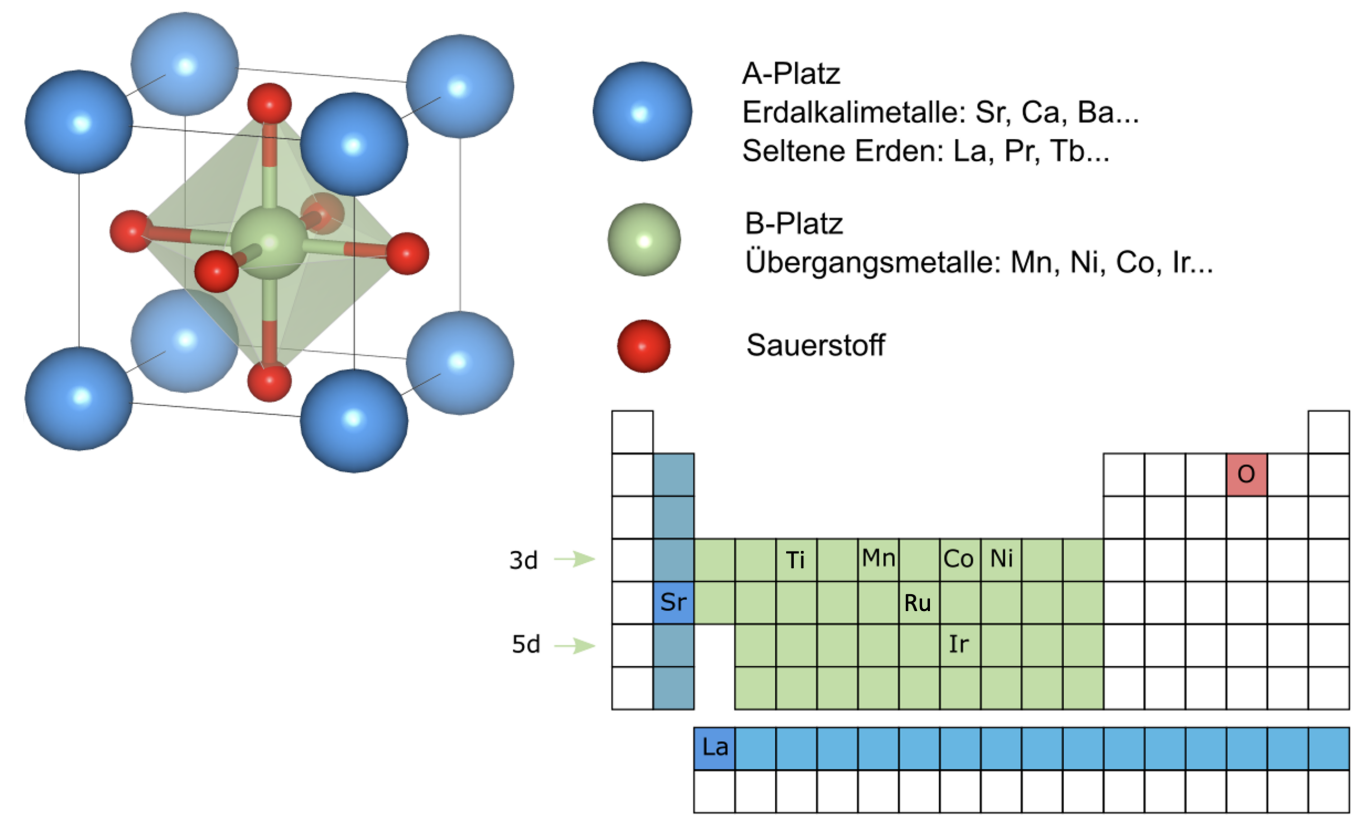

Abbildung 2.1.: Darstellung einer Einheitszelle der kubischen Perowskitstruktur (angefertigt mit [28]) und das Periodensystem. Die in dieser Arbeit verwendeten Elemente sind zusätzlich im Periodensystem eingetragen. 
ben der Valenz spielt der B-O-B Winkel zwischen den B-Kationen eine Rolle für die magnetischen Eigenschaften, der im Wesentlichen durch die relative Größe der A- und B-Kationen zueinander bestimmt wird. Leichte Veränderungen des Winkels führen bereits zu Abweichungen der idealen kubischen Struktur (Raumgruppe Pm3m). Diese Abweichung kann mithilfe des Goldschmidt'schen Toleranzfakotors $t$ über die A-O- und B-O- Bindungslängen charakterisiert werden: $t=r_{A-O} /\left(r_{B-O} \sqrt{2}\right)[5,6,10,29]$. Für die ideale kubische Struktur ergibt sich der Wert $t=1$. Allerdings zeigt die Mehrheit der Perowskitoxide eine Abweichung, die sich im Bereich 0,71<t<1 befindet und durch kooperative Verkippungen der Sauerstoffoktaeder ausgeglichen wird, wie in Abbildung 2.2 dargestellt [30]. Bei relativ kleinen Verzerrungen führt diese Situation zu pseudokubischen Strukturen mit orthorhombischer, tetragonaler oder rhomboedrischer Symmetrie. Die oktaedrischen Rotationen verändern die B-O-B-Bindungswinkel und in geringerem Maße die Bindungslängen. Aufgrund mehrerer konkurrierender magnetischer Wechselwirkungen können bereits relativ kleine Verzerrungen das Vorzeichen und die Stärke der durch diese Bindungen vermittelten Austauschwechselwirkungen beeinflussen. Unterschreitet der Toleranzfaktor einen gewissen Wert $t \approx 0,71$ wird die Perowskitstruktur instabil und andere Strukturtypen wie Korund oder Ilmenit bilden sich aus [30,31]. Währenddessen wird für gewisse AKationen ein Toleranzfaktor $t>1,02$ erzielt, ab dem die Verspannung durch Verdrehung und Verkippung der Oktaeder nicht mehr ausgeglichen werden kann und zu einer hexagonalen Struktur führt [6,31].
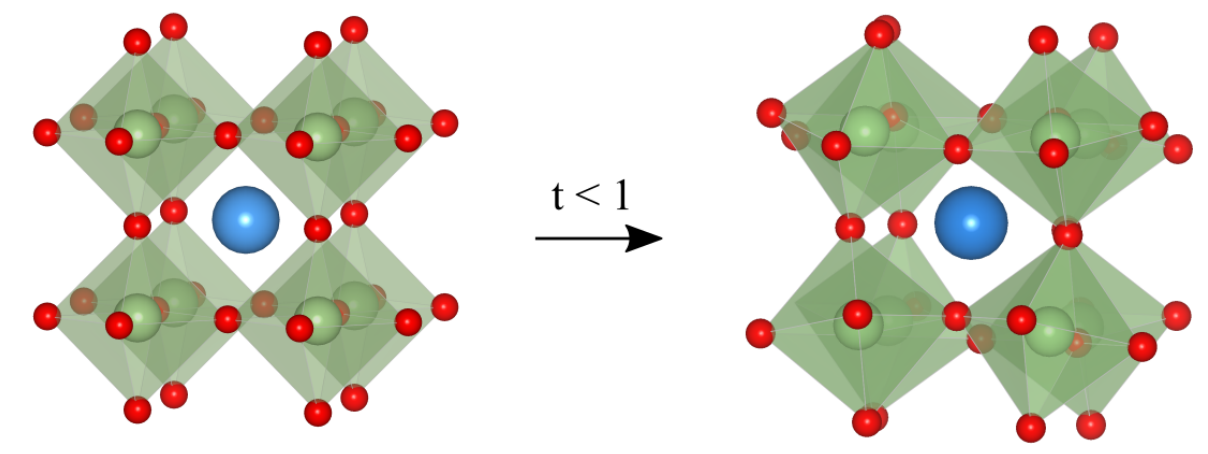

Abbildung 2.2.: Struktur der $\mathrm{ABO}_{3}$ Perovskit-Oxide in der idealen kubischen Struktur mit $t=1$ und mit kooperativer Verkippung der Oktaeder mit $t<1$. Farbgebung wie in Abb. 2.1. 
Elektronenstruktur der 3d-Perowskite In einem isolierten Übergangsmetallion befinden sich die äußeren fünf 3d-Orbitale auf einem Energieniveau. In der Perowskitstruktur ist jedoch das Übergangsmetallion auf dem B-Platz umgeben von den Sauerstoffoktaedern (vergleiche Abbildung 2.1). Durch die Coulombwechselwirkung mit den $\mathrm{O}^{-2}-2 \mathrm{p}-$ Orbitalen kommt es zu einer Aufhebung der Entartung der 3d-Zustände, der sogenannten Kristallfeldaufspaltung. Die drei Orbitale $d_{x y}, d_{y z}$ und $d_{x z}$ werden dabei energetisch abgesenkt und als $t_{2 g}$-Orbitale zusammengefasst, während die beiden übrigen Orbitale $d_{z^{2}}$ und $d_{x^{2}-y^{2}}$ energetisch erhöht und als $e_{g}$-Orbitale zusammengefasst werden $\left(\Delta_{C F} \approx 1-2 \mathrm{eV}\right)[32,33]$.

Die dreifache Entartung des $t_{2 g}$ bzw. die doppelte Entartung des $e_{g}$-Bandes kann weiter aufgehoben werden, wenn es zu einer tetragonalen Verzerrung der Sauerstoffoktaeder kommt. Dies kann sowohl durch den sogenannten Jahn-Teller-Effekt als auch durch biaxiale Verspannungen verursacht werden. Der Jahn-Teller-Effekt tritt auf, wenn es durch die Verzerrung der Oktaeder zu einem Gewinn der Coulombenergie kommt und kann in Systemen mit vier 3d-Elektronen zu einer High-Spin-Konfiguration führen, wie am Beispiel von $\mathrm{Mn}^{3+}$ in Abbildung 2.3 a) dargestellt [32-34]. Biaxiale Verspannungen entstehen hingegen durch voll verspanntes epitaktisches Wachstum

a)

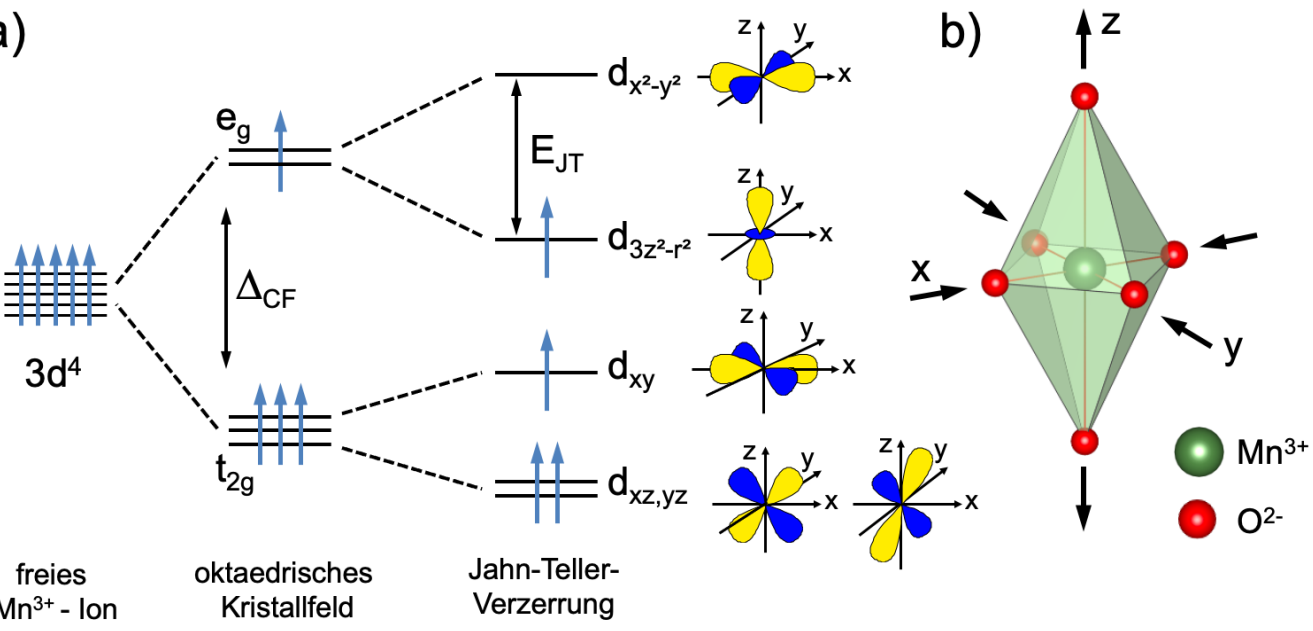

Abbildung 2.3.: a) Kristallfeldaufspaltung und Jahn-Teller-Effekt der $\mathrm{Mn}^{3+}-3 \mathrm{~d}$-Orbitale und b) Jahn-Teller-Verzerrung des $\mathrm{MnO}_{6}$-Oktaeders. Mit Erlaubnis von Elsevier nach [33]. 
eines Dünnfilmes auf einem Substrat mit positiver bzw. negativer Gitterfehlanpassung, was in Kapitel 2.3.1 näher erleuchtet wird.

Die Entartung im $t_{2 g}$-Niveau kann auch durch die Spin-Bahn-Kopplung aufgehoben werden. Für die hier betrachteten 3d-Übergangsmetalle ist die Spin-Bahn-Kopplung jedoch relativ schwach [35] (Näheres im nächsten Abschnitt), sodass diese im Falle der 3d-Oxide vernachlässigt wird und sich das magnetische Moment aus $g \mu_{B} S$ ergibt, mit dem Landé-Faktor $g \approx 2, S$ dem Spin der 3d-Elektronen und $\mu_{B}$ dem Bohr'schen Magneton.

Die Energie, die benötigt wird, um ein Orbital mit zwei Elektronen mit antiparallelen Spins zu besetzten, wird als Hund'sche-Energie $J_{H}$ bezeichnet und bestimmt die Besetzung der Bänder. Ist die Hund'sche-Energie größer als das Kristallfeld, $J_{H}>\Delta_{C F}$, spricht man von der High-Spin-Konfiguration (HS): die $e_{g}$-Orbitale werden mit Elektronen besetzt, bevor ein $t_{2 g}$-Orbital mit einem zweiten Elektron besetzt wird. Dies wird für die meisten $3 \mathrm{~d}-$ Übergangsmetalloxide beobachtet, unter anderem Mangan [31-33]. Ausnahmen sind Nickelate $\left(3 \mathrm{~d}^{7}\right.$-Konfiguration für $\left.\mathrm{Ni}^{3+}\right)$, für die sich ein Low-SpinZustand (LS) ergibt $\left(J_{H}<\Delta_{C F}\right)$ [36], und Cobaltate, bei denen sich für das $\mathrm{Co}^{3+}$-Kation ( $3 \mathrm{~d}^{6}$-Konfiguration) neben dem HS- und LS- ein IntermediateSpin-Zustand (IS) ausbilden kann $\left(J_{H} \approx \Delta_{C F}\right)[31,37]$.

Spin-Bahn Kopplung in 5d-Iridaten Spin-Bahn-Kopplung (SOC) ist die Wechselwirkung zwischen der Orbitalbewegung eines Elektrons und seinem Spin, die sich aus der relativistischen Quantenmechanik ergibt [38]. Für ein einzelnes Elektron in einem Atom mit dem sphärischem Potential $V(r)=$ $\frac{Z e}{4 \pi \epsilon_{0}} \frac{1}{r}$ ist die Spin-Bahn-Kopplung gegeben über [38,39]:

$$
H_{S O C}=\xi \boldsymbol{l} \cdot \boldsymbol{s}, \quad \text { mit } \quad \xi=\frac{1}{8 \pi m_{e}^{2} c^{2} \epsilon_{0}} \frac{Z e^{3}}{r^{2}}
$$

woraus sich folgende Aufspaltung ergibt [39]:

$$
E_{S O C}=\frac{Z^{4} e^{2} \hbar^{2}}{16 \pi m_{e}^{2} c^{2} \epsilon_{0}}\left(\frac{j(j+1)-l(l+1)-s(s+1)}{l(l+1 / 2)(l+1) a_{0}^{3} n^{3}}\right),
$$

mit: $\boldsymbol{l}$ Bahndrehimpuls, $\boldsymbol{s}$ Spindrehimpuls, $Z$ der Ordnungszahl, $e$ der Elektronenladung, $\epsilon_{0}$ der dielektrischen Konstanten, $r$ dem radialen Abstand, $\hbar$ 
der Plank'schen Konstante, $c$ der Lichtgeschwindigkeit, $m_{e}$ der Elektronenmasse, $a_{0}$ dem Bohr'schen Radius, $n$ der Hauptquantenzahl und letztlich $j, l$ und $s$ den Quantenzahlen für den Gesamt-, Bahn- und Spindrehimpuls. Für ein Mehrelektronensystem ergibt sich der Hamiltonoperator aus der Summe aller Elektronen:

$$
H_{S O C}=\sum_{i} \xi_{i} l_{i} \cdot s_{i} \approx \lambda L \cdot S
$$

mit $L$ dem Gesamtbahn- und $S$ dem Gesamtspindrehimpuls. Aus diesen Gleichungen wird ersichtlich, dass die Spin-Bahn-Kopplung proportional zur vierten Potenz der Ordnungszahl ist, $\lambda \propto Z^{4}$, weshalb für $3 \mathrm{~d}-$ Systeme die Spin-Bahn-Kopplung vernachlässigbar gering ausfällt, während sie auf $5 \mathrm{~d}$-Systeme einen erheblichen Einfluss hat. So beträgt beispielsweise die SOC-Konstante $\lambda$ für Kupfer mit der Ordnungszahl $Z=29$ moderat $\lambda \approx$ $0,01 \mathrm{eV}$ [40], während sie für Iridium mit der Ordnungszahl $Z=77$ bereits $\lambda \approx 0,5 \mathrm{eV}$ beträgt [41] und deshalb mit betrachtet werden muss.

In $\mathrm{SrIrO}_{3}$ (inklusive der Ruddlesden-Popper-Verbindungen $\operatorname{Sr}_{n+1} \mathrm{Ir}_{n} \mathrm{O}_{3 n+1}$ ) ist das Iridium tetravalent und nimmt die $5 \mathrm{~d}^{5}$-Konfiguration an. Aufgrund

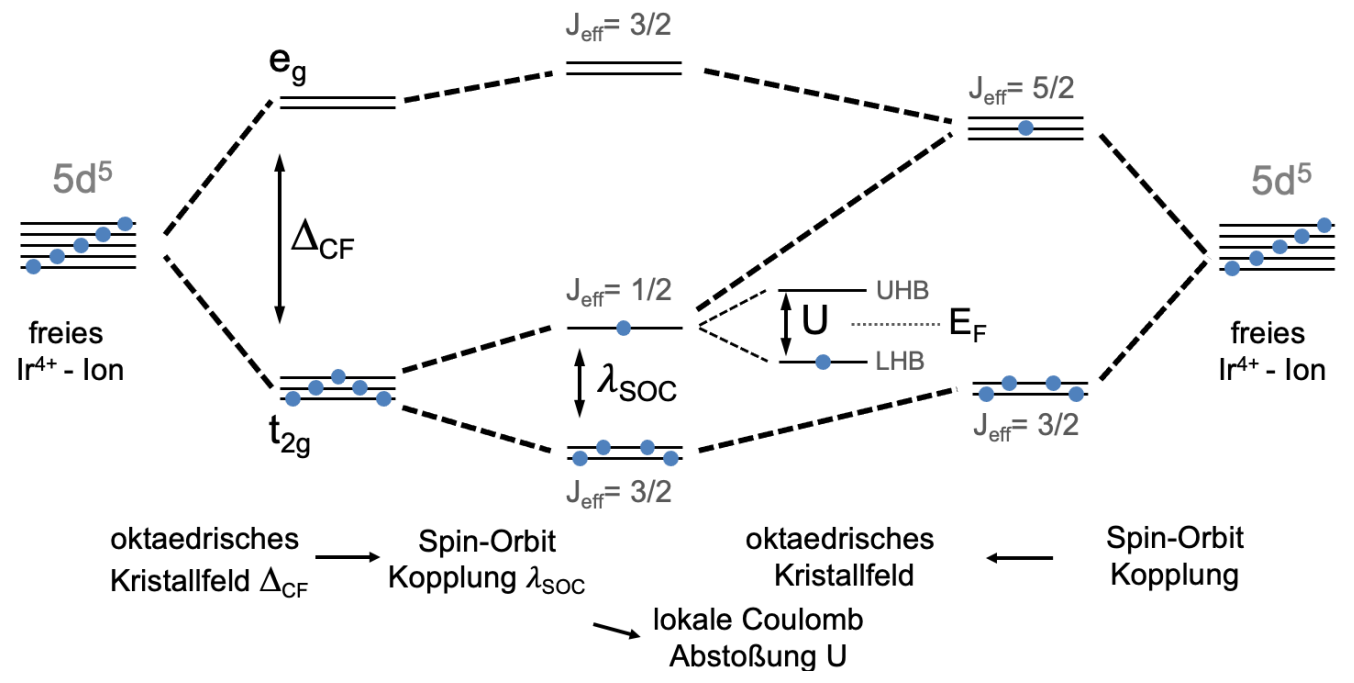

Abbildung 2.4.: Energieaufspaltung des 5d-Bandes infolge der Kristallfeldaufspaltung und der Spin-Bahn-Kopplung am Beispiel des $\mathrm{Ir}^{4+}-5 \mathrm{~d}^{5}$-Ions. Mit Erlaubnis nach der Abbildung aus [42]. 
des großen Überlappens der 5d--Orbitale des Iridiums und der 2p- Orbitale des Sauerstoffions überwiegt die Kristallfeldaufspaltung gegenüber der Hund'schen-Kopplung, sodass das Iridium den LS-Zustand annimmt und somit ausschließlich das $t_{2 g}$-Band besetzt [13], wie in Abbildung 2.4 dargestellt.

Aufgrund der SOC wird schließlich das $t_{2 g}$-Niveau weiter aufgeteilt in ein einfach besetztes Niveau mit Gesamtdrehimpuls $J_{\text {eff }}=1 / 2$ und ein vierfach besetztes Niveau mit $J_{\text {eff }}=3 / 2$. Dies reduziert das System effektiv auf ein halb gefülltes $J_{\text {eff }}=1 / 2$ Einzelbandsystem [13]. Dabei wird das finale Bandschema, das in der Mitte der Abbildung 2.4 dargestellt ist, unabhängig davon erreicht, ob zunächst die Kristallfeldaufspaltung und dann die SOC angewandt wird (Fall: von links in die Mitte) oder aber zunächst die SOC und dann die Kristallfeldaufspaltung (Fall: von rechts in die Mitte) [42]. Die Coulombabstoßung $U$ führt zu einer weiteren Aufspaltung des einfach besetzten Niveaus in ein oberes Hubbard-Band und ein unteres HubbardBand, wie im Folgenden beschrieben wird.

Hubbard-Modell Aufgrund des geringen Überlappens benachbarter Orbitale muss zur Beschreibung des Verhaltens der Elektronen ihre Wechselwirkung untereinander mit berücksichtigt werden. Diese Wechselwirkung wird im Hubbard-Modell eingebunden, bei dem Elektronen zwischen den
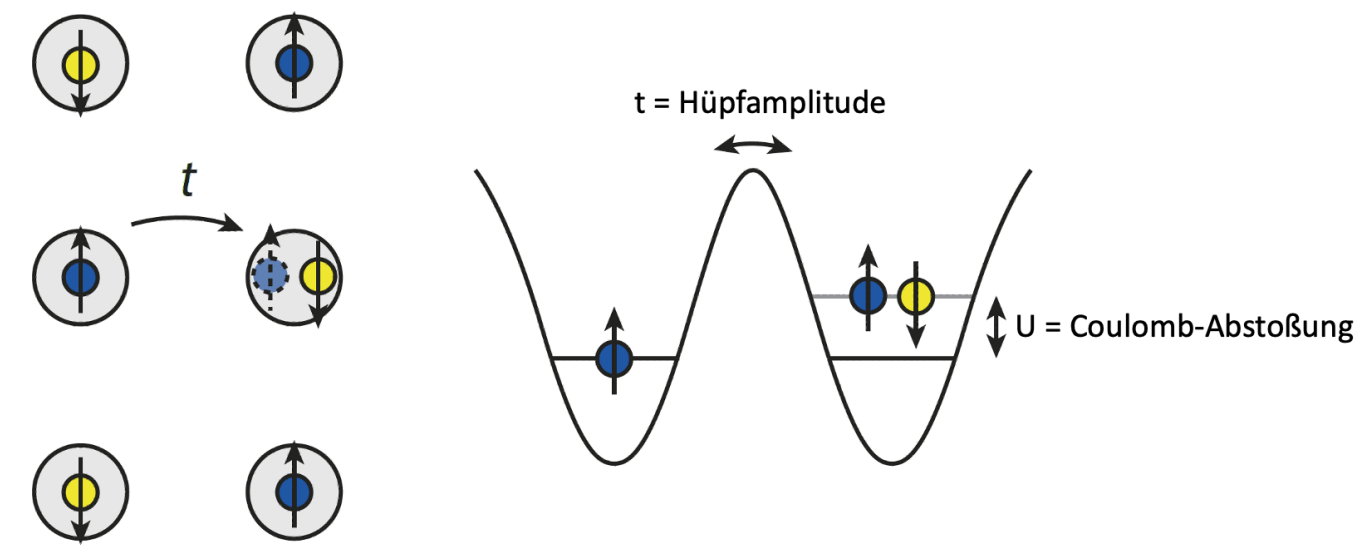

Abbildung 2.5.: Bildliche Darstellung des Hubbard-Modells auf einem zweidimensionalen quadratischen Gitter mit Darstellung der Potentiallandschaft. Nach dem Schema aus [23]. 
B-Plätzen „hüpfen” können. Dabei wird ihre Coulombwechselwirkung mit berücksichtigt. Hüpft nun ein Elektron auf ein benachbartes Ion, auf dessen verfügbaren Niveau bereits ein Elektron sitzt, ist dies mit dem Aufbringen einer Coulombenergie $U$ verbunden, während die Delokalisierung der Elektronen durch den Hüpfprozess zu einem Gewinn an kinetischer Energie führt, was über die Hüpfamplitude $t$ charakterisiert wird, wie in Abbildung 2.5 dargestellt. Unter Berücksichtigung beider Effekte kann der HamiltonOperator des eindimensionalen Hubbard-Modells geschrieben werden als:

$$
H_{H U B}=-t \sum_{\langle i, j\rangle, \sigma}\left(c_{i, \sigma}^{\dagger} c_{j, \sigma}+c_{j, \sigma}^{\dagger} c_{i, \sigma}\right)+U \sum_{i} n_{i, \uparrow} n_{i, \downarrow}
$$

wobei i und j Gitterindizes bezeichnen und $\sigma=\uparrow, \downarrow$ den Spinzustand beschreibt. Die Summe über $\langle i, j\rangle$ berücksichtigt nur Wechselwirkungen mit dem nächsten Nachbarn, während $c_{i, \sigma}$ und $c_{j, \sigma}$ die fermionischen Vernichtungsund Erzeugungsoperatoren eines Elektrons mit beschriebenem Spin an der Gitterstelle $i$ entsprechend berücksichtigen. Der Operator $n_{i, \sigma}$ liefert die Gesamtzahl der Elektronen mit Spin, die an der Gitterstelle $i$ gefunden wurden [43]. Ob ein Material metallisches oder isolierendes Verhalten zeigt, hängt von den Beiträgen $t$ und $U$ ab.

In dem Fall, dass $U \ll t$ gilt (der einer schwachen Elektronenkorrelation entspricht), gewinnt ein Elektron die Energie $t$ und verliert im Gegenzug nur die Energie $U$, wenn es sich von Gitterstelle zu Gitterstelle bewegt.
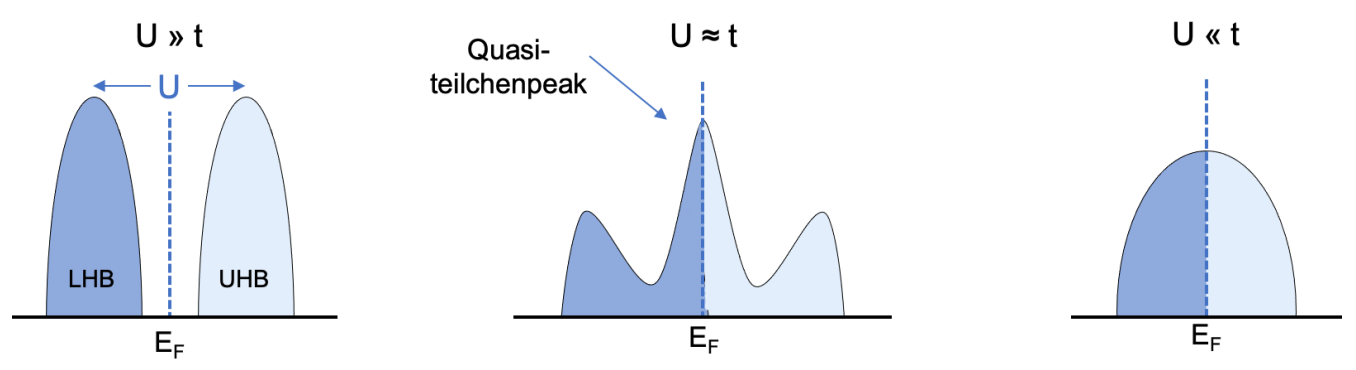

Abbildung 2.6.: Übergang vom isolierenden in den metallischen Zustand bei zunehmenden Verhältnis $U / t$ anhand des Hubbard-Modells und einhergehender Aufspaltung in zwei Hubbard-Bänder. Mit Erlaubnis nach dem Beispiel von [42] und [47]. 


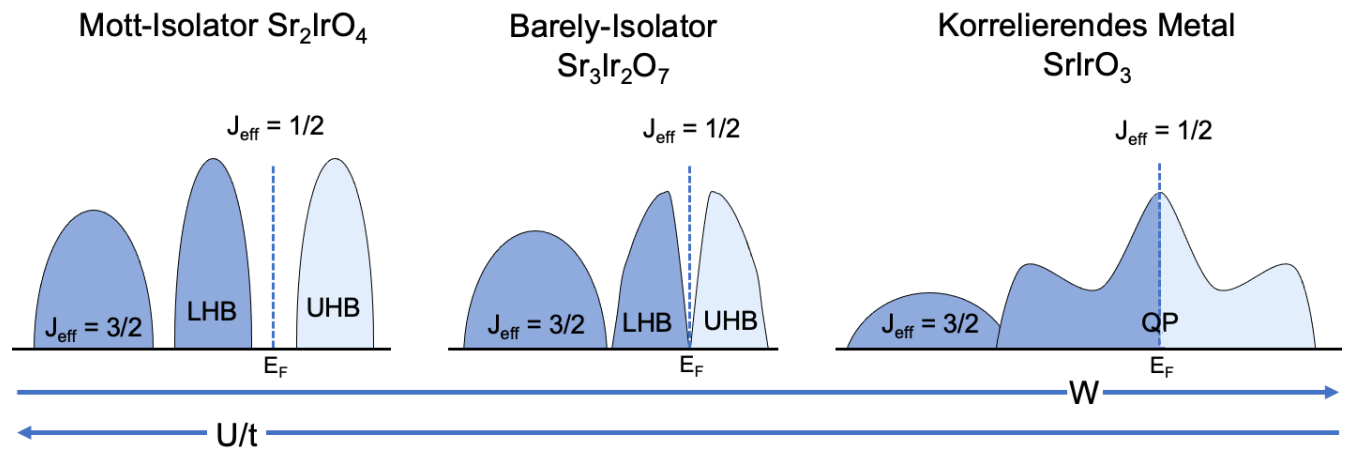

Abbildung 2.7.: Schematische Darstellung der Bandstruktur der 5d $\mathrm{Sr}_{n+1} \operatorname{Ir}_{n} \mathrm{O}_{3 n+1}$ Verbindungen mit $n=1,2$ und $\infty, E_{F}$ stellt das Fermi-Niveau dar und die blauen Pfeile illustrieren die Zunahme der Bandlücke in die eine Richtung und die $\mathrm{Zu}-$ nahme des Verhältnisses $U / t$ in die andere Richtung. Mit Erlaubnis nach dem Beispiel von [47].

Dadurch ist bei der Delokalisierung der Gewinn der Gesamtenergie größer, als die Coulombabstoßung der Elektronen, was zu einer metallischen Bandstruktur führt.

Überwiegt die Coulombabstoßung gegenüber der Hüpfamplitude $U \gg t$, so ist es energetisch günstiger jeden Platz mit nur einem Elektron zu besetzen. Hier ist der Gesamtenergiegewinn bei der Delokalisierung der Elektronen nicht groß genug, um die Coulombabstoßung zu überwinden. Die Elektronen sind lokalisiert und es stellt sich ein elektrisch isolierender Zustand ein, der als Mott-Hubbard-Isolator bezeichnet wird [32,43]. Hierbei müssen die Elektronen eine Energielücke überwinden, um den nächsten Nachbarn zu erreichen. Betrachtet man die Bandstruktur, so bilden sich zwei energetisch getrennte Subbänder, wie in Abbildung 2.6 gezeigt. Bei halber Bandfüllung und $T=0 \mathrm{~K}$ ist das untere Band, das sogenannte untere Hubbard-Band (LHB), vollständig belegt, während das obere Band (oberes Hubbard-Band (UHB)) leer ist, was den Mott-Isolationszustand definiert. Eine genauere Ableitung zur Bildung dieser Bänder ist in [44-46] nachzulesen.

Das Verhältnis zwischen $U$ und $t$ und somit die Frage nach der Leitfähigkeit, hängt stark mit der Bandbreite $W$ zusammen, da die Hüpfamplitude direkt proportional zur Bandbreite ist: $W \propto 2 Z t$ ( $Z$ der Ordnungszahl des Atoms). Die Entwicklung der Bandstruktur bei zunehmendem $W$ ist für 
die Ruddlesden-Popper-Verbindungen $\operatorname{Sr}_{n+1} \operatorname{Ir}_{n} \mathrm{O}_{3 n+1}$ mit $n=1,2$ und $\infty$ in Abbildung 2.7 dargestellt [47]. Für $\mathrm{SrIrO}_{3}$ ergibt sich der Fall, dass $U \approx t$ ist und das führt aufgrund der zunehmenden Korrelation zu verbreiterten Hubbard-Bändern und einem verengten Quasiteilchenpeak auf dem FermiNiveau, was ein anomales Metall darstellt.

Für die meisten $3 \mathrm{~d}$-Perowskite gilt hingegen $U \gg t$ (mit $U \approx 3-7 \mathrm{eV}$ und $\left.t_{0} \approx 0,1-0,3 \mathrm{eV}\right)$, weshalb im Grundzustand ein Mott-Isolator mit einem halb gefüllten Band beobachtet wird [10,48, 49].

\subsubsection{Austauschmechanismen}

In 3d-Perowskitoxiden ist der direkte Überlapp zwischen benachbarten BPlätzen vernachlässigbar gering und kann die Stärke der Austauschwechselwirkung nicht erklären. Vielmehr findet die Wechselwirkung über die Sauerstoffionen statt. Auf diese Weise treten zwei Wechselwirkungsmechanismen auf, die im Folgenden erklärt werden soll: der Erste wird als Superaustausch bezeichnet und beinhaltet den virtuellen Elektronenaustausch zwischen den Übergangsmetallkationen. Der Zweite ist der sogenannte Doppelaustausch, in dem die Austauschwechselwirkung über delokalisierte d-Elektronen und reelle Hüpfprozesse stattfindet.

Superaustausch Der Transfer eines Elektrons zwischen den B-Plätzen beinhaltet zwei Sprünge für jede B-O Bindung, wie in Abbildung 2.8 dargestellt. Das effektive d-d-Transferintegral (oder Hüpfamplitude) ist gegeben über $t_{d d}=t_{p d}^{2} / \Delta$, wobei $t_{p d}$ der einhergehende Energiegewinn bei der Delokalisierung der Elektronen zwischen den $\mathrm{O}-2 \mathrm{p}$ und B-3d-Zuständen ist und $\Delta$ die Energielücke des Ladungstransfers zwischen beiden Zuständen [10]. Aufgrund des Pauli-Prinzips ist der Hüpfprozess nur bei antiparalleler Ausrichtung der benachbarten Spins möglich und führt nach störungstheoretischen Rechnungen zweiter Ordnung zu einer Reduktion der Energie um:

$$
J_{S E}=-\frac{2 t_{d d}^{2}}{U}
$$

mit $U$ der bereits erwähnten Coulombenergie [10,32]. Bedingt durch die Geometrie der beteiligten Orbitale ist der Überlapp der O-2p mit den B- 
$t_{2 g}$ Orbitale doppelt so klein, wie der Überlapp zwischen den O- $2 p$ und $\mathrm{B}-e_{g}$ Orbitalen, wodurch die Austauschenergie $J_{S E}$ für die $e_{g}$-Zustände ungefähr 16-mal größer ist, als für die $t_{2 g}$-Zustände $[10,32]$. Aus diesem Grund genügt es, sich in der folgenden Betrachtung auf die Wechselwirkung zwischen den $\mathrm{O}-2 p$ - und den $\mathrm{B}-e_{g}$-Orbitalen zu konzentrieren. Zusätzlich hängt der Überlapp von dem B-O-B Bindungswinkel $\theta$ ab $(\propto \cos (\theta))$ und ist somit an die Verzerrung der Perowskit-Struktur gekoppelt [32].

Betrachtet man beispielsweise das $3 \mathrm{~d}$-Perowskit $\mathrm{LaMnO}_{3}\left(\mathrm{Mn}^{3+}\right)$ mit $U \approx$ $3,1 \mathrm{eV}$ und $t_{d d} \approx 0,4 \mathrm{eV}$, welches ohne Korrelation wegen seinem halb gefüllten $e_{g}$-Niveaus ein Metall wäre, aber aufgrund der Korrelation im Grundzustand ein Mott-Isolator ist [48]. Für $\mathrm{LaMnO}_{3}$ ist die Energielücke für den Ladungstransfer größer als die Hundenergie $(\Delta \approx 5 \mathrm{eV}>U)$, sodass das Loch beim virtuellen Hüpfen bevorzugt auf dem 3d-Ion entsteht [48]. Für andere Materialsysteme ist es jedoch auch möglich, dass das Loch bevorzugt auf dem Sauerstoffanion $(U \ll \Delta)$ entsteht. In diesem Fall spricht man von einem sogenannten Ladungstransfer-Isolator. In diesem Fall muss für $J_{S E}$ in Gleichung 2.1 das $1 / U$ durch $1 / \Delta$ ersetzen werden [10,32].

Die Art der magnetischen Kopplung (ob ferromagnetisch (FM) oder antiferromagnetisch (AFM)) ist letztlich auch von der Konfiguration der beteiligten Orbitale der Übergangsmetallionen im Kristallgitter abhängig und soll am Beispiel von $\mathrm{LaMnO}_{3}$ in Abbildung 2.8 illustriert werden. Aufgrund des Pauli-Prinzipes und der Hund'schen Kopplung ist das Hüpfen der Elektronen nur bei bestimmten Spinkonfigurationen erlaubt, die das Vorzeichen der Austauschwechselwirkung bestimmen. Wegen der starken Hund'schen Kopplung folgen die $e_{g}$-Elektronen der Orientierung der $t_{2 g^{-}}$ Elektronen [32]. Im $e_{g}^{1-} \mathrm{O}^{2-}-e_{g}^{1}$ System können sich somit die folgenden drei Fälle ergeben: a) das $e_{g}$-Elektron befindet sich bei beiden $\mathrm{Mn}^{3+}$-Kationen auf dem $d_{3 z^{2}-r^{2}}$-Orbital, wodurch sich eine antiferromagnetische Kopplung einstellt (dargestellt in Abbildung 2.8 a)), b) die beiden Elektronen besetzten statt der beiden $d_{3 z^{2}-r^{2}}$-Orbitale die $d_{x^{2}-y^{2}}$-Orbitale, wodurch sich ebenfalls eine AFM-Kopplung einstellt (Abbildung 2.8 b)), und schließlich c) ein Elektron befindet sich sowohl auf dem $d_{3 z^{2}-r^{2}}$ als auch auf dem $d_{x^{2}-y^{2}}$-Orbital, was in einer FM-Kopplung resultiert (Abbildung $2.8 \mathrm{c}$ )). 
Die magnetische Kopplung lässt sich mit Hilfe der sogenannten GoodenoughKanamori-Anderson-Regeln (GKA) [32,35,50-53] abschätzen und vorhersagen:

1. Der $180^{\circ}$-Austausch zwischen zwei leeren oder halb-gefüllten Orbitalen ist stark und antiferromagnetisch, entsprechend den Abbildungen 2.8 a) und b).

2. Der $180^{\circ}$-Austausch zwischen einem halb-gefüllten und einem leeren Oribital ist schwach und ferromagnetisch, Abbildung $2.8 \mathrm{c}$ ).

3. Der $90^{\circ}$-Austausch zwischen zwei halb-gefüllten Orbitalen ist schwach und ferromagnetisch. Dieser tritt jedoch nicht in der Perowskit-Struktur auf und wird daher nicht weiter betrachtet.
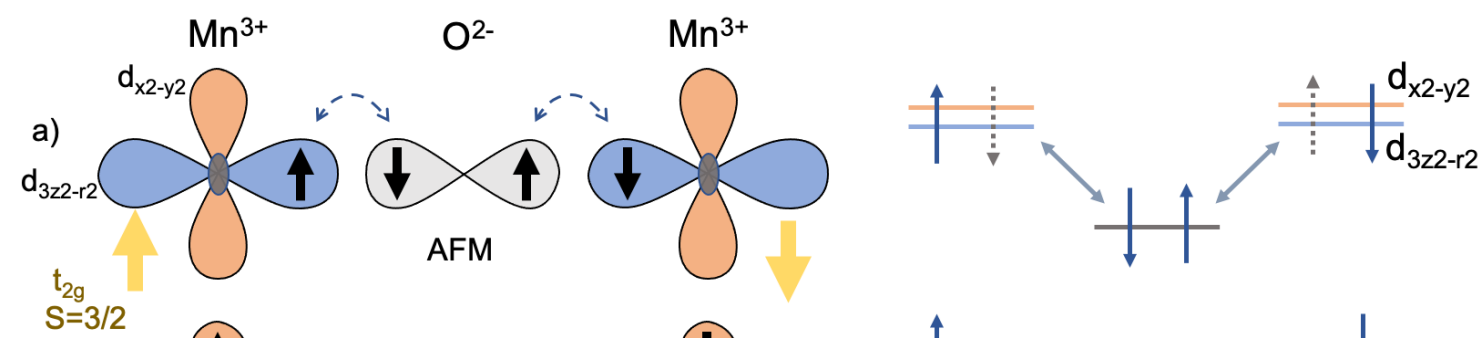

b)
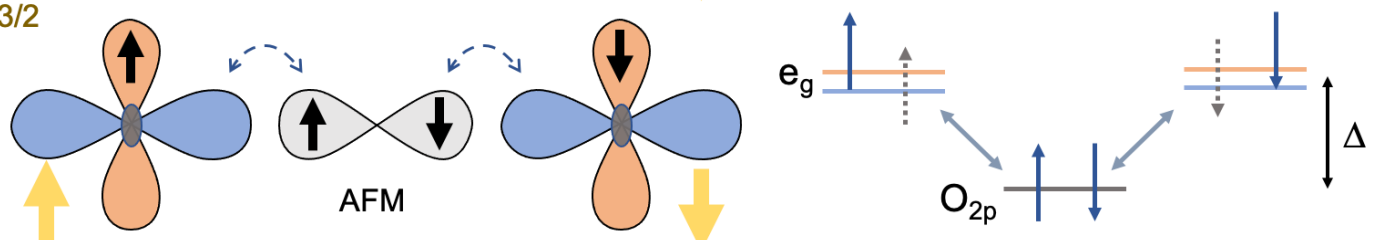

c)

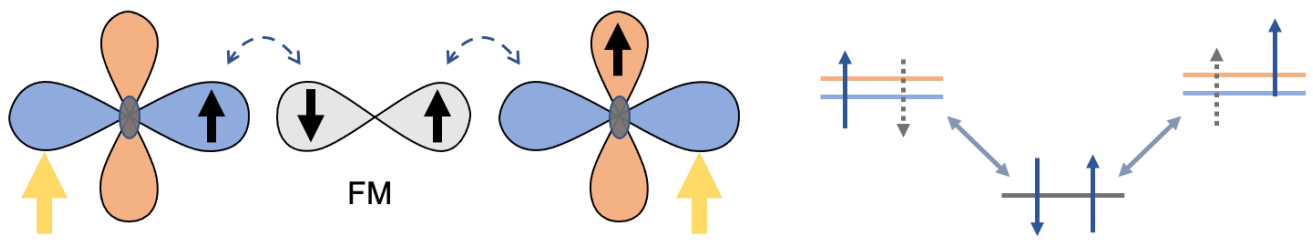

Abbildung 2.8.: Darstellung der magnetischen Austauschwechselwirkung zwischen zwei $\mathrm{Mn}^{3+}$ Kationen über das $2 p_{z}$-Orbital des Sauerstoffanions bei unterschiedlichen orbitalen Konfigurationen. Nach dem Beispiel von [32].

Doppelaustausch Im Gegensatz zum Superaustausch findet im Doppelaustausch ein reeller Hüpfprozess zwischen Übergangsmetallionen mit gemischter Valenz statt. Ein solches System lässt sich beispielsweise in $\mathrm{La}_{1-x} \mathrm{Sr}_{x} \mathrm{MnO}_{3}$ 
realisieren, in dem sowohl $\mathrm{Mn}^{3+}$ - als auch $\mathrm{Mn}^{4+}$-Kationen vorhanden sind, wie in Abbildung 2.9 dargestellt. Das $d_{3 z^{2}-r^{2}}$ Orbital des $\mathrm{Mn}^{3+}$ ist dabei einfach besetzt, während das des $\mathrm{Mn}^{4+}$ unbesetzt bleibt. So kann das $e_{g}-$ Elektron des $\mathrm{Mn}^{3+}$ über das $\mathrm{O}^{2-}$-Anion bei paralleler Spinausrichtung der dazugehörigen $t_{2 g}$-Elektronen ohne zusätzliche Aufbringung der Coulombenergie $U$ oder der Hund'schen Kopplung $J_{H}$ in das benachbarte leere $d_{3 z 2-r 2^{2}}$ Orbital des $\mathrm{Mn}^{4+}$-Kations hüpfen. Der Austausch wird hierbei über das gleichzeitige Hüpfen des $e_{g}$-Elektrons in das $2 p_{z}$-Orbital des Sauerstoffanions und des Elektrons im $2 p_{z}$-Orbital mit zum $e_{g}$-Elektron parallelem Spin in das leere $d_{3 z 2-r 2^{-}}$Orbital des $\mathrm{Mn}^{4+}$-Kations realisiert. Dies führt dazu, dass das 3d-Elektron im Gegensatz zum Superaustausch delokalisiert ist, sodass sich für die ferromagnetische Kopplung der gemischtvalenten Mangan-Ionen ein metallischer Zustand einstellt. Sind hingegen die Spinzustände der beteiligten Mn-Kationen antiparallel ausgerichtet, muss beim Transfer zusätzlich die Hund'sche Kopplungsenergie $J_{H}$ aufgebracht werden, die in der Regel größer ist als der Energiegewinn durch die Delokalisierung [32].

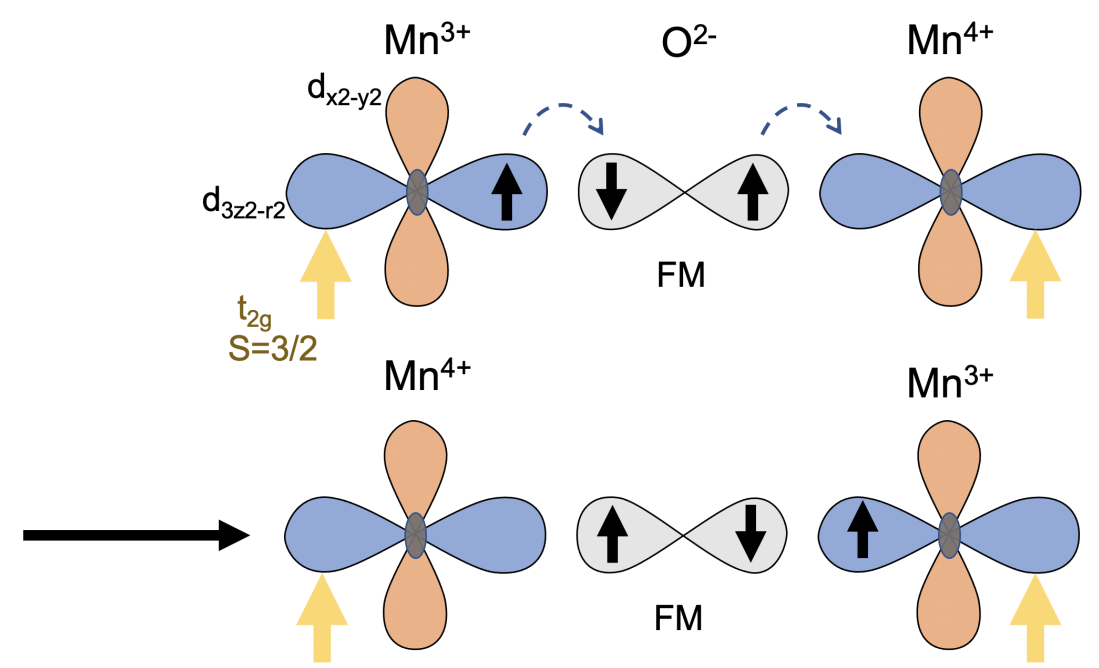

Abbildung 2.9.: Darstellung des Doppelaustausches des gemischvalenten Systemes mit einem $\mathrm{Mn}^{3+}$ und einem $\mathrm{Mn}^{4+}$ Kations über das Sauerstoffanion. Nach der Skizze aus [32]. 


\subsection{Ordnungsphänomene in Doppelperowskiten}

Im Gegensatz zu den einfachen Perowskitoxiden $\mathrm{ABO}_{3}$, sind in Doppelperowskiten (DP) die B-Plätze durch Kationen unterschiedlicher Art besetzt, was durch die unterschiedlichen Valenzen zu neuartigen Wechselwirkungen und Eigenschaften führen kann. Elektrische-, strukturelle- und magnetische Eigenschaften der DP hängen stark mit der B-Platzordnung zusammen. Diese Zusammenhänge werden in diesem Kapitel näher erläutert und deren Auswirkungen beschrieben.

\subsubsection{Kristallstruktur und Kationordnung}

Während in gewöhnlichen Perowskiten der B-Platz einfach besetzt ist, ist in Doppelperowskiten der B-Platz mit zwei unterschiedlichen Kationen B und B' im Verhältnis 1:1 besetzt. Verteilen sich die unterschiedlichen B-Kationen willkürlich im Kristall, gilt das Doppelperowskit als ungeordnet und die Notation $\mathrm{AB}_{0,5} \mathrm{~B}_{0,5}^{\prime} \mathrm{O}_{3}$ wird verwendet, während im geordneten Fall die BPlätze systematisch besetzt sind und man auf die Notation $\mathrm{A}_{2} \mathrm{BB}^{\prime} \mathrm{O}_{6}$ zurückgreift. Die B-Platzordnung des geordneten $\mathrm{A}_{2} \mathrm{BB}^{\prime} \mathrm{O}_{6}$ DP kann sich über drei Möglichkeiten einstellen: 1. der NaCl-Struktur, in der in alle drei Raumrichtungen abwechselnd B- und B'-Besetzung vorliegt (siehe Abbildung 2.10 a)), 2. die kolumnare Ordnung und 3. die lagenweise Ordnung [5,6]. Da im geordneten Fall in der Regel die beiden B-Kationen unterschiedlich geladen sind, ist aus elektrostatischer Sicht die NaCl-Struktur energetisch am günstigsten, da so die höher geladenen B-Kationen räumlich voneinander getrennt sind [5]. Deshalb tritt weitaus häufiger die NaCl-Struktur gegenüber den anderen beiden Ordnungsformen auf, sodass man sich im Folgenden ausschließlich auf die NaCl-Struktur konzentrieren wird.

Wie auch bei den einfachen Perowskiten hängt die Kristallstruktur des DP von der Größe der Kationen und somit vom Toleranzfaktor $t$ und der Verkippung der $\mathrm{BO}_{6} / \mathrm{B}^{\prime} \mathrm{O}_{6}$-Oktaeder ab. Im idealen Fall $t=1$ wird häufig die unverzerrte $F m \overline{3} m$-Struktur beobachtet, während für abweichende Toleranzfaktoren $t<1$ eine Kristallisation in der tetragonalen $I 4 / m$ - und in 
der monoklinen $P 2_{1} / n$-Raumgruppe beobachtet wird. Ungeordnete DP hingegen weisen oftmals eine orthorhombische Pnma-Struktur auf [6,54-59]. In der NaCl-Anordnung kann für $t=1$ neben der gewöhnlichen kubischen Einheitszelle eine erweiterte Einheitszelle betrachtet werden, die sich aus zwei kubischen Einheitszellen mit jeweils einem B- und B'- Kation zusammensetzt, mit $a_{D P}=2 a$ (daher die Bezeichnung Doppelperowskit). Dies wird deutlich, wenn man diejenigen Ebenen betrachtet, in denen sich auf den B/B'-Plätzen nur die gleichen Kationenarten befinden. So ergibt sich für die NaCl-Struktur entlang der (111)-Orientierung eine Überstruktur aus $\mathrm{ABO}_{3}$ - und $\mathrm{AB}^{\prime} \mathrm{O}_{3}$-Perowskiten [60] (siehe Abbildung $2.10 \mathrm{~b}$ )). Die verdoppelte Einheitszelle hat in geordneten DP in (111)-Orientierung zufolge, dass in Röntgendiffraktometrie Messungen ${ }^{1}$ (XRD, engl.: X-ray diffraction) neben dem (111)-Schichtpeak ein weiterer $(1 / 2,1 / 2,1 / 2)$-Peak erscheint, der der doppelten Einheitszelle zuzuordnen ist $[61,62]$.

Ob und inwiefern sich die Ordnung während des Wachstums einstellt hängt von unterschiedlichen treibenden Kräften ab. Wie auch schon für das Auftreten der NaCl-Struktur spielt die Elektrostatik und somit der Valenzunterschied der B-Kationen $\Delta Z_{B}=\left|Z_{B}-Z_{B^{\prime}}\right|$ eine entscheidende Rolle. Dabei gilt, je höher der Valenzunterschied, desto höher der Grad der Ordnung, da so der Abstand zwischen den höher geladenen B-Kationen maximiert wird $[5,6]$. Empirische Untersuchungen zeigen, dass Doppelperowskite mit einem Valenzunterschied von $\Delta Z_{B}>2$ in der Regel geordnet und mit $\Delta Z_{B}<2$ ungeordnet sind. Für $\Delta Z=2$ ergeben sich beide Fälle [6,59]. Des Weiteren spielt der Unterschied in den Kationenradien $\Delta R_{B}=\left|R_{B}-R_{B^{\prime}}\right|$ eine große Rolle bei Ausbildung der Ordnung, bei dem ebenfalls gilt: je größer der Unterschied, desto größer die Verspannungen und dadurch die Tendenz zur B-Platzordnung. Bemerkenswert ist an der Stelle, dass sich die beiden Faktoren $\Delta Z_{B}$ und $\Delta R_{B}$ gegenseitig beeinflussen und sogar verstärken können, da der Radius der jeweiligen Ionen von deren Valenz abhängt. Dies kann dazu führen, dass ein Valenzunterschied den Unterschied der Kationenradien weiter verstärkt, wodurch die B-Platzordnung weiter favorisiert werden kann. So beträgt der Größenunterschied der Kationenradien

\footnotetext{
${ }^{1}$ Zur Bestimmung der Kristallstruktur mittels Röntgenuntersuchung. Siehe Kapitel 4.
} 
a)

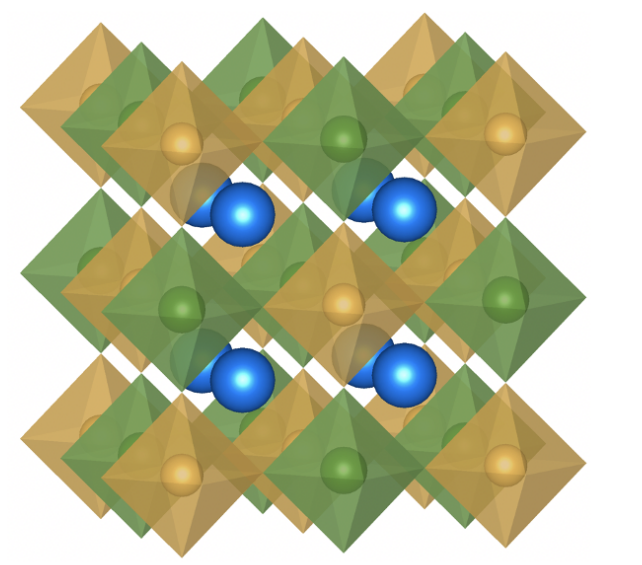

\section{A-Platz-Kation}

B-Platz-Kation

B'-Platz-Kation

Sauerstoff-Anion

b)

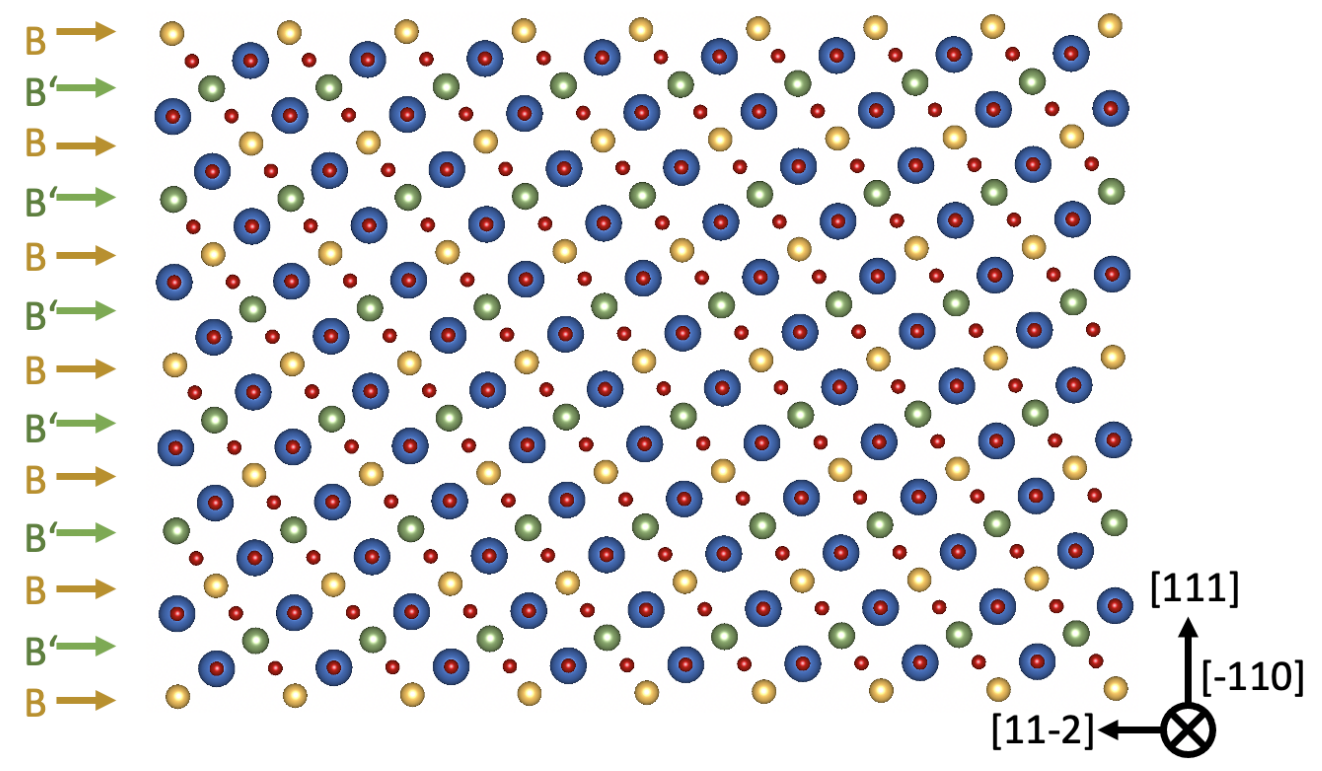

Abbildung 2.10.: a) Kristallstruktur eines geordneten Doppelperowskites in der NaCl-Struktur, b) Kristallordnung entlang der (111)Orientierung. Die alternierende $\mathrm{ABO}_{3}$ - und $\mathrm{AB}^{\prime} \mathrm{O}_{3}$-Lagen sind deutlich zu erkennen. Gezeichnet mit [28] und mit Erlaubnis angelehnt an [6].

für $\mathrm{La}_{2} \mathrm{CoMnO}_{6}$ im geordneten Fall $\left(\mathrm{Mn}^{4+} / \mathrm{Co}^{2+}\right.$, siehe nächster Abschnitt 2.2.2) $\Delta R_{B}=\left|R_{C o 2+}-R_{M n 4+}\right|=21 \mathrm{pm}$, während er im umgeordneten Fall lediglich $\Delta R_{B}=\left|R_{C o 3+}-R_{M n 3+}\right|=3 \mathrm{pm}$ erreicht [63]. Somit verstärkt die Ausbildung des Valenzunterschiedes den Unterschied in den Radien und somit die treibenden Kräfte zur Bildung der B-Platzordnung. Die Werte für die Ionenradien sind aus [64] entnommen. 
Neben den beiden genannten treibenden Kräften, die über die Wahl der Kationensorten festgelegt werden, kann die B-Platzordnung über die Depositionsbedingungen (Depositionstemperatur und -geschwindigkeit) der jeweiligen Wachstumsmethode und die Wahl des Substrates und somit der epitaktischen Verspannung beeinflusst werden [58,59,62].

Mittels der gängigen Wachstumsmethoden (PLD, MBE und Sputtering) wurden bereits zahlreiche Untersuchungen an Doppelperowskit-Dünnfilmen angestellt. Dabei stellen sich wesentliche Unterschiede innerhalb der jeweiligen Wachstumsmethode heraus, die starken Einfluss auf die DP und deren Ordnung haben. Da das zu deponierende Material (Atome, Cluster oder Moleküle) eine hohe kinetische Energie besitzt und so Defekte im Dünnfilm erzeugen kann, wird das Filmwachstum innerhalb der PLD und des Sputterns normalerweise als Nichtgleichgewichtsprozess angesehen. Die MBE hingegen ist frei von diesen Defekten und erreicht nahezu gleichgewichtige Wachstumsbedingungen. Jedoch arbeitet diese bei sehr geringem Sauerstoffpartialdruck, $p_{\mathrm{O}_{2}} \approx 10^{-5}$ mbar, was zu einem Sauerstoffdefizit im Film führen kann [65]. In Folge dessen führt dies dazu, dass die Proben nach der Herstellung an der Sauerstoffatmosphäre aufgeheizt werden müssen (im Folgenden als Post-Annealing bezeichnet), um nachträglich den Sauerstoffgehalt der Probe zu erhöhen [65]. Auch beim Sputtering und bei der PLD müssen für einen hohen Ordnungsgrad das Post-Annealing anwendent werden. Dies muss in Verbindung mit dem Nichtgleichgewichtszustand bei der Herstellung stehen, wodurch der Einbau möglicher Defekte die Ordnung verhindert [58,66-69].

Wachstumsbedingungen nahe dem Gleichgewichtszustand bei atmosphärischen Sauerstoffpartialdruck $\left(p_{\mathrm{O} 2}=0,2\right.$ bar $)$, wie bei den chemischen Methoden der MAD $[12,70]$ und PAD (abgekürzt für das englische polymerassisted deposition), [71] scheinen hingegen das Wachstum B-Platz geordneter Doppelperowskite zu begünstigen und kommen ohne anschließendes Post-Annealing aus. Das Wachstum der Dünnfilme resultiert im Fall der MAD aus einer sehr dichten Flüssig/Gas-Phase, die die Ausbildung großer Cluster und somit eines defektfreien Films begünstigt. 
Auch das A-Platzkation nimmt Einfluss auf das DP und beeinflusst über seine Valenz die durchschnittliche Valenz der B/B'-Kationen. So beträgt für ein $\mathrm{A}^{2+}$-Kation (beispielsweise $\mathrm{Ca}, \mathrm{Sr}, \mathrm{Ba}$ ) die durchschnittliche Valenz der B-Kationen vier und kann über folgende Valenzkombinationen realisiert werden: $\mathrm{B}^{4+} / \mathrm{B}^{\prime 4+}, \mathrm{B}^{3+} / \mathrm{B}^{15+}, \mathrm{B}^{2+} / \mathrm{B}^{\prime 6+}$ und schließlich $\mathrm{B}^{1+} / \mathrm{B}^{\prime 7+}$. Wo sich hingegen für ein $\mathrm{A}^{3+}$-Kation wie $\mathrm{La}, \mathrm{Pr}, \mathrm{Nd}$ usw. eine durchschnittliche Valenz von drei einstellt mit den Möglichkeiten: $\mathrm{B}^{3+} / \mathrm{B}^{\prime 3+}, \mathrm{B}^{2+} / \mathrm{B}^{\prime 4+}$ und $\mathrm{B}^{1+} / \mathrm{B}^{\prime 5+}[6]$. Welche Valenzverteilung sich letztlich einstellt, hängt neben der Ordnung auch von der Wahl der Elemente für die Kationen ab. Jedes Element hat individuelle Valenzzustände, die favorisiert werden.

Um eine Quantifizierung der B-Platzordnung treffen zu können, wird in der Literatur ein langreichweitiger Ordnungsparamter $S$ eingeführt:

$$
S=2 g_{B}-1
$$

wobei $g_{B}$ die Besetzung eines B/B'-Kations auf dem richtigen Gitterplatz bezeichnet $[6,61,63]$. Das vollständig geordnete Doppelperowskit entspricht einem Wert von $S=1$ und der des vollständig ungeordneten DP hingegen $S=0$. Zwischen den beiden Situationen weisen die Doppelperowskite eine Teil-Ordnung mit dem Ordnungsgrad $S$ auf. Abweichungen von der vollständig geordneten Phase können durch unterschiedliche Effekte auf-
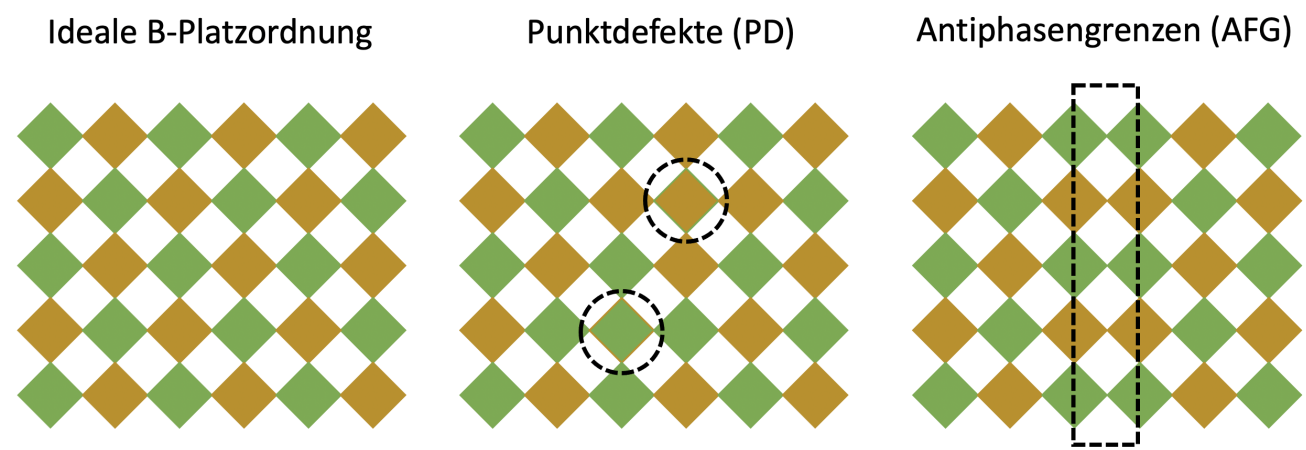

Abbildung 2.11.: Darstellung eines ideal geordneten Doppelperowskits in der NaCl-Struktur, Punktdefekten und Antiphasegrenzen. Die eingefärbten Quadrate stellen die $\mathrm{BO}_{6^{-}}$bzw. $\mathrm{B}^{\prime} \mathrm{O}_{6^{-}}$ Oktaeder dar. Mit Erlaubnis von Elsevier nach [6]. 
treten, die in Abbildung 2.11 dargestellt sind. Am häufigsten treten dabei Gitterfehlbesetzungen in Form von Punktdefekten (PD) auf, in denen die $\mathrm{B}$ - und $\mathrm{B}^{\prime}$-Kationen jeweils auf vertauschten Plätzen sitzen. Des Weiteren werden Antiphasengrenzen (APB, engl.: anti-phase boundary) beobachtet, die zwei geordnete Domänen mit umgekehrter B- und B'-Besetzung trennt, wie in Abbildung 2.11 rechts zu sehen [59]. Die jeweiligen Defekte wirken sich dabei unterschiedlich auf das DP aus. So beeinflussen die Punktdefekte nur lokal um den Besetzungsfehler das DP, während APB zu einer Reduzierung der langreichweitigen Ordnung führen können. Mittels Röntgendiffraktometrie lässt sich in geordneten DP in (111)-Orientierung durch das Intensitätsverhältnis des (111)- und (1/2 $1 / 2$ 1/2)-Peaks eine erste Abschätzung des Ordnungsparameters $S=I(1 / 21 / 21 / 2) / I(111)$ treffen $[61,72,73]$. Diese Abschätzung der B-Platzordnung mithilfe von XRD muss jedoch mit Vorsicht behandelt werden, da trotz hoher Ordnung aufgrund von Domänen mit entgegengesetzter Orientierung der Überstrukturpeak ausgelöscht sein kann, wodurch fehlerhaft ein zu geringer Wert für den Ordnungsparameter $S$ entsteht $[59,62]$. Auch der entgegensetzte Fall kann eintreten und eine Verstärkung des Überstrukturpeaks hervorrufen, in dem strukturelle Verzerrungen, Verkippungen der $\mathrm{B} / \mathrm{B}^{\prime} \mathrm{O}_{6}$-Oktaeder sowie Verschiebungen der A-Kationen, B-Kationen und $\mathrm{O}^{2-}$ Anionen innerhalb der Überstruktur der $\mathrm{NaCl-Anordnung} \mathrm{in} \mathrm{der} \mathrm{(111)-Richtung} \mathrm{eine} \mathrm{entsprechende} \mathrm{Abstandsmodu-}$ lation bilden. Dies kann so zu einer Nachahmung der Überstruktur in den Doppelperowskiten führen $[6,62,67,72,74]$, die sich in einem Überstrukturpeak widerspiegelt.

Es ist somit unerlässlich zur Festlegung der Ordnung weitere Parameter einzubeziehen. So können bei ferromagnetischen Proben Magnetisierungsmessungen Rückschlüsse auf die Ordnung im Kristall liefern. Darüber hinaus hat sich herausgestellt, dass die Raman-Spektroskopie aufgrund der unterschiedlichen Kristallstrukturen für geordnete und ungeordnete DP auch zur Bestimmung der B-Platzordnung geeignet ist. Es gelingt dieser Methode jedoch nicht, eine qualitative Aussage über den Grad der Kationordnung zu treffen. Letztlich können zur endgültigen Einschätzung Untersuchungen am Transmissionselektronenmikroskop (TEM, engl.: transmission elec- 
tron microscopy) in Verbindung mit Elektronenenergieverlustspektroskopie ${ }^{2}$ (EELS, engl.: electron energy loss spectroscopy) Messungen durchgeführt werden. Diese Messungen erlauben einen klaren Einblick in die lokale Ordnung des Kristalls unabhängig von der Orientierung gewachsener Domänen und Antiphasengrenzen.

Dies wird am Beispiel von geordnetem $\mathrm{La}_{2} \mathrm{CoMnO}_{6} / \mathrm{SrTiO}_{3}(111)$ deutlich, das mit der MAD gewachsen wurde [59]. Ein hoher Grad an Co/Mn-Ordnung im Nahbereich von $97 \%$ wurde auch durch die Magnetisierung mit hoher Sättigung bestätigt, $m_{S a t}=5,8 \mu_{B} /$ u.c. ${ }^{3}$. Die Valenzzustände der verantwortlichen Spins $\left(\mathrm{Mn}^{+2} / \mathrm{Co}^{+4}\right)$ wurden experimentell durch TEM- und EELS-Messungen bestimmt. Ermittelt man jedoch den Ordnungsparameter $S$ mittels XRD Messung, so fällt dieser deutlich geringer aus $S=1,1 \cdot 10^{-3}$, als der theoretisch vorhergesagte Wert $S_{\text {Theo }}=10^{-2}$ für ein vollständig geordnetes System. Diese Beobachtung wird voraussichtlich durch die erwähnten Domänen erzeugt, weshalb der Ordnungsgrad über die Überstrukturpeaks mit Vorsicht zu behandeln ist [59].

\footnotetext{
${ }^{2}$ Für Beschreibung zu TEM und EELS: siehe Kapitel 4.

${ }^{3} \mathrm{Im}$ geordneten Fall wird anhand der Spin-Konfiguration im System eine maximale Magnetisierung von $6 \mu_{B} /$ u.c. erwartet, während die Magnetisierung im ungeordneten Fall in einem Bereich von 3,5 bis 4,5 $\mu_{B}$ /u.c. liegt, wie im nächsten Abschnitt 2.2.2 näher erläutert wird.
} 


\subsubsection{Nickel und Kobalt basierte Doppelperowskite}

Die B-Platzordnung beeinflusst das Verhalten der Doppelperowskite maßgeblich. Dieses Phänomen soll im Folgenden anhand der beiden ferromagnetischen Isolatoren $\mathrm{La}_{2} \mathrm{NiMnO}_{6}$ und $\mathrm{La}_{2} \mathrm{CoMnO}_{6}$ dargestellt werden. Da die physikalischen Grundlagen und Eigenschaften der beiden DP ähnlich sind, soll zunächst das nickelbasierte Doppelperowskit näher beschrieben werden und die Erkenntnisse anschließend auf das DP $\mathrm{La}_{2} \mathrm{CoMnO}_{6}$ übertragen und auf die wesentlichen Unterschiede hingewiesen werden.

$\mathrm{La}_{2} \mathrm{NiMnO}_{6}$ Das Doppelperowskit hat aufgrund der ferromagnetischen Ordnung nahe der Raumtemperatur und der hochisolierenden elektrischen Eigenschaften $\rho(300 \mathrm{~K}) \approx 10^{2} \Omega \mathrm{cm}$, die beide $\mathrm{zu}$ einem relativ großen magnetodielektrischen Effekt führen $M D \approx 18 \%$, viel Aufmerksamkeit auf sich gezogen $[75,76]$. Aufgrund der trivalenten Valenz des Lanthan-Kations beträgt die durchschnittliche Valenz der $\mathrm{Mn} / \mathrm{Ni}-\mathrm{Kationen}$ drei. Wie sich die jeweilige Valenz auf die Ni/Mn-Kationen verteilt, hängt von der B-Platzordnung ab und hat weitreichenden Einfluss auf das Materialsystem. Im geordneten Fall erhält man in der $\mathrm{NaCl}$-Anordnung abwechselnd $\mathrm{Ni}^{2+}{ }_{-}$Kationen in der High-Spin-Konfiguration ([Ar] $] 3 \mathrm{~d}^{8}$ mit $\mathrm{t}_{2 g}^{6} \mathrm{e}_{g}^{2}, S=1$ ) und $\mathrm{Mn}^{4+}$ Kationen ebenfalls in der HS-Konfiguration ([Ar] $3 \mathrm{~d}^{3}$ mit $\mathrm{t}_{2 g}^{3} \mathrm{e}_{g}^{0}, S=\frac{3}{2}$ ), sodass jedes B-Kation in jede Richtung über ein Sauerstoffanion mit dem jeweilig anderem B-Kation verbunden ist. Diese Konfiguration führt nach der 2. GKA-Regel zu einem ferromagnetischen Superaustausch über das 2pOrbital des $\mathrm{O}^{-2}$-Anions mit einer maximalen Sättigungsmagnetisierung von $M_{s}=5 \mu_{B} /$ u.c. $\left(\left.\widehat{=} 2 \cdot \frac{3}{2}\right|_{S, M n}+\left.2 \cdot 1\right|_{S, N i}\right)$ und einer Curie-Temperatur von $T_{C} \approx 280 K[56,58,76,77]$. Durch Punktdefekte und Antiphasengrenzen entstehen jedoch $\mathrm{Mn}^{4+}-\mathrm{O}^{2-}-\mathrm{Mn}^{4+}$ und $\mathrm{Ni}^{2+}-\mathrm{O}^{2-}-\mathrm{Ni}^{2+}$ Konfigurationen, die nach der 1. GKA-Regel zu antiferromagnetischen Superaustauschwechselwirkungen führen und so eine Reduktion der Sättigungsmagnetisierung zufolge haben $[76,78]$. Da kein Doppelaustausch stattfindet, zeigt das Material isolierenden Charakter. Das geordnete DP kristallisiert in einer monoklinen Kristallstruktur mit der dazugehörigen Raumgruppe $P 12_{1} / n 1[56,77,79]$. Entlang der (111)-Orientierung lässt sich die Überstruktur der einfachen 
Perowskiten $\mathrm{LaNiO}_{3}$ und $\mathrm{LaMnO}_{3}$ beobachten.

Stellt sich hingegen eine willkürliche Ordnung der Ni/Mn-Kationen ein, erhält man ein $\mathrm{Ni}^{3+}-$ Kation mit Low-Spin-Konfiguration ([Ar] $3 \mathrm{~d}^{7} \operatorname{mit}_{2 g} \mathrm{t}_{g}^{6}$, $S=\frac{1}{2}$ ) und ein $\mathrm{Mn}^{3+}$-Kation mit High-Spin-Konfiguration ([Ar] $3 \mathrm{~d}^{4}$ mit $\left.\mathrm{t}_{2 g}^{3} \mathrm{e}_{g}^{1}, S=2\right)$, in dem ein $\mathrm{e}_{g}$-Elektron des Mn auf das Ni übergeht und über lokale Jahn-Teller-Verzerrungen stabilisiert wird [58,59,76]. Die daraus resultierenden $\mathrm{Ni}^{3+}-\mathrm{O}^{2-}-\mathrm{Ni}^{3+}, \mathrm{Ni}^{3+}-\mathrm{O}^{2-}-\mathrm{Mn}^{3+}$ als auch $\mathrm{Mn}^{3+}-\mathrm{O}^{2-}-\mathrm{Mn}^{3+}$ Kopplungen sind durch einen vibronischen Superaustausch (d.h. bei dem sich das Elektron mit gleicher Wahrscheinlichkeit auf beiden $\mathrm{e}_{g}$-Orbitalen befindet) nach der 2. GKA-Regel schwach ferromagnetisch. Die Unordnung der B-Kationen führt somit zu einer Reduzierung der Sättigungsmagnetisierung und der Curie-Temperatur $T_{C} \approx 130-140 K[58,76,78]$. Im Vergleich zur geordneten $P 12_{1} / n 1$-Struktur, zeigt das DP im ungeordneten Fall die orthorhombische Pbnm-Kristallstruktur [56,77,79]. Die Raman-Spektroskopie eignet sich aufgrund der unterschiedlichen Symmetrien und damit einhergehenden Auswahlregeln für die vibronischen Moden, um quantitativ die B-Platzordnung zu ermitteln $[58,59,76]$.

$\mathrm{La}_{2} \mathrm{CoMnO}_{6} \quad$ Mit wenigen Abweichungen sind die physikalischen Grundlagen des Doppelperowskites $\mathrm{La}_{2} \mathrm{CoMnO}_{6}$ dem des DP $\mathrm{La}_{2} \mathrm{NiMnO}_{6}$ sehr ähnlich. Auch hier befindet sich im geordneten Fall das $\mathrm{Co}^{2+}$-Kation in der HS-Konfiguration. Es besitzt jedoch ein Elektron weniger als das Nickel ([Ar] $3 \mathrm{~d}^{7}$ mit $\mathrm{t}_{2 g}^{5} \mathrm{e}_{g}^{2}, S=\frac{3}{2}$ ), sodass die Magnetisierung der Spin-Beiträge der ferromagnetischen $\mathrm{Co}^{2+}-\mathrm{O}^{2-}-\mathrm{Mn}^{4+}-$ Kopplung der B-Platz geordneten Phase maximal $M_{S}=6 \mu_{B} /$ u.c. beträgt mit einer Curie-Temperatur von $T_{C} \approx 230 K[73,78]$. Für eine ungeordnete B-Platzordnung stellt sich für das $\mathrm{Co}^{3+}$-Kation ein Intermediate-Spin Zustand ein ([Ar] $3 \mathrm{~d}^{6}$ mit $\mathrm{t}_{2 g}^{5} \mathrm{e}_{g}^{1}, S=1$ ), der ebenfalls zu einem vibronischen Superaustausch führt, der über JahnTeller-Verzerrungen stabilisiert wird. In der Literatur wird jedoch auch die Möglichkeit in Betracht gezogen, dass der vibronische Superaustausch durch einen Sauerstoffdefizit erzeugt wird [66,73]. Wie im $\mathrm{La}_{2} \mathrm{NiMnO}_{6}$ ist auch hier der vibronische Austausch weniger stabil und führt zu eine Reduzierung der Curie-Temperatur $T_{C} \approx 80-130 \mathrm{~K}$ und der Sättingungsmagnetisierung $M_{S} \approx 3,5-4,5 \mu_{B} /$ u.c. $[59,78]$. 


\subsection{Grenzflächenmagnetismus in oxidischen Heterostrukturen}

Es lassen sich eine Vielzahl oxidische Perowskite mit unterschiedlichen Aund B-Plätzen mit den gängigen Wachstumsmethoden epitaktisch aufeinander wachsen. An den Grenzflächen zwischen den jeweiligen Materialsystemen wechselwirken diese über ihre Freiheitsgrade des Gitters, der Ladung, der Orbitale und des Spins miteinander. Die jeweiligen Wechselwirkungen wirken sich dabei unterschiedlich auf das Materialsystem aus und können zu neuartigen magnetischen und elektrischen Effekten führen und eröffnen somit ein breites Feld der modernen Festkörperphysik. Der folgende Abschnitt soll einen Einblick in die gängigsten Mechanismen liefern, die in der Literatur diskutiert werden.

\subsubsection{Kopplungseffekte an Grenzflächen}

Ladungstransferbasierter Grenzflächenmagnetismus Ladungstransfer (CT, engl.: charge transfer) bezeichnet die Übertragung eines Elektrons von einem Materialsystem auf ein anderes an deren Grenzfläche. Wie auch an Grenzflächen von konventionellen Halbleitern und Metallen wird der CT in der Regel über den Unterschied im chemischen Potenzial der beiden Materialien begründet [10].

Es wird die Situation betrachtet, in der zwei Perowskite mit unterschiedlichem A-Kation und unterschiedlicher Valenz aufeinander gewachsen sind, während der B-Platz gleich bleibt. Diese Konstellation bewirkt, dass die nominelle Elektronenzahl auf dem Übergangsmetall über eine Grenzfläche variiert. Wie auch im einfachen Perowskit führt der Gewinn der kinetischen Energie zu einer Delokalisierung der Elektronen entlang der Grenzfläche. Der Energiegewinn wird auch hier über die Hüpfamplitude $t_{d d}$ charakterisiert und kann nur unter Aufbringung der Coulombenergie realisiert werden. Für die Ladungstrennung um die Gitterkonstante $a$ bei gegebener Permittivität $\epsilon$ des umgebenden Materials ist die Coulombenergie gegeben über $e^{2} / \epsilon a$, wobei $e$ die Elementarladung bezeichnet. Über die Hüpfamplitude und die Coulombenergie lässt sich in Analogie zur Thomas-Fermi-Abschirmlänge 
eine Längenskala für den Ladungstransfer angeben [10,80]:

$$
L_{T F} \approx \frac{t_{d d} \epsilon a^{2}}{e^{2}}
$$

Für typische oxidische Perowskite liegen die Werte für die Permittivität bei $\epsilon \approx 15$ und die Hüpfamplitude bei $t \approx 0,2 \mathrm{eV}$, womit sich eine Längenskala von 1-3 Monolagen für den Ladungstransfer ergibt [81]. Über die Delokalisierung der Elektronen stellt sich ein elektrostatisches Potenzial ein, das dem chemischen Potenzial der involvierten Materialien entgegenwirkt [10,82-87]. In einem Übergitter aus $\mathrm{LaMnO}_{3}$ und $\mathrm{SrMnO}_{3}$ stellt sich beispielsweise aufgrund der Valenz von $\mathrm{La}^{3+}$ und $\mathrm{Sr}^{2+}$ an der Grenzfläche ein abrupter Übergang von $\mathrm{Mn}^{3+}$ auf $\mathrm{Mn}^{4+}$ ein, der zu einem Ladungstransfer von der Spenderschicht $\mathrm{LaMnO}_{3}$ (unten) in die Akzeptorschicht $\mathrm{SrMnO}_{3}$ (oben) führt. Der CT verändert die Eigenschaften der Heterostruktur und führt im gegebenem Beispiel zu einer ferromagnetischen Phase mit einer hohen Curie Temperatur von $T_{C} \approx 360 \mathrm{~K}$. Mithilfe der in-situ-Ellipsometrie gelang es, die Längenskala des Ladungstransfers auf zwei Monolagen $\left(\lambda_{C T} \approx 2\right.$ u.c. $)$ direkt während des Wachstums mittels MAD zu bestimmen [12, 70,88].

Bringt man hingegen zwei Perowskite mit unterschiedlicher Besetzung der B-Plätze in Kontakt, lässt sich der Ladungstransfer beispielsweise durch Favorisierung bestimmter Valenzzustände in Analogie zu Redox-Reaktionen nur bedingt vorhersagen, sodass letztlich die Rückschlüsse aus dem Experiment gezogen werden müssen [10].

Ladungstransfer zwischen Perowskiten mit unterschiedlicher B-Besetzung findet beispielsweise an der Grenzfläche zwischen $\mathrm{LaNiO}_{3}$ und $\mathrm{LaMnO}_{3}$ von $\mathrm{Mn}$ auf $\mathrm{Ni}$ statt, $\mathrm{Mn}^{3+}+\mathrm{Ni}^{3+} \rightarrow \mathrm{Mn}^{4+}+\mathrm{Ni}^{2+}$. Zahlreiche Literaturquellen bestätigen diesen Effekt anhand von theoretischen Untersuchungen [84, 85] und Röntgenabsorptionsspektroskopie oder magnetischem Zirkulardichroismus (XMCD, engl.: X-ray magnetic circular dichroism) [82,86,87] und begründen diesen mit dem Unterschied des chemischen Potenziales zwischen den beiden Materialien. Neuste tiefgehende Untersuchungen am Materialsystem haben jedoch gezeigt, dass das Zusammenspiel der unterschiedlichen Grenzflächenmechanismen komplexer ist, als zu Beginn angenom- 
men. So hängt der Ladungstransfer sensibel von weiteren Mechanismen wie der struktureller Kopplung, dem Exchange Bias ${ }^{4}$, der Substratorientierung bis hin zu der Sequenz der Grenzfläche (LMO(top)/LNO(down) bzw. $\mathrm{LNO}\left(\right.$ top)/LMO(down)) ab. M. Gibert et al. [82] haben für auf $\mathrm{SrTiO}_{3}$ in (100)-Orientierung (STO(100)) gewachsenes $\mathrm{LaNiO}_{3} / \mathrm{LaMnO}_{3}$-Übergitter bewiesen, dass der magnetische Zustand des Mangan und das induzierte magnetische Moment in den Grenzflächen-LaNiO${ }_{3}$-Schichten signifikant von der Rauheit der Grenzflächen abhängen. Während scharfe Grenzflächen einen Kanten-Antiferromagnetismus in der $\mathrm{LaMnO}_{3}$-Schicht induzieren, führen die rauen Grenzflächen aufgrund des Ladungstransfers zu einer ferromagnetischen $\mathrm{Ni}^{2+}{ }_{-} \mathrm{Mn}^{4+}$-Austauschwechselwirkung an der Grenzfläche, ähnlich der der Wechselwirkung im Doppelperowskit $\mathrm{La}_{2} \mathrm{NiMnO}_{6}$ anhand der zweiten GKA-Regel [82]. Keine der vorherigen Studien berichtet jedoch über die Bildung und Eigenschaften des $\mathrm{La}_{2} \mathrm{NiMnO}_{6}$ an den Grenzflächen in diesen Heterostrukturen.

Entgegen der gängigen Beobachtungen wurde ein Ladungstransfer in Heterostrukturen aus $3 \mathrm{~d}-$ und 5d-TMO auch entgegen der chemischen Potenziale bzw. entgegen der Austrittsarbeit beobachtet [16-18]. Dieses Phänomen wird im Kapitel 2.3.2 gesondert betrachtet.

Strukturelle und orbitale Kopplung Die atomare Struktur und die magnetischen Eigenschaften sind über die Bindungslänge B-O und den Bindungswinkel B-O-B der B-Kationen direkt miteinander gekoppelt. Mithilfe von epitaktischem Wachstum auf Substraten mit unterschiedlichen Gitterkonstanten lassen sich kontrolliert Dünnfilme herstellen, deren Bindungslänge und Bindungswinkel kontrolliert modifiziert werden und sich somit die Eigenschaften von deren Bulkform unterscheiden. Wenn pseudokubische Perowskitfilme auf pseudokubischen Substraten mit Perowskitstruktur gewachsen werden, so passt sich die in-plane Gitterkonstante des gewachsenen Filmes der pseudokubischen Gitterkonstante des Substrates a an, was eine Kompression bzw. Dehnung der out-plane Gitterkonstanten $c$

\footnotetext{
${ }^{4}$ Der EB entsteht durch die Kopplung zwischen einem Ferromagnet und einem Antiferromagnet und sorgt für eine Verschiebung der magnetischen Hystereseschleife [89].
} 
des gewachsenen Filmes und damit einhergehenden Verzerrung der $\mathrm{BO}_{6}{ }^{-}$ Oktaeder zufolge hat. Wie bereits in Kapitel 2.1.1 gezeigt, hat dies weitreichenden Einfluss auf die Eigenschaften des Materialsystemes. Vergleichbar mit dem Jahn-Teller-Effekt hebt die Verzerrung die Entartung der $t_{2 g^{-}}$und $e_{g}$-Niveaus auf und beeinflusst so zusätzlich die orbitale Besetzung. Am Beispiel von $\mathrm{La}_{0.7} \mathrm{Sr}_{0.3} \mathrm{MnO}_{3}$ auf $\mathrm{LAO}(001)(c / a>1)$ und $\operatorname{STO}(100)(c / a<1)$ zeigt sich, dass eine Kompression durch das Substrat bevorzugt zu einer

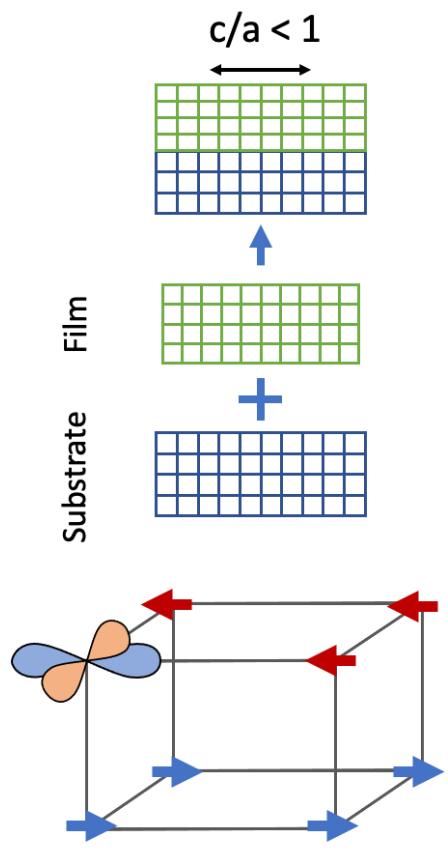

A-AFM $c / a=1$

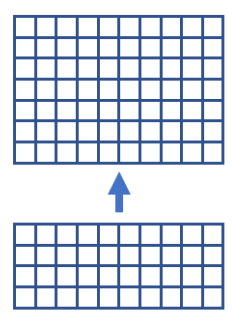

十
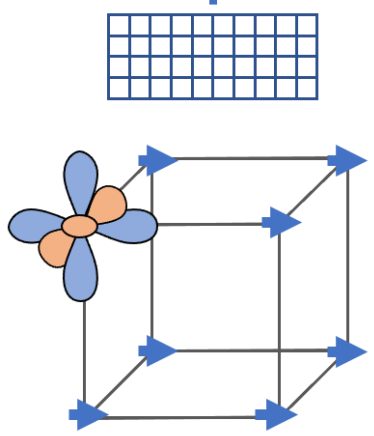

FM

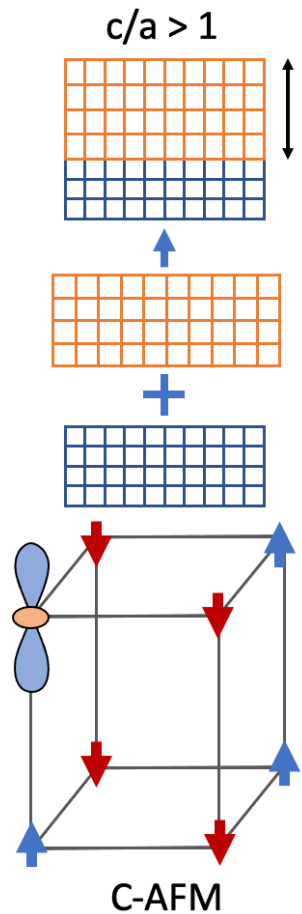

Abbildung 2.12.: Einfluss der epitaktischen Verspannung auf die orbitale Besetzung des $\mathrm{La}_{0.7} \mathrm{Sr}_{0.3} \mathrm{MnO}_{3}$ und daraus resultierende magnetische Ordnung. Links: Zugspannung $c / a<1$ induziert eine Besetzung der $d_{x^{2}-y^{2}}$-Orbitale und ermöglicht so eine FM-Wechselwirkung in der Ebene zwischen vollen und leeren $d_{x^{2}-y^{2}}$ Zuständen und AF-Wechselwirkungen außerhalb der Ebene zwischen vollen $t_{2 g}$-Zuständen, woraus ein Antiferromanetismus vom A-Typ resultiert. Mitte: Keine Verspannung, weshalb die $e_{g}$-Orbitale entartet bleiben. Rechts: Im Gegensatz dazu fördert bei Druckspannung $(c / a>1)$ eine Besetzung der $d_{3 z^{2}-r^{2} \text {-Orbitale eine }}$ FM-Wechselwirkungen außerhalb der Ebene und AFMWechselwirkungen innerhalb der Ebene, was zu einem AFM vom C-Typ führt. Mit Erlaubnis nach [90]. 
Besetzung der $d_{3 z^{2}-r^{2}}$-Orbitale führt und eine Dehnung zur Besetzung der $d_{x^{2}-y^{2}}$-Orbitale $[90,91]$. Dies führt wiederum zu unterschiedlichen magnetischen Ordnungen, wie in Abbildung $2.12 \mathrm{zu}$ sehen ist. Die strukturelle Kopplung zwischen dem Film und dem Substrat kann sich über mehrere zehn Einheitszellen ausbilden, bis diese relaxiert [10].

Zusätzlich zur veränderten Symmetrie des Gitters können auch Symmetriebrüche und Unterschiede in der oktaedrischen Rotation an Grenzflächen in Heterostrukturen zu einer bevorzugten Besetzung der Orbitale führen [92-94]. Um die Eckverbindung der $\mathrm{BO}_{6}$-Oktaeder über eine Grenzfläche aufrechtzuerhalten, werden die oktaedrischen Rotationen in der Nähe einer Grenzfläche modifiziert [10]. So werden beispielsweise Rotationen im grenznahen Bereich von orthorhombischen oder rhomboedrischen Perowskiten auf kubischen Perowskiten unterdrückt [95,96]. Im Gegensatz zu den Längenskalen der durch das Substrat entstehenden Verspannungen sind die Modifizierungen an der Grenzfläche deutlich kürzer ausgeprägt und befinden sich in der Größenordnung von ein bis zehn Einheitszellen [95-98].

Extrinsische Effekte: Stöchiometrie und Interdiffusion Die stetige Weiterentwicklung der gängigen Wachstumsmethoden, wie Molekularstrahlepitaxie, Sputtern und der gepulsten Laserdeposition aber auch die vakuumfreie Metallorganischen Aerosol Deposition, ermöglicht es Heterostrukturen von sehr hoher Qualität herzustellen. Dennoch lassen sich unerwünschte Effekte wie Sauerstoffleerstellen, lokale stöchiometrische Abweichungen oder atomare Diffusion an Grenzflächen nie vollständig vermeiden. Diese Effekte können einen erheblichen Einfluss auf die magnetische Struktur des Materials und der Grenzfläche haben und müssen bei der Interpretation der wissenschaftlichen Ergebnisse immer mitberücksichtigt werden.

Insbesondere bei den Wachstumsmethoden unter Vakuumbedingegungen sind Sauerstoffleerstellen oft nicht zu verhindern [65]. Die Sauerstoffleerstellen koppeln mit dem Gitter und können die B-O Bindungslänge und somit die Kristallstruktur über zusätzliche oktaedrische Verzerrungen und Rotationen verändern. Darüber hinaus kann die Valenz der B-Kationen reduziert werden, was sich auf die magnetischen Wechselwirkungen auswirkt. Besonders deutlich wird der Einfluss der Sauerstoffleerstellen bei $\mathrm{LaMnO}_{3}$, 
das in seiner stöchiometrischen Bulkform ein A-Typ Antiferromagnet ist, als Dünnfilm aufgrund der Sauerstoffleerstellen jedoch ferromagnetische Eigenschaften zeigt $[49,99]$. Wie bereits erwähnt, kann mit Hilfe von PostAnnealing an der Sauerstoffatmosphäre die Sauerstoffleerstellen weitestgehend beseitigt werden [63,65]. Die in dieser Arbeit hergestellten Proben sind allesamt mittels MAD hergestellt, die unter Atmosphärendruck arbeitet, wodurch der Einbau von Sauerstoffleerstellen während des Wachstums unwahrscheinlich ist.

Darüber hinaus können stöchiometrische Veränderungen zwischen den $\mathrm{A} / \mathrm{A}^{\prime}$ und $\mathrm{B} / \mathrm{B}^{\prime}-$ Kationen im Bereich der Grenzfläche durch Diffusion während des Wachstums entstehen. Am Beispiel von $\mathrm{La}_{1-x} \mathrm{Sr}_{x} \mathrm{MnO}_{3}$ wird dies aufgrund seines reichen Phasendiagrammes deutlich. Es reagiert empfindlich auf stöchiometrische Änderungen, was sich in dem Beispiel auf Grenzflächen zwischen $\mathrm{LaMnO}_{3} / \mathrm{SrMnO}_{3}$ auswirken kann. Durch Erniedrigung der Depositionstemperatur lässt sich die Diffusion unterdrücken, allerdings kann dies wiederum Einfluss auf die Wachstumskinetik haben und die Oberflächenmorphologie verändern.

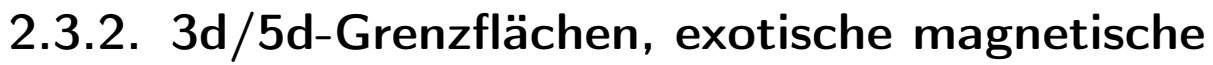 Phasen (Skyrmionen)}

In Kapitel 2.1.1 wurde bereits die Bedeutung der Spin-Bahn-Kopplung in 5d-TMO hervorgehoben. Entsprechend wirkt sich die Spin-Bahn-Kopplung auch auf Grenzflächen zwischen 3d- und 5d-TMO aus und ermöglicht so die Realisierung neuartiger Grenzflächeneffekte. Gegenstand aktueller Forschung ist dabei die Suche nach Grenzflächen, die den sogenannten topologischen Halleffekt (THE) aufweisen, der mit der Bildung von sogenannten Skyrmionen in Verbindung gebracht wird [19-24]. Skyrmionen sind nanowirbelartige topologische Spindefekte im Realraum, die in einer ferromagnetischen Umgebung auch bei kleinen Größen eine hohe Stabilität aufweisen. Deshalb gelten sie als vielversprechende Kandidaten für zukünftige Informationsträger und Logikelemente, was ihnen große Aufmerksamkeit einbringt [25]. Der THE konnte bereits beobachtet werden. So beispielsweise in Bilayern und Heterostrukturen aus $\mathrm{SrRuO}_{3}$ und $\mathrm{SrIrO}_{3}$ [14], in Bilay- 
ern aus $\mathrm{La}_{0.7} \mathrm{Sr}_{0.3} \mathrm{MnO}_{3}$ und $\mathrm{SrIrO}_{3}$ [21] und so wie in Heterostrukturen aus $\mathrm{LaMnO}_{3}$ und $\mathrm{SrIrO}_{3}$ mit asymmetrischen Grenzflächen [20]. In Heterostrukturen aus $\mathrm{SrMnO}_{3}$ aus $\mathrm{SrIrO}_{3}$ wurde hingegen kein THE beobachtet, wohl jedoch der sogenannte anomale Halleffekt (AHE) [16], der im kommenden Abschnitt genauer erklärt wird. Die individuellen Unterschiede der vorgestellten Materialsysteme zeigen, dass sich das Auftreten des THE und die damit verbundenen Grenzflächeneffekte nur schwer vorhersagen lassen und durch das Experiment überprüft werden müssen. Um die Physik der individuell auftretenden Effekte nachvollziehen zu können, sollen im Folgenden die Grundlagen für das Auftreten des anomalen und topologischen Halleffektes beschrieben werden.

Anomaler und topologischer Halleffekt Im klassischen Halleffekt (normaler Halleffekt) führt ein extern angelegtes Magnetfeld zu einem elektrischen Feld innerhalb der Probe, das sich senkrecht zum angelegten Strom und Magnetfeld aufbaut. Dieser Effekt resultiert aus der Lorentzkraft auf die Elektronen, die in ihrer Laufbahn zu einer Kante der Probe hin abgelenkt werden. Dies wirkt sich auf den transversalen spezifischen Widerstand $\rho_{x, y}$ aus, der nach dem Drude-Modell proportional zum Feld $B$ ist.

Daneben können in ferromagnetischen Systemen unter bestimmten Voraussetzungen auch der anomale und topologische Halleffekt beobachtet werden, die sich unterschiedlich auf die Messung des spezifischen Widerstandes auswirken, wie in Abbildung $2.13 \mathrm{zu}$ sehen ist. Der anomale Halleffekt ist dabei ein zusätzlicher Beitrag in ferromagnetischen Systemen zum spezifischen Widerstand $\rho_{x y}$, der proportional zur Magnetisierung $M$ des Systems ist [100] und auf den normalen Halleffekt aufaddiert wird:

$$
\rho_{x y}=\rho_{N H E}+\rho_{A H E}=R_{H}(T) \cdot B+R_{A H E}(T) \cdot \mu_{0} M(B) .
$$

$\rho_{N H E}$ und $\rho_{A H E}$ sind jeweils die spezifischen Widerstände des normalen und anomalen Halleffektes, $R_{H, A H E}$ die verschiedenen Koeffizienten der HallBeiträge, $\mu_{0}$ das Bohrsche Magneton und $M(B)$ die Magnetisierung der Probe. Der anomale Halleffekt ist auf die Spin-Bahn-Kopplung zurückzuführen, die zu zwei extrinsischen Streumechanismen führt, die unterschiedlich 
bevorzugte Streurichtungen für Spin-Up- und -Down-Teilchen haben [100]. Als Ergebnis dieser Streuung werden die ankommenden Teilchen mit jeweiligem Spin zu einer Kante der Probe gestreut. In einem Ferromagneten sind die Spin-Teilbänder ungleich besetzt, sodass dieses Ungleichgewicht zu einer Ladungsakkumulation an gegenüberliegenden Kanten führt, wodurch ein transversales elektrisches Feld erzeugt wird. Der anomale Beitrag $\rho_{A H E}$ liefert einen nicht linearen Beitrag zum spezifischen Widerstand, der abhängig von der Magnetisierung ist (in Abbildung 2.13 dargestellt). Bei den zwei extrinsischen Streumechanismen wird zwischen einem spinabhängigen Beitrag (engl.: Skew-Scattering) [101,102] und einem Beitrag, der durch einen lateralen Shift bei der Streuung entsteht (engl.: Side-Jump) [103,104], unter-
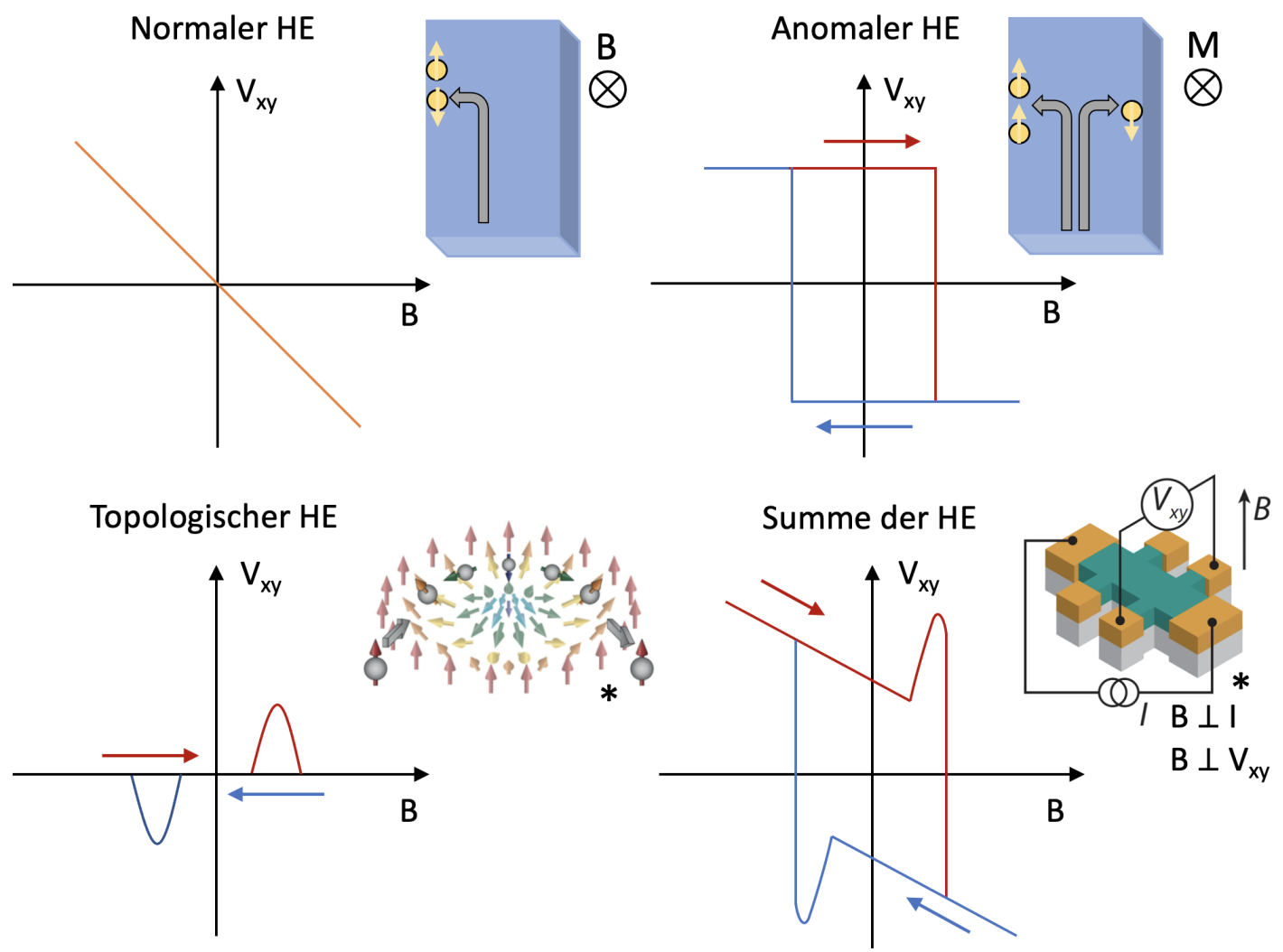

Abbildung 2.13.: Schematische Darstellung des Einflusses des normalen, anomalen und des topologischen Halleffektes auf die Messung des transversalen spezifischen Widerstandes in Abhängigkeit vom angelegten Magnetfeld. Mit Erlaubnis nach den Beispielen aus [26]. Mit * markierte Bilder sind entnommen aus [23]. 
schieden. Zusätzlich zu diesen extrinsischen Beiträgen, wird ein intrinsischer Beitrag beobachtet, der über den nicht trivialen Begriff der Berry-Phase beschrieben wird, in denen der Spin eines Elektrons der räumlichen variierenden Magnetisierung folgt [105-107]. Auf die umfangreiche theoretische Beschreibung der einzelnen Beiträge wird an dieser Stelle verzichtet. Sie kann in [100-107] nachvollzogen werden.

Der topologische Halleffekt entsteht, wenn ein Elektron eine variierende Magnetisierungstextur durchläuft. Der Ursprung dafür liegt meist in der sogenannten Dzyaloshinskii- Moriya-Wechselwirkung (DMI). Bei einer gebrochenen Inversionssymmetrie kann es bei Anwesenheit der Spin-Bahn-Kopplung zu einer Wechselwirkung benachbarter Spins kommen, die zu einer Neigung zwischen diesen führt. Beschrieben wird dieser Effekt durch einen Energiekorrekturterm zwischen den benachbarten Spins $S_{i, j}$. Dieser ist gegeben über [107-109]:

$$
H_{D M I}=\frac{1}{2} \sum_{i, j} \boldsymbol{D}_{i, j} \cdot\left(S_{i} \times S_{j}\right)
$$

wobei $\boldsymbol{D}_{i, j}$ den Dzyaloshinskii-Moriya-Vektor darstellt, der die Wechselwirkung den beiden Spins beschreibt. Das Kreuzprodukt der Gleichung zeigt, dass die Wechselwirkung die Spins aus ihrer kollinearen Anordnung heraus verkippt, um ihre Energie zu minimieren, woraus schließlich die Skyrmionen entstehen. Die beteiligten Spins drehen sich dabei kontinuierlich von innen nach außen, sodass sie am Rand und in der Mitte des Skymions entgegengesetzt sind. Aus dieser Wechselwirkung heraus können Skyrmionen in den unterschiedlichsten topologischen Formen entstehen [19,22].

Sofern Skymionen vorhanden sind, entsteht neben dem normalen und dem anomalen Halleffekt ein weiterer Beitrag zum spezifischen Widerstand. Der topologische Halleffekt ist proportional zu der Skyrmionendichte $n_{S k y}$ [19] und wird über das Konzept der Berry-Phase im Realraum beschrieben. In Abbildung 2.13 ist der Einfluss des topologischen Halleffektes auf die Messung der spezifischen Widerstandes schematisch dargestellt. 
$\mathrm{SrMnO}_{3} / \mathrm{SrlrO}_{3}$-Heterostrukturen Im Rahmen der vorliegenden Arbeit werden Heterostrukturen aus 3d- und 5d-TMO untersucht, die sich aus dem 3d-System $\mathrm{SrMnO}_{3}$ (mit schwacher Spin-Bahn-Kopplung $\xi=0,01-0,1 \mathrm{eV}$ und starker Korrelation $U=5-7 \mathrm{eV}$ ) und dem 5d-System $\mathrm{SrIrO}_{3}$ (mit starker SOC $\xi=1-3 \mathrm{eV}$ und moderater Korrelation $U=1-3 \mathrm{eV}$ ) zusammensetzt [110]. Eine solche Probengeometrie ermöglicht die Untersuchung von 3d/5d-Grenzflächen, bei denen sowohl $U$ als auch $\xi$ zusammen stärker sind als in beiden Ausgangsverbindungen $[16,17]$. Erste Untersuchungen an dem vorliegenden System wurden von den Gruppen um Okamoto und Nichols durchgeführt, deren Ergebnisse zusammengefasst vorgestellt werden sollen.

Die untersuchten Proben $\left(\mathrm{SrIrO}_{3}\right)_{n} /\left(\mathrm{SrMnO}_{3}\right)_{n}$ mit $n=1,2, \ldots 6$ wurden auf STO-Substraten in (100)-Orientierung mittels PLD hergestellt. Für $n \leq 3$ deuten die Magnetisierungsmessungen auf die Ausbildung einer ferromagnetischen Phase an der Grenzfläche hin, die sich sowohl im $\mathrm{SrMnO}_{3}$ als auch im $\mathrm{SrIrO}_{3}$ einstellt. Je kleiner $n$, desto stärker prägt sich diese Phase aus. Für $n>3$ wird keine ferromagnetische Phase beobachtet. Grundlage für die Ausbildung der ferromagnetischen Grenzflächenkopplung ist ein Ladungstransfer vom Iridium in das Mangan, der mittels Röntgenabsorptionsspek-

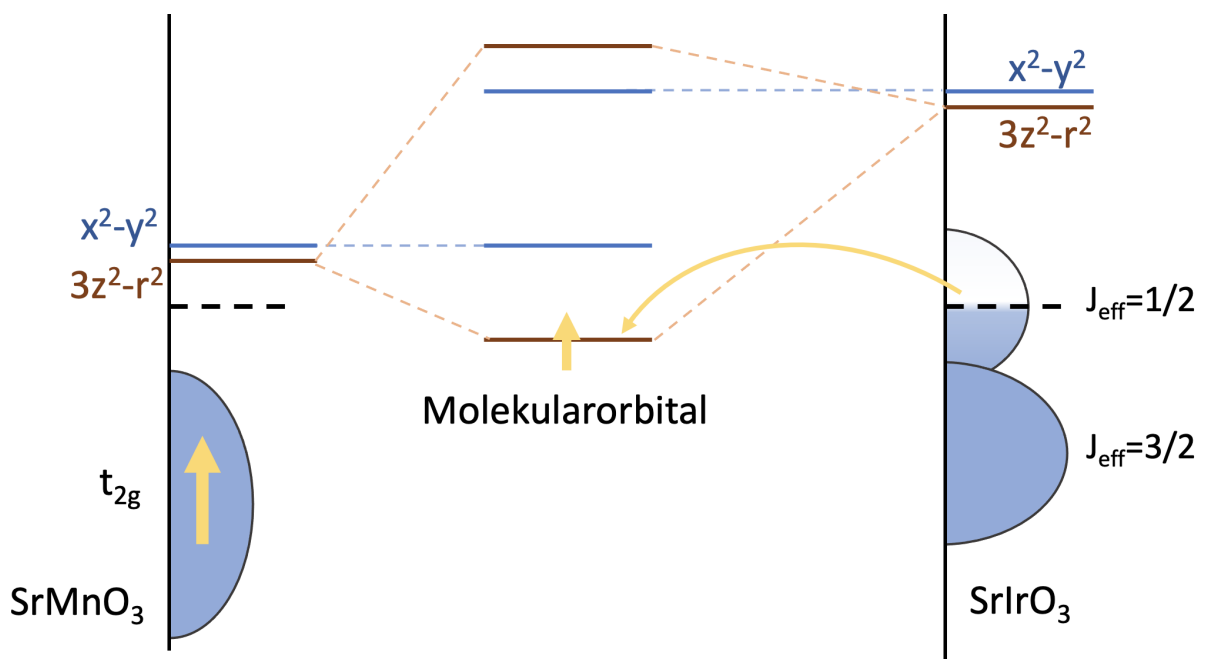

Abbildung 2.14.: Schematische Dichte der Zustände von $\mathrm{SrMnO}_{3}$ und $\mathrm{SrIrO}_{3}$ und das an der Grenzfläche zwischen ihnen gebildete Molekülorbital. Nach dem Schema aus [17]. 
troskopie (XAS) und röntgenmagnetischen Zirkulardichroismus (XMCD) beobachtet wird. Aufgrund der nahezu identischen Austrittsarbeit zwischen $\mathrm{SrIrO}_{3}$ und $\mathrm{SrMnO}_{3}, 5,05 \mathrm{eV}$ bzw. 4,99 eV [111] würde man intuitiv jedoch erwarten, dass die Menge der übertragenen Elektronen ziemlich gering ist und sogar in die entgegengesetzte Richtung von $\mathrm{SrMnO}_{3}$ zu $\mathrm{SrIrO}_{3}$ verläuft. Dass dennoch ein $\mathrm{CT}$ vom $\mathrm{SrIrO}_{3}$ zum $\mathrm{SrMnO}_{3}$ stattfindet, wird mit dem molekularen Orbitalmodel auf Basis der Untersuchungen von Okamoto [112] begründet. Es wird mit DFT-Rechnungen zusammen mit XASund Ellipsometriemessungen [17] bestätigt. Dabei handelt es sich um einen phänomenologischen Ansatz, der eine physikalische Anschauung des Sachverhaltes bietet und in Abbildung 2.14 veranschaulicht wird.

$\mathrm{SrMnO}_{3}$ hat die $t_{2 g}^{3}$-Konfiguration mit $S=3 / 2$ und ein leeres, zweifach entartetes $e_{g}$-Orbital $\left(x^{2}-y^{2}\right.$ und $\left.3 z^{2}-r^{2}\right)$, während sich das $\mathrm{SrIrO}_{3}$, wie in Kapitel 2.1.1 beschreiben, in der $t_{2 g}^{5}$-Konfiguration befindet, das aufgrund der starken Spin-Bahn-Kopplung weiter aufgeteilt wird in ein einfach besetztes Niveau mit Gesamtdrehimpuls $J_{\text {eff }}=1 / 2$ und ein vierfach besetztes Niveau mit $J_{\text {eff }}=3 / 2$. Das $e_{g}$-Niveau bleibt leer und befindet sich weit über dem Fermi-Niveau. Betrachtet man nun die Elektronenkonfiguration beider Ausgangspositionen und ihre ähnlichen Austrittsarbeiten, so entsteht aufgrund des großen Überlappens der $3 z^{2}-r^{2}$-Orbitale der benachbarten Mnund Ir-Atome ein sogenanntes Molekülorbital (siehe Abbildung 2.14). Das antibindene Molekülorbital liegt über dem $3 z^{2}-r^{2}$-Orbital des Ir, während das bindende Molekülorbital unter dem Mn-Orbital liegt. Die Bildung von Molekülorbitalen für das $x^{2}-y^{2}$ bleibt hingegen aufgrund der schwächeren Hybridisierung entlang der z-Richtung aus. Letztlich ergibt sich so ein $3 z^{2}-r^{2}$-Orbital, das unter der dem Fermi-Niveau liegt, weshalb Elektronen aus dem $J_{\text {eff }}=1 / 2$ Niveau in das bindende $3 z^{2}-r^{2}$-Orbital fallen. Dieser Vorgang entspricht schlussendlich einem Ladungstransfer vom $\mathrm{SrIrO}_{3}$ in das $\mathrm{SrMnO}_{3}[17]$.

Aufgrund der ferromagnetischen Phase zeigt das System für $n \leq 3$ den anomalen Halleffekt, wohingegen keine Berichte über das Auftreten des topologischen Halleffektes vorliegen. 



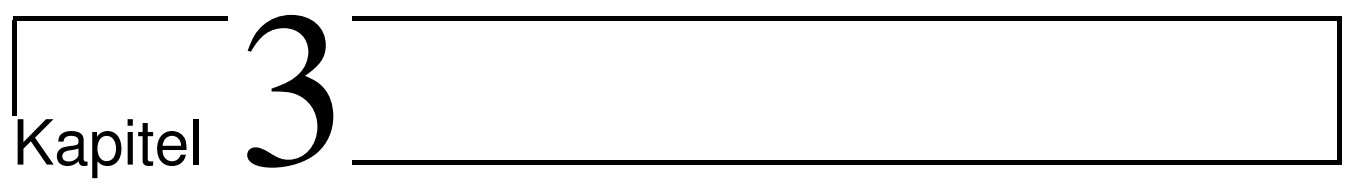

\section{Metallorganische Aerosol Deposition}

\section{(MAD)}

Die Metallorganische Aerosol Deposition (MAD, engl.: metal organic aerosol deposition) ist eine chemische Methode, die die Herstellung hoch qualitativer oxidischer Dünnfilme ermöglicht $[12,59,70,113]$ und mit dessen Hilfe sämtliche Proben hergestellt wurden, die in dieser Arbeit vorgestellt werden. Verglichen mit den konventionellen Vakuumdepositionsmethoden PLD, Sputtern und MBE zeichnet sich die MAD durch ihre vakuumfreien Wachstumsbedingungen aus, die eine Reihe von Vorteilen aufweisen. Neben des vergleichsweise einfachen und schnellen Herstellen von Probensystemen, gewährleistet der vorhandene Sauerstoffpartialdruck $\left(p_{\mathrm{O}_{2}}=0,2 \mathrm{bar}\right)$ das Ausbleiben von Sauerstoffleerstellen. Gleichzeitig schließt der vakuumfreie Betrieb jedoch die gängige in-situ-Wachstumskontrollen RHEED für die Nutzung an der MAD aus, wie sie in den konventionellen Depositionsmethoden üblich ist. Aus diesem Grund wurde die MAD-Anlage zusätzlich mit der optischen Ellipsometriemessung ausgestattet, die mit Hilfe der Änderung der Polarisation von Licht nach der Reflexion an der zu untersuchten Probe Aussagen über das Materialsystem ermöglicht.

Das vorliegende Kapitel soll das Prinzip der MAD und der Ellipsometrie vorstellen. Dabei wird zunächst das Grundkonzept der MAD vorgestellt, bevor auf die theoretischen Grundlagen und die technische Realisierung der in-situ-Ellipsometrie eingegangen wird. 


\subsection{MAD Grundlagen und Aufbau}

Abbildung 3.1 zeigt den schematischen Aufbau der verwendeten MADAnlage. Ausgangspunkt zur Herstellung der Dünnfilme bilden dabei metallorganische Präkusoren (üblicherweise Acetylacetonat-Komplexe), die im gewünschten molaren Verhältnis und Konzentration in Dimethylformamid (DMF), einem organischen polaren Lösungsmittel, gelöst werden. Das Herzstück der Anlage bildet die pneumatische Düse, die mittels Druckluft ( 5 bar) die Lösung in ein feines Aerosol zerstäubt und auf ein Substrat richtet. Das Substrat befindet sich im Zentrum des Aerosolkegels auf einem SiC-Heizer, der in Abhängigkeit des zu wachsenden Materialsystemes die Oberfläche des Substrates in einem Bereich von $T_{D e p}=600^{\circ}-950^{\circ}$ erhitzt. Durch die erhöhte Temperatur kommt es unmittelbar über dem erhitzen Substrat zur sogenannten Pyrolysereaktion, bei der die Metallacetylaceto-

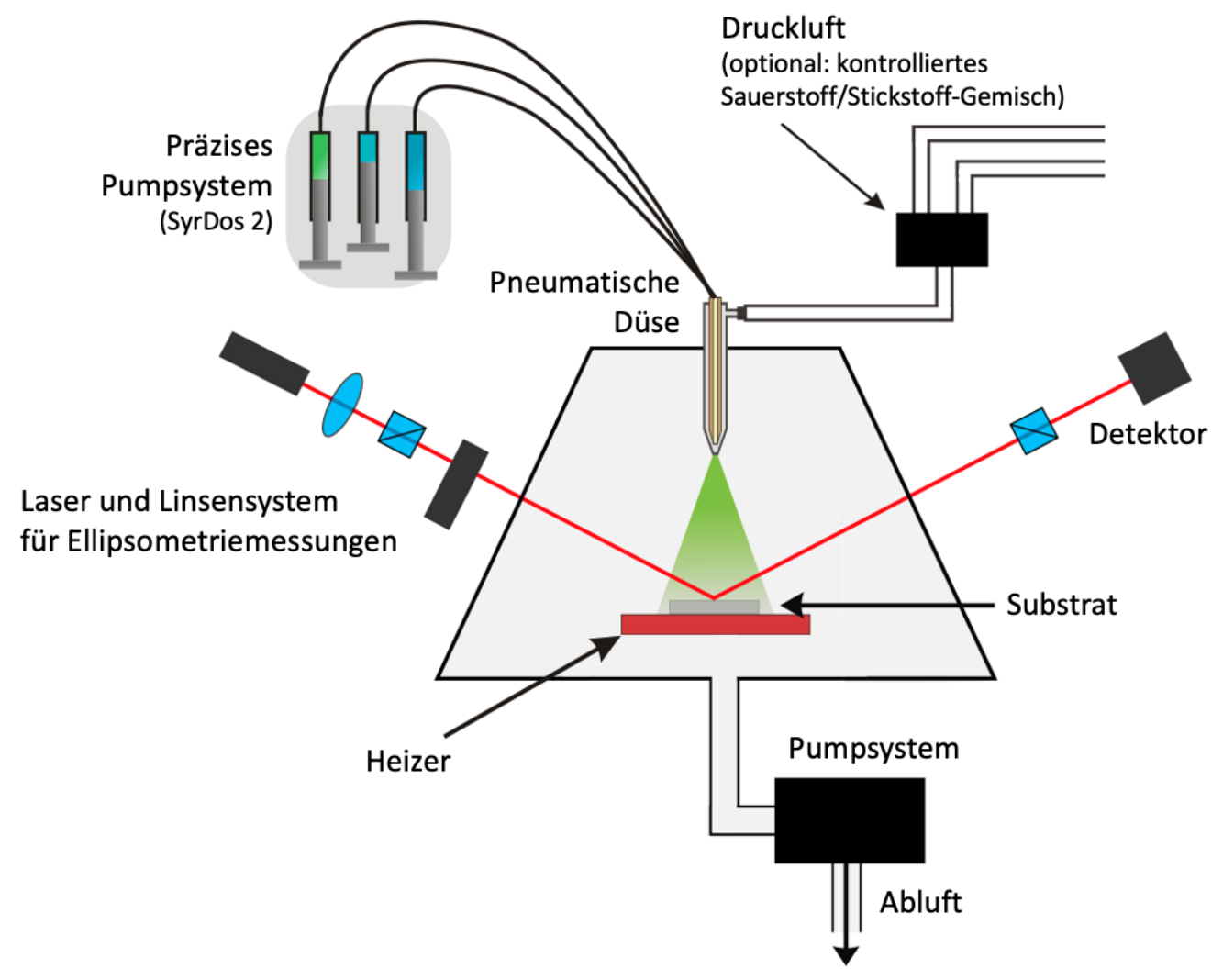

Abbildung 3.1.: Schematische Darstellung der MAD-Anlage mit in-situEllimpsometrie und kontrollierbarem Atmosphärendruck. 
nate mit dem Sauerstoff der Umgebungsatmosphäre in Verbindung treten. Dabei verbrennt der organische Bestandteil, wodurch sich das Metalloxid auf dem Substrat abscheiden kann. Am Beispiel von Nickel und Iridium sieht die Reaktion wie folgt aus:

$$
\begin{gathered}
6 \mathrm{Ni}\left(\mathrm{C}_{5} \mathrm{H}_{7} \mathrm{O}_{2}\right)_{2}+73 \mathrm{O}_{2} \rightarrow 2 \mathrm{Ni}_{3} \mathrm{O}_{4}+60 \mathrm{CO}_{2}+42 \mathrm{H}_{2} \mathrm{O} \\
4 \mathrm{Ir}\left(\mathrm{C}_{5} \mathrm{H}_{7} \mathrm{O}_{2}\right)_{3}+73 \mathrm{O}_{2} \rightarrow 4 \mathrm{IrO}_{2}+60 \mathrm{CO}_{2}+42 \mathrm{H}_{2} \mathrm{O}
\end{gathered}
$$

Trotz der ähnlichen Reaktionsgleichungen reagieren die jeweiligen Präkusoren unterschiedlich effektiv. Dies ist zum einen auf unterschieldiche kinetische und thermodynamische Prozesse während des Schichtwachstums zurückzuführen und zum anderen auf unterschiedliche Löslichkeiten in DMF und Empfindlichkeiten auf Restfeuchtigkeit bei der Lagerung. Möchte man nun ein Materialsystem aus mehreren Komponenten herstellen, weicht deshalb in der Regel das Verhältnis der jeweiligen Präkusoren vom stöchiometrichen Verhältnis in der Probe ab und muss empirisch ermittelt werden.

Die heterogene Hochtemperaturpyrolysereaktion an der Substratoberfläche in einer sauerstoffhaltigen Gasphase ist dabei das Hauptmerkmal der MAD. Ihr Vorteil gegenüber der PLD und MBE besteht darin, dass das Wachstum in der MAD stark von den Ungleichgewichtsbedingungen, die in der MBE und PLD herrschen, abweicht und sich einem Gleichgewichtszustand annähert, ähnlich dem einer Flüssigphasenepitaxie (LPE, engl.: liquid phase epitaxy) [9,63]. Der Vorteil ist eine drastische Abnahme des temperaturabhängigen Übersättigungsgrades $\alpha$ und eine damit einhergehende Zunahme der Keimbildungsclustergröße $r_{S} \approx 1 / \alpha[9,63,114,115]$. Infolgedessen ermöglichen die MAD-Wachstumsbedingungen, epitaktische Oxidfilme, Nanokomposite und Heterostrukturen von hoher Kristallqualität zu erhalten.

Je nach Anwendungsbereich kann die MAD-Technik weiter ausgebaut und durch eine Vielzahl an Komponenten erweitert werden. Einige Materialsysteme wie beispielsweise $\mathrm{VO}_{2}$ oder $\mathrm{Sr}_{2} \mathrm{FeMoO}_{6}$ benötigen zur Herstellung eine kontrollierte Einstellung des Sauerstoffpartialdruckes $p_{\mathrm{O}_{2}}$ unterhalb des atmosphärischen Partialdrucks [116-120]. Um dies zu ermöglichen wurde im Rahmen der Masterarbeit von Sven Esser [120] und der vorliegenden Arbeit 


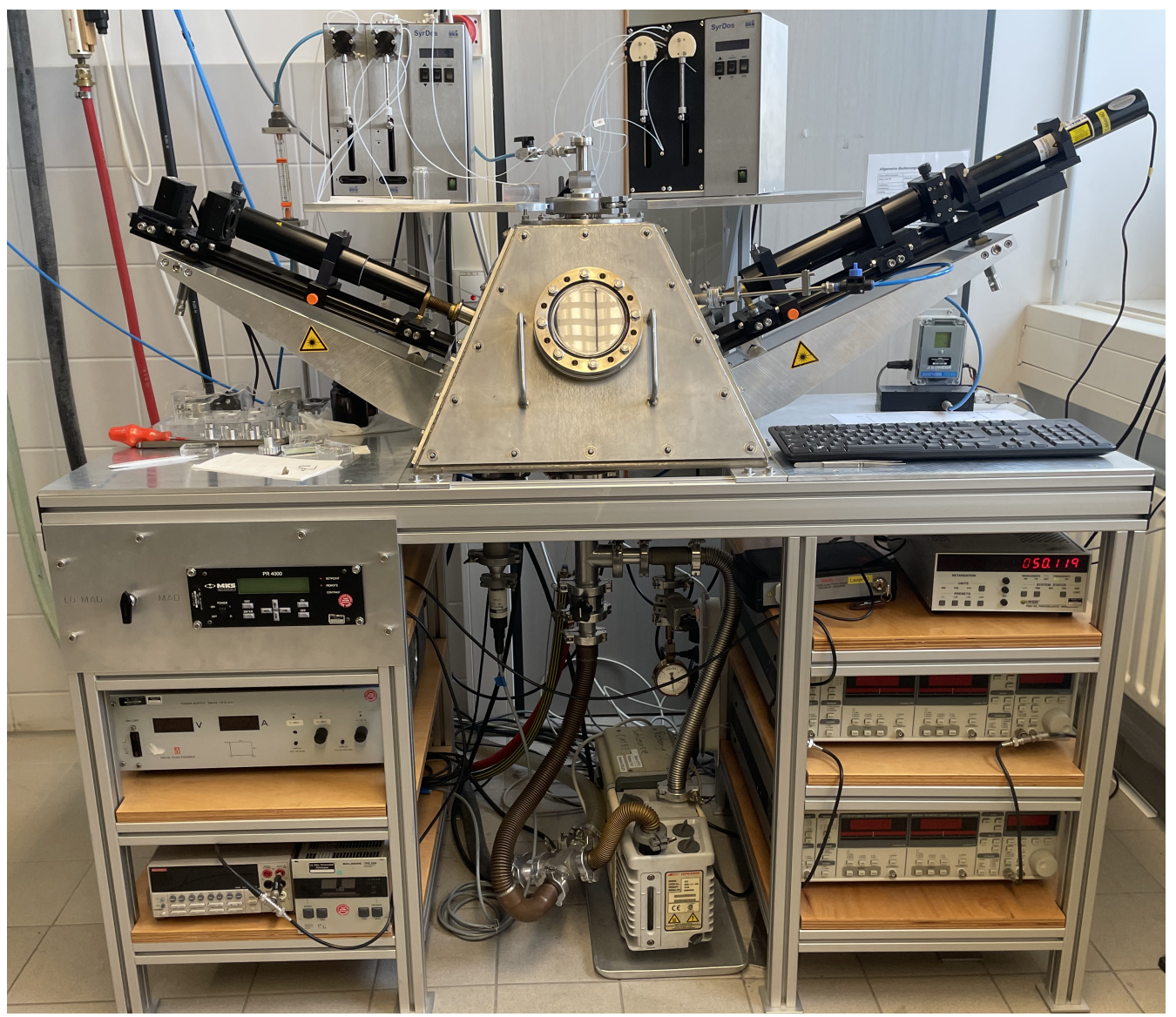

Abbildung 3.2.: Aufnahme der Pyra-MAD-Anlage, die zur Herstellung der in der Arbeit verwendeten Proben benutzt wird.

eine MAD-Anlage angefertigt, die durch ein Pumpsystem und einen Massenflusskontroller ${ }^{1}$ eine kontrollierte $\mathrm{O}_{2} / \mathrm{N}_{2}$-Atmosphäre erzeugen kann. Diese Anlage ist in Abbildung $3.2 \mathrm{zu}$ sehen, deren schematischer Aufbau bereits in Abbildung 3.1 dargestellt wird. Aufgrund ihrer Form und erweiterten Anwendungsbereiche ist sie sowohl als Pyra-MAD ${ }^{2}$ als auch als low-oxigenMAD bekannt. Um die Funktionsfähigkeit des low-oxigen-Setups zu erproben, wurden erfolgreich $\mathrm{VO}_{2}$-Proben hergestellt, die einen Metall-Isolator Übergang bei $T_{M I}=340 \mathrm{~K}$ zeigen.

Darüber hinaus lassen sich ebenfalls Depositionen unter kontrolliertem Druck

\footnotetext{
${ }^{1}$ PR4000 der Firma MKS Instruments.

${ }^{2}$ Die pyramidenartige Form ist auf den Ellipsometrie-Aufbau zurückzuführen und ermöglicht es, dass der Laserstrahl die Probe unter dem sogenannten Brewsterwinkel von $\mathrm{SrTiO}_{3}$ trifft, der im nächsten Abschnitt thematisiert wird.
} 
in einem Bereich bis zu 0,4 bar durchführen ${ }^{3}$. Versuche am Doppelperowskit $\mathrm{La}_{2} \mathrm{CoMnO}_{6}$ haben gezeigt, dass ein Differenzdruck zur Umgebung von $\Delta p=p_{\text {atm }}-p_{\text {pyra }}=0,6$ bar zu einer deutlichen Erhöhung der Wachstumsrate $v$ um $v_{\Delta p} \approx 4 \times v_{a t m}$ führt und die Schichtqualität verbessern kann. Die AFM-Aufnahme der Oberflächenmorphologie ist in Abbildung A.1 im Anhang zu finden.

Um die Herstellung feinster und auf Atomlagen genaue Übergitter zu gewährleisten, benötigt es ein sehr präzises Pumpsystem, das die Lösung in die pneumatische Düse einführt. Dafür werden an der MAD-Anlage zwei SyrDos 2 Pumpsysteme der Firma HiTec Zang verwendet, die mit einer Genauigkeit von 0,01 $\mu l$ arbeiten [121]. Die Depositionsgeschwindigkeit kann variiert werden und so die Wachstumsrate der Schicht unter normalem Atmosphärendruck von $0,03 \mathrm{~nm} / \mathrm{s}$ bis hin zu $2 \mathrm{~nm} / \mathrm{s}$ eingestellt werden ${ }^{4}$. Auf diese Weise können Heterostrukturen aus bis zu vier unterschiedlichen Materialien mit einer hohen Präzision hergestellt werden.

Neben der Deposiotionsmenge und -geschwindigkeit hat auch die Depositionstemperatur einen immensen Einfluss auf das Wachstum. Da die Oberflächentemperatur des Substrates empfindlich von der Beschaffenheit ${ }^{5}$ des SiC-Heizer abhängt, ist es notwendig, die Temperatur in regelmäßigen Abständen zu überprüfen. Dafür werden an der Pyra-MAD zwei Verfahren verwendet: zum einen wird ein Infrarot-Pyrometer der Firma Omega verwendet, das auf das Substrat gerichtet ist und zum anderen wird die Temperatur regelmäßig mit Hilfe eines doppelseitig polierten Saphirsubstrates überprüft. Dabei wird der Laserstrahl des Ellipsometrieaufbaues genutzt und dessen Intensität nach der Reflexion an der Ober- und Unterseite eines doppelseitig polierten Substrates gemessen. Durch die thermische Ausdehnung entsteht ein zuverlässig reproduzierbarer Interferenzverlauf, der direkt mit der Temperatur in Verbindung gebracht werden kann. Diese Methode ermöglicht es ebenfalls ohne viel Aufwand die Temperatureinstellungen zwischen unterschiedlichen MAD-Anlagen zu vergleichen. Abbildung A.2 im Anhang zeigt beispielhaft eine Temperaturkalibrierung der MAD-Anlage.

\footnotetext{
${ }^{3}$ Das Auswechseln der Spritzensystems hin zu einer peristaltische Pumpe würde den Anwendungsbereich auf bis unter $<0,1$ mbar erweitern.

${ }^{4}$ eine Lösungskonzentration von $0,02 \mathrm{~mol} / \mu \mathrm{l}$ vorausgesetzt.

${ }^{5}$ Widerstand und Oberflächenrauigkeit.
} 


\subsection{In-situ-Ellipsometrie}

Die in-situ-Ellipsometrie ermöglicht auf unterschiedliche Weisen, Rückschlüsse über das Wachstum und die Eigenschaften des wachsenden Materialsystems zu ziehen. Je nach Situation lassen sich der Brechungsindex einer Schicht, die Schichtdicke oder auch die Wachstumsmode bestimmen. Um diese Aussagen treffen zu können, genügt es jedoch nicht, sich ausschließlich die reinen Messdaten anzuschauen. Vielmehr müssen die Daten mit theoretischen Berechnungen verglichen werden, um zuverlässige Schlussfolgerungen aus den Messungen ziehen zu können.

Der folgende Abschnitt befasst sich aus diesem Grund damit, die theoretischen Grundlagen der Ellipsometrie zu durchleuchten, die als Ausgangspunkt für die Simulationen und das grundlegende Verständnis dienen. Anschließend wird die technische Realisierung an der MAD-Anlage vorgestellt.

\subsubsection{Theoretische Betrachtung}

Abbildung 3.3 veranschaulicht das Prinzip der Ellipsometriemessung. Ein Lichtstrahl, dessen lineare Polarisation einen Winkel von $45^{\circ}$ mit der Einfallsebene ${ }^{6}$ einschließst, wird unter dem Einfallswinkel $\Theta$ auf die Messprobe gerichtet und reflektiert. Der einfallende Strahl (i) wird dabei in zwei Komponenten zerlegt: parallel zur Einfallsebene $\left(E_{i p}\right)$ und senkrecht da$\mathrm{zu}\left(E_{i s}\right)$. Aufgrund der unterschiedlichen komplexen Reflexionskoeffizienten für den parallelen und senkrechten Anteil des Strahles, ergibt sich nach der Reflexion eine neue Polarisation des Strahls (r) mit einer gegenüber dem ursprünglichen Winkel verdrehten Hauptachse. Der Winkel zwischen der Einfallsebene und der Hauptachse des Polarisationsvektors wird als $\Psi$ und die Phasenverschiebung zwischen p- und s-Komponente als $\Delta$ bezeichnet, wie in Abbildung 3.3 dargestellt.

Die Reflexionskoeffizienten für die p- und s-polarisierte Strahlung sind über:

$$
r_{p}=\frac{E_{r p}}{E_{i p}}, r_{s}=\frac{E_{r s}}{E_{i s}}
$$

\footnotetext{
${ }^{6}$ aufgespannt durch den Normalenvektor auf die Probenoberfläche und der Propagationsrichtung des Lichtstrahls.
} 


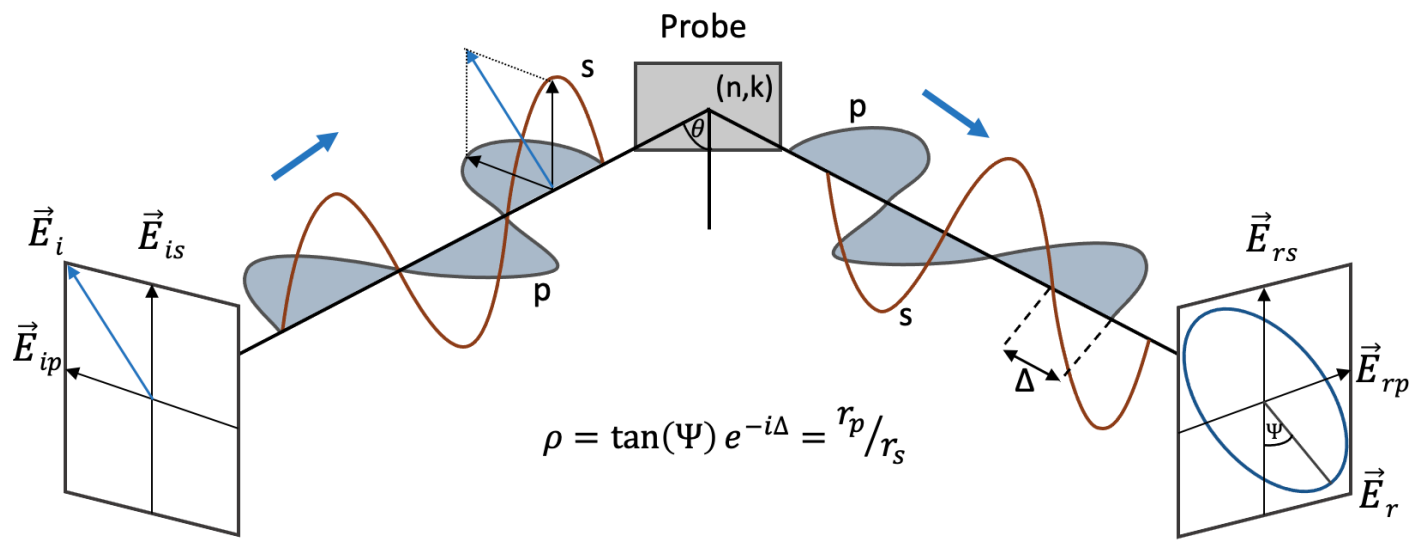

Abbildung 3.3.: Schematische Darstellung der in-situ-Ellipsometrie Messung. Ein linearpolarisierter Lichtstrahl, dessen Polarisation einen $45^{\circ}$ zur Einfallsebene der Probe hat, trifft auf die zu untersuchende Probe und wird an dieser reflektiert. Die Wechselwirkung mit der Probe bewirkt eine Phasenverschiebung $\Delta$ zwischen p- und s- Komponente und verschiebt die Polarisation des Lichtes um einen Winkel $\Psi$. Nach [27].

gegeben. Über das Verhältnis dieser Reflexionskoeffizienten $\rho$ lassen sich die Messgrößen der Ellipsometrie $(\Psi, \Delta)$ definieren [27]:

$$
\rho=\tan \Psi e^{i \Delta}=\frac{r_{p}}{r_{s}}
$$

$\Psi$ ergibt sich somit über $\Psi=\tan ^{-1}|\rho|$ und $\Delta$ stellt das Argument ${ }^{7}$ von $\rho$ dar, $\Delta=\arg (\rho)$.

Unter Anwendung der sogenannten Fresnel-Gleichungen lassen sich bei gegeben Brechungsindex der beteiligten Materialien die beiden Ellipsometrieparameter $(\Psi, \Delta)$ bestimmen und umgekehrt [27]. Für einen Lichtstrahl, der wie in Abbildung 3.4 a) aus einem Medium mit Brechungsindex $N_{i}$ in ein anderes Medium mit dem Brechungsindex $N_{t}$ übergeht, ergeben sich die Fresnel-Gleichungen für den reflektierten Strahl über:

$$
r_{p}=\frac{E_{r p}}{E_{i p}}=\frac{N_{t} \cos \Theta_{i}-N_{i} \cos \Theta_{t}}{N_{t} \cos \Theta_{i}+N_{i} \cos \Theta_{t}}, \quad r_{s}=\frac{E_{r s}}{E_{i s}}=\frac{N_{i} \cos \Theta_{i}-N_{t} \cos \Theta_{t}}{N_{i} \cos \Theta_{i}+N_{t} \cos \Theta_{t}}
$$

\footnotetext{
${ }^{7}$ bzw. den komplexen Winkel.
} 
a)

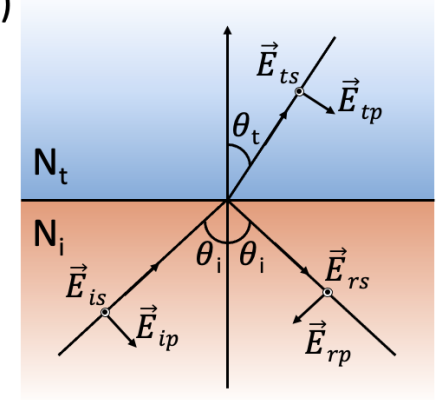

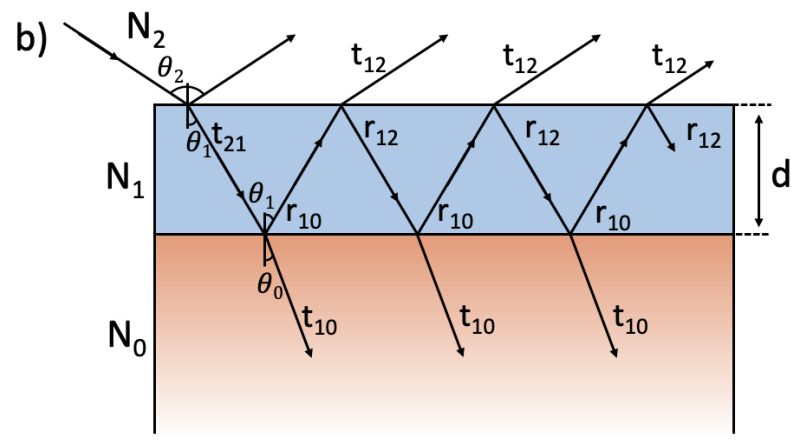

Abbildung 3.4.: a) Darstellung der relevanten Größen der elektromagnetischen Welle für die Berechnung der Fresnel-Koeffizienten. b) Illustration der Reflexions- und Transmissionskoeffizienten für eine Schicht der Dicke $d$ auf einem Substrat. Nach [27].

und für den transmitierten Strahl über:

$$
t_{p}=\frac{E_{t p}}{E_{t p}}=\frac{2 N_{i} \cos \Theta_{i}}{N_{t} \cos \Theta_{i}+N_{i} \cos \Theta_{t}}, \quad t_{s}=\frac{E_{t s}}{E_{t s}}=\frac{2 N_{i} \cos \Theta_{i}}{N_{i} \cos \Theta_{i}+N_{t} \cos \Theta_{t}} .
$$

Die genaue Herleitung der Fresnel-Gleichungen kann in [27] nachvollzogen werden. Der Winkel $\Theta_{i}$ ist über den Versuchsaufbau gegeben, während sich der Winkel $\Theta_{t}$ über das Snelliussche Brechungsgesetz bestimmen lässt:

$$
N_{t} \sin \Theta_{t}=N_{i} \sin \Theta_{i}
$$

Der Brechnungsindex $N$ eines Materials ergibt sich aus einem realen Teil $n$ und einem komplexen Teil $k, N=n-i k$. Bei einfachen Probenstrukturen wird das Amplitudenverhältnis durch $n$ beschrieben, während $k$ ein Maß für die Lichtabsorption darstellt. In Abhängigkeit des zu untersuchenden Materials, lässt sich für Medien mit verschwindendem Imaginärteil (somit vorzugsweise durchsichtige Materialien) ein Winkel finden, bei dem die Reflexion der $\mathrm{p}$-Komponente verschwindet, $r_{P}=0$. Bei diesem sogenannten Brewster-Winkel $\Theta_{B}$ kommt es beim Wachstum einer neuen Schicht auf dem Substrat zu starken Änderungen der Phase $\Delta$, womit die Sensitivität maximiert wird. Beim vorliegenden Aufbau wird der Brewster-Winkel für $\mathrm{SrTiO}_{3}$ eingestellt. Mit $N_{S T O}=2,388(\lambda=632,8 \mathrm{~nm})$ [122] und $N_{\text {Luft }}=1$ ergibt sich der Brewster-Winkel über ${ }^{8} \tan \Theta_{B}=N_{\text {STO }} / N_{\text {Luft }}$ zu $\Theta_{B} \approx 67,3^{\circ}$.

\footnotetext{
${ }^{8}$ aufgrund der nicht vorhandenen Imaginärteile für $N_{S T O}$ und $N_{L u f t}$.
} 
Abbildung 3.4 b) zeigt, dass bei der Untersuchung der Ellipsometrie an einer wachsenden Schicht mit $N_{1}$ auf einem Substrat mit $N_{2}$ die Überlagerung einer vielfachen Reflexion an den beiden vorhandenen Grenzflächen berücksichtigt werden muss. Die Ausdehnung des Substrates und der Luft mit $N_{0}$ werden als unendlich angenommen und die Schicht hat die Schichtdicke $d$. Die Reflexions- und Transmissionskoeffizienten werden dabei bei jedem Strahl weiterhin anhand der Fresnel-Gleichungen bestimmt, wobei berücksichtigt werden muss, dass während der Propagation durch die Schicht die elektromagnetische Welle eine Phasenänderung um:

$$
\beta=\frac{2 \pi d}{\lambda} N_{1} \cos \Theta_{1}
$$

erfährt [27]. Der endgültige Reflexionskoeffizient $r_{210}$ ergibt sich somit über die Beiträge aller mehrfach reflektierten Strahlen, die phasenrichtig aufaddiert werden müssen. Dabei entsteht eine geometrische Reihe, die sich zu:

$$
r_{210}=\frac{r_{21, p / s}+r_{10, p / s} e^{-i 2 \beta}}{1+r_{21, p / s} r_{10, p / s} e^{-i 2 \beta}}
$$

zusammenfassen lässt ${ }^{9}$.

Möchte man Heterostrukturen aus mehreren Schichten untersuchen, so kann man das vorgestellte Prinzip verwenden und iterativ vorgehen. Dabei bestimmt man die Koeffizienten $r_{210}$ und $t_{210}$ zunächst für die unterste Lage und fasst diese dann mit dem Substrat zu einem neuen virtuellen Substrat zusammen. Nun können die Koeffizienten der darüber liegenden Schicht bestimmt werden und anschließend ebenfalls zusammengefasst werden. Dieses Verfahren wiederholt man, bis man bei der obersten Schicht angelangt ist.

\footnotetext{
${ }^{9}$ Analog erhält man für den Transmissionskoeffizienten $t_{210}=\frac{t_{21, p / s} t_{10, p / s} e^{-i \beta}}{1+r_{21, p / s} r_{10, p / s} e^{-i 2 \beta}}$.
} 


\subsubsection{Technische Realisierung}

Die Ellipsometrie-Messung wird an der MAD nach dem Prinzip der Phasenmodulation durch einen photoelastischen Modulator konzipiert und ist schematisch in Abbildung 3.5 dargestellt [27,123]. Dieser Aufbau wurde mit dem Konzept der MAD auf ähnliche Weise erstmals im Rahmen der Doktorarbeit von Markus Jungbauer an einer offenen MAD-Anlage (MAD 3) realisiert [124]. Als Lichtquelle wird ein linear polarisierter Helium-Neon-Laser mit der Wellenlänge $\lambda=632,8 \mathrm{~nm}$ verwendet. Um die lineare Polarisation des Lichtes zusätzlich zu verstärken durchläuft das Licht einen Polarisator, dessen Durchlassrichtung um $45^{\circ}$ gegen die Einfallsebene verkippt ist.

Der phasenmodulierte Ellipsometrieaufbau basiert darauf, dass zwischen der p- und s-Komponente des Polarisationsvektors des Lichtes eine oszillierende Phasenverschiebung, $\delta(t)=\delta_{0} \sin (\omega t)$ existiert. Um diese Phasenverschiebung zu erzeugen, wird ein sogenannter photoelastischen Modulator (PEM) verwendet. Das PEM besteht aus einem Quarzglas-Quader, der auf einem piezoelektrischen Quarzkristall aufgeklebt ist. Durch Anlegen einer Wechselspannung von $50 \mathrm{kHz}$ lässt sich im Kristall eine elastische Schwingung erzeugen, die zu einer Variation der lokalen Dichte und zu einer Änderung des Brechungsindex führt. Durch die Querkontraktion ist die Dichteänderung

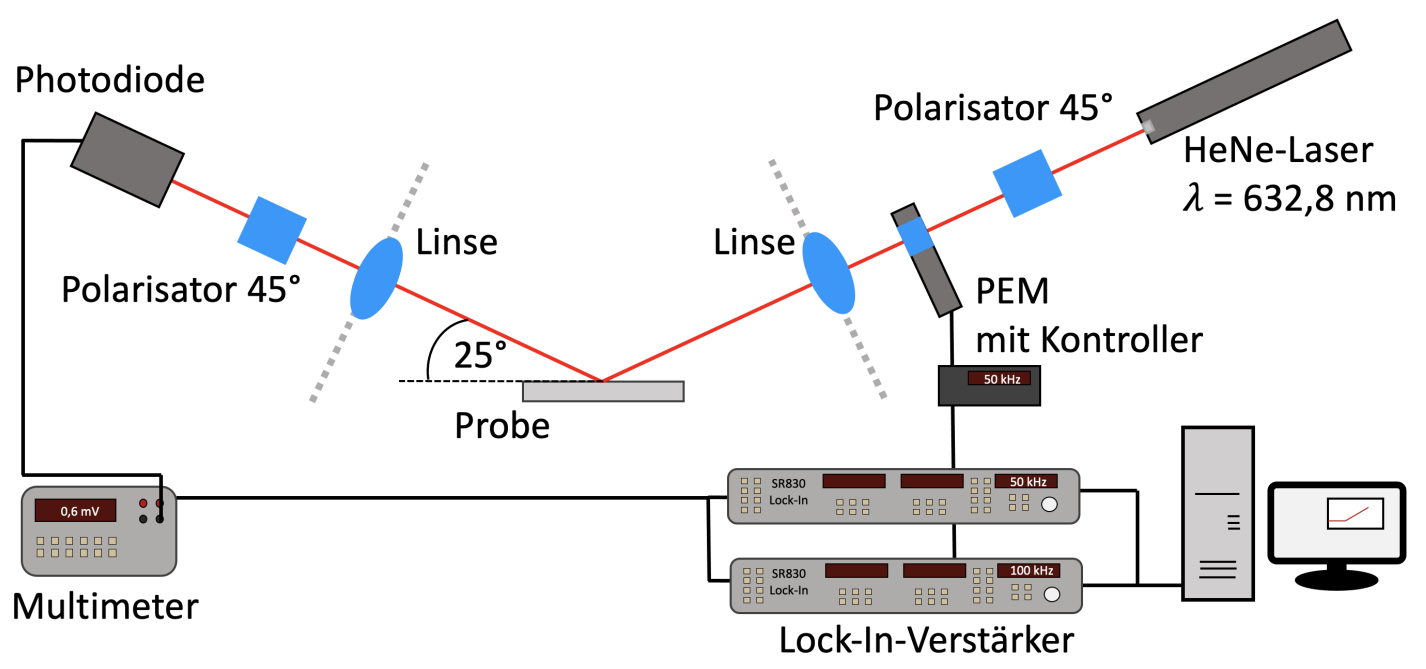

Abbildung 3.5.: Schematische Darstellung des Aufbaues zur phasenmodulierten Ellipsometrie-Messung an der Pyra-MAD. 
senkrecht zur angelegten Schwingebene anders als parallel dazu, wodurch zwischen den Komponenten der elektromagnetischen Welle mit Polarisation parallel und senkrecht zur angeregten Schwingungsebene die gewünschte Phasenverschiebung induziert wird. Die Amplitude $\delta_{0}$ lässt sich über die angelegte Spannung an dem piezoelektrischen Kristall einstellen [125].

Das so modulierte Licht wird nun mittels einer Linse auf die Probe fokussiert und tritt so in die MAD-Kammer ein. Dieser Aufbau ermöglicht es, dass die Pyra-MAD auch unter Vakuumbedingungen benutzt werden kann. Unter dem Winkel von 65, der nahe dem Brester Winkel von $\mathrm{SrTiO}_{3}$ liegt, tritt das Licht nun auf die Probe und verlässt auf gleiche Weise über eine Linse die MAD-Kammer. Ein letztes Mal durchläuft das Licht einen Polarisator, der auch hier einen $45^{\circ}$-Winkel mit der Einfallsebene einschließt, und wird schließlich von einer Si-Photodiode erfasst.

Zur mathematischen Beschreibung der Veränderung des Polarisationszustandes und der daraus resultierenden Intensität lässt sich der Jones-Formalismus verwenden. Bei der verwendeten Konfiguration ergibt sich so folgende Intensität:

$$
\begin{aligned}
I(t)=\left|\vec{E}_{t o t}\right|^{2}=I_{0}( & +J_{0}\left(\delta_{0}\right) I_{0} \sin (2 \Psi) \cos (\Delta) \\
& +2 J_{1}\left(\delta_{0}\right) I_{0} \sin (2 \Psi) \sin (\Delta) \sin (\omega t) \\
& \left.+2 J_{2}\left(\delta_{0}\right) I_{0} \sin (2 \Psi) \cos (\Delta) \cos (2 \omega t)\right) .
\end{aligned}
$$

Dies kann in [27] genauer nachvollzogen werden. $J_{n}$ sind dabei die Besselfunktionen. Die Amplitudenänderung des Modulators ist im gegebenen Aufbau auf $\delta_{0}=139^{\circ}$ eingestellt. Numerisch ergibt sich dann für die Besselfunktionen $J_{0}\left(\delta_{0}\right)=0,011 \approx 0, J_{1}\left(\delta_{0}\right)=0,515$ und $J\left(\delta_{0}\right)_{2}=0,435$. Dadurch vereinfacht sich die Formel für die Intensität zu:

$$
I(t)=\left|\vec{E}_{t o t}\right|^{2}=I_{0}+I_{\omega}(t) \sin (\omega t)+I_{2 \omega}(t) \cos (2 \omega t)
$$

mit:

$$
\begin{gathered}
I_{\omega}=2 J_{1}\left(\delta_{0}\right) I_{0} \sin (2 \Psi) \sin (\Delta) \\
I_{2 \omega}=2 J_{2}\left(\delta_{0}\right) I_{0} \sin (2 \Psi) \cos (\Delta) .
\end{gathered}
$$


Aus den Messgrößen $I_{0}, I_{\omega}$ und $I_{2 \omega}$ lassen sich dann die Messgrößen der Ellipsometrie $\Delta$ und $\Psi$ berechnen über:

$$
\Delta=\tan ^{-1}\left(\frac{I_{\omega} J_{2}\left(\delta_{0}\right)}{I_{2 \omega} J_{1}\left(\delta_{0}\right)}\right) \quad \text { und } \quad \Psi=\frac{1}{2} \sin ^{-1}\left(\frac{I_{\omega}}{2 J_{1}\left(\delta_{0}\right) I_{0} \sin (\Delta)}\right)
$$

Im Aufbau der vorliegenden Arbeit wird das Spannungssignal der Photodiode, das proportional zur Intensität $I_{0}$ ist, mit einem Voltmeter (Keithley 2000 [126]) gemessen und die zwei Harmonischen $I_{\omega}$ und $I_{2 \omega}$ werden unter Benutzung des Referenzsignales vom Kontroller des PEM von zwei LockIn-Verstärkern (Model SR830 der Firma Standford Research Systems [127]) aufgezeichnet. Schließlich erfolgt die Messdatenaufnahme mit demselben Computerprogramm, das auch die Dosierung der Präkursorlösungen mit den Spritzendosierern steuert. Der Aufbau der Ellipsometrie, die Programmierung der Ansteuerungen der einzelnen Komponenten und die Implementierung der Signalverarbeitung in das MAD-Programm wurden im Rahmen der vorliegenden Arbeit durchgeführt. 
$\overline{\text { Kapitel }}$

\section{Charakterisierung}

Nachdem nun ausführlich auf die Herstellung der Probensysteme eingegangen wurde, soll sich das folgende Kapitel den Charakterisierungsmethoden widmen. Die Besonderheit der Übergangsmetalloxide liegt in ihren vielseitigen Eigenschaften, die es zu untersuchen gilt. Dafür benötigt es entsprechend auch eine Vielzahl verschiedener Methoden, die Einblick in die unterschiedlichen Materialeigenschaften liefern. Grundlage für das Verständnis der physikalischen Phänomene in einem Material bilden die strukturellen Eigenschaften, die mittels Rasterkraftmikroskopie, Transmissionselektronenmikroskopie, Röntgenbeugung und -reflektrometrie untersucht werden. Zusätzlich erlaubt die Raman-Spektroskopie mit Hilfe von spektraler Untersuchung inelastischer Streuung von Licht an Festkörpern weitere Rückschlüsse auf die Struktur des Materials. Darauf aufbauend können anschließend temperatur- und feldabhängige Messungen der magnetischen und elektrischen Eigenschaften mittels MPMS (engl.: magnetic property measurement system) und eines PPMS-Gerätes (engl.: physical property measurement system) durchgeführt werden. Weiterführend werden in Zusammenarbeit mit der Gruppe um Philipp Gegenwart an der Universität Augsburg Hallmessungen zur Untersuchung des anomalen und topologischen Halleffekts durchgeführt. 


\subsection{Rasterkraftmikroskopie}

Die Oberflächenmorphologie von Dünnfilmen lässt wichtige Rückschlüsse über das Wachstum der Schichten zu. Aus diesem Grund werden die Oberflächenmorphologie und die Rauigkeit der hergestellten Schichten mithilfe eines Rasterkraftmikroskops (AFM, engl.: atomic force microscope) der Firma Burker AXS untersucht [128].

Das grundlegende Messprinzip ist in Abbildung 4.1 dargestellt. Für die Messung wird ein Cantilever (ein Biegebalken, wie in Abbildung $4.1 \mathrm{zu}$ sehen) in die Nähe der Probenoberfläche gebracht, an dessen Unterseite sich eine scharfe Spitze befindet, die im Idealfall an ihrem Ende aus nur einem Frontatom besteht. Über die Wechselwirkung des Frontatomes der Spitze mit den Atomen der Probenoberfläche verbiegt sich der Cantilever. In dem ein Laserstrahl auf die Rückseite des Cantilevers gestrahlt wird und die Reflektion von einer positionssensitiven Photodiode detektiert wird, lässt sich die Auslenkung aus der Nulllage sowohl in vertikaler als auch in horizontaler Richtung messen [129]. Über einen Controller wird das detektierte Signal der Photodiode dem Regelkreis als Rückkopplung zurückgeführt und mit Hilfe von Piezoelementen das Abrastern des Cantilevers in x-, y- und zRichtung gesteuert [128]. Geeignete Auswertungsprogramme (in diesem Fall Gwyddion) verarbeiten das detektierte Signal und konstruieren die Oberflä-

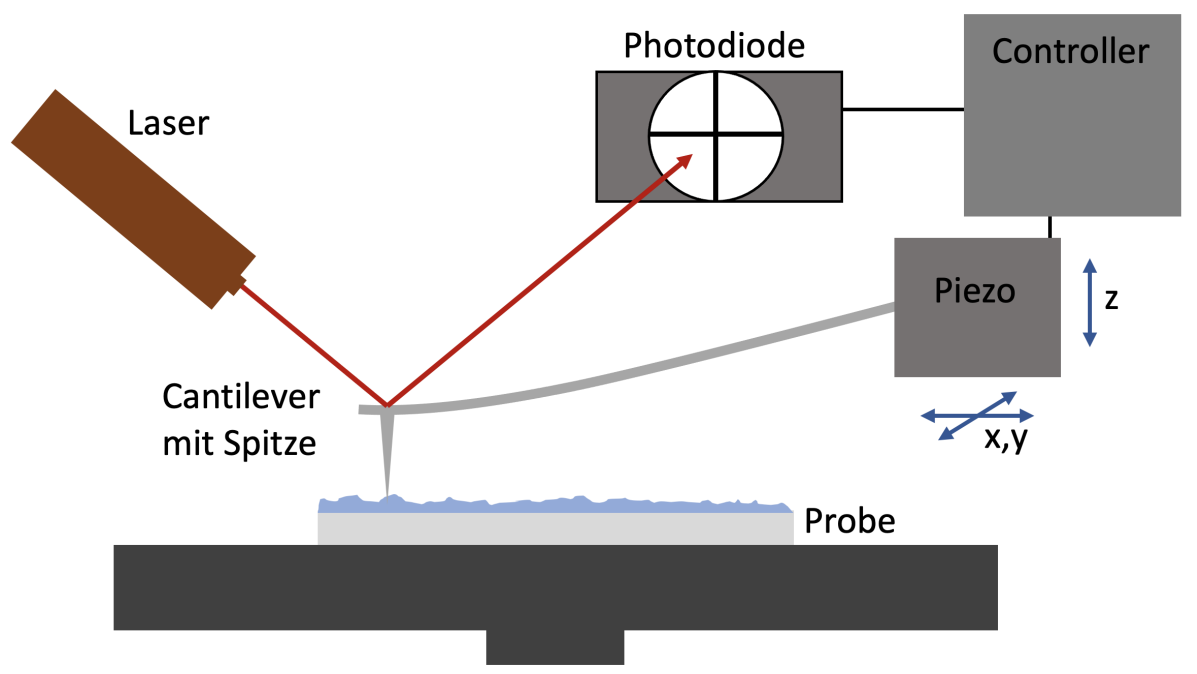

Abbildung 4.1.: Schematische Darstellung der Funktionsprinzipes des AFM. 
chengeometrie [130]. Die Morphologie kann mit dem AFM in verschiedenen Betriebsarten aufgenommen werden und wird in der vorliegenden Arbeit im Kontakt-Modus (engl.: contact mode) benutzt. Dabei befindet sich die Spitze dauerhaft im Kontakt mit der Probenoberfläche und rastert diese systematisch ab. Die quadratische Rauigkeit $R_{q}$, (bzw. RMS, engl.: root mean squared roughness) wird in der Arbeit als Maß für die Oberflächenqualität verwendet. Diese Größe errechnet sich aus [131]:

$$
R_{q}=\sqrt{\frac{\sum_{i=1}^{N}\left(Z_{i}-Z\right)^{2}}{N}} .
$$

Hierbei ist $\mathrm{Z}$ der durchschnittliche relative Höhenwert, $Z_{i}$ die einzelnen Höhenwerte an den jeweiligen Rasterpunkten und N die Gesamtanzahl an Rasterpunkten auf dem betrachteten Ausschnitt der Probenoberfläche.

\subsection{Transmissionselektronenmikroskopie}

Um die Struktureigenschaften wie der B-Platzordnung in Doppelperowskiten zu erhalten, sind Untersuchungen mit dem Transmissionselektronenmikroskop (TEM, engl.: transmission electron microscope) in Verbindung mit Elektronenenergieverlustspektroskopie (EELS, engl.: electron energy loss spectroscopy) oft notwendig. Allerdings handelt es sich dabei um eine Untersuchungsmethode, die mit einem vergleichbar hohem Arbeitsaufwand verbunden ist, weshalb nur wenige ausgewählte Proben auf diese Weise untersucht werden.

Die vorgestellten Aufnahmen stammen von Geräten des Types FEI Titan 80-300 G2 mit einer Beschleunigungsspannung von bis zu $U=300 \mathrm{kV}$ und wurden von 1. Vladimir Roddatis sowohl im Institut für Materialphysik der Georg-August-Universität Göttingen als auch im Helmholtz-Zentrum Potsdam und 2. von Robert Gruhl aus dem Institut für Experimentalphysik VI der Universität Augsburg erstellt.

Mit dem TEM ist es möglich, Bildaufnahmen der atomaren Struktur mit einer Auflösung von < 0,2 nm zu erreichen [132-134]. Dafür wird ein Elektronenstrahl unter Verwendung einer elektromagnetischen Optik auf das 
Material fokussiert, welcher dann die Probe durchdringt. Die Elektronen des Elektronenstrahls wechselwirken über elastische Streuung (RutherfordStreuung) an den Atomkernen und durch inelastische Streuprozesse an den Elektronen der Atome und ermöglichen so die Bildgebung der atomaren Struktur [133]. Damit die Elektronen die Probe komplett durchdringen können, muss eine dünne Lamelle in Querschnittsrichtung aus einer Probe mit einer Dicke von etwa 10 bis $100 \mathrm{~nm}$ ausgeschnitten werden und die Beschleunigungsspannung der Elektronen zwischen 100 und $500 \mathrm{kV}$ liegen. Die dünnen Lamellen schneidet man mit Hilfe eines fokussierten Ionenstrahls (FIB, engl.: focused ion beam) aus der Probe aus [132-134]. Die Bildaufnahme erfolgt schließlich über einen Detektor.

In Abhängigkeit des Ablenkungswinkels unterscheidet man die detektierten Aufnahmen. Unter dem Begriff Dunkelfeld (HAADF, engl.: high-angle annular dark field) werden Aufnahmen besonders stark abgelenkter Elektronen zusammengefasst. Dieser Modus ermöglicht eine Differenzierung der chemischen Elemente in der atomaren Probenstruktur, da die Signalintensität ungefähr proportional zum Quadrat der Ordnungszahl $Z$ ist $[132,133]$. Aufnahmen der abgelenkten Elektronen, die nur in einem kleinen Winkel abgelenkt werden, werden unter dem Begriff Hellfeld (BF, engl.: bright-field) zusammengefasst. Im Hellfeld erscheinen Bereiche mit hoher Dichte sehr dunkel, während sie im Dunkelfeld eine hohe Helligkeit aufweisen.

Die verwendeten TEM-Anlagen verfügen über die Fähigkeit aus der elektronischen Anregung Elementdetektion durchzuführen (EELS-Messungen) . Dabei wird die Energieverteilung der inelastisch gestreuten Elektronen analysiert, woraus man aus der Identifikation der auftretenden Linien auf die lokale chemische Zusammensetzung der Probe schließen kann. Diese Methode ist insbesondere bei der Feststellung der B-Platzordnung in Doppelperowskiten maßgeblich $[132,134]$. 


\subsection{Röntgendiffraktometrie}

Röntgendiffraktometrie lautet im Englischen $X$-ray diffraction und wird somit für üblich mit XRD abgekürzt. XRD-Messungen dienen der strukturellen Bestimmung der hergestellten Schichten über die Beugung von Röntgenstrahlung an einem dreidimensionalen Kristallgitter und werden im Rahmen der vorliegenden Arbeit mit einem Röntgendiffraktometer D8 Advance von Bruker AXS durchgeführt.

Als Röntgenquelle ist eine Kupferanode eingebaut, die mit optischen Elementen ausgestattet ist, um eine monochromatische $\mathrm{Cu} K_{\alpha}$-Linie zu erzeugen. Allerdings spaltet sich die $\mathrm{Cu} K_{\alpha}$-Linie aufgrund der Spin-BahnKopplung in zwei separate $\mathrm{Cu} K_{\alpha, i}$-Linien auf mit den Wellenlängen $\lambda_{K_{\alpha, 1}}=$ $1,54056 \AA$ und $\lambda_{K_{\alpha, 2}}=1,54439 \AA$, die gemeinsam eine mittlere Wellenlänge von $\bar{\lambda}_{K_{\alpha}}=1,54184 \AA$ ergeben. Die Wellenlänge befindet sich somit in der Größenordnung der Atomabstände im Kristallgitter, was für die Beugung am Kristall vorausgesetzt wird.

Die Messungen werden in der Bragg-Brentano-Geometrie durchgeführt, in der der Röntgenstrahl unter einem Winkel $\theta$ auf die Probe auftrifft und der Detektor unter dem doppelten Einfallswinkel $2 \theta$ das Signal aufzeichnet. Wie in Abbildung 4.2 a) zu sehen ist, ist im Zentrum des Führungskreises von Röntgenröhre und Detektor die Probe platziert und wird mit der halben Winkelgeschwindigkeit des Detektors gedreht. Der Versuchsaufbau erlaubt zusätzlich unerwünschte Abweichungen $\Delta$ zwischen $\theta$ und $2 \theta$ mit zu berücksichtigen $^{1}$. In diesem gekoppelten Modus wird nun die gemessene Intensität $I$ der Strahlung am Detektor als Funktion des Winkels $2 \theta$ zur Röntgenröhre gemessen und ergibt so das Beugungsbild.

Um das Beugungsbild zu erklären, betrachtet man das Kristallgitter zunächst als eine starre Anordnung von Gitteratomen in Form von punktförmigen Streuzentren mit periodischen Abständen in einem dreidimensionalen Gitter. Trifft die Röntgenstrahlung auf die Gitteratome, werden diese an deren Elektronenhülle gestreut, wie in Abbildung 4.2 b) dargestellt. Die ge-

\footnotetext{
${ }^{1}$ Durch Substratfehlschnitte oder kleine Partikel unter der Probe kann die Probenoberfläche leicht verkippt sein.
} 
a)

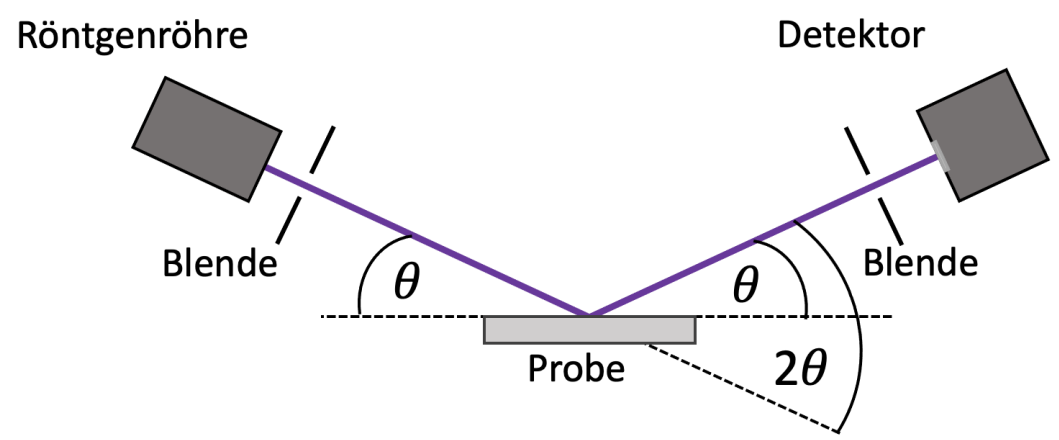

b)

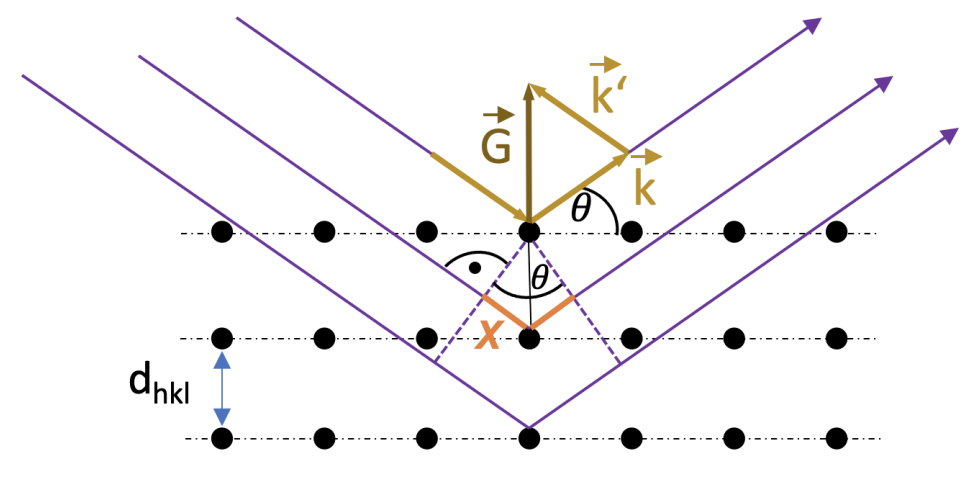

Abbildung 4.2.: Darstellung a) der XRD-Messanordnung in der BraggBrentano-Geometrie und b) der Beugungsgeometrie zur Herleitung der Bragg-Gleichung. Angelehnt an [135].

streute Strahlung der Gitteratome der unterschiedlichen Netzebenen interferiert miteinander und liefert in Abhängigkeit des Atomabstandes und des Einfallswinkels der Strahlung ein Beugungsbild aus konstruktiver und destruktiver Überlagerung. Konstruktive Interferenz tritt auf, wenn der Gangunterschied $x$ zwischen den Strahlen der unterschiedlichen Netzebenen ein ganzzahliges Vielfaches der Wellenlänge der Röntgenstrahlung ist, woraus die Bragg-Bedingung für konstruktive Interferenz folgt [107, 135-137]:

$$
2 d_{h k l} \sin (\theta)=n \lambda,
$$

Der Abstand der Ebenen zueinander wird über $d_{h k l}$ beschrieben(siehe Abbildung 4.2 b) ) und die ganze Zahl $n$ gibt die Ordnung der Reflexion an. $(h, k, l)$ stellen die Millerschen Indizes dar. Daraus lässt sich für ein kubisches 
Kristallsystem aus dem Reflex $(h k l)$, dem Einfallswinkel $\theta$ und der Wellenlänge der Strahlung $\lambda$ für $n=1$ die kubische Gitterkonstante $a$ errechnen über $[136,138]$ :

$$
a=\frac{\lambda}{2 \sin (\theta)} \sqrt{h^{2}+k^{2}+l^{2}}
$$

Will man die relative Intensität der Reflexe bestimmen, muss zusätzlich die Verteilung der Atome anhand der Basis und dem Streuvermögen der Basisatome mit berücksichtigt werden $[137,138]$. Die Intensität verhält sich proportional zu dem Betragsquadrat des Strukturfaktors $F_{h k l}$, welcher eine Fouriertransformation der Elektronendichte in der Elementarzelle der Kristallstruktur darstellt. Dieser muss über sämtliche atomare Streufaktoren ${ }^{2}$ $f_{i}(\vec{K})$ der Basisatome i bezüglich ihrer Verteilung in der Elementarzelle der Kristallstruktur aufsummiert werden [137]:

$$
F_{h k l}=\sum_{i=1}^{n} f_{i}(\vec{K}) e^{i \vec{K} \cdot \overrightarrow{r_{i}}}=\sum_{i=1}^{n} f_{i}(\vec{K}) e^{i \vec{G} \cdot \overrightarrow{r_{i}}}
$$

$\vec{G}$ stellt dabei den reziproken Gittervektor dar. Die Gleichung zeigt, dass gemäß der Laue-Bedingung der Streuvektor $\vec{K}$ für die Beobachtung konstruktiver Interferenz ein Vektor des reziproken Gitters sein muss, $\vec{K}=\overrightarrow{k^{\prime}}-\vec{k}=\vec{G}$ $[107,135,136]$.

Die eigentliche Intensität der einzelnen Reflexe wird darüber hinaus durch weitere Faktoren beeinflusst, weshalb zusätzlich ein Skalierungsfaktor $K$ und der Lorenz-Polarisierungsfaktor LP mit in die Berechnung der relativen Intensität $I_{\text {rel. }}$ einfließen [137]:

$$
I_{\text {rel. }}(h k l)=K \cdot\left|F_{h k l}\right|^{2} \cdot L P=K \cdot\left|F_{h k l}\right|^{2} \cdot\left(\frac{1+\cos ^{2}(2 \theta)}{\sin ^{2}(\theta) \cos (\theta)}\right) .
$$

Der Skalierungsfaktor $K$ bezieht dabei Probeneigenschaften wie Volumen und Absorptionsfaktor mit ein, während der Lorentz-Polarisationsfaktor LP die Winkel- und Polarisationsabhängigkeit der gestreuten Röntgenstrahlung berücksichtigt (Thomson-Streuung) [137,139]. Zuletzt muss die Temperatu-

\footnotetext{
${ }^{2}$ Diese hängen von der inneren Struktur und Elektronenzahl des jeweiligen Atomrumpfes ab. Sie sind ein Maß für das Streuvermögen der Atome in der Einheitszelle [136].
} 
rabhängigkeit der Streuung am Gitter mit betrachtet werden, die über den Debye-Waller-Faktor $D_{h k l}$ beschrieben wird [136,140,141]:

$$
I(h k l)=I_{0} \cdot D_{h k l}=I_{0} \cdot \exp \left(-\frac{k_{B} T}{M \omega^{2}} G^{2}\right)
$$

$I_{0}$ bezeichnet die Reflexintensität für ein starres Gitter, $M$ ist die Atommasse, $\vec{G}$ ist der reziproke Gittervektor des Reflexes $(h k l)$ und $\omega$ die Kreisfrequenz der harmonisch genäherten Gitterschwingung.

\subsection{Röntgenreflektrometrie}

Während die Röntgendiffraktometrie die Streuung an den einzelnen Gitterebenen betrachtet, wird in der Röntgenreflektrometrie (XRR, engl:: $X$ ray reflection) die Reflexion von Röntgenstrahlung an den Grenzflächen von Medien mit unterschiedlichen Brechungsindizes $n_{i}$ betrachtet. Aus den Messungen lassen sich die Gesamtschichtdicke $d$ eines Dünnfilmes bis hin zu individuellen Schichtdicken und deren Grenzflächenrauigkeiten in Übergittern bestimmen.

Die Messungen werden ebenfalls in der Bragg-Brentano-Geometrie wie die XRD-Messungen mit dem selben Röntgendiffraktometer durchgeführt. Jedoch werden hier die Messungen bei deutlich kleineren Winkeln $\theta$ durchgeführt, $\approx 0,4^{\circ}<\theta<10^{\circ}$. Unterhalb eines kritischen Winkels $\theta_{C} \approx 0,4^{\circ}$ kommt es zu einer Totalreflexion an der Schichtoberfläche. Für größere Winkel wird ein Teil der Röntgenstrahlung gemäß des Snelliusschen Brechungsgesetzes und der Fresnelschen Gleichungen in die Schicht hinein gebrochen und an den Grenzflächen der einzelnen Schichten und dem Substrat reflektiert, sodass es nach der Bragg-Bedingung zu einer konstruktiven oder destruktiven Überlagerung kommt.

Unter Verwendung der Parratt-Formalismus lassen sich neben der Schichtdicke auch die Rauigkeit zwischen Schicht und Substrat aber auch zwischen den einzelnen Schichten in einem Übergitter bestimmen [142]. Die Auswertung der XRR-Messungen wird mit dem Programm GenX [143] durchgeführt, indem die Messungen für das Schichtsystem simuliert und anschließend mit der Messungen verglichen und angepasst werden. 


\subsection{Raman-Spektroskopie}

Neben den vorgestellten Methoden lassen sich auch über das Phononenspektrum des Materialsystemes Rückschlüsse auf die strukturellen Eigenschaften ableiten. Zugang zu diesen Informationen bietet dabei die sogenannte Raman-Spektroskopie, die auf inelastischer Streuung von monochromatischem Licht an einem Streumedium basiert [144-146]. Dabei wird im Raman-Spektrometer Laserlicht auf eine Probe fokussiert und das rückgestreute Licht analysiert. Währenddessen kommt es hauptsächlich zu elastischer Streuung des Lichtes, der sogenannten Rayleight-Streuung, die die gleiche Anregerfrequenz wie der Laser besitzt. Ein Bruchteil der Photonen wechselwirkt jedoch inelastisch mit der Probe, sodass es zu einem Impulsübertrag zwischen den Photonen und (im Fall von Festkörpern) den Phononen kommt, der auf zwei Weisen realisiert werden kann [147,148]:

\section{Stokes-Streuung}

Durch das Photon kommt es zur Anregung der Gitterschwingung, die sich hinterher in einem höherem Energiezustand befindet. Für das gestreute Licht kommt es somit zu einer Verschiebung zu kleineren Frequenzen.

\section{Anti-Stokes-Streuung}

Hierbei kommt es zu einem Energieübertrag von den Gitterschwingungen auf das gestreute Licht und somit zu einer Verschiebung zu höheren Frequenzen.

Die Vermittlung der Streuprozesse erfolgt dabei über virtuelle Zustände. Dabei werden die Schwingungszustände der Probe nach Wechselwirkung mit dem Photon in einen instabilen Zustand angehoben, bevor sie wieder in die unteren Zustände fallen und so ein weiteres Photon emittieren. Dieser Prozess ist im sogenannten Jablonski-Diagramm in Abbildung 4.3 a) und das dazugehörige Spektrum in b) dargestellt [147, 148].

Um den Raman-Effekt der inelastischen Streuung von Licht an Materie zu beschreiben, kann man zunächst klassisch die Wechselwirkung zwischen dem 


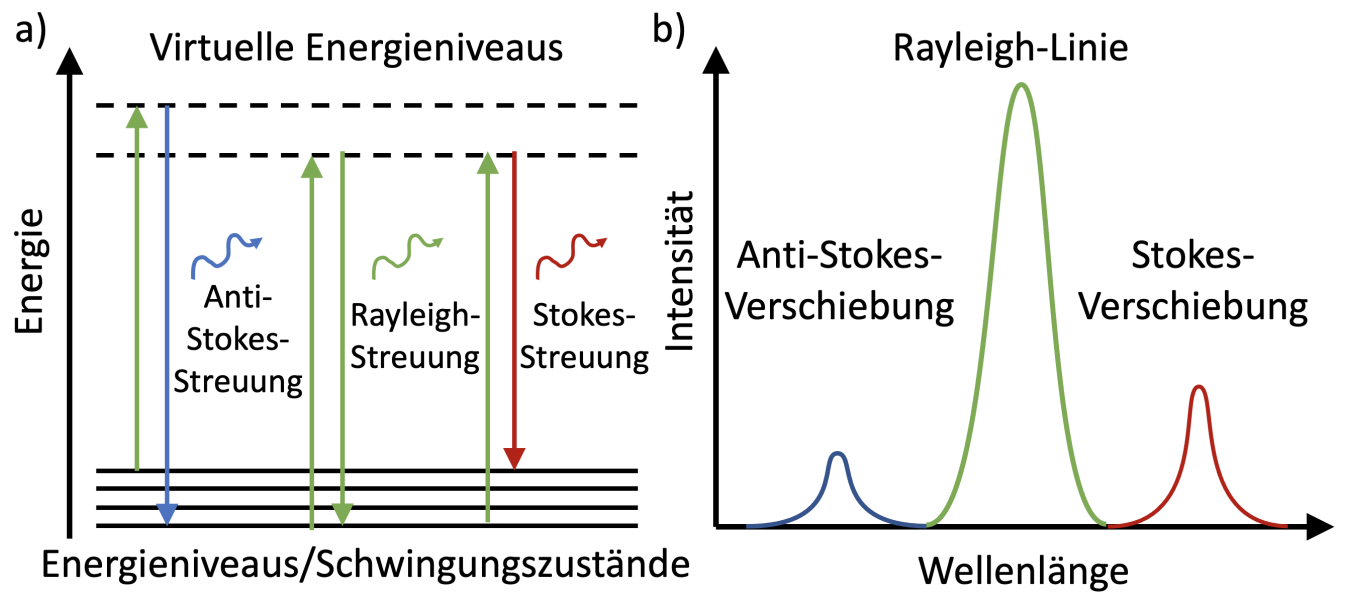

Abbildung 4.3.: a) Schematische Darstellung der Rayleigh und der RamanStreuprozesse und b) die schematische Darstellung des dazugehörigen Spektrums. Nach dem Beispiel aus [148].

oszillierenden elektromagnetischen Feld des einfallenden Lichts und des Gitters betrachten. Die theoretische Herleitung kann in $[147,148]$ nachgelesen werden und ermöglicht es, Vorhersagen über die Aktivität von Ramenmoden und dessen Intensität zu treffen.

Aus dieser Betrachtung ergibt sich zusätzlich, dass mit Hilfe von polarisationsabhängiger Raman-Spektroskopie die Intensität bestimmter Ramanmoden verändert werden kann. Dabei wird die lineare Polarisation des Laserlichts systematisch durch einen Polarisator vorgegeben und die Polarisationskomponenten des gestreuten Lichts mithilfe eines Analysators betrachtet. Je nach Konfiguration des Polarisators und Analysators ergeben sich unterschiedliche Intensitäten für materialspezifische Ramanmoden, die von der Kristallstruktur, der Raumgruppe und der Symmetrie der Moden abhängen, wie bereits an den $\mathrm{DP} \mathrm{La}_{2}(\mathrm{Ni}, \mathrm{Co}) \mathrm{MnO}_{6}$ gezeigt wurde $[56,59,62]$. Somit können über die polarisationsabhängige Raman-Spektroskopie weitere Informationen über die Kristallstruktur gewonnen werden.

Aufbau Für die Messungen der Raman-Spektroskopie wird ein LabRam HR Evolution Raman-Spektrometer des Herstellers Horiba verwendet [149]. Eine vereinfachte schematische Darstellung des Aufbaues ist in Abbildung 4.4 zu sehen. Die Raman-Spektren werden unter Verwendung eines Nd:YAG- 
Lasers mit einer Wellenlänge von $\lambda=532 \mathrm{~nm}$ in unterschiedlichen Polarisationskonfigurationen durchgeführt:

1. der parallelen XX-Konfiguration, bei der der Analysator vor dem Detektor parallel zur einfallenden Laserpolarisation eingestellt ist

2. und in der gekreuzten XY-Konfiguration, bei der der Analysator vor dem Detektor entsprechend senkrecht zur einfallenden Laserpolarisation eingestellt ist.

Die lineare Polarisation des Laserstrahles wird mit einem $\lambda / 2$-Plättchen eingestellt und vor dem Detektor selektiert ein Analysatorkristall zwischen den beiden Polarisationsrichtungen. Aufgrund der hohen Intensität der Raleigh gestreuten Strahlung, wird ein Kantenfilter vor dem Spektroskop eingesetzt. Dieser verhindert eine Überschattung des Ramansignales, limitiert jedoch auch die minimale detektierbare Raman-Verschiebung auf $\approx 100 \mathrm{~cm}^{-1}$ [149]. Die technischen Realisierung des Spektroskops ermöglicht nur die Beobachtung von Stokes-Prozessen.

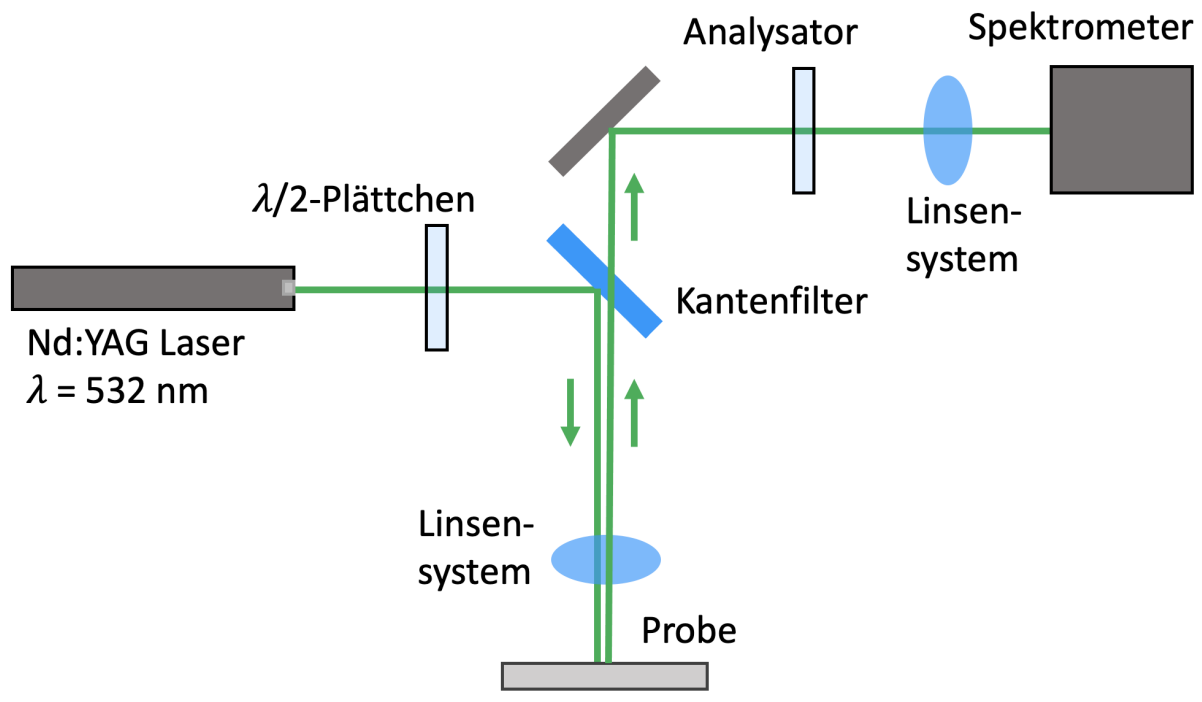

Abbildung 4.4.: Vereinfachte schematische Darstellung des in der Arbeit verwendeten Raman-Aufbaues. 


\subsection{Magnetometrie}

Die Messungen der Magnetisierung der Proben werden mit einem kommerziellen SQUID-Magnetometer (engl.: superconducting quantum interference device) des Typs MPMS (engl.: magnetic property measurement system) XL der Firma Quantum Design durchgeführt. Das Gerät ermöglicht Messungen in einem Temperaturbereich von $2 K$ bis $400 K$ bei kontrollierten Magnetfeldern von bis zu $B_{\max }=5 \mathrm{~T}$. In dem SQUID werden die Proben mit einer periodischen Bewegung durch ein Gradiometer zweiter Ordnung gefahren. Dabei handelt es sich um eine supraleitende Pickup-Spule aus vier Windungen, bei der die zwei mittleren Wicklungen den beiden äußeren gegenüber entgegengesetzt verlaufen. Diese ist induktiv mit einer supraleitenden rf-Quanteninterferenzeinheit (rf-SQUID) verbunden, welche über den Josephson-Effekt und die Flussquantisierung Änderungen des magnetischen Flusses messen kann. Durch die oszillierende Bewegung der Probe mit der Magnetisierung $M$ kommt es zu einer Änderung des magnetischen Flusses $\Phi$, wodurch es zu einer Spannungsinduktion kommt $U_{\text {ind }} \propto \dot{\Phi}$, aus dem sich das magnetische Moment der Probe herleiten lässt. Dies wird in [150,151] näher beschrieben.

Die Proben werden senkrecht zur langen Probenkante mit Hilfe einer Hartgelatine-Kapsel in einem Kunststoffstrohhalm am Probenhalter befestigt und befinden sich somit mit ihrer Schichtoberfläche parallel zum Magnetfeld. Dadurch ergibt sich für Schichten auf einem (100)-Substrat eine Magnetfeldorientierung in [001]-Richtung und auf einem (111)-Substrat eine Feldorientierung in [11-2]-Richtung. Es liegt in der Natur der Dünnfilme, dass neben dem gewünschten Materialsystem auch das Substrat mit gemessen wird. Zusätzlich liefern die Kapsel und der Strohhalm einen schwach diamagnetischen Beitrag. Deshalb muss für die reine Probenmagnetisierung der Anteil vom Substrat und dem Probenhalter abgezogen werden, indem Leermessungen mit einem Substrat unter gleichen Bedingungen durchgeführt werden.

Um die Magnetisierung pro Einheitszelle oder Übergangsmetallion zu bestimmen, benötigt man die Größe der Einheitszelle $V_{\text {u.c. }}$ und das Schichtvo- 
lumen der Probe $V_{\text {Sample }}$. Da die Schichten epitaktisch auf dem Substrat aufgewachsen sind, nimmt die Einheitszelle die in-plane Gitterkonstante des Substrates an, $c_{i n}=c_{S u b}$. Die out-of-plane Gitterkonstante $c_{\text {out }}$ wird aus der XRD-Messung bestimmt. Die Fläche der Probe ist durch die Substratgeometrie und die Abdecksubstrate im MAD-Wachstum vorgegeben $(\approx 0,8 \mathrm{~cm} \cdot 0,5 \mathrm{~cm})^{3}$. Die Schichtdicke $d$ wird über die XRR-Messung bestimmt. Folglich ergibt sich für die Umrechnung des magnetischen Momentes $(e m u)$ in die Magnetisierung der Probe $\left(\mu_{B} /\right.$ u.c. $)$ die Rechnung:

$$
M\left(\mu_{B} / \text { u.c. }\right)=m(e m u) \cdot \frac{V_{u . c .}}{V_{\text {Sample }}} \cdot \frac{1}{\mu_{B}}
$$

Möchte man die Magnetisierung in Abhängigkeit der Temperatur messen, ergeben sich unterschiedliche Möglichkeiten dies durchzuführen. In der vorliegenden Arbeit werden folgende Vorgehen angewandt:

1. Messungen beim Abkühlen im Magnetfeld, $H=100$ Oe bzw. 1000 Oe (FCC, engl.: field-cooled cooling).

2. Messungen beim Aufwärmen im Magnetfeld nach vorherigen Erwärmung ohne Magnetfeld (ZFC, engl.: zero-field cooling).

Aus der temperaturabhängigen Magnetisierungskurve (FCC) wird die CurieTemperatur $T_{C}$ einer ferromagnetischen Probe über das Minimum der logarithmischen Ableitung der Magnetisierungskurve bestimmt:

$$
T_{C}=\min \left(\frac{1}{M(T)} \cdot \frac{d M(T)}{d T}\right)
$$

\footnotetext{
${ }^{3}$ Die durch die Abdecksubstrate verdeckte Fläche muss für jede Probe individuell mit einem digitalen Messschieber bestimmt werden. Erfahrungswerte zeigen, dass sich im Mittel eine Abdeckung von $1 \mathrm{~mm}$ pro Abdecksubstrat ergibt.
} 


\subsection{Messung des elektrischen Widerstandes}

Die elektrischen Transporteigenschaften der Dünnfilme werden mit einem PPMS-Gerät (engl.:physical property measurement system) der Firma Quantum Design gemessen [152]. Dabei handelt es sich um ein multifunktionales System, das in einen He-Kryostaten eingebaut ist und verschiedene physikalische Größen temperatur- und magnetfeldabhängig messen kann. Mit Hilfe eines supraleitenden Magnetes können in dem Kryostaten kontrolliert Magnetfelder bis zu $B_{\max }=9 T$ in der Probenkammer angelegt werden und die Temperatur in einem Bereich $T=1,9 K-400 K$ variiert werden. Das PPMS wird zur Messung des Gleichstomwiderstandes verwendet. Um den Einfluss von Leitungs- und Kontaktwiderständen gering zu halten, wird der Widerstand der Probe mit der Vier-Punkt-Methode gemessen, wie in Abbildung 4.5 a) zu sehen ist. Dafür wird die Probe auf einen speziellen Probenhalter, der in Abbildung 4.5 b) abgebildet wird, befestigt und mit dünnen Kupferdrähten und Leitsilber kontaktiert. Durch die Vorgabe des Stroms über die äußeren Kontakte und die Messung der an der Probe abfallenden Spannung über die inneren Kontakte, kann der elektrische Widerstand der Probe mit sehr hoher Genauigkeit über das ohmsche Gesetz bestimmt werden. Für die Auswertung an den dünnen Filmen wird im Rahmen der vorliegenden Arbeit der spezifische Flächenwiderstand $\rho_{F}=R \cdot d$ verwendet ( $d$ Schichdicke und $R$ der gemessene Widerstand).

a)

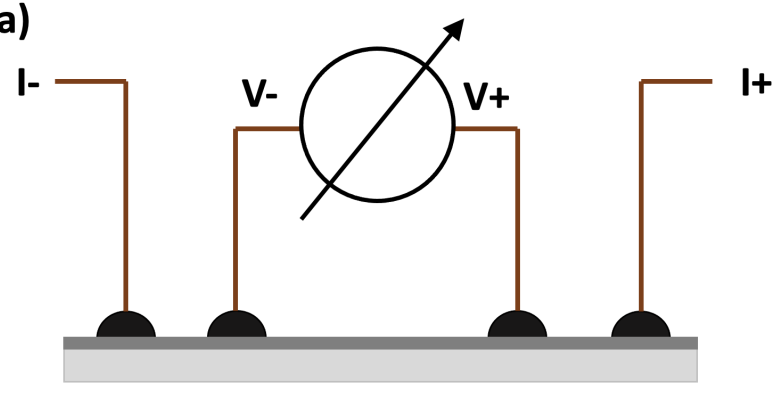

b)

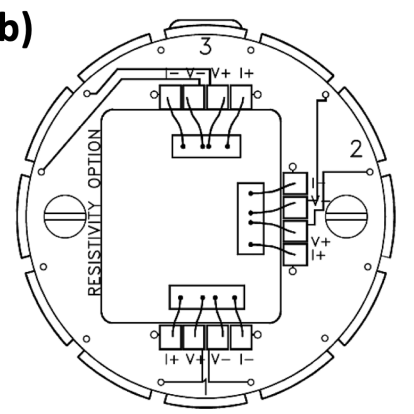

Abbildung 4.5.: a) Schematische Darstellung der Messung des Gleichstromwiderstandes mit der Vier-Punkt-Methode, b) PPMSProbenhalter zum Einbau in das PPMS. Der verwendete Probenhalter erlaubt es drei Proben zeitgleich kontaktiert zu haben und zu vermessen. b) entnommen aus [152]. 
Hall-Messungen Schlussendlich werden in Zusammenarbeit mit der Arbeitsgruppe um Philipp Gegenwart an der Universität Augsburg Hall-Messungen zur Untersuchung der anomalen und topologischen Halleffektes angefertigt [153]. Dafür muss aus einer angefertigten Probe mit der MAD in einem ersten Schritt eine geeignete Struktur, ein sogenannter Hall-Barren, hergestellt werden. Ähnlich wie zur Herstellung der Lamelle für das TEM, wird dabei das FIB Crossbeam 550 von der Firma Zeiss [154] verwendet. Die Struktur ist so konstruiert, dass die Spannung parallel und senkrecht zur Stromrichtung gemessen werden kann, wie in Abbildung $4.6 \mathrm{zu}$ sehen ist. Aus der Spannung parallel zum Strom $U_{M R}$ lässt sich der Magnetowiderstand (MR, engl.: magnetoresistance) messen und aus der Spannung senkrecht zum Strom die Hall Spannung $U_{\text {Hall }}$.

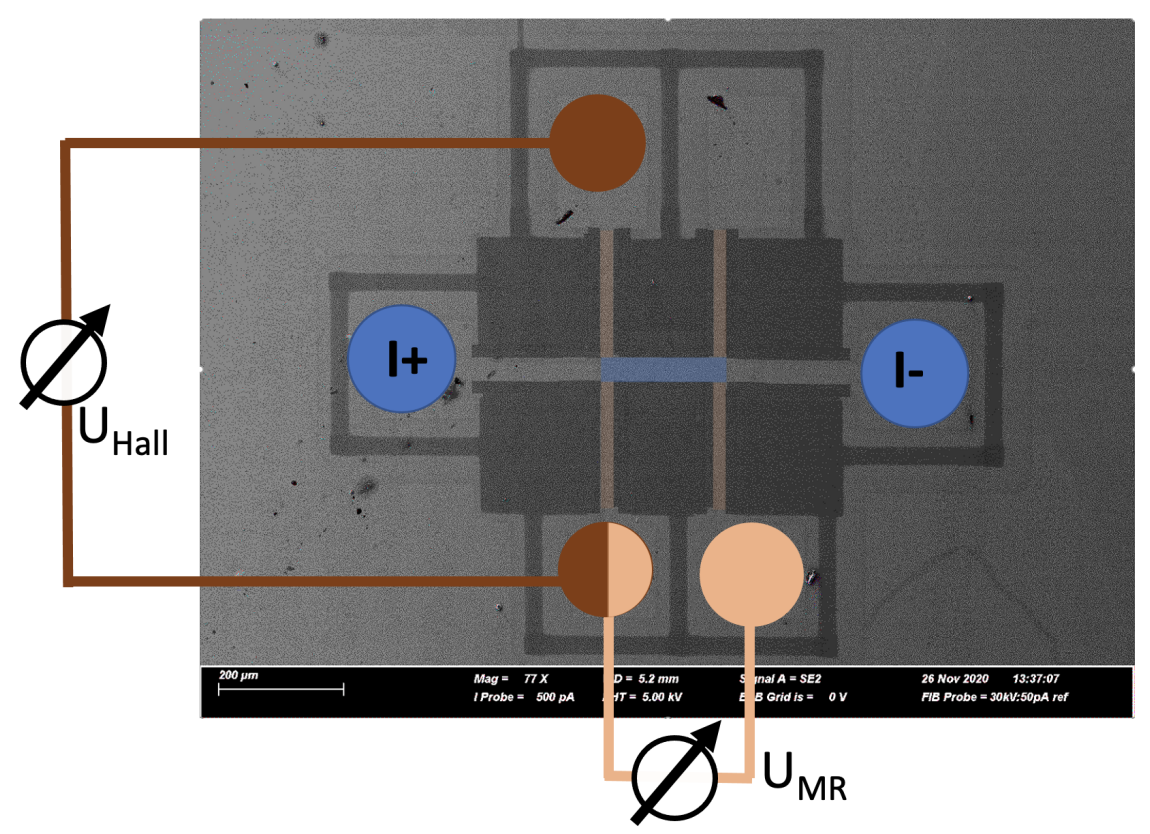

Abbildung 4.6.: Aufnahme eines Elektronenstrahlmikroskopes einer HallBar Struktur (( $\left.\mathrm{SrIrO}_{3} / \mathrm{SrMnO}_{3}\right)$ auf $\mathrm{SrTiO}_{3}$ in (111)Orientierung) mit eingezeichnetem Kontaktierungsschema. Die helle Fläche zeigt die intakte Probe, während die dunkle Fläche das Substrat ist, das mittels FIB freigelegt wird. Angefertigt von Robert Gruhl an der Universität Augsburg und entnommen aus [153]. 
Die Probe wird nach der Bearbeitung in einem bereits vorgestellten PPMS vermessen. Der Probenaufsatz ist dabei so konstruiert, dass die Probe um die Stromachse rotiert werden kann und so winkelabhängige Messungen ermöglicht (von $-10^{\circ}$ bis $360^{\circ}$ ). Ein angelegtes Magnetfeld steht dabei immer senkrecht zum Strom, der über eine Wechselspannung mit $128 \mathrm{~Hz}$ erzeugt wird. Bei einer Einstellung von $0^{\circ}$ steht das Magnetfeld senkrecht zur Probenoberfläche, sodass der normale Halleffekt bei diesem Winkel am stärksten ausgeprägt sein sollte. Bei den Untersuchungen des Halleffektes wird das Magnetfeld kontinuierlich variiert [153]:

$$
T \rightarrow 5 T \rightarrow-5 T \rightarrow 5 T \rightarrow-5 T \rightarrow 0 T \text {. }
$$

Die Messung des Halleffektes wird durch den Magnetowiderstand beeinflusst und überlagert. Aus diesem Grund müssen beide Signale voneinander getrennt werden. Da der Magnetowiderstand einen symmetrischen Verlauf zum Ursprung hat $\left(R_{M R}(B)=R_{M R}(-B)\right)$ und der Hallwiderstand asymmetrisch ist $\left(R_{\text {Hall }}(B)=-R_{\text {Hall }}(-B)\right)$, lassen sich beide Effekte numerisch aus dem Signal ermitteln. 


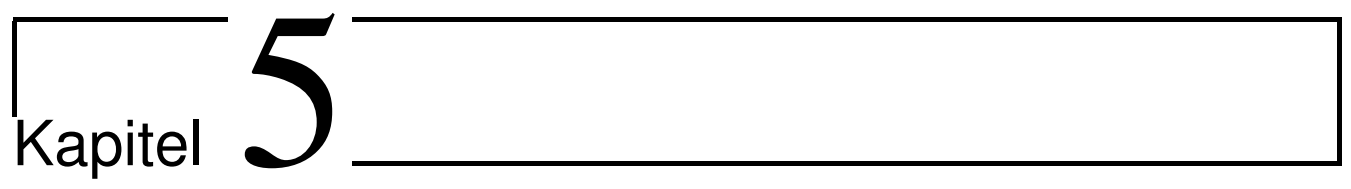

\section{Ergebnisse und Diskussion}

Motiviert durch den Anspruch vollständig geordnete Doppelperowskite herzustellen, widmet sich der erste Teil der vorliegenden Arbeit der Fragestellung, wie die Wachstumsbedingungen der MAD die treibenden Kräfte der B-Platzordnung beeinflussen und wie diese genutzt bzw. ausgebaut werden können, um den bisherigen Grad der Ordnung weiter zu erhöhen. Aus diesem Ziel heraus entstand das Konzept des alternierenden Aufwachsen der einzelnen Atomlagen, um so die B-Platzordnung künstlich zu erzeugen. Dieses Verfahren der ,Synthese künstlich erzwungener geordneter Doppelperowskite" (LbL, engl.; Layer by Layer growth mode) wird zunächst auf das Materialsystem aus $\mathrm{LaNiO}_{3}$ und $\mathrm{LaMnO}_{3}$ angewandt. Um jedoch genau eine Monolage kontrolliert zu wachsen, benötigt es ein hohes Maß an Genauigkeit der zu deponierenden Lösungsmenge pro Einheitszelle. Aus diesem Grund wird an diesem System der Ansatz gewählt, sich dem Monolagenwachstum zu nähern, indem zunächst Heterostrukturen $\left(\mathrm{LaNiO}_{3}\right)_{n} /\left(\mathrm{LaMnO}_{3}\right)_{n}$ mit $\mathrm{n}=20,10,5,2$ und schließlich 1 hergestellt werden, um möglichst genau die zu deponierende Lösungsmenge zu ermitteln. Die stetige Reduzierung der Einzellagendicke ermöglicht dabei einen tiefen Einblick in die Grenzflächenphysik zwischen $\mathrm{LaNiO}_{3}$ und $\mathrm{LaMnO}_{3}$ und den Übergang zum gängigen (SoA, engl.; State of the Art growth mode) $\mathrm{La}_{2} \mathrm{NiMnO}_{6}$. Die Ergebnisse der Grenzflächeneffekte und der auftretenden Doppelperowskitphase werden in den ersten Abschnitt präsentiert, bevor die Kationordnung zwischen Lagenwachstum und SoA-Wachstum verglichen werden. 
Anschließend werden die gewonnenen Erfahrungen auf weitere Doppelperowskite angewandt: $\mathrm{La}_{2} \mathrm{CoMnO}_{6}$ und $\mathrm{Sr}_{2} \mathrm{TiRuO}_{6}$. Jedoch wird hierbei die kontrollierte B-Platzordnung auf eine andere Weise realisiert: anstelle die Einzellagendicke in Schritten zu reduzieren wird hier die B-Platzordnung durch systematische Variation der Lagenstöchiometrie erzielt, indem das Verhältnis der Depositionsmengen der $V_{\mathrm{ABO}_{3}} / V_{\mathrm{AB}^{\prime} \mathrm{O}_{3}}$ kontrolliert angepasst wird. Auf diese Weise ergibt sich eine weitere Methode, die B-Platzordnung in Doppelperowskiten künstlich zu erzeugen, und diese zeigt, welchen Einfluss die lagenweise Stöchiometrie in DP-Dünnfilmen auf das System hat. Die gewonnenen Erkenntnisse dieses Verfahrens werden vorgestellt und der so erreichte Ordnungsgrad wird außerdem mit der bisher gängigen SoA-Methode verglichen.

Den Abschluss dieser Arbeit bildet die Erweiterung auf Heterostrukturen mit dem 5d-System $\mathrm{SrIrO}_{3}$. Dabei wird der Ansatz der kontinuierlichen Reduzierung der Einzellagendicke, wie in $\left(\mathrm{LaNiO}_{3}\right)_{n} /\left(\mathrm{LaMnO}_{3}\right)_{n}$, auf das Materialsystem $\left(\mathrm{SrIrO}_{3}\right)_{n} /\left(\mathrm{SrMnO}_{3}\right)_{n}$ mit $\mathrm{n}=5,2$ und 1 angewandt. Anders als zuvor werden die Proben auf STO in (100)-Orientierung hergestellt. Um die resultierende B-Platzordnung der LbL-Probe genauer zu untersuchen, wird diese mit SoA-Referenzprobenlauf STO mit (111)- und (100)-Orientierung verglichen. In Zusammenarbeit mit der Arbeitsgruppe um Philipp Gegenwart werden an der Universität Augsburg Hallmessungen zur Untersuchung des anomalen und topologischen Halleffektes an dem System durchgeführt. Die Suche nach dem anomalen und topologischen Halleffekt wird schließlich auf Heterostrukturen aus $\left(\mathrm{La}_{0.7} \mathrm{Sr}_{0.3} \mathrm{MnO}_{3}\right)_{n} /\left(\mathrm{SrIrO}_{3}\right)_{2}$ mit $\mathrm{n}=6$ und 4 erweitert. Dabei werden die Grenzflächeneffekte abschließend untersucht, die die Grundlage für zukünftige Forschungsunternehmungen bilden. 


\subsection{Grenzflächeneffekte, emergent Doppelperowskitphase und Kationordnung in}

$\left(\mathrm{LaNiO}_{3}\right)_{n} /\left(\mathrm{LaMnO}_{3}\right)_{n}$-Heterostrukturen

Rechtliches und Lizenzen Das folgende Kapitel fasst einen weiten Teil der wissenschaftlichen Ergebnisse der vorliegenden Promotion zusammen. Große Teile davon wurden bereits in den Publikationen „Emergent Double Perovskite Phase at $\mathrm{LaMnO}_{3} / \mathrm{LaNiO}_{3}$ Interfaces: Coupled Charge Transfer and Structural Reconstruction" [155] (Copyright 2021 by the American Physical Society) und „B-site Cation Ordering in Films, Superlattices and Layerby-Layer Grown Double Perovskites" [63] veröffentlicht, in denen ich als Erstautor angegeben bin und den Hauptanteil der Ergebnisse produziert habe. Die Erlaubnis Abbildungen, Tabellen und inhaltliche Formulierungen aus dem Englischen zu übersetzten und für die vorliegende Dissertation zu verwenden, wurde von der American Physical Society eingeholt. Abbildungen, Tabellen und inhaltliche Formulierungen, die unter der Creative Common CC BY Lizenz veröffentlicht wurden, werden entsprechend zitiert. Messungen und Abbildungen, die nicht direkt von mir stammen, werden als solche mit einem Verweis gekennzeichnet.

\subsubsection{Grenzflächeneffekte zwischen $\mathrm{LaNiO}_{3}$ und $\mathrm{LaMnO}_{3}$}

Einzelschichtssysteme $\mathrm{LaMnO}_{3}$ und $\mathrm{LaNiO}_{3}$ Noch bevor die Heterostrukturen hergestellt werden können, ist es unerlässlich die Wachstumsbedingungen und Eigenschaften der Ausgangssysteme $\mathrm{LaNiO}_{3}$ und $\mathrm{LaMnO}_{3}$ separat zu untersuchen und zu optimieren. Sämtliche Proben sind auf kommerziellen $\mathrm{SrTiO}_{3}$-Substraten der Firma Crystal [156] in (111)-Orientierung bei einer Depositionstemperatur von $T_{D e p}=900{ }^{\circ} \mathrm{C}$ hergestellt. Die Depositionsgeschwindigkeit beträgt $r=0,04 \mathrm{ml} / \mathrm{s}$, woraus sich für die Einzelsysteme aus Messungen der Schichtdicken eine Wachstumsrate von $v_{L M O}=$ 0,22 u.c./s für $\mathrm{LaMnO}_{3}$ und $v_{L N O}=0,18$ u.c./s für $\mathrm{LaNiO}_{3}$ ergibt. 
Optimierungen der elektrischen, magnetischen und morphologischen Eigenschaften (Abbildung 5.1) ergeben für die Dünnfilme aus $\mathrm{LaMnO}_{3}$ ein optimales molares Verhältnis von $L a / M n=1,45$ in der Depositionslösung. Während $\mathrm{LaMnO}_{3}$ in seiner stöchiometrischen Bulkform ein isolierender A-Typ-Antiferromagnt mit einer Néel-Temperatur von $T_{N}=140 \mathrm{~K}$ ist [99,157], zeigt es als Dünnfilm für gewöhnlich isolierendes ferromagnetisches Verhalten, wie bereits in Kapitel 2.3.1 erläutert [49,158]. Die epitaktische Referenzschicht auf STO(111) mit einer Dicke von $d=15,9 \mathrm{~nm}$ weist ebenfalls isolierendes ferromagnetisches Verhalten auf. Die Curie-Temperatur be-
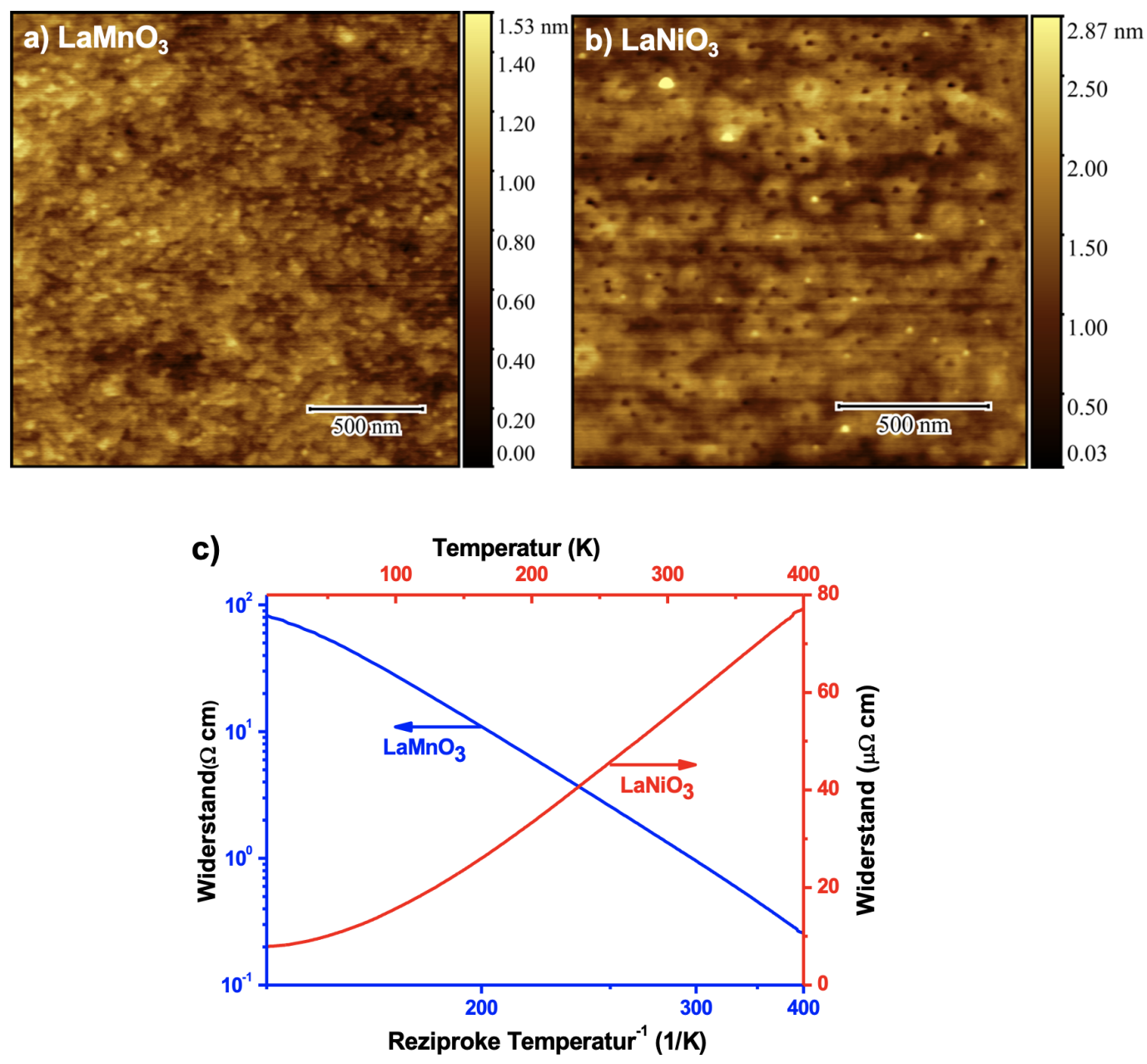

Abbildung 5.1.: AFM-Aufnahme der separaten Einzelschichten a) $\mathrm{LaMnO}_{3}$, b) $\mathrm{LaNiO}_{3}$ auf $\mathrm{STO}(111)$ und c) deren spezifischen Flächenwiderstände. Mit Erlaubnis entnommen aus [155]. 
trägt $T_{C}=158 \mathrm{~K}$ und die Sättigungsmagnetisierung erreicht $M_{\text {sat }}(5 K)=$ $2,8 \mu_{B} / M n$, wie in der Abbildung 5.9 a) zu sehen ist. $\mathrm{LaMnO}_{3}$ weist als Bulk orthorhombische Pbnm-Kristallstruktur auf $[159,160]$. Da die XRDMessungen keine Strukturpeaks der Schicht zeigen, wird angenommen, dass die Gitterkonstante $c_{L M O}$ ungefähr der Gitterkonstante von $\mathrm{SrTiO}_{3}$ entspricht, $c_{L M O} \approx c_{S T O}=0,3905 \mathrm{~nm}$. Abbildung 5.1 a) zeigt die Oberflächenmorphologie der Referenzschicht, die mit einem RMS-Wert von 0,2 $\mathrm{nm}$ sehr glatt ist, was für die Herstellung feiner Grenzflächen in den Heterostrukturen Voraussetzung ist.

Da $\mathrm{LaNiO}_{3}$ ein paramagnetisches Metall ist ${ }^{1}$, wird es auf die elektrischen Eigenschaften und die Oberflächenmorphologie hin optimiert. So ergab sich für die Depositionslösung ein molares Verhältnis von $L a / N i=1,33$. Die Referenzprobe mit einer Schichtdicke von $d_{L N O}=16,8 \mathrm{~nm}$ zeigt einen geringen Restwiderstand von $\rho(5 \mathrm{~K})=8 \cdot 10^{-6} \Omega \mathrm{cm}$ mit einem Restwiderstandsverhältnis von $R R R=R(300 K) / R(5 K) \approx 7$, wie in Abbildung $5.1 \mathrm{c}) \mathrm{zu}$ entnehmen ist. XRD-Messungen der out-of-plane Gitterkonstante ergeben gegenüber den Literaturwerten der Bulkproben einen signifikant kleineren Wert, $c_{L N O}=0,3767 \mathrm{~nm}<c_{L N O, B u l k}=0,384 \mathrm{~nm}$ [161], was auf das Vorhandensein einer großen Zugspannung $\epsilon=-2 \%$ auf dem $\mathrm{SrTiO}_{3}$-Substrat zurückzuführen ist. Das System kristallisiert in der rhomboedrischen $R-3 c$ Struktur $[164,165]$. Auch hier bestätigt ein geringer RMS-Wert von 0,3 nm die hohe Schichtqualität, wie in siehe Abbildung 5.1 b) zu sehen ist.

Wachstum und Struktureigenschaften der Heterostrukturen Unter den ermittelten Wachstumsbedingungen werden die Übergitter $\left(\mathrm{LaMnO}_{3}\right)_{n} /$ $\left(\mathrm{LaNiO}_{3}\right)_{n}$ mit $n=20,10,5,2$ und 1 u.c. (n/n SL) und einer Schichtdicke von $d=22 \mathrm{~nm}$ (d.h. 100 u.c. für die Ellipsometrie-Auswertung) und 44 nm (d.h. 200 u.c. für die Auswertung der magnetischen Eigenschaften) durch kontrollierte und sequentielle Deposition der La(acac) ${ }_{3} \mathrm{Mn}(\mathrm{acac})$ und $\mathrm{La}(\mathrm{acac})_{3} \mathrm{Ni}(\mathrm{acac})-$ Lösungen auf $\mathrm{STO}(111)$ gewachsen, beginnend mit $\mathrm{LaNiO}_{3}$. Die Probenserie wird mit dem 20/20 SL gestartet und stetig bis zum 1/1 SL reduziert, um eine präzise Einzelschichtdicke zu erhalten.

\footnotetext{
${ }^{1}$ Diese Eigenschaften zeigt es als einziges Mitglied der Nickelfamilie $\mathrm{ReNiO}_{3}$ (mit $\mathrm{Re}=$ seltene Erden) [161-163].
} 


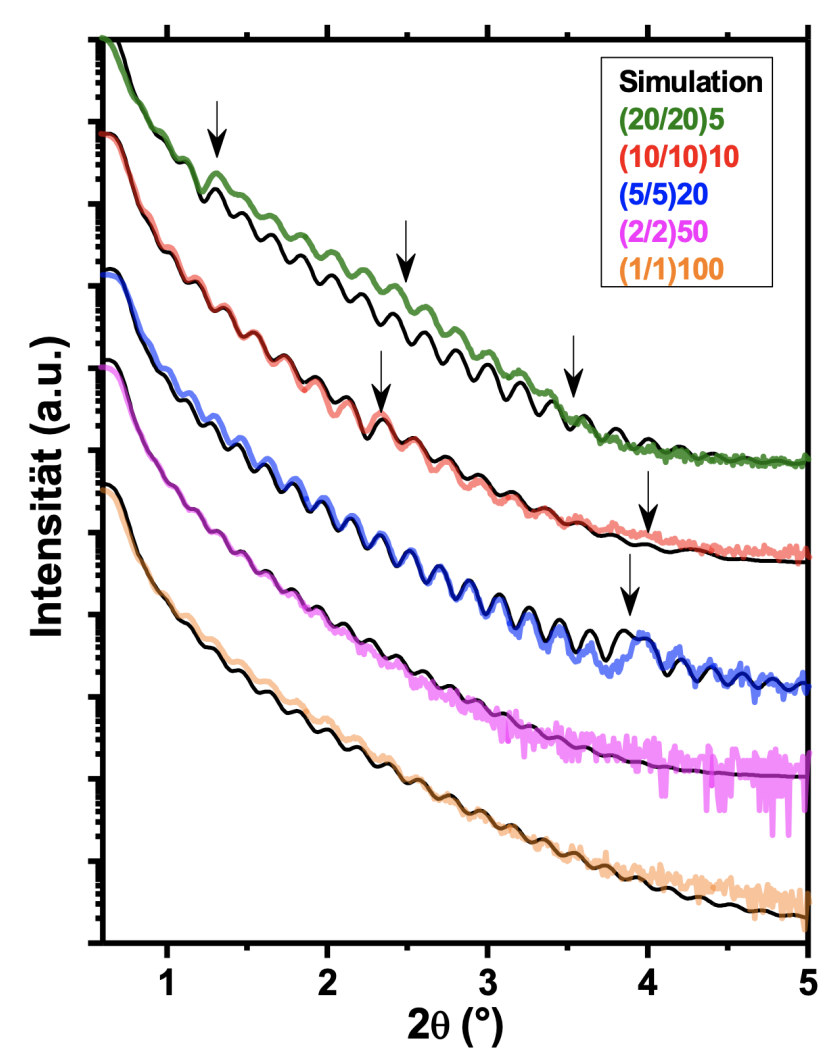

Abbildung 5.2.: XRR-Messungen der Heterostrukturen (20/20, Grün), (10/10, Rot), (5/5, Blau), (2/2 Magenta) und schließlich (1/1, Orange) mit jeweiliger Simulation nach dem ParrattFormalismus (Schwarz). Für die (20/20), (10/10) und (5/5) Proben sind deutlich die Überstrukturpeaks zu sehen, die durch die Periodizität der $\mathrm{LaNiO}_{3} / \mathrm{LaMnO}_{3}$-Schichten entstehen. Mit Erlaubnis entnommen aus [155].

Die Gesamtschichtdicke, die Dicke der Einzelschichten und die Grenzflächenrauigkeiten werden über XRR-Messungen in Verbindung mit Simulationen über das GenX-Programm [143] unter Verwendung des Parratt-Formalismus ermittelt und in Tabelle 5.1 zusammengefasst. Die XRR-Messungen mit den Simulationen sind aus Abbildung $5.2 \mathrm{zu}$ entnehmen. Die Simulationen ergeben zunächst, dass die Grenzflächen zwischen den einzelnen Schichten mit einem RMS-Wert von ca. 0,2 $\mathrm{nm}$ sehr glatt sind. Dies entspricht ungefähr einer Gitterkonstante entlang der (111)-Richtung. Allerdings ergibt sich durch genaueres Betrachten der Simulationen, dass das Messsignal und die einhergehende Grenzflächenrauigkeit empfindlich von den Rauigkeitswerten der zwei oberflächennahen Schichten (RMS Top in Tabelle 5.1) abhängt und 
Tabelle 5.1.: Ergebnisse der strukturellen Untersuchung mittels XRRMessung in Verbindung mit der Simulation der Übergitter $(n / n)$, der SoA $\mathrm{La}_{2} \mathrm{NiMnO}_{6}$-Probe und der Einzelschichtsysteme $\mathrm{LaNiO}_{3}$ und $\mathrm{LaMnO}_{3}$. Die Gitterkonstante der Schichten $c_{a v}$ ergibt sich aus den XRD-Messungen, die im Anhang A.3 zu finden sind.

\begin{tabular}{c|ccccc}
\hline \hline & $\begin{array}{c}\text { Schicht- } \\
\text { dicke } \\
d[\mathrm{~nm}]\end{array}$ & $\Delta d$ & $\begin{array}{c}\text { Gitter- } \\
\text { konstante } \\
c_{a v}[\mathrm{~nm}]\end{array}$ & $\begin{array}{c}\text { RMS } \\
\text { Grenzfläche } \\
{[\mathrm{nm}]}\end{array}$ & $\begin{array}{c}\text { RMS } \\
\text { Top } \\
{[\mathrm{nm}]}\end{array}$ \\
\hline $20 / 20$ & 43,36 & $-2,2$ & 0,3810 & 0,21 & 0,56 \\
$10 / 10$ & 41,56 & $-6,6$ & 0,3815 & 0,22 & 0,63 \\
$5 / 5$ & 45,8 & 3,2 & 0,3812 & 0,21 & 0,62 \\
$2 / 2$ & 50,75 & 12,7 & 0,3808 & 0,1 & 0,52 \\
$1 / 1$ & 44,1 & $-0,05$ & 0,3808 & 0,1 & 0,52 \\
\hline $\mathrm{SoA}_{\mathrm{LaNiO}}$ & 79,7 & - & 0,3810 & - & - \\
$\mathrm{LaMnO}_{3}$ & 16,8 & - & 0,3767 & - & - \\
\hline \hline
\end{tabular}

nahezu unabhängig von der Rauigkeit der darunter liegenden Grenzflächen ist. Dies macht es schwierig die genaue Rauigkeit innerhalb des Übergitters mithilfe der Röntgenmethoden zu bestimmen. Für alle Proben ergab sich die beste Übereinstimmung zwischen Messung und Simulation, in dem höhere RMS-Werte für die beiden abschließenden zwei Schichten verwendet werden, die sich im Bereich von 0,5 $\mathrm{nm}$ bis 0,6 $\mathrm{nm}$ befinden.

Weil auf diese Weise nur schwierig aussagekräftige Rückschlüsse möglich sind, werden TEM-Untersuchungen an der (5/5) Probe durchgeführt, um einen tieferen Einblick in die Grenzflächenbeschaffenheit innerhalb der Probe zu erlangen, die in Abbildung 5.3 dargestellt sind. Die Abfolge der einzelnen Einheitszellen, die in Grün $\left(\mathrm{LaMnO}_{3}\right)$ und Orange $\left(\mathrm{LaNiO}_{3}\right)$ hervorgehoben sind, bestätigt die Periodizität und Schichtdicke der Einzelschichten der Probe. Der Verlauf der Grenzflächen $\mathrm{LaMnO}_{3}$ (oben)/ $\mathrm{LaNiO}_{3}$ (unten) $(\mathrm{NM})$ und andersrum (MN) ist durch blaue und gelbe Linien in Abbildung 5.3 markiert. Der angedeutete Verlauf deutet auf strukturell asymmetrische Grenzflächen hin, bei der die NM-Grenzfläche glatt und die MN-Grenzfläche vergleichsweise rauer ist. Dieses Ergebnis sollte jedoch mit Vorsicht behandelt werden, da die Auflösung der Grenzflächen aufgrund des geringen Kon- 
trastes zwischen Mn und Ni nicht ausreichend ist.

Die ermittelten Gesamtschichtdicken der Proben passen gut zu dem theoretischen Wert von $d=44,31 \mathrm{~nm}$ für bei 200 Einheitszellen, wie in Tabelle 5.1 durch $\Delta d / d$ dargestellt. Eine einzige Ausnahme bildet dabei das $(2 / 2)$ Übergitter, das mit $12 \%$ deutlich von dem zu erwarteten Wert abweicht, woraus sich eine mittlere Einzelschichtdicke von 2,3 u.c. ergibt. Diese deutliche Abweichung kann durch die veränderte Wachstumskinetik und/oder die Änderung der Gitterkonstanten beim Übergang von 5 u.c. auf 2 u.c. verursacht werden, da die $(2 / 2)$ Heterostruktur einen elektronischen und strukturellen Übergang zur DP $\mathrm{La}_{2} \mathrm{NiMnO}_{6}$-Phase zeigt, wie im Folgenden noch diskutiert wird [155].

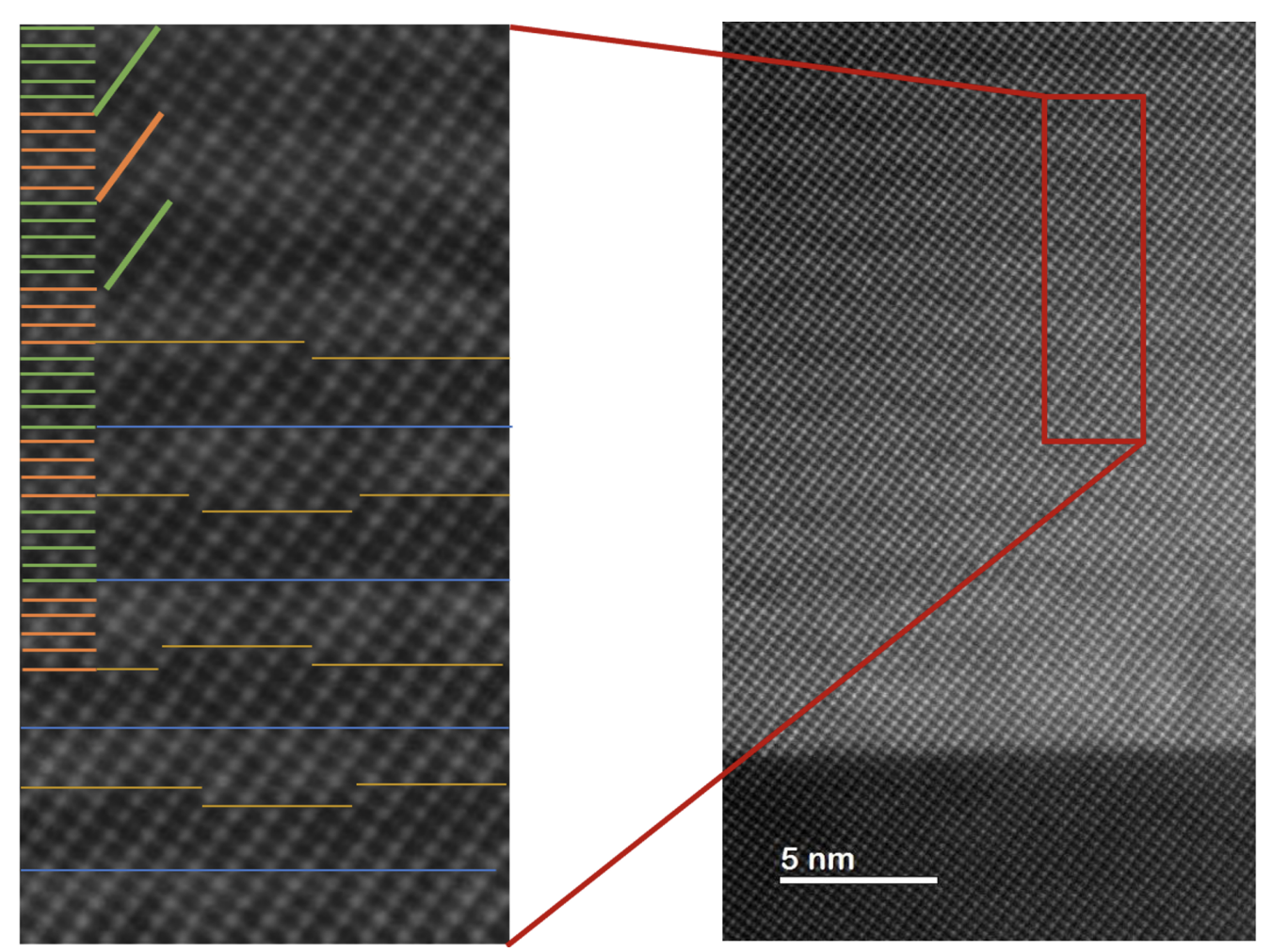

Abbildung 5.3.: HAADF STEM-Messung der (5/5) Probe mit Vergrößerung. Die einzelnen Einheitszellen der $\mathrm{LaNiO}_{3}$-Schicht sind Orange und die der $\mathrm{LaMnO}_{3}$-Schicht Grün hervorgehoben. Zusätzlich sind in Blau und Gelb die eingeschätzten Grenzflächenverläufe eingezeichnet. Das Bild wurde von Vladimir Roddatis in der Universität Göttingen angefertigt und mit Erlaubnis entnommen aus [155]. 
Ellipsometrie Messungen Die in-situ-Ellipsometrie-Messungen der separaten Einzelschichten $\mathrm{LaMnO}_{3}$ und $\mathrm{LaNiO}_{3}$ sind in Abbildung 5.4 dargestellt. Beide zeigen einen ähnlichen Verlauf, in dem sich nach fünf bis zehn gewachsenen Einheitszellen die Phasenänderung $A=d \Delta / d D$ (mit $d=r \cdot t$, wobei $D$ bei einer konstanten Wachstumsrate $r$ der Schichtdicke entspricht) einen nahezu konstanten Verlauf annimmt [155]. Für die beiden Materialsysteme ergeben sich dabei unterschiedliche Werte für die Phasenänderung, $A_{L M O} \approx 0,23^{\circ} /$ u.c. $\ll A_{L N O, \text { avg }} \approx 1,2^{\circ} /$ u.c., die auf das metallische Verhalten im $\mathrm{LaNiO}_{3}$ und das isolierende Verhalten in $\mathrm{LaMnO}_{3}$ und der damit verbundenen Elektronendichte zurückzuführen sind. Simulationen der Ellipsometriegrößen $\Psi(t)$ und $\Delta(t)$ ergeben für $\mathrm{LaMnO}_{3}$ einen komplexen Brechungsindex von $N_{L M O}=2,29-i \cdot 0,27$, welcher mit vorherigen Angaben in der Literatur übereinstimmt $[124,166]$. Der ermittelte Brechnungsindex für $\mathrm{LaNiO}_{3}$ beträgt $N_{L N O}=2,05-i \cdot 0,81$ und weicht damit leicht von den Literaturwert für $\mathrm{LaNiO}_{3}$ auf $\operatorname{Si}(111)\left(N_{L N O, S i}=2,1-i \cdot 0,9\right)$, das mit der PLD gewachsen wird, ab [167].
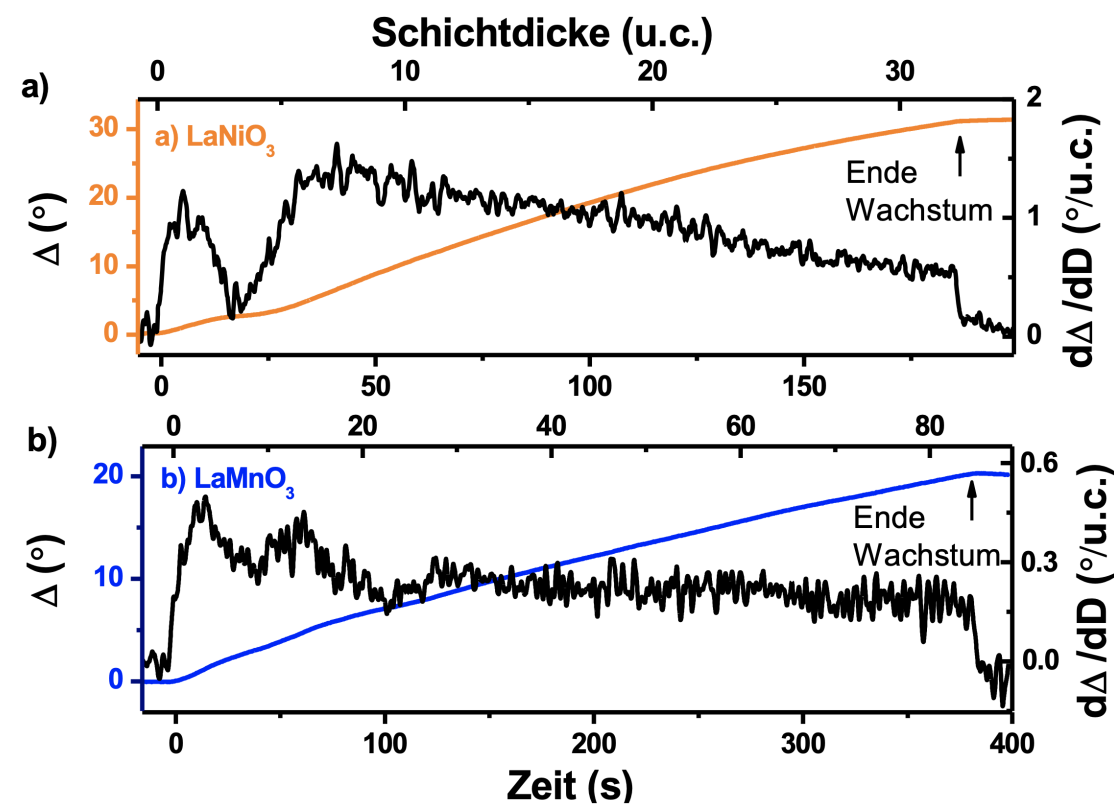

Abbildung 5.4.: Die Abhängigkeit der Phasenwinkels von der Zeit $\Delta(t)$ und der Schichtdicke $\Delta(d)$ der a) $\mathrm{LaNiO}_{3^{-}}\left(d_{L N O}=12,6 \mathrm{~nm}\right)$ und b) $\mathrm{LaMnO}_{3^{-}}\left(d_{L M O}=32,5 \mathrm{~nm}\right)$ Probe auf STO(111). Die ermittelte Änderung der Phasenverschiebung ist ebenfalls dargestellt. Mit Erlaubnis entnommen aus [155]. 
Die gesamte Messung des Phasenwinkels $\Delta(t)$ für die (10/10) und (5/5) Probe während des Wachstums ist in Abbildung 5.5 a) und b) dargestellt. Die orange hinterlegten Bereiche zeigen die Deposition des $\mathrm{LaNiO}_{3}$ und die blau hinterlegten die des $\mathrm{LaMnO}_{3}$. Zwischen den einzelnen Depositionen werden Pausen eingelegt, damit das Wachstum der jeweilige Einzelschicht ungestört abgeschlossen werden kann und Reste des Aerosols abgepumpt werden können. Änderungen des Messsignales, die während der Pause entstehen, werden auf einen induzierten Temperaturanstieg während der Pyrolysereaktion des MAD-Wachstums und eine anschließende Abkühlung zurückgeführt. Das Wachstum beider Proben beginnt mit $\mathrm{LaNiO}_{3}$, sodass das Signal dem Verlauf der $\mathrm{LaNiO}_{3}$-Einzelschicht, wie in Abbildung $5.4 \mathrm{a}$ ), folgt ${ }^{2}$. Entgegen der Erwartungen für die darauf folgende $\mathrm{LaMnO}_{3}$-Schicht und dem in Abbildung 5.4 b) beobachteten Anstieg des Phasenwinkels, sinkt der Phasenwinkel innerhalb der ersten beiden $\mathrm{LaMnO}_{3}$-Schichten. Aufgrund der komplexen mathematischen Beschreibung, die aus der Mehrfachreflexion des Lichtes an den einzelnen Grenzflächen der Heterostruktur resultiert (siehe Kapitel 3.3), ist der Verlauf von $\Delta(t)$,intuitiv" allerdings nicht vorhersehbar. Aus diesem Grund werden Simulationen des zeitlichen Verlaufes des Phasenwinkels nach der Theorie in Kapitel 3.3 für die (10/10) Probe angefertigt, die in Abbildung 5.5 a) zusätzlich dargestellt sind. Die simulierten Punkte in roter Farbe werden unter Verwendung des komplexen Brechungsindex für $\mathrm{LaNiO}_{3}$ und $\mathrm{LaMnO}_{3}$ aus Abbildung 5.4 ermittelt, während in blauer Farbe ein abgeänderter Brechungsindex $N_{L M O}=2,5-i \cdot 0,27$ für $\mathrm{LaMnO}_{3}$ für die Simulation verwendet wird. Es ist deutlich zu erkennen, dass der modifizierte Brechungsindex des $\mathrm{LaMnO}_{3}$ zu einer deutlicheren Übereinstimmung mit dem Messergebnis führt. Diese Beobachtung liefert einen Hinweis darauf, dass die elektronischen Eigenschaften des $\mathrm{LaMnO}_{3}$ durch die darunter liegende $\mathrm{LaNiO}_{3}$-Schicht beeinflusst wird [155]. Diese Beobachtung ist für die Proben mit geringerer Einzelschichtdicke (5/5) (Abbildung 5.5 b)) und (2/2) (Abbildung 5.6 a)) sogar noch stärker ausgeprägt. Hier lässt sich jedoch mit dem vereinfachten Modell, in dem die Einzelschichten nicht mitein-

\footnotetext{
${ }^{2}$ Die beobachteten geringen Abweichungen innerhalb der ersten 5 bzw. 10 Einheitszellen der $\mathrm{LaNiO}_{3}$-Schicht resultieren aus unterschiedlichen Starteinstellung innerhalb der MAD-Anlage und durch unterschiedliche Oberflächenmorphologien der verwendeten Substrate [155].
} 

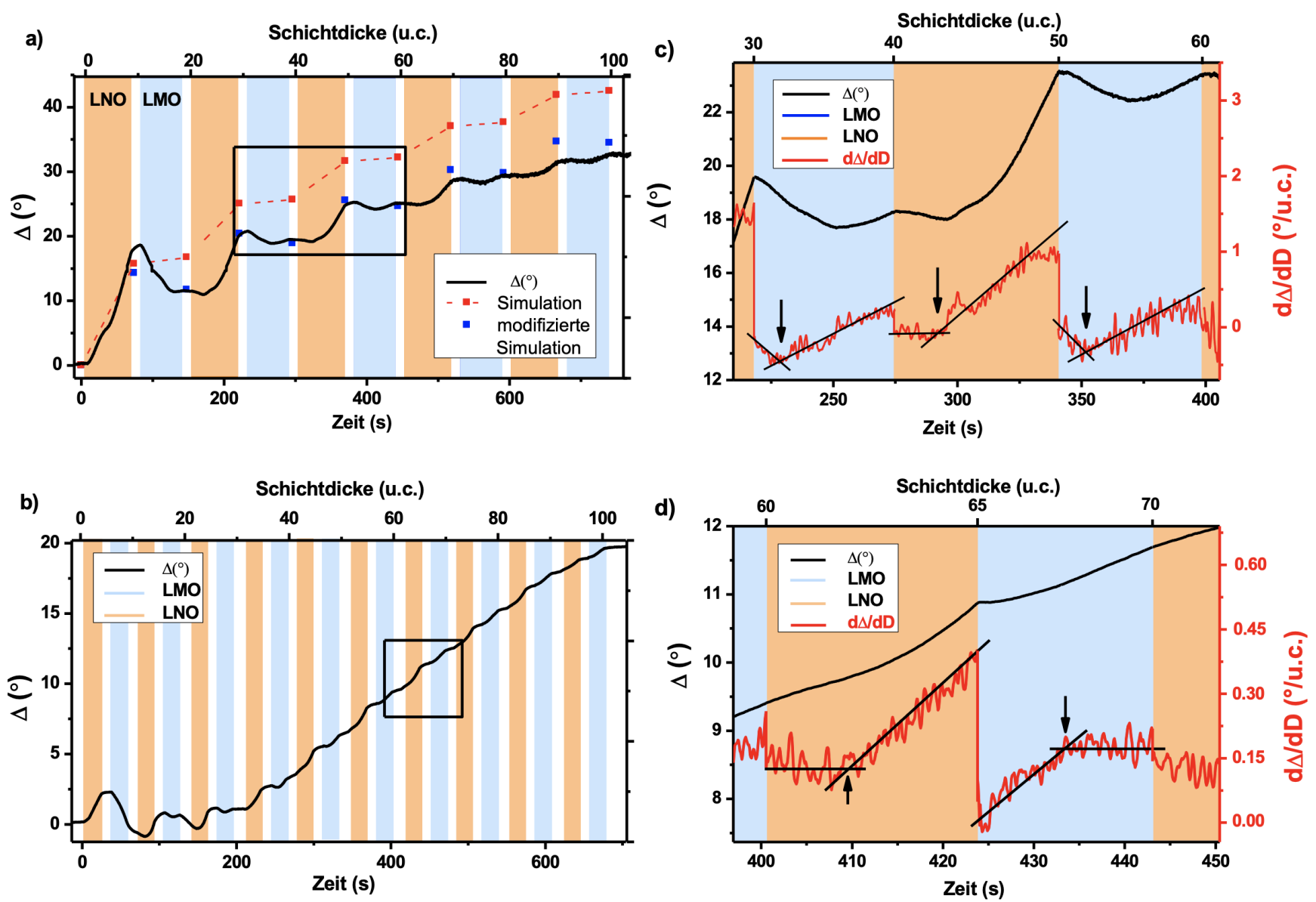

Abbildung 5.5.: Messung des Ellipsometrieparameter $\Delta$ in Abhängigkeit der Zeit und der Schichtdicke für die Übergitter a) (10/10) und b) $(5 / 5)$ mit jeweiliger Vergrößerung in c) und d). Die Orange hinterlegten Abschnitte zeigen das Signal bei der Deposition von $\mathrm{LaNiO}_{3}$, während die blauen Abschnitte die Deposition von $\mathrm{LaMnO}_{3}$ repräsentieren. Zusätzlich wird für die Messung für die (10/10) Probe der Verlauf der Simulation angefügt. In Rot das Ergebnis unter Verwendung des Brechungsindexes für die individuellen Schichten und in Blau mit einem modifizierten Brechungsindex für $\mathrm{LaMnO}_{3}$. Die vergrößerten Aufnahmen c) und d) zeigen zusätzlich die Änderung des Phasenwinkels $A=d \Delta / d D$ in Rot an. Aus diesen Abbildungen werden die Pausen zwischen den Depositionen der einzelnen Lagen zur deutlicheren Veranschaulichung entfernt. Mit Erlaubnis entnommen aus [155]. 
ander wechselwirken und so einen konstanten Brechungsindex innerhalb der Einzellage haben, keine Übereinstimmung zwischen Simulation und Messung finden. Um die Änderung des komplexen Brechungsindex des $\mathrm{LaMnO}_{3}$ und der einhergehenden elektrischen Modifizierung genauer zu untersuchen, müssen weitere Aspekte berücksichtigt werden [155].

Dafür betrachtet man die Änderung des Phasenwinkels, der in Rot in den Abbildungen 5.5 c) für (10/10) und d) für (5/5) dargestellt ist. Es zeigt sich, dass über die Deposition sämtlicher Einzelschichten charakteristische und systematische Merkmale des Signales zu erkennen sind. So verändert sich das Signal nicht homogen im Verlauf des Wachstums von $\mathrm{LaMnO}_{3}$ und $\mathrm{LaNiO}_{3}$, sondern weist innerhalb der ersten zwei bis drei Einheitszellen veränderte optische Eigenschaften auf, die nahe legen, dass die Einzelschichten als solches keine homogenen Eigenschaften besitzen [155]. In der folgenden Betrachtung sollen die Grenzflächen $\mathrm{LaMnO}_{3}$ auf $\mathrm{LaNiO}_{3}$ (NM) und andersherum (MN) gesondert betrachtet werden, beginnend mit NM.

M. Gibert et al. untersuchen vergleichbare Heterostrukturen mit der Ergebnis, dass getrieben durch die unterschiedlichen Elektronegativitäten von Mn $(1,55)$ und $\mathrm{Ni}(1,91)$ ein Ladungstransfer von Mangan auf Nickel, $\mathrm{Mn}^{3+}+\mathrm{Ni}^{3+}$ $\rightarrow \mathrm{Mn}^{4+}+\mathrm{Ni}^{2+}$, stattfindet [82,83], ähnlich wie in dem DP $\mathrm{La}_{2} \mathrm{NiMnO}_{6}$ [75-77]. Betrachtet man die geringe (teilweise sogar negative) Steigung der Phasenänderung im $\mathrm{LaMnO}_{3}$ der (10/10) Probe, gelangt man zu einem ähnlichen Ergebnis. Die veränderte Steigung von $A=d \Delta / d D$ innerhalb der ersten Einheitszellen lässt sich mit einem Elektronenverlust in der $\mathrm{LaMnO}_{3}$ Schicht erklären, der durch einen Ladungstransfer von $\mathrm{Mn}^{3+}$ auf darunter liegende $\mathrm{Ni}^{3+}$ entsteht [155]. Die große Errungenschaft der in-situ-Ellipsometrie ist es nun, dass sich durch den zeitlichen Verlauf von $\Delta(t)$, der sich zum Zeitpunkt $t^{*}$ abrupt ändert (siehe Abbildung $5.5 \mathrm{c}$ ) und d)), die charakteristische Länge des Ladungstransfers $\lambda_{C T}$ abschätzen lässt. Über:

$$
\lambda_{C T}=t^{*} / t \cdot n
$$

ergibt sich mit den gegeben Einzelschichtdicke von $n=10$ und 5 und der Depositionszeit der $\mathrm{LaMnO}_{3}$-Schicht $t_{L M O}$ für die $(10 / 10)$ Probe eine charakteristische Länge von $\lambda_{C T}^{L M O 10} \approx 2,4$ u.c. und für die (5/5) Probe 

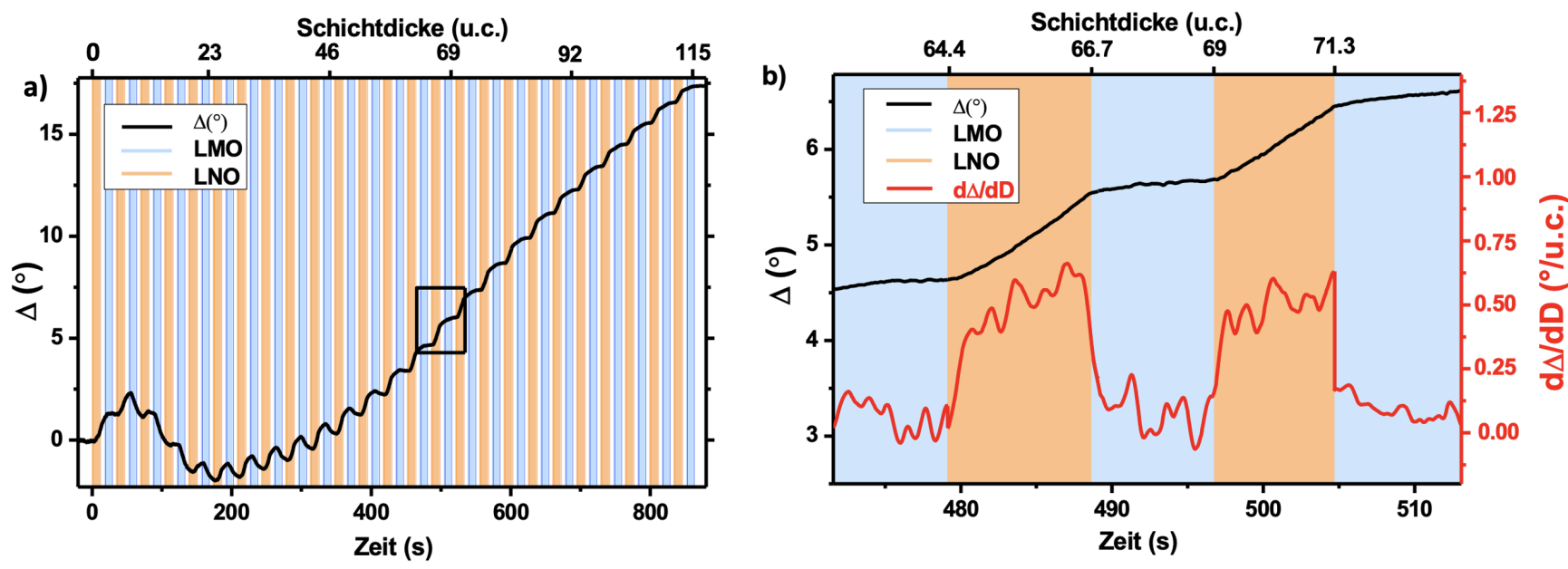

Abbildung 5.6.: Analog zu Abbildung 5.5 ist hier die Messung des Ellipsometriesignales für die $(2 / 2)$ Probe mit Vergrößerung dargestellt. Mit Erlaubnis entnommen aus [155].

$\lambda_{C T}^{L M O 5} \approx 2,7$ u.c., die der Dicke des elektronenarmen Bereiches des $\mathrm{LaMnO}_{3}$ entspricht [155]. Die ermittelten Werte stimmen somit mit den Ergebnissen aus den Untersuchungen der $\mathrm{LaMnO}_{3} / \mathrm{SrMnO}_{3}$-Übergitter überein, die mittels MAD gewachsen werden [12]. Da der Ladungstransfer auf die ersten Einheitszellen begrenzt bleibt, wächst im Anschluss $\mathrm{LaMnO}_{3}$ in seiner natürlichen $\mathrm{La}^{3+} \mathrm{Mn}^{3+} \mathrm{O}_{3}^{2-}$-Konfiguration.

Betrachtet man die entgegengesetzte Grenzfläche $\mathrm{MN}$, in der $\mathrm{LaNiO}_{3}$ auf $\mathrm{LaMnO}_{3}$ wächst, so lässt sich die geringe Steigung nicht mit dem Ladungstransfer erklären, da andernfalls die Steigung zunehmen müsste. In Verbindung mit den TEM-Bildern in Abbildung 5.3, die auf eine rauere MNOberfläche deuten, liegt hier vielmehr die Vermutung nahe, dass es sich um einen strukturellen und/oder stochiometrischen Effekt handelt, der auf eine rauere Grenzflächenbeschaftenheit zurückzuführen ist. Auch diese Annahme ist im Einklang mit bisherigen Beobachtungen von asymmetrischen Grenzflächen in $\mathrm{LaMnO}_{3} / \mathrm{LaNiO}_{3}$-Heterostrukturen, in denen die NM-Grenzfläche glatter als die MN-Grenzfläche erscheint [82,83]. Analog zur charakteristischen Länge des Ladungstransfers lässt sich die chemische Diffusionslänge $\lambda_{D}=t^{*} / t \cdot n$ bestimmen. Es ergeben sich für die (10/10) und (5/5) Probe vergleichbare Werte, $\lambda_{D}^{L N O 10} \approx 2,8$ u.c. und $\lambda_{D}^{L N O 5} \approx 2,3$ u.c.. Auch hier stellt sich hinterher das Wachstum eines unveränderten $\mathrm{LaNiO}_{3}$ ein [155]. 
Da sowohl die charakteristische Länge für den Ladungstransfer als auch die Diffusionslänge jeweils größer als zwei Einheitszellen sind, wird der Effekt der Steigerungsänderung von $\Delta(t)$ in $\mathrm{LaMnO}_{3}$ und $\mathrm{LaNiO}_{3}$ bei der $(2 / 2)$ Probe nicht beobachtet, wie in Abbildung 5.6 b) zu sehen ist. Aufgrund des Ladungstransfers des Mangan auf das darunter liegende Nickel ist das wachsende $\mathrm{LaMnO}_{3}$ elektronenarm, woraus die geringe Steigung resultiert. $\mathrm{LaNiO}_{3}$ mit $\mathrm{Ni}^{+3}$ hingegen wächst unverändert auf dem elektronenarmen $\mathrm{LaMnO}_{3}$ mit $\mathrm{Mn}^{4+}$. Der CT in das $\mathrm{LaNiO}_{3}$ kann aufgrund der Natur der Ellipsometriemessung nicht beobachtet werden, da der CT erst stattfindet, wenn das $\mathrm{LaNiO}_{3}$ bereits gewachsen ist. Somit verändern sich die optischen Eigenschaften einzelner Schichten während des Wachstums nicht und ergeben homogen gewachsene Schichten mit nahezu konstanten elektronischen Eigenschaften, die sich stark von denen der ursprünglichen $\mathrm{LaMnO}_{3}$ - und $\mathrm{LaNiO}_{3}$-Schichten unterscheiden [155]. Die durchgängige Ausbreitung der $\mathrm{Mn}^{4+} / \mathrm{Ni}^{2+}$-Kopplungen führt dazu, dass die Probe zunehmend dem Doppelperowskit $\mathrm{La}_{2} \mathrm{NiMnO}_{6}$ ähnelt. Des Weiteren können auch strukturelle Kopplungen an den Grenzflächen die Änderungen im Ellipsometriesignal erklären, die im Folgenden mit Hilfe der Raman-Spektroskopie untersucht werden sollen.

Raman-Spektroskopie Bulk $\mathrm{LaMnO}_{3}$ kristallisiert in der orthorhombischen Pbnm- und $\mathrm{LaNiO}_{3}$ der rhomboedrischen $R-3 c$-Struktur, während das B-Platz geordnete $\mathrm{La}_{2} \mathrm{NiMnO}_{6}$ in Verbindung mit der $\mathrm{Mn}^{4+} / \mathrm{Ni}^{2+}-\mathrm{Va}-$ lenzverteilung die monokline $P 12_{1} / n 1$-Struktur annimmt $[54,159,164]$. Sofern der Ladungstransfer innerhalb der ersten zwei Einheitszellen an der NM-Grenzfläche stattfindet, sollte sich dies, wie beim Doppelperowskit, auf die Struktur auswirken, da die intrinsischen rhomboedrischen und orthorhombischen Strukturen für $\mathrm{LaMnO}_{3}$ und $\mathrm{LaNiO}_{3}$ nicht mit den jeweiligen Valenzen $\left(\mathrm{Mn}^{4+} / \mathrm{Ni}^{2+}\right)$ vereinbar sind [155]. Um dies zu untersuchen, werden alle hergestellten Proben mittels polarisationsabhängiger RamanSpektroskopie untersucht. Die Ergebnisse sind in Abbildung 5.7 dargestellt. Da die Substrate ein starkes Ramansignal aufweisen, wird für jede Messung das separat gemessene STO-Spektrum abgezogen (Abbildung 5.8 a)).

Sämtliche Proben zeigen ein ähnliches Spektrum, bei dem zwei Moden stark 
ausgeprägt sind. Zum einen die starke Mode bei $674 \mathrm{~cm}^{-1}$, die aufgrund eines zusätzlichen Peaks bei $650 \mathrm{~cm}^{-1}$ leicht asymmetrisch ist. Und zum anderen eine Mode bei $527 \mathrm{~cm}^{-1}$, die eine geringere Intensität zeigt. Ein Vergleich mit dem Raman-Spektrum für B-Platz geordnetes $\mathrm{La}_{2} \mathrm{NiMnO}_{6}$ [54-56] zeigt eine gute Übereinstimmung mit den vorliegenden Spektren der Heterostrukturen. Die Moden der Einzelschichtsysteme (siehe 5.8 b)) sind nicht zu erkennen. Theoretische Rechnungen in Form von lattice dynamical calcula-

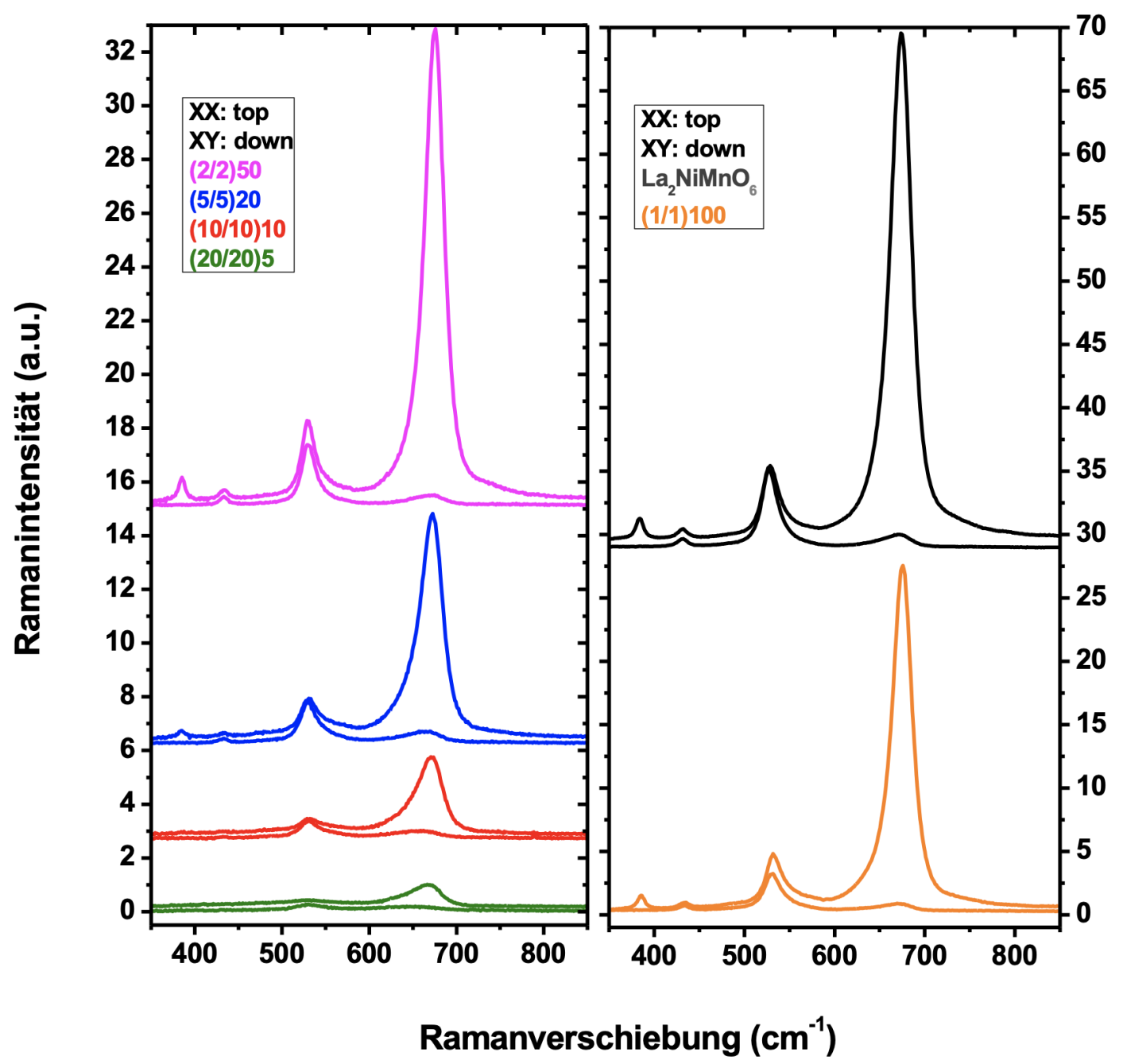

Abbildung 5.7.: Polarisationsabhängige Raman-Spektren der $\left(\mathrm{LaMnO}_{3}\right)_{n} /$ $\left(\mathrm{LaNiO}_{3}\right)_{n}$-Proben mit $\mathrm{n}=20,10,5,2$ und 1 und der SoAProbe $\mathrm{La}_{2} \mathrm{NiMnO}_{6}$. Es sei auf die unterschiedlichen Angaben der y-Skala hingewiesen, wodurch die Spektren auf der rechten Seite eine deutlich höhere Intensität aufweisen. Mit Erlaubnis entnommen aus [155]. 
a)

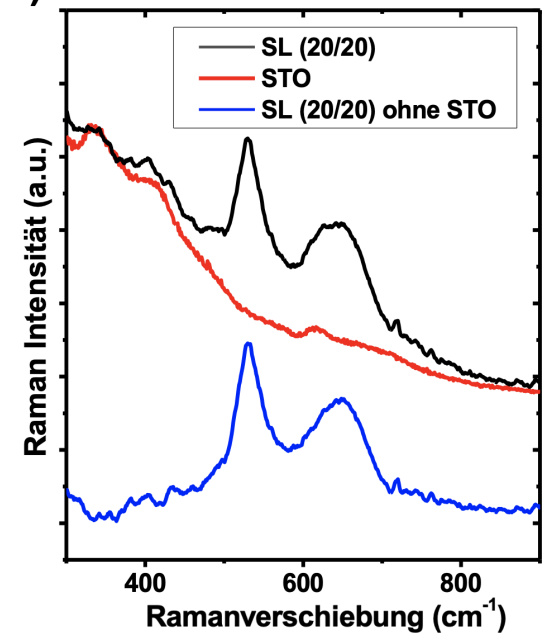

b)

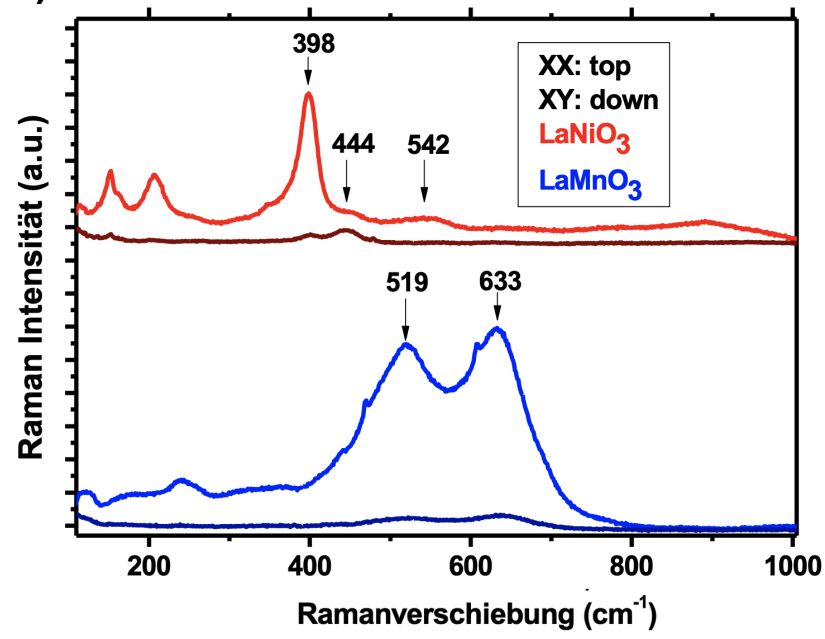

Abbildung 5.8.: a) Unpolarisiertes Raman-Spektrum der (20/20) Probe, des STO-Substrates und der alleinstehenden (20/20) Probe nach Abzug des STO-Signales. b) Polarisationsabhängige Raman-Spektren der $\mathrm{LaMnO}_{3^{-}}$und $\mathrm{LaNiO}_{3}{ }^{-}$ Einzelschichten. Mit Erlaubnis entnommen aus [155].

tions (LDC) [54-56] für das geordnete DP ergeben, dass die starke Mode bei $674 \mathrm{~cm}^{-1}$ zusammen mit der Mode bei $650 \mathrm{~cm}^{-1}$ auf die symmetrische Streckschwingung der $(\mathrm{Ni} / \mathrm{Mn}) \mathrm{O}_{6}$-Oktaeder zurückzuführen ist, die in der Literatur als Breathing Mode bezeichnet wird. Die andere Mode bei $527 \mathrm{~cm}^{-1}$ ist eine Mischmode aus antisymmetrischen Streckschwingungen und Biegebewegungen und wird als Mixed Mode bezeichnet [54-56].

Aufgrund der unterschiedlichen Struktur für das DP $\mathrm{La}_{2} \mathrm{NiMnO}_{6}$ mit BPlatzordnung (monoklin $P 12_{1} / n 1$ mit $\mathrm{Mn}^{4+} / \mathrm{Ni}^{2+}$ ) und ohne (orthorhombisch Pbnm mit $\mathrm{Mn}^{3+} / \mathrm{Ni}^{3+}$ ) weisen die Breathing und die Mixed Mode unterschiedliche Symmetrien auf. So zeigt die Breathing Mode in der B-Platz geordneten monoklinen Struktur eine $A_{g}$-Symmetrie, während in der ungeordneten orthorhombischen Struktur eine $B_{1 g}$-Symmetrie vorliegt [54-56,59]. Die Unterschiede in der Symmetrie haben Auswirkungen auf die polarisationsabhängigen Raman-Messungen. Da die $A_{g}$-Symmetrie in der XX-Konfiguration erlaubt und in der XY-Konfiguration verboten ist, ist die Breathing Mode in der XX-Konfiguration zu sehen und in der XYKonfiguration unterdrückt. Die Mixed Mode zeigt bei B-Platzordnung eine $E_{g}$-Symmetrie mit entgegengesetzten Auswahlregeln, weshalb die Mixed 
Mode bei den jeweiligen Polaristaionskonfigurationen ein entgegengesetztes Verhalten zeigt $[54-56,59]$.

Verbindet man diese Erkenntnis mit der Tatsache, dass im geordneten DP $\mathrm{La}_{2} \mathrm{NiMnO}_{6}$ die Valenz des Mangan $4+$ und die Valenz des Nickel $2+$ beträgt, lassen sich Rückschlüsse auf die Ladungsverteilung in den vorliegenden Heterostrukturen ziehen. Betrachtet man die polarisationsabhängigen Raman-Spektren in Abbildung 5.7 zeigt sich ganz klar, dass alle Proben Anzeichen der monoklinen $P 12_{1} / n 1$-Struktur aufweisen, die in Verbindung mit der $\mathrm{Mn}^{4+} / \mathrm{Ni}^{2+}$-Valenzverteilung gebracht werden muss. Darüber hinaus ist eine klare Entwicklung der Moden erkennbar. Mit abnehmender Einzelschichtdicke $n$ nimmt die Intensität der Mixed und Breathing Mode in der jeweiligen Polarisationskonfiguration systematisch zu, was durch das Intensitätsverhältnis zur (1/1) Probe, $I_{n} / I_{1}$, in Tabelle $5.2 \mathrm{zu}$ erkennen ist. Zusätzlich reduziert sich deren Halbwertsbreite (FWHM, engl.; full width on half maximum), was eine erhöhte kristalline Qualität der Phase nahelegt. Der Verdacht des Ladungstransfers an der NM-Grenzfläche wird durch die Raman-Messungen weiter bekräftigt. Es zeigt sich, dass der Ladungstransfer mit einer strukturellen Kopplung einhergehen muss, in dessen Folge sich bei Reduzierung der Einzelschichtdicke $n$ die Doppelperowskiphase des $\mathrm{La}_{2} \mathrm{NiMnO}_{6}$ stetig über die gesamte Probe ausbreitet [155].

Tabelle 5.2.: Ergebnisse der polarisationsabhängigen Raman-Spektroskopie: FWHM und die Position der Breathing und Mixed Mode in der XX-Konfiguration. Messungen mit * beinhalten den Peak bei $650 \mathrm{~cm}^{-1}$. Mit Erlaubnis nach [155].

\begin{tabular}{|c|c|c|c|c|}
\hline & $\begin{array}{r}\text { FWHM } \\
\text { Brea }\end{array}$ & $\begin{array}{l}\text { Intensität } \\
\text { Mode }\end{array}$ & $\begin{array}{r}\text { FWHM } \\
\mathrm{Mi}\end{array}$ & $\begin{array}{l}\text { ntensität } \\
\text { ode }\end{array}$ \\
\hline & {$\left[\mathrm{cm}^{-1}\right]$} & $I_{n} / I_{1}$ & {$\left[\mathrm{~cm}^{-1}\right]$} & $I_{n} / I_{1}$ \\
\hline $20 / 20$ & $29 / 48^{*}$ & 0,07 & 58 & 0,03 \\
\hline $10 / 10$ & $26 / 37^{*}$ & 0,1 & 31 & 0,12 \\
\hline $5 / 5$ & $24 / 31^{*}$ & 0,26 & 28 & 0,3 \\
\hline $2 / 2$ & 26 & 0,48 & 20 & 0,65 \\
\hline $1 / 1$ & 26 & 1 & 25 & 1 \\
\hline SoA & 27 & - & 22 & - \\
\hline
\end{tabular}


Magnetische Messungen Schließlich sollen auch die magnetischen und elektrischen Eigenschaften der Heterostrukturen betrachtet werden. Die feldund temperaturabhängigen Magnetisierungsmessungen $(M(H)$ bei $T=5 \mathrm{~K}$ und $M(T) / M(5 K)$ bei $H=100 O e)$ sind in Abbildung 5.9 dargestellt. Die Werte der feldabhängigen Magnetisierung werden dabei auf eine Einheitszelle normiert, die sich im Mittel aus einer halben Einheitszelle mit Mangan und mit Nickel zusammensetzt, 1 u.c. $=0,5$ u.c. $\mathrm{Mn}+0,5$ u.c. $\mathrm{Ni}$.

In Abbildung 5.9 a) sind zusammengefasst die Messungen für die Einzelschicht aus $\mathrm{LaMnO}_{3}$ und die (20/20) Probe aufgetragen. Die (20/20) Probe zeigt eine Übergangstemperatur von $T_{C}=161 K$ (logarithmische Ableitung in Abbildung A.4 im Anhang zu sehen) und eine Sättigung von $M_{\text {sat }}=$ $1,65 \mu_{B} /$ u.c. $^{3}$. Dieses Verhalten entspricht weitestgehend den magnetischen Eigenschaften des $\mathrm{LaMnO}_{3}$ mit $T_{C}=158 \mathrm{~K}$ und $M_{\text {sat }}=2,8 \mu_{B} / M n$. Somit wird das magnetische Verhalten der (20/20) Probe durch die Eigenschaften des $\mathrm{LaMnO}_{3}$ dominiert und zeigt, dass die involvierten Materialsysteme $\mathrm{LaMnO}_{3}$ und $\mathrm{LaNiO}_{3}$ ihre intrinsischen Eigenschaften beibehalten und das Verhalten der Heterostruktur bestimmen. Dies spiegelt sich auch in der elektrischen Leitfähigkeit wider (siehe Abbildung A.5 im Anhang), in dem die Heterostruktur mit $n=20$ metallisches Verhalten aufweist, was auf das elektrisch leitende $\mathrm{LaNiO}_{3}$ zurückzuführen ist ${ }^{4}$. Eine Kopplung zwischen $\mathrm{LaMnO}_{3}$ und $\mathrm{LaNiO}_{3}$ ist nicht zu erkennen.

Dies ändert sich jedoch für die Heterostruktur mit $n=10$, dessen Einzelschichtdicke auf $d_{n=10}=2,2 \mathrm{~nm}$ reduziert wird. Betrachtet man die temperaturabhängige Messung in Abbildung 5.9 b) sind zwei Übergangstemperaturen zu erkennen, die auf zwei unabhängige ferromagnetische Phasen deuten. Die erste Phase mit $T_{C, \text { low }}=155 K$ hat, wie auch schon in der $(20 / 20)$ Probe, ihren Ursprung in der $\mathrm{LaMnO}_{3}$-Einzelschicht. Die zweite Phase hat eine höhere Übergangstemperatur $T_{C, h i g h}=260 \mathrm{~K}$ und trägt insgesamt weniger zum gesamten magnetischen Moment der Probe bei. Betrachtet man die magnetischen Eigenschaften des Doppelperowskit $\mathrm{La}_{2} \mathrm{MnNiO}_{6}$ mit $T_{C, L N M O}=280 \mathrm{~K}$ und trägt dies mit den Erkenntnissen über den La-

\footnotetext{
${ }^{3}$ was einer Magnetisierung von $M_{\text {sat }}=3,3 \mu_{B} / M n$ und $0 \mu_{B} / N i$ entspricht.

${ }^{4}$ bei $n=20$ beträgt die Einzelschicht innerhalb der Heterostruktur eine Dicke von $d_{n=20}=4,4 \mathrm{~nm}$.
} 
dungstransfer an der NM-Grenzfläche der vorhergehenden Untersuchungen zusammen, kommt man zu der Schlussfolgerung, dass die Hochtemperaturphase durch das Auftreten des Superaustausches zwischen $\mathrm{Mn}^{4+}-\mathrm{O}-\mathrm{Ni}^{2+}$

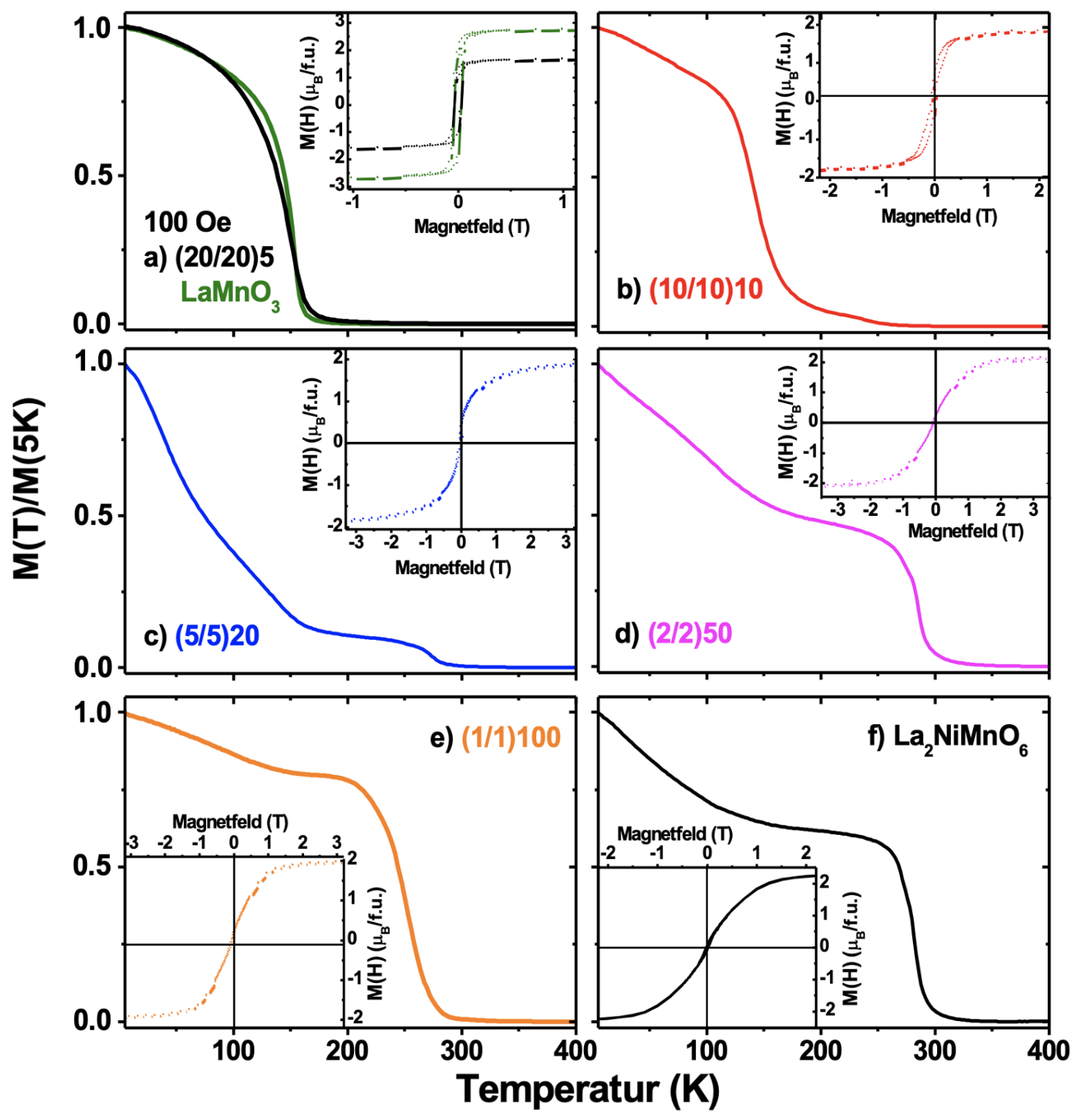

Abbildung 5.9.: Temperatur- und Feldabhängige Messungen der a) $\mathrm{LaMnO}_{3}$-Einzelprobe mit $d_{L M O}=16 \mathrm{~nm}$ in Grün und $(20 / 20)$ Probe in Schwarz, b) (10/10) Blau, c) (5/5) Magenta, d) (2/2) Lila, e) (1/1) Orange und f) SoA-Probe Schwarz. Die $M(T)$-Messungen werden bei einem angelegtem Feld von $H=100 O e$ und die $M(H)$ Messungen bei einer Temperatur von $T=5 K$ angefertigt. Mit Erlaubnis entnommen aus [155]. 
an der Grenzfläche entstehen muss. Die Einzelschichtsysteme koppeln somit miteinander, was sich magnetisch über das Auftreten der $\mathrm{La}_{2} \mathrm{NiMnO}_{6}$-Phase bemerkbar macht. Diese Kopplung beeinflusst auch die elektrischen Eigenschaften, sodass ein Übergang vom metallischen Verhalten für $n=20$ in den isolierenden Zustand für $\mathrm{n}=10$ beobachtet wird, siehe Abbildung A.5.

Entsprechend dominieren die Eigenschaften der $\mathrm{La}_{2} \mathrm{NiMnO}_{6}-\mathrm{Phase}$ bei einer weiteren Reduzierung der Einzelschichtdicke auf $n=5\left(d_{n=5}=1,1 \mathrm{~nm}\right)$ weiter die Eingenschaften der Probe und unterdrücken die intrinsische $\mathrm{LaMnO}_{3}$ Phase, was sich in den Übergangstemperaturen niederschlägt. Die Doppelperowskitphase zeigt eine erhöhtes $T_{C}$ mit $278 \mathrm{~K}$ und eine abgesenkte Übergangstemperatur der $\mathrm{LaMnO}_{3}$-Schicht, $T_{C, l o w}=146 \mathrm{~K}$. Die $(2 / 2)$ Probe wird schließlich durch die Grenzflächenphase dominiert und zeigt mit $T_{C, h i g h}=285 \mathrm{~K}$ die nahezu identische Übergangstemperatur wie die SoA-Probe $T_{C}=287 K$ (Abbildung 5.9 f) ) [155]. Jedoch erreicht die Sättigungsmagnetisierung mit $M_{\text {sat }}=2,1 \mu_{B} /$ u.c. nicht den zu erwartenden Wert für vollständig geordnetes $\mathrm{La}_{2} \mathrm{NiMnO}_{6}$ mit $M_{\text {sat }}=2,5 \mu_{B} /$ u.c. $(=$ $\left.5 \mu_{B} /(M n+N i)\right)$, was auf die Anwesenheit antiferromagnetischer $\mathrm{Ni}^{2+}$ $\mathrm{O}-\mathrm{Ni}^{2+}$ und $\mathrm{Mn}^{4+}-\mathrm{O}-\mathrm{Mn}^{4+}$ Kopplungen hinweist. Schließlich lässt sich aus dem temperaturabhängigen Verhalten der (1/1) Probe ebenfalls die DPPhase erkennen. Jedoch wird auch hier nicht die maximale Sättigung erreicht, was auf eine unvollständige $\mathrm{Ni} / \mathrm{Mn}$-Ordnung hindeutet. Das soll im nächsten Unterkapitel 5.1.2 gesondert betrachtet und diskutiert werden.

Zusammenfassung und Diskussion Zur vereinfachten Veranschaulichung der Ergebnisse werden die magnetischen Eigenschaften (Sättigungsmagnetisierung und Übergangstemperatur) und die Ramanintensität der Breathing Mode der Heterostrukturen und der SoA-Probe (mit $1 / n=1$ ) gegen die Grenzflächendichte $\Lambda=1 / n$ in Abbildung 5.10 aufgetragen. Diese Darstellung zeigt den kontinuierlichen strukturellen und magnetischen Übergang aus den Einzelschichtsystemen hin zum Doppelperowskit $\mathrm{La}_{2} \mathrm{NiMnO}_{6}$. Über die in-situ-Ellipsometrie lässt sich die Bildung der DP-Phase an der NMGrenzfläche $\left(\mathrm{LaMnO}_{3}\right.$ auf $\left.\mathrm{LaNiO}_{3}\right)$ über den Ladungstransfer beobachten und dessen Ausbreitung über die charakteristische Länge $\lambda_{C T} \approx 2-3$ u.c. abschätzen. Die Ausdehnung von zwei bis drei Einheitszellen hat zur Folge, 


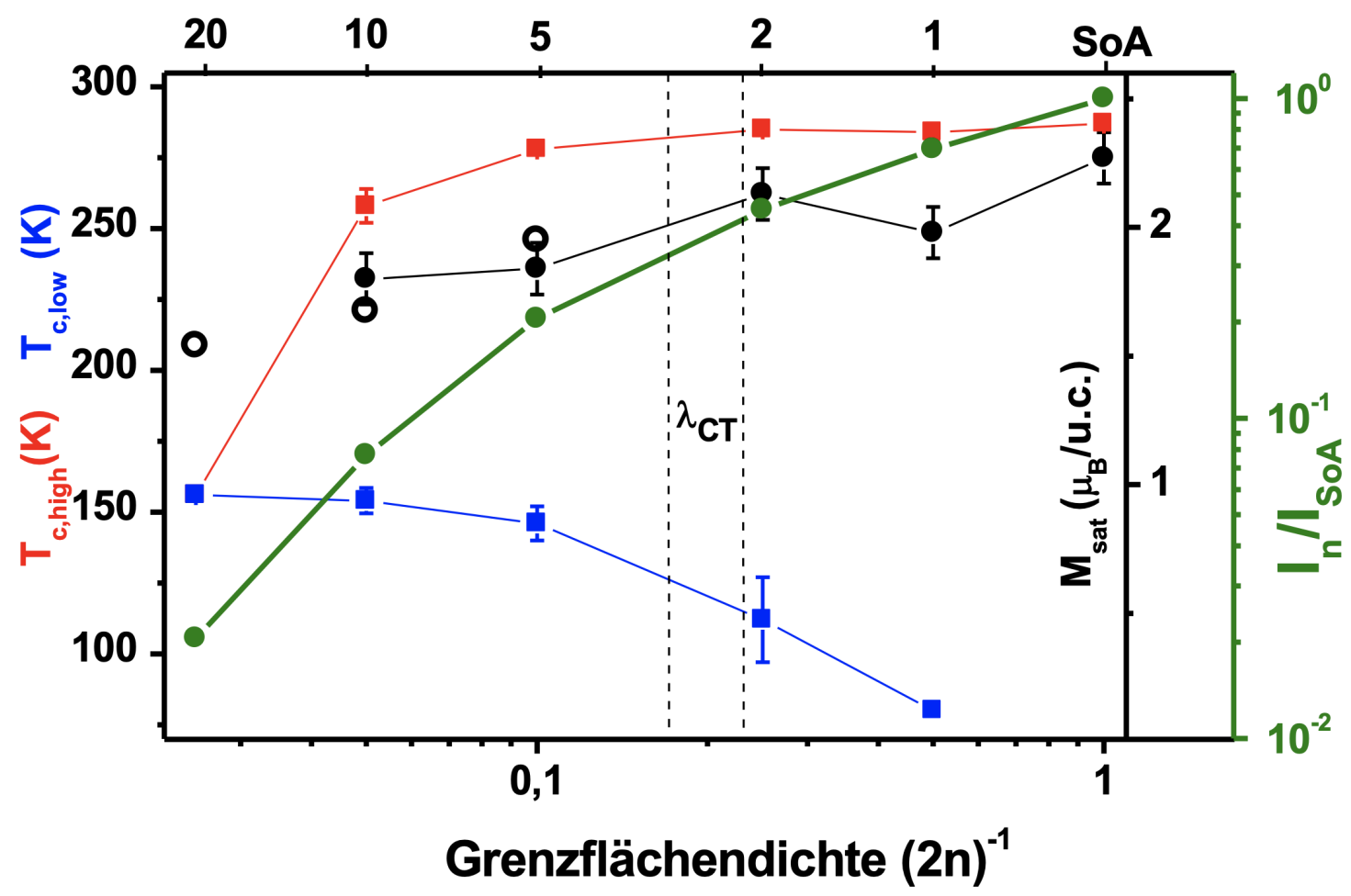

Abbildung 5.10.: $M_{\text {sat }}$ (Schwarz), $T_{C, \text { low }}$ (Blau), $T_{C \text {,high }}$ (Rot) und $I_{n} / I_{\text {SoA }}$ (Grün) der Heterostrukturen gegen die Grenzflächendichte $\Lambda=1 / n$. Zusätzlich sind theoretisch errechneten Sättigungsmagnetisierungen mit schwarzen Kreisen eingetragen. Mit Erlaubnis entnommen aus [155].

dass sich für die Heterostrukturen mit einer Einzelschichtdicke von $n>\lambda_{C T}$ zwei magnetische Phasen beobachten lassen, die 1. auf die Grenzflächeneffekte und 2. auf die $\mathrm{LaMnO}_{3}$-Lagen zurückzuführen sind. Heterostrukturen mit $n<\lambda_{C T}$ hingegen verhalten sich aufgrund ihrer dominierenden Grenzflächeneffekte, die sich durch die gesamte Probe durchziehen, wie geordnetes DP $\mathrm{La}_{2} \mathrm{NiMnO}_{6}$ mit ferromagnetischem Superaustausch über die $\mathrm{Mn}^{4+}-\mathrm{O}-$ $\mathrm{Ni}^{2+}$ Bindungen.

Darüber hinaus zeigt sich über den Ellipsometrieverlauf und die TEMMessungen, dass die beiden Grenzflächen $\left(\mathrm{LaMnO}_{3}\right.$ auf $\mathrm{LaNiO}_{3}(\mathrm{NM})$ und andersherum (MN)) nicht äquivalent sind, was die vorhergehenden Untersuchungen in der Literatur $[82,83]$ bestätigen. Die Steigungsänderungen in der Ellipsometrie-Messung offenbaren, dass die glatte NM-Grenzfläche den Ladungstransfer ermöglicht, während er bei der vergleichsweise rauen Grenzflä- 
che unterdrückt zu sein scheint. Dies eröffnet die Vermutung, dass auch die strukturelle Kopplung, die bei der Raman-Spektroskopie beobachtet wird, nur an der NM-Grenzfläche in Verbindung mit dem Ladungstransfer stattfindet. Der Einfluss der unterschiedlichen Kopplungen an den jeweiligen Grenzflächen auf die Proben mit $n=10,5$ und 2 ist schematisch in Abbildung 5.11 dargestellt. Für die $(2 / 2)$ Probe mit $n<\lambda_{C T}$ ergibt sich eine homogene Probe mit $\mathrm{Ni}^{2+}$ und $\mathrm{Mn}^{4+}$ in der monoklinen $P 12_{1} / n 1$-Struktur. Diese Phase baut sich in den Proben (5/5) und (10/10) mit $n>\lambda_{C T}$ nur an der NM-Grenzfläche auf, während die restliche Einzellagen ihre fast intrinsischen $\mathrm{LaMnO}_{3}$ - und $\mathrm{LaNiO}_{3}$-Eigenschaften behalten und an der $\mathrm{MN}$ Grenzfläche nicht miteinander koppeln.

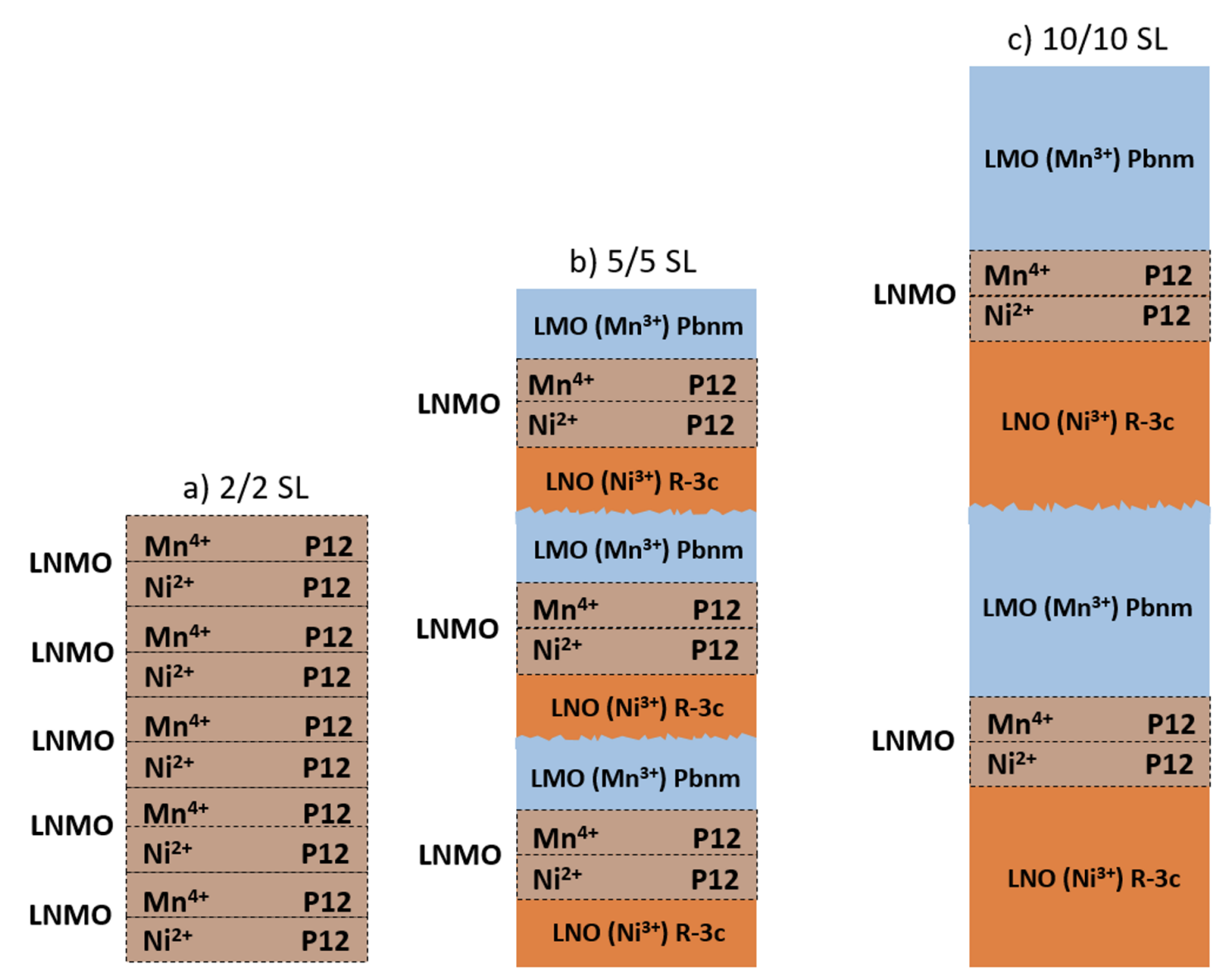

Abbildung 5.11.: Schematische Darstellung der Grenzflächeneffekte in den $\left(\mathrm{LaMnO}_{3}\right)_{n} /\left(\mathrm{LaNiO}_{3}\right)_{n}$-Heterostrukturen mit $n=10,5$ und 2. Mit Erlaubnis entnommen aus [155]. 
Unter Verwendung des in Abbildung 5.11 dargestellten Modelles, lassen sich die theoretischen Sättigungsmagnetisierungen vorhersagen. Dabei wird davon ausgegangen, dass das intrinsische $\mathrm{LaNiO}_{3}$ keinen magnetischen Beitrag liefert, während $\mathrm{LaMnO}_{3}$ eine Magnetisierung von $M_{\text {sat }, L M O}=2,8 \mu_{B} /$ u.c. $=$ 2,8 $\mu_{B} / M n$ mitbringt und die NM-Grenzfläche die Magnetisierung des DP $\mathrm{La}_{2} \mathrm{NiMnO}_{6} M_{\text {sat }, L N M O}=2,5 \mu_{B} /$ u.c. $=5 \mu_{B} / M n+N i$ aufweist. Über das Verhältnis der jeweiligen Schichtdicken $\left(\mathrm{LaNiO}_{3}: \mathrm{La}_{2} \mathrm{NiMnO}_{6}: \mathrm{LaMnO}_{3}\right)$ $=(1 / 4: 2 / 4: 1 / 4)$ für $n=5,(3 / 8: 2 / 8: 3 / 8)$ für $n=10$ und schließlich $(7 / 16: 2 / 16: 7 / 16)$ für $n=20$, ergeben sich für die Proben die Werte [155]:

$$
\begin{aligned}
M_{\text {sat }}(20 / 20) & =1,54 \mu_{B} / \text { u.c. } \\
M_{\text {sat }}(10 / 10) & =1,675 \mu_{B} / \text { u.c. } \\
M_{\text {sat }}(5 / 5) & =1,95 \mu_{B} / \text { u.c. }
\end{aligned}
$$

Die ermittelten Werte sind zusätzlich in Abbildung 5.10 dargestellt. Aus der Abbildung wird deutlich, dass diese Werte gut zu den experimentell beobachteten Ergebnissen passen. Würden beide Grenzflächen zum magnetischen Moment beitragen, würden deutliche Abweichungen entstehen, da beispielsweise bereits für die (5/5) Probe die volle Magnetisierung des Doppelperowskites von $M_{\text {sat }}(5 / 5)=2,5 \mu_{B} /$ u.c. beobachtet werden sollte.

Hervorzuheben ist zu allerletzt, dass die vorliegenden Heterostrukturen, die mit der MAD gewachsen werden, erstmals die Doppelperowskitphase an der Grenzfläche zeigen. Die vorhergehende Untersuchungen an dem selben Materialsystem, die allerdings über Magnetron-Sputtering hergestellt werden, haben ebenfalls den Ladungstransfer beobachten und nachweisen können. Jedoch bleibt die Bildung der DP-Phase aus. Eine mögliche Ursache liegt in den besonderen Wachstumsbedingungen innerhalb der MAD, die sich im Vergleich zum Wachstum beim Sputtering nah dem Gleichgewichtszustand befinden, wie in Kapitel 3 erläutert. Das Wachstum aus der Flüssigphase bei hohen Temperaturen muss im Vergleich zu den konventionellen Wachstumsmethoden die strukturelle Kopplung und damit verbunden die Bildung des Superaustausches zwischen $\mathrm{Mn}^{4+}-\mathrm{O}-\mathrm{Ni}^{2+}$ ermöglichen, die bei den vorhergehenden Untersuchungen $[82,83]$ trotz $\mathrm{CT}$ nicht beobachtet wird. 


\subsubsection{Kationordnung in $\mathrm{LaNiO}_{3} / \mathrm{LaMnO}_{3}-$ Heterostrukturen mittels systematischer Reduzierung der Einzelschichtdicke}

Nachdem ausführlich über die Grenzflächeneffekte in den $\mathrm{LaMnO}_{3} / \mathrm{LaNiO}_{3}$ Heterostrukturen eingegangen wurde, soll nun gesondert die B-Platzordnung in der $(1 / 1)$ Probe, bzw im Folgenden als LbL (engl.; layer by layer) bezeichnete Probe, betrachtet werden, die über die systematische Reduzierung der Einzelschichtdicke $n$ erreicht wird.

Strukturelle und magnetische Eigenschaften Abbildung 5.12 zeigt die XRD-Messung der LbL (( $\left.\left.\mathrm{LaMnO}_{3} / \mathrm{LaNiO}_{3}\right)_{100}\right)$ und der SoA-Referenzprobe $\mathrm{La}_{2} \mathrm{NiMnO}_{6}$ mit einer Schichtdicke von $d_{S o A}=79,7 \mathrm{~nm}$. Mit einer Schichtdicke von $d_{L b L}=44,1 \mathrm{~nm}$ für die LbL-Probe wird die erwartete Gesamtschichtdicke mit einer geringen Abweichung von $\Delta d=-0,05 \%$ erreicht. Auch die out-of-plane Gitterkonstante $c_{L b L}=0,3808 \mathrm{~nm} \approx 0,3810 \mathrm{~nm}=$ $c_{S o A}$ entspricht weitestgehend der Gitterkonstanten der SoA-Probe (siehe beides in Tabelle 5.1) und gibt damit keine Anhaltspunkte dafür, dass das stöchiometrische Verhältnis zwischen $\mathrm{LaMnO}_{3}$ und $\mathrm{LaNiO}_{3}$ nicht stimmt ${ }^{5}$. Der ( 111 1)-Strukturpeak der SoA-Probe zeigt im Gegensatz zur LbL-Probe eine leichte $K_{\alpha, 1} / K_{\alpha, 2}$-Aufspaltung, was auf die hohe kristalline Qualität der SoA-Probe deutet, aber auch auf die vergleichsweise große Schichtdicke zurückzuführen ist. Zudem weist die SoA-Probe einen (1/2 1/2 1/2)Überstrukturpeak auf, der bei B-Platz geordneten Doppelperowskiten in der Verdopplung der Einheitszelle in (111)-Richtung begründet ist. Für die SoA-Probe lässt sich somit über die XRD-Messung ein erster Ordnungsgrad der SoA-Probe mit $S=I\left(\begin{array}{llll}1 / 2 & 1 / 2 & 1 / 2\end{array}\right) / I\left(\begin{array}{lll}1 & 1 & 1\end{array}\right)=5,53 \cdot 10^{-4}$ abschätzen. Wie bereits in Kapitel 2.2 erläutert, muss der Ordnungsgrad, der über das Intensitätsverhältnis bestimmt wird, mit Vorsicht betrachtet werden und kann durch weitere Faktoren beeinflusst werden, wie später diskutiert wird. Aus diesem Grund darf aus dem fehlenden Überstrukturpeak der LbL-Probe kein voreiliger Rückschluss auf die B-Platzordnung gezogen werden. Zumal die temperaturabhängige Messung der Magnetisie-

\footnotetext{
${ }^{5}$ Auf diesen Zusammenhang wird im nächsten Kapitel 5.2 genauer eingegangen.
} 


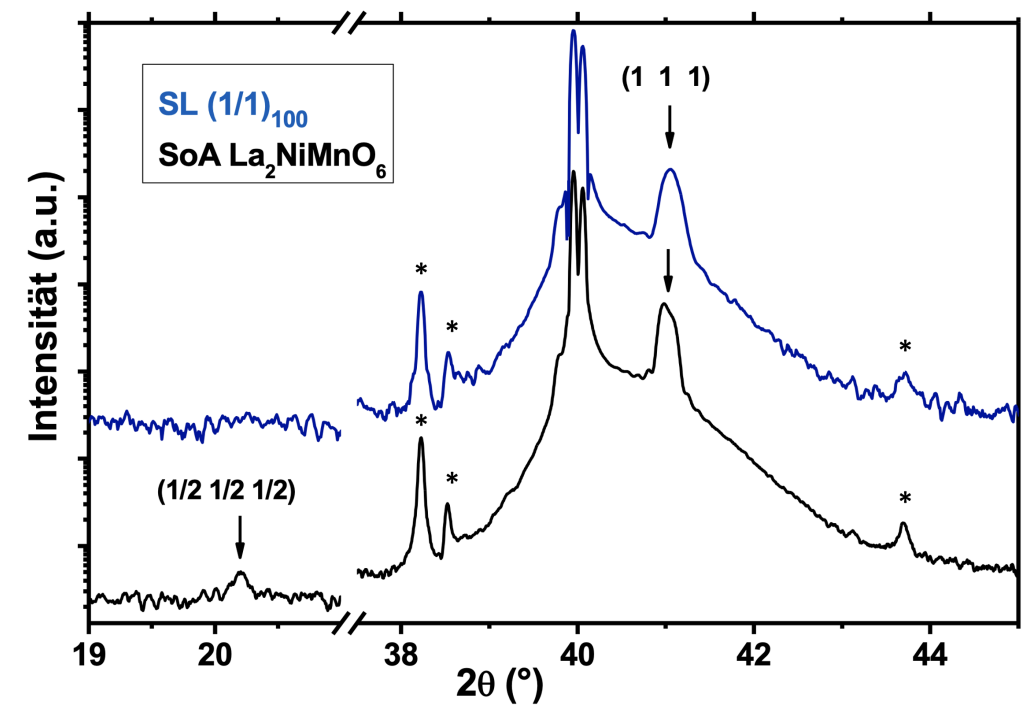

Abbildung 5.12.: XRD-Messungen der LbL- und SoA-Probe, wie sie auch schon in Abbildung A.3 dargestellt sind. Die mit * markierten Reflexe sind Messartefakte der Röntgenanlage, die auf nicht vollständig monochromatische Strahlung und den Probenhalter aus Aluminium zurückzuführen sind. Mit Erlaubnis entnommen und bearbeitet aus [155].

rung (Abbildung 5.13) keine Anzeichen auf eine ausgeprägte ungeordnete $\mathrm{LaMn}_{0,5} \mathrm{Ni}_{0,5} \mathrm{O}_{3}$-Phase mit erniedrigter Übergangstemperatur zeigt. Vielmehr erreicht die LbL-Probe eine ähnlich hohe Übergangstemperatur wie die SoA-Probe, $T_{C, L b L}=284 K$ und $T_{C, S o A}=287 K$. Jedoch weisen beide Proben, sowohl die LbL $\left(M_{s a t, L b L}=1,9 \mu_{B} /\right.$ u.c. $)$ als auch die SoA $\left(M_{\text {sat }, L b L}=2,23 \mu_{B} /\right.$ u.c. $)$, nicht den theoretischen Maximalwert für das magnetische Moment mit $M_{\text {sat }}=2,5 \mu_{B} /$ u.c. auf.

Diskussion der synthetischen Kationordnung Die SoA-Referenzprobe zeigt einen (1/2 1/2 1/2)-Überstrukturpeak, der eine erste Einschätzung der B-Platzordnung mittels $S=I\left(\begin{array}{lll}1 / 2 & 1 / 2 & 1 / 2\end{array}\right) / I\left(\begin{array}{lll}1 & 1 & 1\end{array}\right)=5,53 \cdot 10^{-4}$ zulässt. Dieser Wert liegt jedoch unterhalb des theoretischen Wertes $S_{\text {Theo }}=10^{-2}$ und auch unterhalb bisher experimentell beobachteter Werte für Proben der MAD $\left(S_{L N M O}=2,2 \cdot 10^{-3}\right)[62]$. Die Intensität des Überstrukturreflexes ermöglicht jedoch keine zuverlässige Einschätzung der B-Platzordnung, da weitere Faktoren Einfluss auf den Peak haben können und diesen sowohl verstärken als auch abschwächen können. Wie bereits mehrfach erwähnt, 
kann die Präsenz von Antiphasengrenzen den Reflex deutlich abschwächen. Andererseits kann sich auch der entgegengesetzte Trend durchsetzen und ein Peak vorhanden sein (bzw. der Peak verstärkt werden), obwohl keine B-Platzordnung vorliegt. Ermöglicht werden kann dies durch strukturelle Verzerrungen, Verkippungen der $\mathrm{B} / \mathrm{B}^{\prime} \mathrm{O}_{6}$-Oktaeder sowie Verschiebungen der A-Kationen, B-Kationen und $\mathrm{O}^{2-}$-Anionen innerhalb der Überstruktur der NaCl-Anordnung in der (111)-Richtung. Bei einer entsprechenden Abstandsmodulation kann es so zu einer Nachahmung der Überstruktur in den Doppelperowskiten kommen $[6,62,67,72,74]$, die sich in dem Überstrukturpeak widerspiegelt.

Zur zuverlässigeren Einschätzung der B-Platzordnung wird daher die Sättigungsmagnetisierung der SoA-Probe verwendet. Mit $M_{\text {sat }, \text { SoA }}=2,23 \mu_{B} /$ u.c. liegt auch diese unterhalb des theoretischen Maximalwertes, sodass ein Ordnungsgrad für die Ni- und Mn-Kationen von 89,2 \% erreicht wird. Diese unvollständige B-Platzordnung lässt sich auf ein nicht optimales stöchiometrisches Verhältnis der beteiligten Präkusoren in der Lösung zur Herstellung der SoA-Schicht zurückführen. Vergangene Untersuchungen an Proben der MAD haben jedoch bereits gezeigt, dass sich die Wachstumsbedingungen weiter optimieren lassen und ein höherer Ordnungsgrad möglich ist [62].
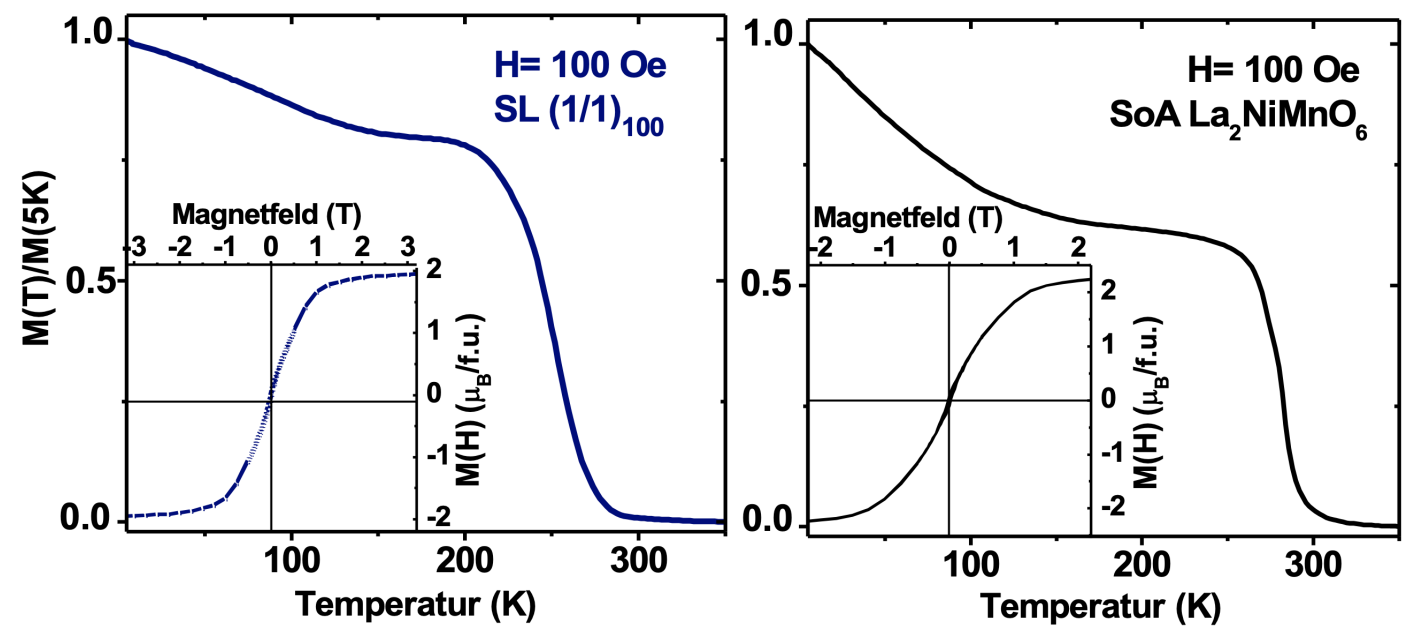

Abbildung 5.13.: Magnetisierungsmessungen der LbL- und SoA-Probe, wie sie auch schon in Abbildung 5.9 dargestellt sind. Mit Erlaubnis entnommen und bearbeitet aus [155]. 
Entscheidend bleibt die Untersuchung des erreichten Ordnungsgrades der LbL-Probe durch die systematische Reduzierung der Einzelschichtdicken. Aus erwähnten Gründen wird das Ausbleiben des Überstrukturpeaks zur Einschätzung des Ordnungsgrades nicht weiter berücksichtigt. Dennoch, trotz der weitestgehend genau erzielten Schichtdicke für die LbL-Probe und der hohen Übergangstemperatur $\left(T_{C, L b L}=284 \mathrm{~K}\right)$ deutet die geringe Sättigungsmagnetisierung $\left(M_{s a t, L b L}=1,9 \mu_{B} /\right.$ u.c. $)$ auf eine unvollständige B-Platzordnung hin, die sich auf $76 \%$ abschätzen lässt. Selbst durch die scheinbar präzise Wachstumskontrolle der Heterostrukturen gelang es bisher wahrscheinlich nicht, eine vollständig geordnete LbL-Probe zu erhalten. Die Ursache dafür kann im System selbst und der damit verbundenen Wachstumskinetik begründet sein. Wie die Untersuchungen an den $\left(\mathrm{LaMnO}_{3}\right)_{n} /$ $\left(\mathrm{LaNiO}_{3}\right)_{n}$-Heterostrukturen mit $n>2$ und vorhergehende Untersuchungen anderer Gruppen gezeigt haben [82,83], wächst $\mathrm{LaNiO}_{3}$ vergleichsweise rau auf dem $\mathrm{LaMnO}_{3}$ (MN-Grenzfläche). Dies kann große Auswirkungen auf das Wachstum von Lagen mit der Dicke von nur einer Einheitszelle haben, indem die Ausbildung eines geschlossenen Filmes mit der Schichtdicke von nur einer Einheitszelle verhindert wird. Somit wird die vollständige BPlatzordnung unterdrückt. Um zu untersuchen, ob sich die B-Platzordnung über die lagenweise Deposition der Einzelschichtsysteme in anderen Systemen künstlich erzeugen lässt, werden im nächsten Kapitel die Erfahrungen auf weitere Systeme angewandt. 


\subsection{Kationordnung in 3d-Doppelperowskiten und Heterostrukturen durch stöchiometrischen Lagenwachstum}

Rechtliches und Lizenzen Das folgende Kapitel fasst einen weiten Teil der wissenschaftlichen Ergebnisse der vorliegenden Promotion zusammen. Große Teile davon wurden bereits in der Publikation „B-site Cation Ordering in Films, Superlattices and Layer-by-Layer Grown Double Perovskites" [63] veröffentlicht, in dem ich als Erstautor angegeben bin und den Hauptanteil der Ergebnisse produziert habe. Die Ergebnisse wurde unter der Creative Common CC BY Lizenz veröffentlicht, womit es mir als Autor freisteht die Abbildungen, Tabellen und inhaltliche Formulierungen zu verwenden. Endsprechende Stellen werden zitiert. Messungen und Abbildungen, die nicht direkt von mir stammen, werden entsprechend gekennzeichnet.

\subsection{1. $\mathrm{La}_{2} \mathrm{CoMnO}_{6}$ und $\mathrm{LaCoO}_{3} / \mathrm{LaMnO}_{3}-$ Heterostrukturen}

Im folgenden Kapitel sollen die Ergebnisse der künstlich hergestellten BPlatzordnung in $\mathrm{La}_{2} \mathrm{CoMnO}_{6}$ vorgestellt werden, die im Gegensatz zu dem vorherigen Kapitel durch systematische Änderung der Lagenstöchiometrie erzielt wird. Die gewonnen Erfahrungen werden anschließend auf das Doppelperowskit $\mathrm{Sr}_{2} \mathrm{TiRuO}_{6}$ übertragen und erprobt.

Einzelschichtsystem $\mathrm{LaCoO}_{3}$ Die Wachstumsbedingungen für $\mathrm{LaMnO}_{3}$ wurden bereits im vorherigen Kapitel vorgestellt und werden unverändert für die vorliegenden Proben angewandt. Die folgenden Proben werden jedoch mit leicht erhöhter Depositionstemperatur von $950^{\circ} \mathrm{C}$ hergestellt, was die Eigenschaften des $\mathrm{LaMnO}_{3}$ nicht verändert.

In $\mathrm{LaCoO}_{3}$ führt ein empfindliches Zusammenspiel aus der Kristallfeldaufspaltung und der Hund'schen Kopplung zu einem nicht trivialen Spinzustand, der in Abhängigkeit von der Temperatur sämtliche Spinzustände (low-, intermediate-, high-Spinzustand und Mischzustände) einnehmen 


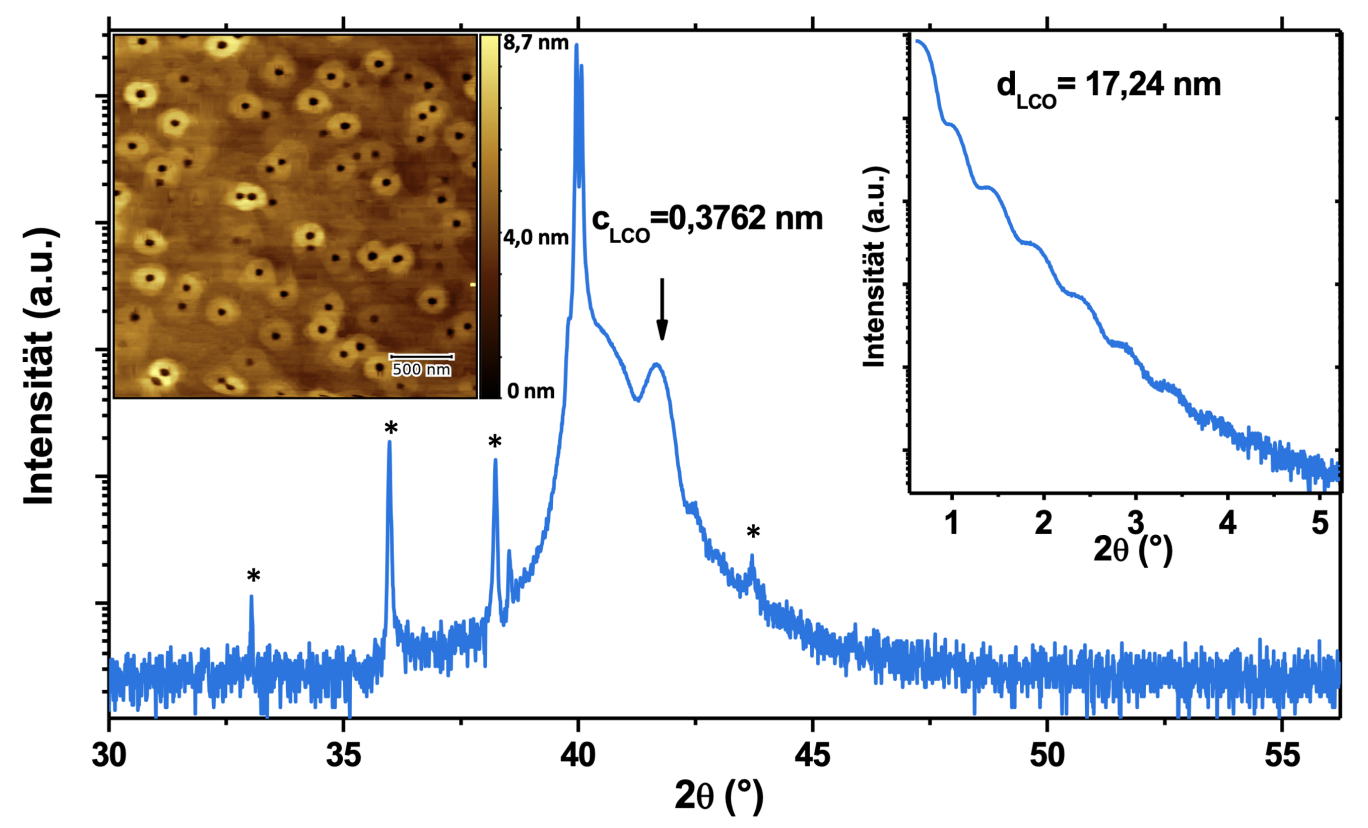

Abbildung 5.14.: XRD-Messung der $\mathrm{LaCoO}_{3}$-Einzelschicht auf STO(111). Im Inlet links ist die AFM-Aufnahme der Probe zu sehen. Im rechten Inlet die dazugehörige XRR-Messung zur Bestimmung der Schichtdicke. Mit * markierte Reflexe sind Messartefakte der Röntgenanlage.

kann. Das Verhalten hängt dabei empfindlich von dem Co-O-Co Winkel ab, der in Dünnfilmen über den epitaktischen Spannungszustand beeinflusst werden kann [37].

Nach Optimierung der Oberfächenmorphologie ergibt sich für das molare La/Co-Verhältnis der Präkursorlösung ein Wert von 1,37. Die Schichtdicke der Einstellschicht beträgt $d_{L C O}=17,24 \mathrm{~nm}$, woraus sich eine Depositionsmenge von $V_{L C O}=3,82 \mu \mathrm{l} /$ u.c. für eine Einheitszelle ergibt. Die Depositionsmenge für $\mathrm{LaMnO}_{3}$ beträgt vergleichbare $V_{L M O}=3,8 \mu \mathrm{l} /$ u.c. Abbildung 5.14 zeigt die Ergebnisse der Röntgenuntersuchungen und im Inlet die AFMAufnahme der Oberflächenmorphologie der $\mathrm{LaCoO}_{3}$-Probe auf STO(111). Aufgrund der Zugspannung auf dem STO-Substrat ${ }^{6}$, fällt die out-of-plane Gitterkonstante mit $c_{L C O}=0,3762 \mathrm{~nm}$ kleiner aus als die pseudokubische Gitterkonstante der Volumenproben [37]. Auffällig sind die Donat-ähnlichen Strukturen der Oberfläche, die sich auch durch weitere Optimierungsversu-

$\overline{{ }^{6} \epsilon=\left(a_{S}-a_{p}\right) / a_{p}=2,63 \% \text { mit }} a_{s}=0,3905 \mathrm{~nm}$ der Gitterkonstante des STO und $a_{p}=0,38 \mathrm{~nm}$ der pseudokubischen Gitterkonstante des Bulk $\mathrm{LaCoO}_{3}$ [37]. 
che nicht vermeiden lassen. Dabei handelt es sich allerdings um einen Effekt, der erst ab einer gewissen Schichtdicke zum Tragen kommt und für die Lagendeposition von nur einer Einheitszelle vernachlässigt werden kann. Die Bereiche zwischen den Punkten zeigen eine glatte Oberfläche auf atomarer Ebene.

Die magnetischen Untersuchungen in Abbildung A.6 im Anhang zeigen ferromagnetisches Verhalten unterhalb von $T_{C}=94 K$ mit einer Sättigung von $M_{\text {sat }}=0,99 \mu_{B} /$ Co. Damit liegt die Übergangstemperatur höher als die der Proben, die mit der PLD hergestellt werden $\left(T_{C, P L D}=85 \mathrm{~K}\right)$ [37], was vermutlich auf den Sauerstoffpartialdruck beim Wachstum in der MAD zurückzuführen ist.

Einfluss der Lagenstöchiometie auf das Probensystem Ähnlich wie im vorhergehenden Kapitel für $\mathrm{LaMnO}_{3} / \mathrm{LaNiO}_{3}$ wird die kontrollierte Herstellung der B-Platzordnung durch abwechselndes Deponieren einer Monolage der Einzelschichtsysteme $\mathrm{LaCoO}_{3}$ und $\mathrm{LaMnO}_{3}$ erzeugt. Die zu deponierende Lösungsmenge ergibt sich hier jedoch nicht aus der kontinuierlichen Reduzierung der Einzelschichtsysteme, sondern direkt aus dem ermittelten Depositionsvolumen für eine Einheitszelle aus den Einstellschichten, $V_{L C O}=3,82 \mu l /$ u.c. und $V_{L M O}=3,8 \mu l /$ u.c. Die Pause zwischen den jeweiligen Depositionen beträgt zwei Sekunden und es werden jeweils 200 Lagen deponiert, woraus sich eine Schichtdicke von $d \approx 88 \mathrm{~nm}$ ergibt.

Zusätzlich wird eine SoA-Referenzprobe hergestellt, in der die Schicht aus einer Lösung mit den drei beteiligten Präkusoren entsteht. Diese zeigt eine Gitterkonstante von $c_{S o A}=0,387 \mathrm{~nm}$ und einen Überstrukturpeak bei $2 \Theta=19,9^{\circ}$, wie in Abbildung 5.15 in Blau zu sehen ist. Aus der Sättigungsmagnetisierung (Abbildung 5.16, Blau) $M_{\text {sat }, \text { SoA }}=5,3 \mu_{B} /(\mathrm{Mn}+\mathrm{Co})$ ergibt sich eine B-Platzordnung von ca. $88 \%$, die jedoch durch weitere Optimierung der Wachstumsbedinungen verbessert werden kann [62].

Die Abbildungen 5.15 und 5.16 zeigen in Rot die Ergebnisse der XRD- und Magnetisierungsmessung der ersten LbL-Probe (L1), $\left(\mathrm{LaMnO}_{3} / \mathrm{LaCoO}_{3}\right)_{200}$. Es ergibt sich eine Gitterkonstante $c_{L 1}=0,383 \mathrm{~nm}$ und die Sättigungsmagnetisierung von $M_{s a t, L 1}=4,1 \mu_{B} /(\mathrm{Mn}+\mathrm{Co})$. Beide Werte weichen gegenüber der SoA deutlich ab. Betrachtet man die Gitterkonstante und vergleicht 


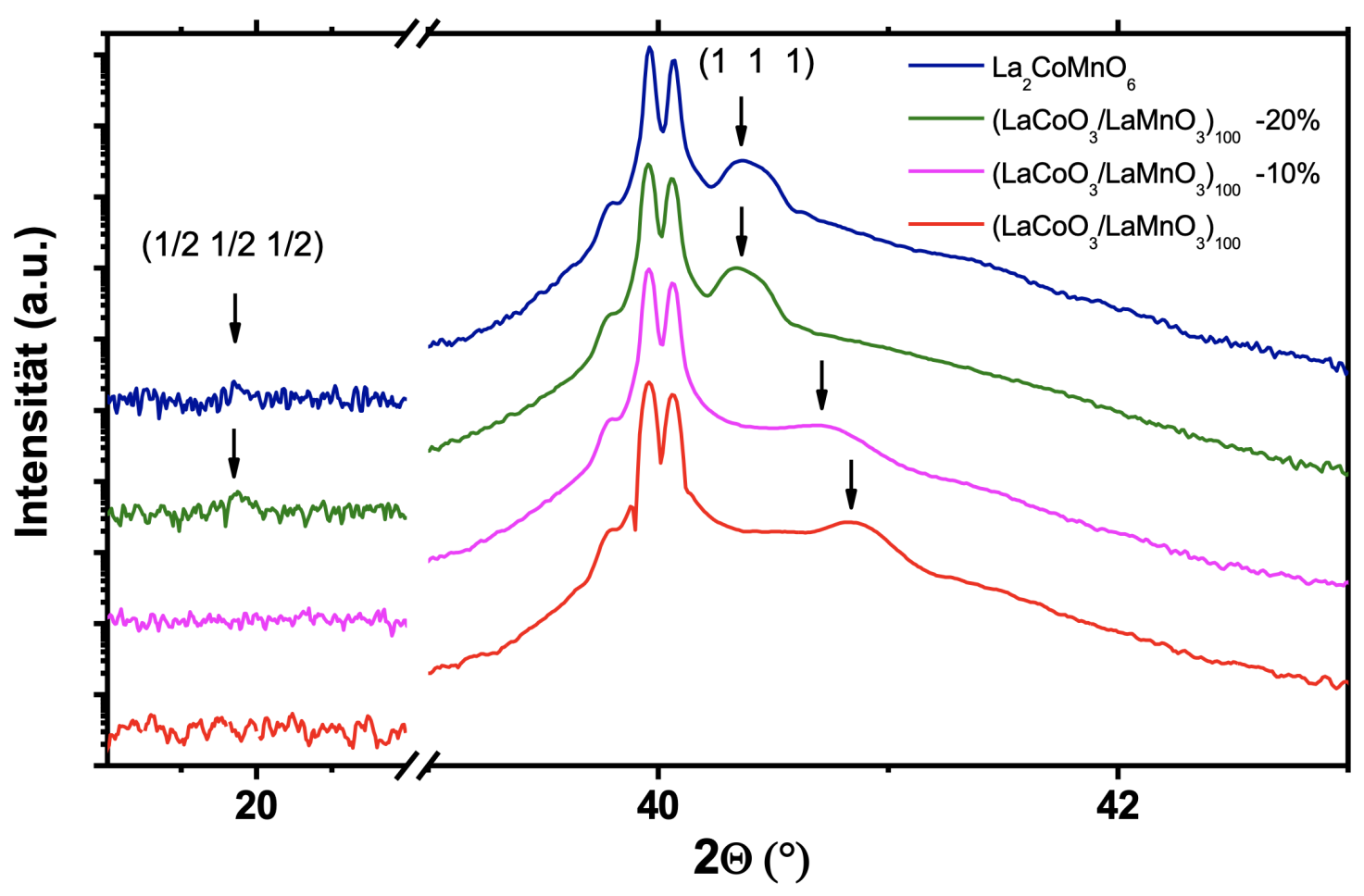

Abbildung 5.15.: XRD-Messungen der SoA-Referenzprobe und der LbLProben mit unterschiedlichen Depositionsmengen für $\mathrm{LaCoO}_{3}$, während die Depositionsmenge für $\mathrm{LaMnO}_{3}$ konstant bleibt. Für die SoA-Probe und L3-Probe ist zusätzlich der Zoom-In auf den Überstrukturpeak dargestellt. Mir Erlaubnis entnommen aus [63].

diese mit den Konstanten der Einzelschichtsysteme, $c_{L M O} \approx 0,3905 \mathrm{~nm}$ und $c_{L C O}=0,3762 \mathrm{~nm}$, und der SoA-Probe, $c_{S o A}=0,387 \mathrm{~nm}$, erkennt man eine Verschiebung der Gitterkonstanten der L1-Probe zum Einzelschichtsystem $\mathrm{LaCoO}_{3}$. Diese Beobachtung führt zu der Vermutung, dass eine erhöhte $\mathrm{LaCoO}_{3}$-Phase vorliegt, die auf ein fehlerhaftes stöchiometrisches Verhältnis der $\mathrm{LaCoO}_{3} \mathrm{zu} \mathrm{LaMnO}$ zurückzuführen ist $(\mathrm{Co} / \mathrm{Mn}>1)$ und zusätzlich die erniedrigte Magnetisierung erklären würde [63].

Um dies zu untersuchen, werden zwei weitere Proben L2 und L3 hergestellt und untersucht, in dem die Depositionsmenge $V_{L C O}$ um jeweils $10 \%$ $\left(V_{L C O, L 2}=3,44 \mu \mathrm{l} /\right.$ u.c. $)$ und $20 \%\left(V_{L C O, L 3}=3,02 \mu \mathrm{l} /\right.$ u.c. $)$ reduziert wird, während die Menge für $\mathrm{LaMnO}_{3}$ konstant bleibt. Die Ergebnisse der Untersuchungen sind den Abbildungen in Magenta (L2) und Grün (L3) beigefügt. 
Die Auswirkungen der Reduzierung von $V_{L C O}$ und der damit einhergehenden stöchiometrischen Änderung des Lagenverhälnisses $\left(\mathrm{LaMnO}_{3} / \mathrm{LaCoO}_{3}\right)$ zeigen einen direkten Einfluss auf die strukturellen Eigenschaften der Schichten. Mit abnehmender Depositionsmenge für $\mathrm{LaCoO}_{3}$ verschiebt sich die Gitterkonstante in Richtung des (111)-Peaks der SoA-Probe, wie Abbildung $5.15 \mathrm{zu}$ entnehmen ist. Dieses wirkt sich auch auf die Magnetisierungsmessungen aus, die mit den strukturellen Eigenschaften korrelieren: je kleiner der Unterschied der Gitterkonstanten der LbL-Probe zu der SoA-Probe, desto höher ist die Sättigungsmagnetisierung und die Übergangstemperatur. So führt die Reduzierung des Depositionsvolumen für $\mathrm{LaCoO}_{3}$ bei der L2-Probe zu einer Erhöhung der Sättigungsmagnetisierung auf $M_{s a t, L 2}=$
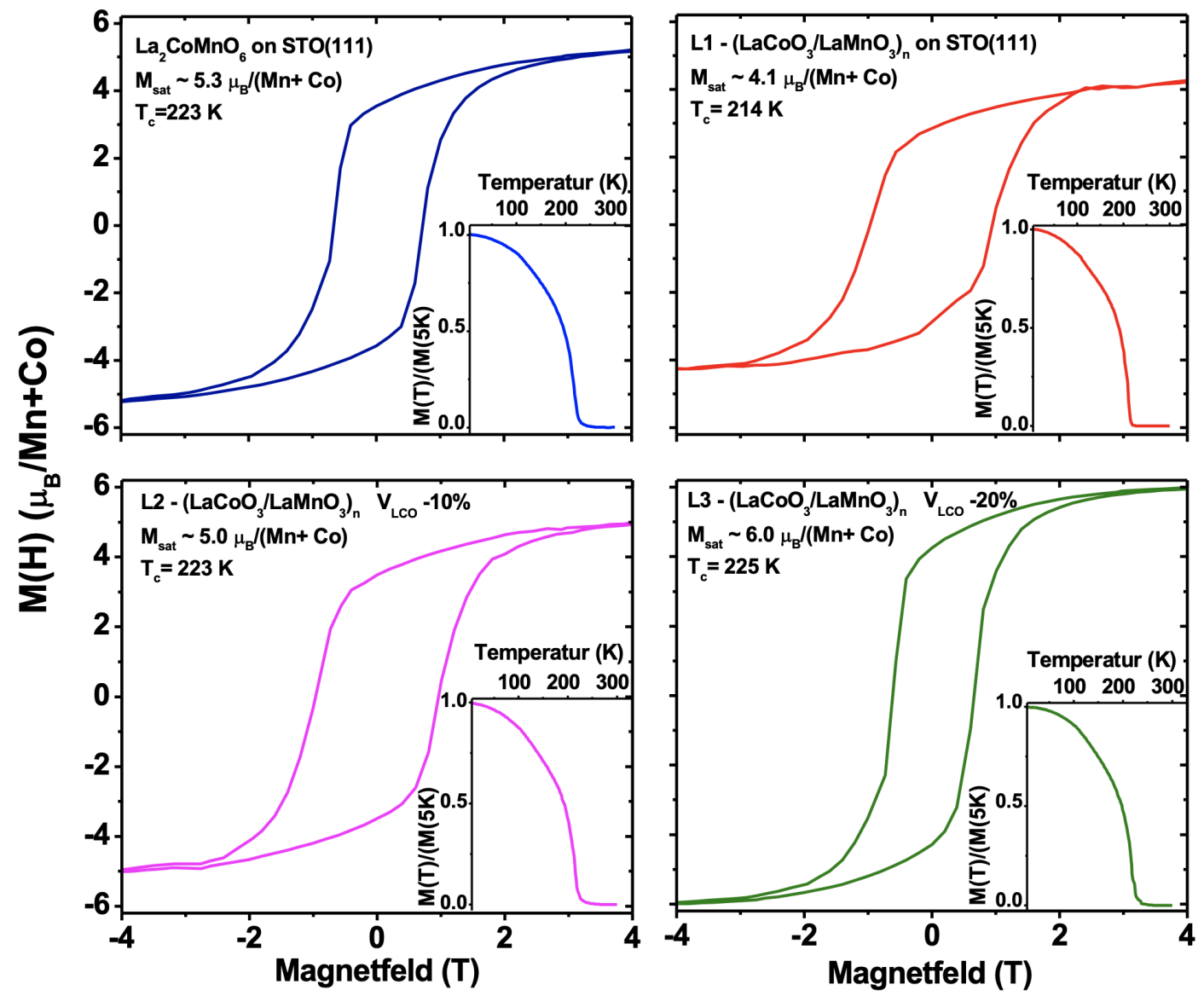

Abbildung 5.16.: SQUID-Messungen der SoA- und LbL.Proben. Die temperaturabhängige Messung wurden bei $H=1000$ Oe aufgenommen und die feldabhängige Messung bei $T=5 \mathrm{~K}$. Mit Erlaubnis entnommen aus [63]. 
$5,0 \mu_{B} /(\mathrm{Co}+\mathrm{Mn})$. Die Gitterkonstante beträgt $c_{L 2}=0,3861 \mathrm{~nm}$. Die resultierenden Eigenschaften nähern sich den Eigenschaften der SoA-Probe somit weiter an, wenn auch gleich diese noch nicht vollständig übereinstimmen. Dieser Trend setzt sich bei weiterer Reduzierung für die L3-Probe weiter fort, die bemerkenswerte Eigenschaften aufweist. Die Gitterkonstante entspricht mit $c_{L 3}=0,387 \mathrm{~nm}$ der Gitterkonstanten der SoA-Probe, was sich auch in den magnetischen Eigenschaften niederschlägt. Die Übergangstemperatur erhöht sich auf $T_{C, L 3}=225 \mathrm{~K}$ und die Sättigungsmagnetisierung erreicht erstmal das theoretische Maximum mit $M_{s a t, L 3}=6 \mu_{B} /(\mathrm{Co}+\mathrm{Mn})$, die auf eine noch nie erreichte B-Platzordnung von bis zu $100 \%$ hindeutet. Zusätzlich zeigt die Hysterese eine vergleichbar kleine Koerzitivfeldstärke mit $H_{c} \approx 6 k O e$, die auf große ferromagnetische Domänen mit hoher BPlatzordnung deutet $[67,73]$. Dies zeigt, dass die Ausbildung von antiferromagnetischen Kopplungen durch Antiphasengrenzen unterdrückt sein muss. Zudem wird der (1/2 1/2 1/2)-Überstrukturpeak beobachtet.

Um die Bestimmung des hohen Grades der B-Platzordnung weiter zu bestätigen, werden TEM-Untersuchungen an der L3-Probe durchgeführt, die in Abbildung $5.17 \mathrm{zu}$ sehen sind. Die Abbildung zeigt einen homogen gewachsenen und defektfreien Film mit einer Schichtdicke von $d \approx 80 \mathrm{~nm}$, die bei 200 Einzellagenwiederholungen zu erwarten ist. Es besteht eine atomar glatte, kohärente Grenzfläche zwischen Substrat und Film. Die EELSMessung im Inlet zeigt, dass sich in (111)-Richtung die unterschiedlichen Lagen gemäß der B-Platzordnung abwechseln. Dabei sind die Mn-Kationen Grün und die Co Kationen Rot dargestellt. Jedoch lassen sich doch auch farbliche Variationen innerhalb einer Lage erkennen, die darauf hinweisen, dass keine vollständige $\mathrm{Co} / \mathrm{Mn}$-Ordnung erreicht wird. Es ist jedoch nicht ausgeschlossen, dass diese lokale Unordnung bei der Herstellung der TEMLamelle entstand.

Der Einfluss der Depositionsmenge für $\mathrm{LaCoO}_{3}$ soll tiefer untersucht werden, in dem weitere Proben mit $V_{L C O}=3,62 \mu l / u . c ., 3,30 \mu l / u . c ., 3,20 \mu l /$ u.c. und 3,10 $\mu \mathrm{l} /$ u.c. hergestellt werden. Die ermittelten Werte für die Sättigungsmagnetisierungen und die Gitterkonstanten sind in Abbildung 5.18 zusammengefasst. Die Abbildung lässt eine Korrelation zwischen der De- 


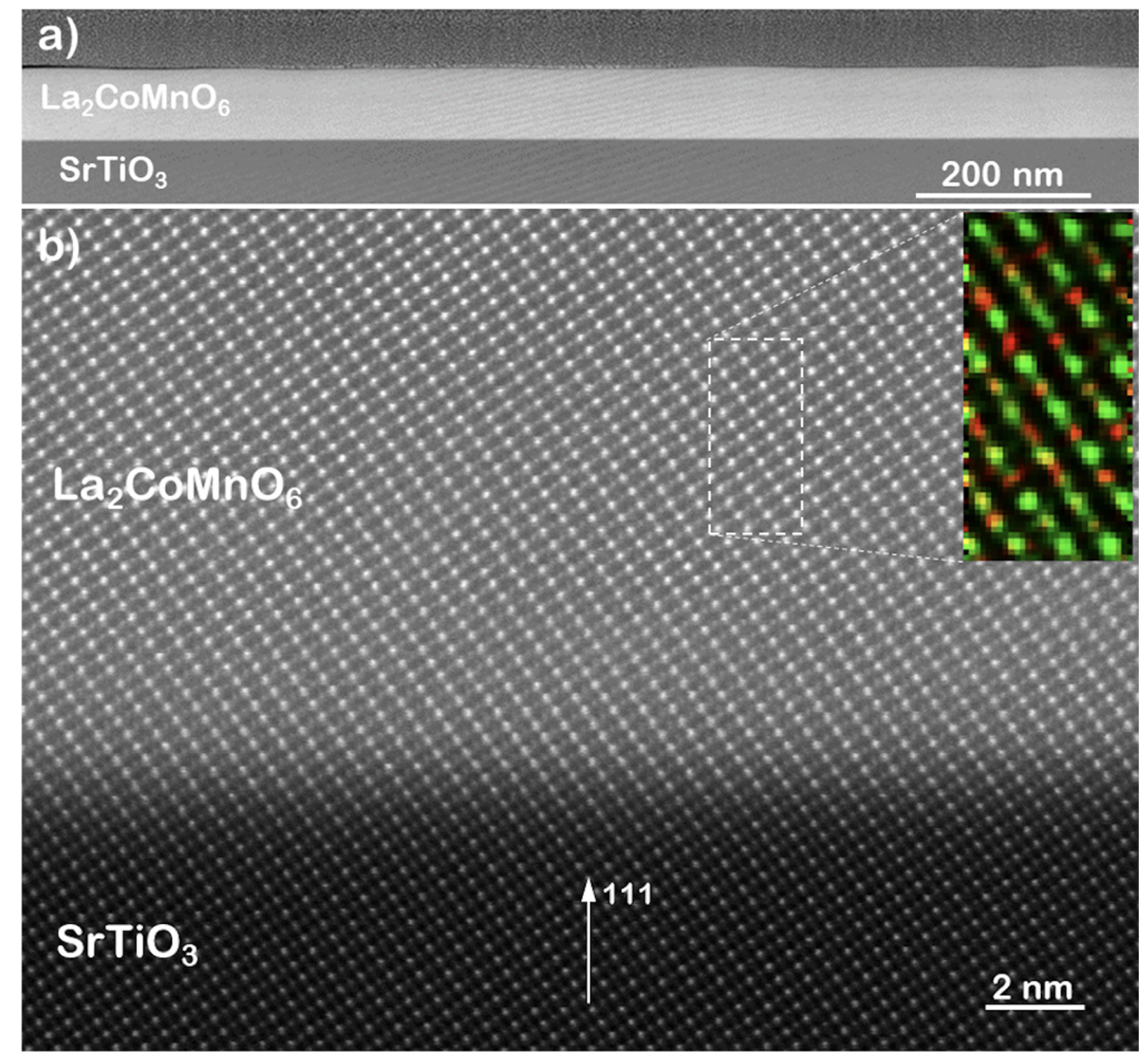

Abbildung 5.17.: a) HAADF STEM-Aufnahme mit b) einer Nahaufnahme der L3-Probe mit c) EELS-Mapping. Angefertigt von Vladimir Roddatis am Helmholtz-Zentrum in Potsdam. Mit Erlaubnis entnommen aus [63].

positionsmenge und den strukturellen und magnetischen Eigenschaften erkennen, die sich bei Reduzierung der Depositionsmenge für $\mathrm{LaCoO}_{3}$ kontinuierlich verändert. Im Bereich von 3,30 $\mu \mathrm{l} /$ u.c. $<V_{L C O}<3,82 \mu \mathrm{l} /$ u.c. besteht ein nahezu linearer Zusammenhang zwischen der Depositionsmenge und den jeweiligen Eigenschaften [63].

Zusammenfassung und Diskussion Die vorgestellten Ergebnisse zeigen eindrucksvoll, dass die B-Platzordnung für $\mathrm{La}_{2} \mathrm{CoMnO}_{6}$ durch das Lagenwachstum weiter optimiert werden kann. Ermöglicht wird dies durch das optimierte Wachstum der Einzelschichtsysteme $\mathrm{LaCoO}_{3}$ und $\mathrm{LaMnO}_{3}$ innerhalb der MAD. Das Aufwachsen der einzelnen Schichten führt für jede 


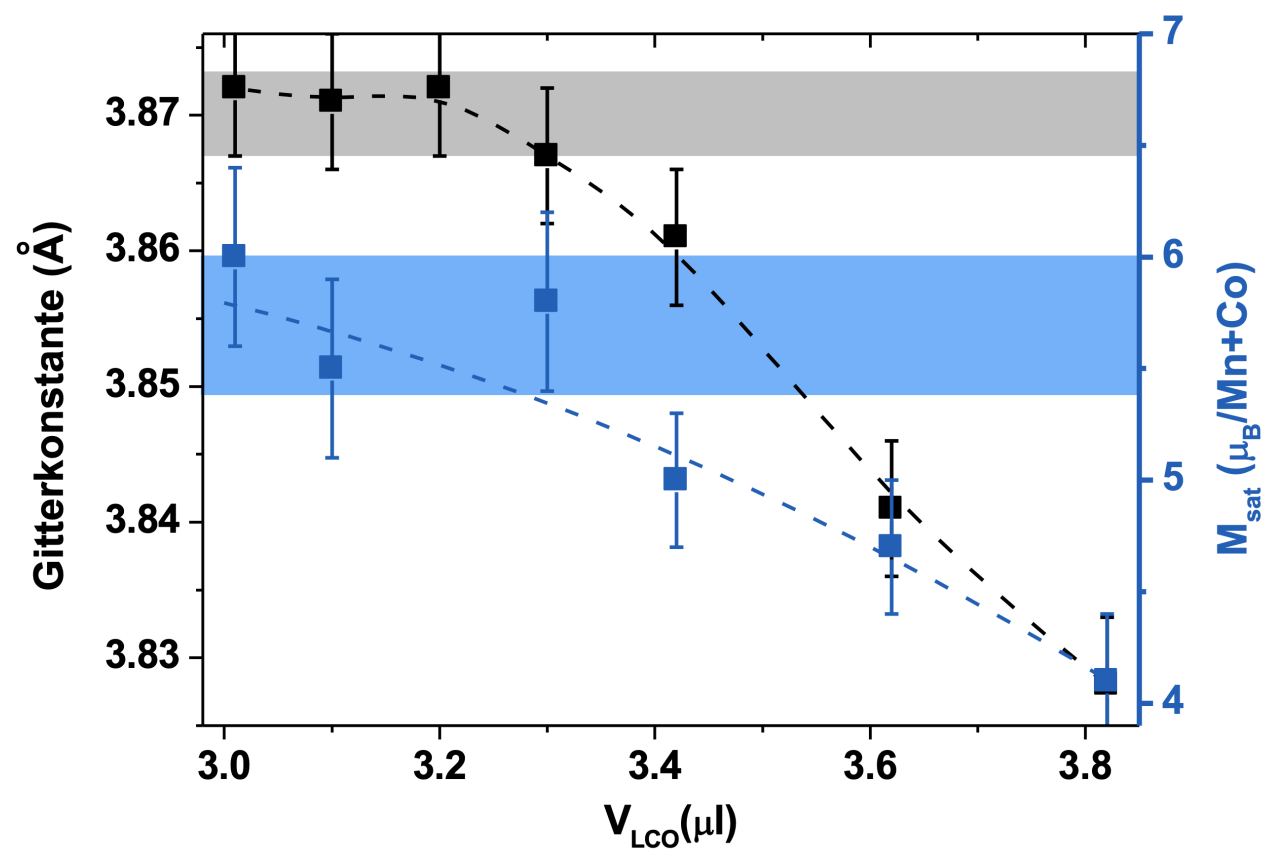

Abbildung 5.18.: Einfluss der Depositionsmenge des $\mathrm{LaCoO}_{3}$ auf die Gitterkonstante und Sättigungsmagnetisierung. In Grau und Blau sind die Bereiche hinterlegt, die für die SoA-Proben erwartet werden und in denen sich eine Stabilisierung der Werte in Abhängigkeit von der Depositionsmenge einstellt. Entnommen aus [63].

Lage zu einem geschlossenen Film, der die Bildung von Defekten zu unterdrücken scheint. Magnetisierungsmessungen deuten auf eine vollständige B-Platzordnung hin, wohingegen unklar bleibt, weshalb der Überstrukturpeak nur schwach ausgeprägt ist. Wie bereits erwähnt, kann dies eine Vielzahl an Ursachen haben. Es wird aber bisher oftmals mit APB begründet. Antiphasengrenzen sollten jedoch mittels des Lagenwachstums ausgeschlossen werden. Die Ursache dafür muss somit weiter untersucht werden und scheint von einer nicht trivialen Natur zu sein. Ansatzpunkt für die Untersuchung des Überstrukturpeaks bildet dabei die Doppelperowskitreihe $\mathrm{A}_{2} \mathrm{CoMnO}_{6}$ mit $\mathrm{A}=\mathrm{La}, \mathrm{Pr}, \mathrm{Nd}, \mathrm{Sm}, \mathrm{Gd}$ und $\mathrm{Y}$, die im Rahmen der Promotionsarbeit von Christoph Meyer [62] untersucht wurden. Während auch dort für La ein ungewöhnlich kleiner Peak beobachtet wird, wird hingegen für $\mathrm{Nd}$ ein unerwartet großer Überstrukturpeak beobachtet. Es scheint ein Zusammenhang zwischen der Größe des A-Platzes und der daraus resultie- 
renden Struktur mit bestimmten Abstandsmodelationen zu geben, die den Peak ausschlaggebend beeinflussen. Diese werden scheinbar über die theoretische Berechnung mit Hilfe der Strukturfaktoren nicht betrachtet. Genaue TEM-Untersuchungen können dabei Aufschluss über diese Beobachtung liefern.

Aus diesem Beispiel heraus stellt sich allgemein die Frage, wie man zuverlässig und reproduzierbar den Grad der B-Platzordnung quantifizieren kann, da die einzelnen Untersuchungsmöglichkeiten jeweils nur Aufschluss über die langreichweitige (Raman, XRD) oder kurzreichweitige (TEM, EELS) Kationordnung liefern und nicht immer miteinander übereinstimmen müssen. Diese Frage lässt sich nicht pauschal beantworten und muss für jedes Materialsystem neu beantwortet werden. Beispielsweise eignen sich für das vorgestellte System $\mathrm{La}_{2} \mathrm{CoMnO}_{6}$ die Magnetisierungsmessungen, da das theoretische maximale Moment bekannt ist. Es ist natürlich klar, dass ein solches Verfahren nicht auf nicht ferromagnetische Proben anwendbar ist, wie das kommende Beispiel $\mathrm{Sr}_{2} \mathrm{TiRuO}_{6}$ noch zeigen wird.

Die Variation des Verhältnisses der Depositionsmengen für $\mathrm{LaCoO}_{3}$ und $\mathrm{LaMnO}_{3}$ zeigt eine eindeutige Korrelation mit den magnetischen und strukturellen Eigenschaften der Schicht. Bereits bei geringen Abweichungen der Einzelschichtdicke für das $\mathrm{LaCoO}_{3}$ größer als eine Einheitszelle $\left(d_{L C O}>\right.$ 1 u.c.) bildet es seine Eigenschaften als Einzelschichtsystem aus und überträgt die strukturellen Eigenschaften auf das gesamte Probensystem, weshalb sich die Gitterkonstante mit zunehmender Depositionsmenge $V_{L C O}$ in Richtung des $\mathrm{LaCoO}_{3}$ Peaks bewegt. Diese Beobachtung kann sich für zukünftige Untersuchungen als sehr hilfreich erweisen, wenn auf ähnliche Weise künstlich die B-Platzordnung erzeugt werden soll. In Abhängigkeit der Gitterkonstanten kann so das passende stöchiometrische Verhältnis der zu deponierenden Lösungsmenge nach bereits einer Probe abgeschätzt werden. Diese Erkenntnis soll im folgenden Kapitel an einem weiteren Materialsystem näher erprobt werden und so auch die Fragestellung klären, inwiefern sich dieses Verfahren auf andere Materialsysteme und Depositionsmethoden übertragen lässt. 


\subsection{2. $\mathrm{Sr}_{2} \mathrm{TiRuO}_{6}$ und $\mathrm{SrTiO}_{3} / \mathrm{SrRuO}_{3}-$ Heterostrukturen}

Motiviert durch diese Ergebnisse, sollen die Anwendungsmöglichkeiten der Herstellung synthetischer B-Platzordnung mittels Lagenwachstum weiter erprobt und das Verständnis der B-Platzordnung vertieft werden.

Dafür wird das vorgestellte Verfahren auf das $\mathrm{DP} \mathrm{Sr}_{2} \mathrm{TiRuO}_{6}$ angewandt. Die Proben werden nach dem selben Prinzip hergestellt, wie im Kapitel zuvor: Neben einer SoA-Probe wird in einem ersten Schritt eine LbL - Probe auf STO(111) hergestellt. Die Depositionstemperatur beträgt auch hier $950^{\circ}$. Die Wachstumsbedingungen für die Einzelschichtsysteme $\mathrm{SrRuO}_{3}$ und $\mathrm{SrTiO}_{3}$ werden zuvor bestimmt und sollen auch hier vorgestellt werden.

Einzelschichtsysteme $\mathrm{SrRuO}_{3}$ und $\mathrm{SrTiO}_{3}$ Die Wachstumsbedingungen für $\mathrm{SrTiO}_{3}$ werden an die MAD mit Hilfe der in-situ-Ellipsometrie optimiert. Dabei wird das $\mathrm{SrTiO}_{3}$ auf selbiges $\mathrm{SrTiO}_{3}$-Substrat deponiert. Sofern die stöchiometrische Zusammensetzung der Lösung und der daraus wachsenden Schicht mit der des Substrates übereinstimmt, sollte sich das Ellipsometriesignal nicht verändern. Auf diese Weise wurde im Rahmen der Masterarbeit von Vitaly Bruchmann-Bamberg ein $\mathrm{Sr} / \mathrm{Ti}$ Verhältnis von 0,78 in der Lösung bestimmt, das auch hier verwendet wird [169]. Die Abhängigkeit des Ellipsometriesignales von dem Ti/Sr-Verhältnisses ist in Abbildung A.7 im Anhang zu sehen ${ }^{7}$. Aus der Herstellung der $\mathrm{SrTiO}_{3}$-Einzelschichten ergibt sich eine Depositionsmenge von $V_{S T O}=9,33 \mu l / u . c$. für eine Einheitszelle.

$\mathrm{SrRuO}_{3}$ zeigt ferromagnetisches Verhalten und eine metallische Leitfähigkeit. Zur Optimierung der Wachstumsbedingungen wird das Restwiderstandsverhältnis (RRR, engl.: residual resistance ratio) $R(300 \mathrm{~K}) / R(5 \mathrm{~K})$ maximiert. Die Untersuchungen fanden im Rahmen der Bachelorarbeit von Leonard Schüler statt und können in seiner Arbeit genau nachvollzogen werden [170]. Dabei hat sich herausgestellt, dass $\mathrm{SrRuO}_{3}$ besonders glatt aufwächst, wenn die Depositionsgeschwindigkeit im Vergleich zu den bisherigen Materialsystemen mit $r_{M A D}$ um ein Vielfaches höher ist, $r_{S R O}=$

\footnotetext{
${ }^{7}$ Mit Erlaubnis entnommen aus der Masterarbeit von Vitaly Bruchmann-Bamberg [169].
} 
$0,18 \mathrm{ml} / \mathrm{s}=4,5 \times r_{M A D}$. Die $\mathrm{SrRuO}_{3}$ Dünnfilme zeigen eine out-of-plane Gitterkonstante von $c_{S R O}=0,395 \mathrm{~nm}$. Es wird eine Depositionsmenge von $V_{S R O}=2,0 \mu l /$ u.c. für eine Einheitszelle benötigt bei einem $\mathrm{Sr} / \mathrm{Ru}-$ Verhältnis von 1,2 .

$\mathrm{Sr}_{2} \mathrm{TiRuO}_{6} \quad \mathrm{Sr}_{2} \mathrm{TiRuO}_{6}$-Dünnfilme, die mittels PLD hergestellt werden [171], zeigen unabhängig von der B-Platzordnung isolierendes Verhalten und kein magnetisches Moment, das auf Ferromagnetismus zurückzuführen ist. So auch hier: die State-of-Art-Probe, die aus einer Lösung in der MAD hergestellt wird, zeigt kein magnetisches Moment und weist isolierendes Verhalten auf, wie in Abbildung 5.24 in Schwarz im nächsten Abschnitt zu sehen ist. Die Röntgenuntersuchung ergibt einen ausgeprägten $\left(1 / 2^{1 / 2} 1 / 2\right)$-Überstrukturpeak (siehe Abbildung 5.19) bei $2 \Theta=19,65^{\circ}$, aus dem sich für das Intensitätsverhältnis zum (111)-Peak ein Ordnungswert

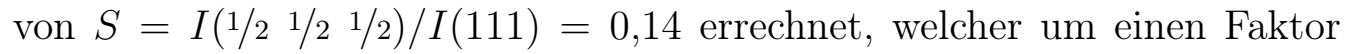
zwei von dem theoretischen Wert $S_{\text {Theo }}=0,3$ abweicht [171].

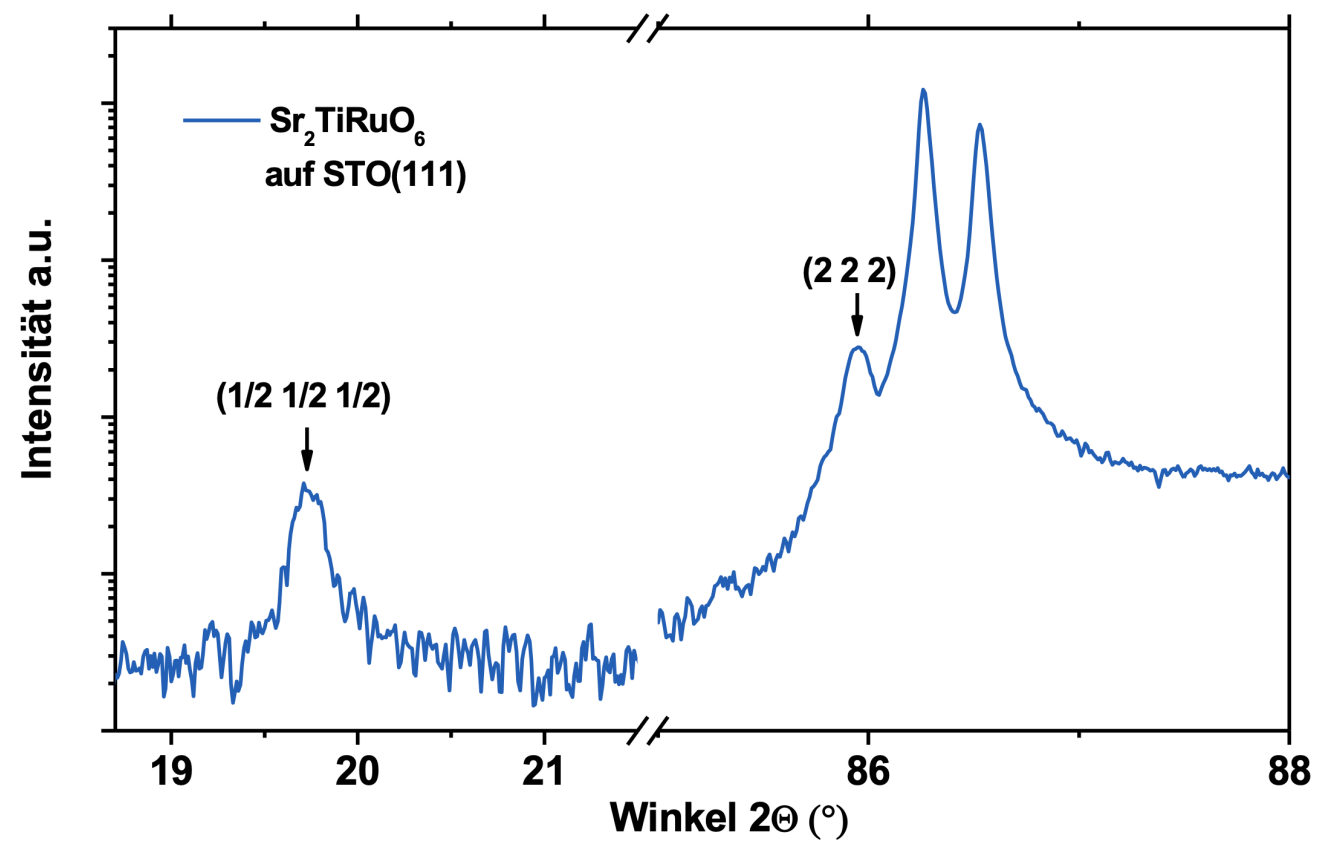

Abbildung 5.19.: Weitwinkelmessungen der $\mathrm{Sr}_{2} \mathrm{TiRuO}_{6}$-Referenzprobe auf STO(111), die aus einer Lösung gewachsen wird. 

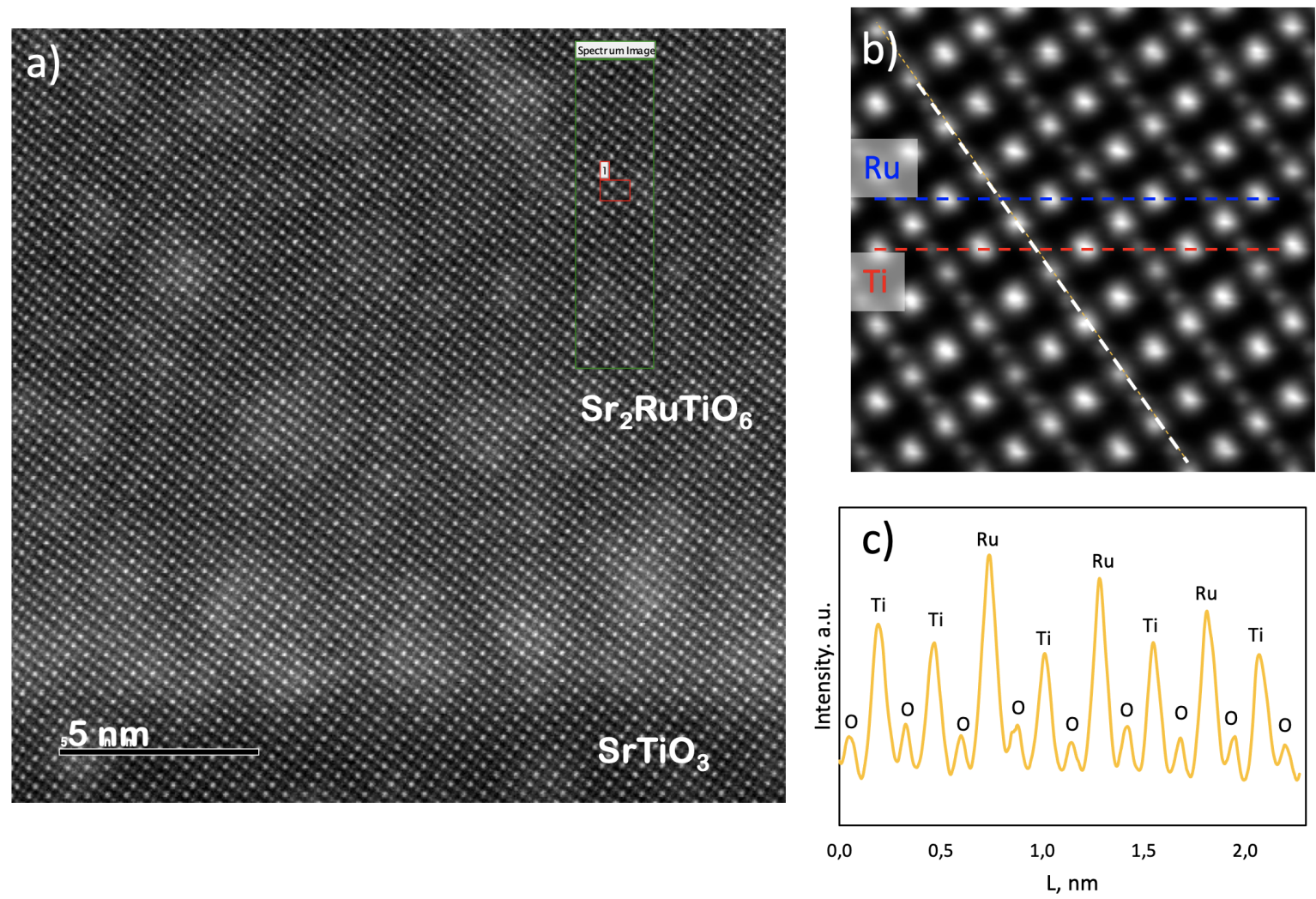

Abbildung 5.20.: a) HAADF STEM-Aufnahme der $\mathrm{Sr}_{2} \mathrm{TiRuO}_{6}$ Probe auf STO(111), b) Nahaufnahme und c) Intensitätsverlauf der Aufnahme entlang der weißen Linie in b) zur Identifizierung des Ti und Ru. Angefertigt von Vladimir Roddatis am Helmholtz-Zentrum in Potsdam.

Um das Auftreten des Überstrukturpeaks und der damit verbundenen BPlatzordnung weiter zu untersuchen, werden TEM-Untersuchungen an der Probe durchgeführt, die in den Abbildungen 5.20 a) und b) zu sehen sind. Da das EELS-Spektrum des Ruthenium von dem des Titans überdeckt wird, lassen sich mit Hilfe des EELS-Mapping keine genauen Aussagen über die BPlatzordnung treffen. Aus dem Grund wird der Intensitätsverlauf zur Analyse der B-Platzordnung herangezogen, da das Ruthenium aufgrund der größeren Dichte eine höhere Intensität als das Titan aufweist, wie in Abbildung 5.20 c) gezeigt ist. Die Abbildung zeigt entlang der (111)-Richtung abwechselnd hohe Intensitäten, die jeweils dem Ruthenium und Titan zuzuordnen sind und damit die B-Platzordnung im System zeigt.

Betrachtet man das Beugungsmuster der Aufnahme im Inlet von Abbil- 

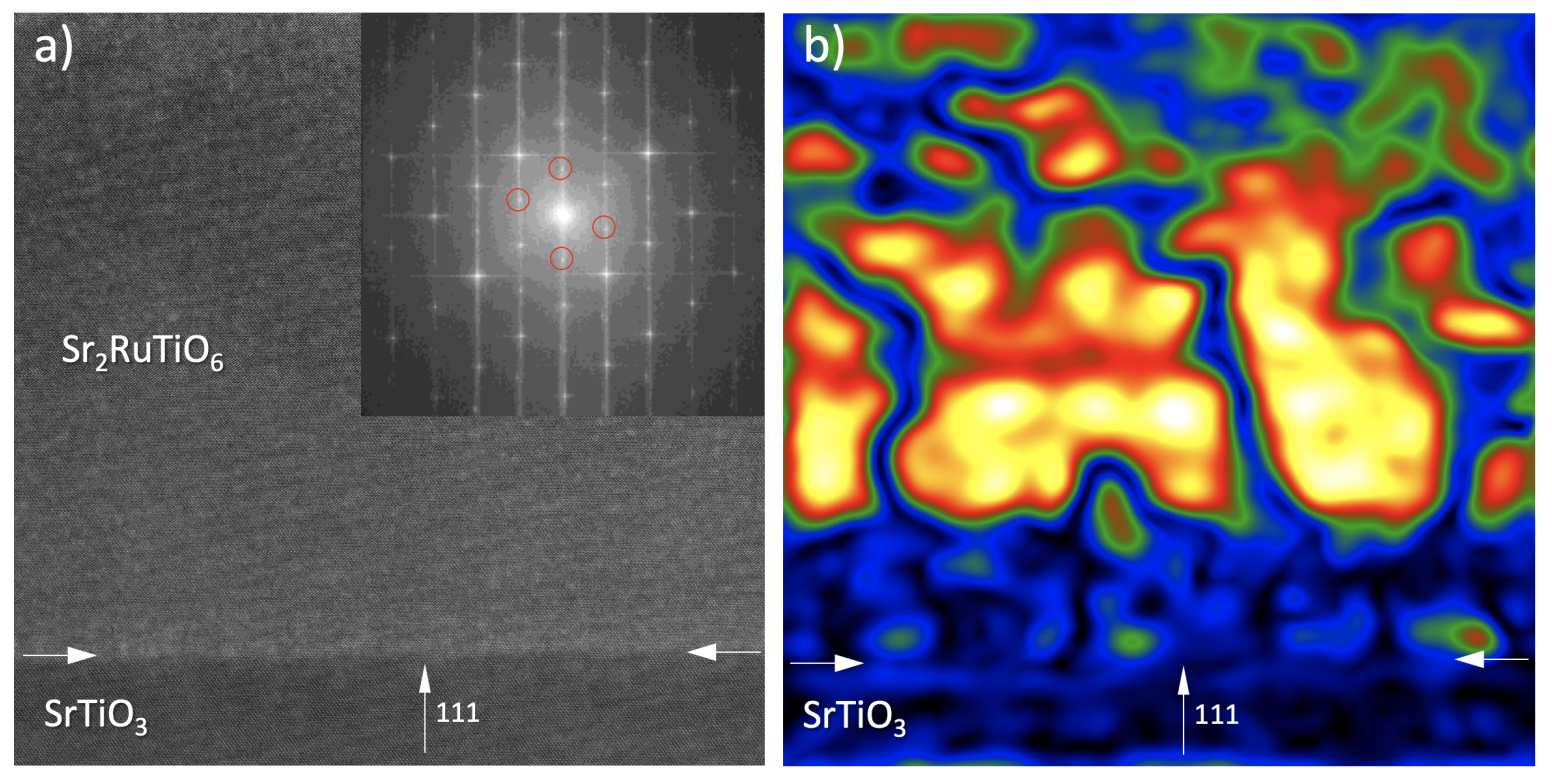

Abbildung 5.21.: a) TEM-Aufnahme der $\mathrm{Sr}_{2} \mathrm{TiRuO}_{6}$ Probe auf STO(111) mit dem Beugungsbild im Inlet, b) Rekonstruktion der Aufnahme aus den Peaks der Überstruktur. Maßstab wie in Abbildung 5.20 a). Angefertigt von Vladimir Roddatis am Helmholtz-Zentrum in Potsdam.

dung $5.21 \mathrm{a}$ ), sind die Peaks der Überstruktur eindeutig zu erkennen (Rot markiert). Um die Bereiche mit hoher B-Platzordnung zu identifizieren, wird aus diesen ausgewählten Punkten die Aufnahme im Realraum rekonstruiert, was in Abbildung 5.21 b) zu sehen ist. Aus der Intensitätsverteilung zeigt sich, dass die Probe Regionen bzw. Domänen aufweist, die eine hohe B-Platzordnung zeigen, die durch schmale Bereiche mit geringer BPlatzordnung voneinander getrennt sind. Die Domänen decken den Großteil der Probe ab. Allerdings zeigt sich auch, dass die Ordnung sich erst nach einer Schichtdicke von ca. $3 \mathrm{~nm}$ in weiten Bereichen ausbreitet und nahe dem Substrat nur in geringem Maße vorliegt.

Um die Probe auf einen Ladungstransfer zwischen $\mathrm{Ru}^{4+}$ und $\mathrm{Ti}^{4+}$ hin $\mathrm{zu}$ untersuchen $^{8}$, werden EELS-Spektren des Titans an der $\mathrm{L}_{2,3}$-Kante und des Sauerstoffes an der K-Kante an unterschiedlichen Orten der Probe aufgenommen. Dabei wird versucht, das erste Spektrum in der $\mathrm{SrTiO}_{3}$-Ebene entlang der (111)-Orientierung aufzunehmen, die in Abbildung 5.20 b) in

\footnotetext{
${ }^{8}$ Ähnlich wie er in den bereits vorgestellten Systemen $\mathrm{La}_{2} \mathrm{NiMnO}_{6}$ und $\mathrm{La}_{2} \mathrm{CoMnO}_{6}$ mit $\mathrm{Mn}^{4+} / \mathrm{Ni}^{2+}$ und $\mathrm{Mn}^{4+} / \mathrm{Co}^{2+}$ existiert.
} 
Rot eingezeichnet ist, das zweite Spektrum in der $\mathrm{SrRuO}_{3}$-Ebene, die in Blau in der Abbildung eingezeichnet ist, und schließlich das dritte Spek-

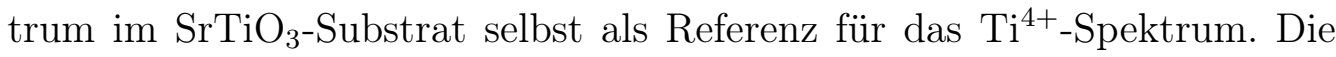
Spektren sind in Abbildung 5.22 a) für Titan und b) für das Sauerstoff zu sehen. Innerhalb des STO-Substrats zeigt sich ein klares Spektrum für die Ti $\mathrm{L}_{2,3}-$ Kante mit vier ausgezeichneten Peaks bei $458 \mathrm{eV}, 461 \mathrm{eV}, 464 \mathrm{eV}$ und $466 \mathrm{eV}$, die allesamt $\mathrm{Ti}^{4+}$ zuzuordnen sind [172]. In der $\mathrm{SrRuO}_{3}$-Ebene (Blau) wird ein ähnlicher Intensitätsverlauf gemessen, jedoch ist der Peak bei $466 \mathrm{eV}$ im Vergleich zum Peak im STO-Substrat abgeschwächt.In der $\mathrm{SrTiO}_{3}$-Ebene zeigt sich hingegen eine deutliche Abweichung vom Spektrum des Substrates. Die Peaks bei $464 \mathrm{eV}$ und $466 \mathrm{eV}$ verschwimmen zu einem breiten gemeinsamen Peak, der dem $\mathrm{Ti}^{3+}$ zuzuordnen ist [172] und somit auf einen Ladungstransfer $\mathrm{Ti}^{4+}+\mathrm{Ru}^{4+} \rightarrow \mathrm{Ti}^{3+}+\mathrm{Ru}^{5+}$ hindeutet. Die Messungen für das Sauerstoff innerhalb der Probe zeigen keine Auffälligkeiten und erlauben aufgrund des hohen Rauschens keine weiteren Rückschlüsse.
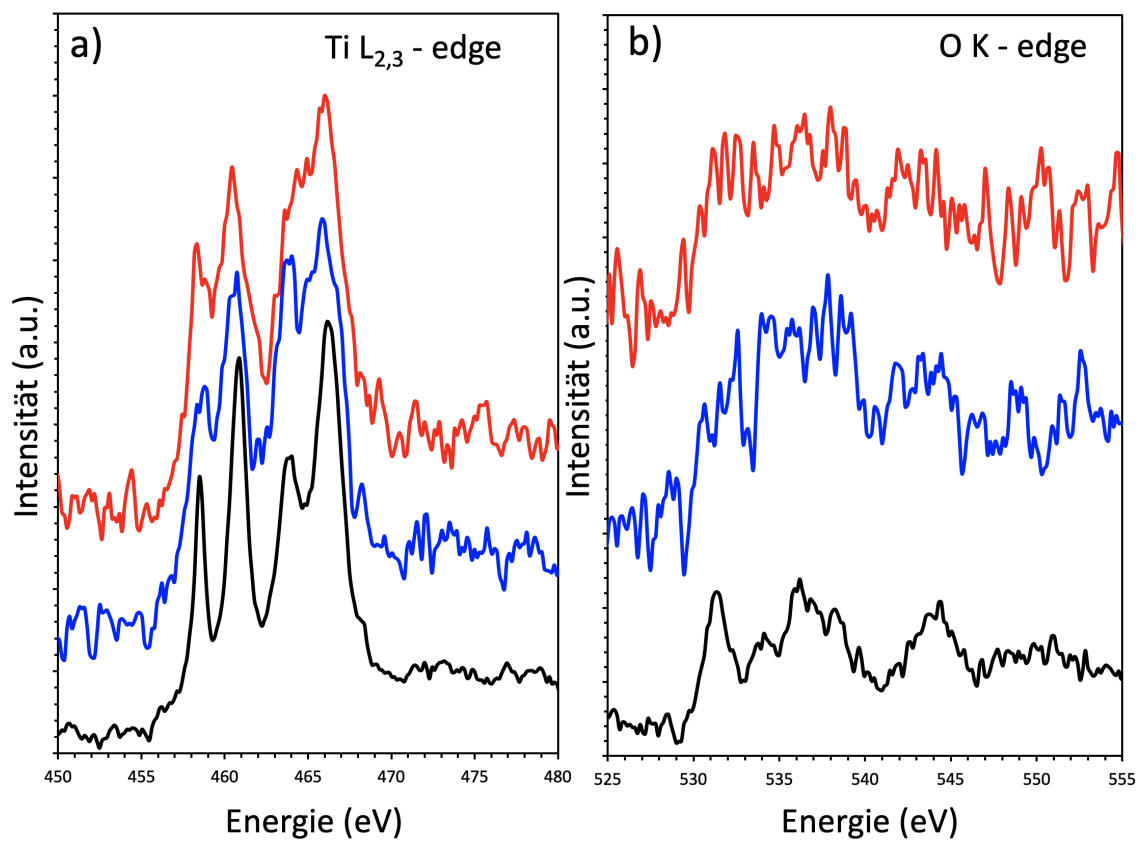

Abbildung 5.22.: a) EELS-Spektrum des Titans und b) des Sauerstoffs. Rot in der Titan-Ebene entlang der (111)-Orientierung, Blau in der Ruthenium-Ebene und schließlich Schwarz als Referenzmessung für $\mathrm{Ti}^{4+}$ im STO-Substrat. Angefertigt von Vladimir Roddatis am Helmholtz-Zentrum Potsdam. 
Einfluss der Lagenstöchiometrie auf $\mathrm{SrTiO}_{3} / \mathrm{SrRuO}_{3}$ Die Untersuchungen an der SoA-Probe haben gezeigt, dass die B-Platzordnung im System existiert, sich jedoch nur innerhalb von Domänen, die durch Regionen mit geringer Ordnung voneinander getrennt sind, ausbildet. Diese Domänen müssen bereits während des Wachstums aus einer Lösung innerhalb der MAD entstehen. Betrachtet man die vorhergehenden Ergebnisse der lagenweise Deposition der einzelnen $\mathrm{LaCoO}_{3}$ - und $\mathrm{LaMnO}_{3}$-Lagen zur Herstellung künstlicher B-Platzordnung und geht davon aus, dass jede Einzellage einen geschlossenen Film bildet, so müsste das Wachstum der Domänen in senkrechter Richtung unterdrückt werden. Dadurch müsste die Ausbildung der B-Platzordnung in der gesamten Probe gleichmäßig ermöglicht werden. Um dies zu untersuchen, wird eine LbL-Probe L1 durch abwechselnde Deposition der Einzelschichtsysteme $\mathrm{SrTiO}_{3}$ und $\mathrm{SrRuO}_{3}$ anhand der vorgestellten Wachstumsbedingungen hergestellt und untersucht. Die Röntgenunter-

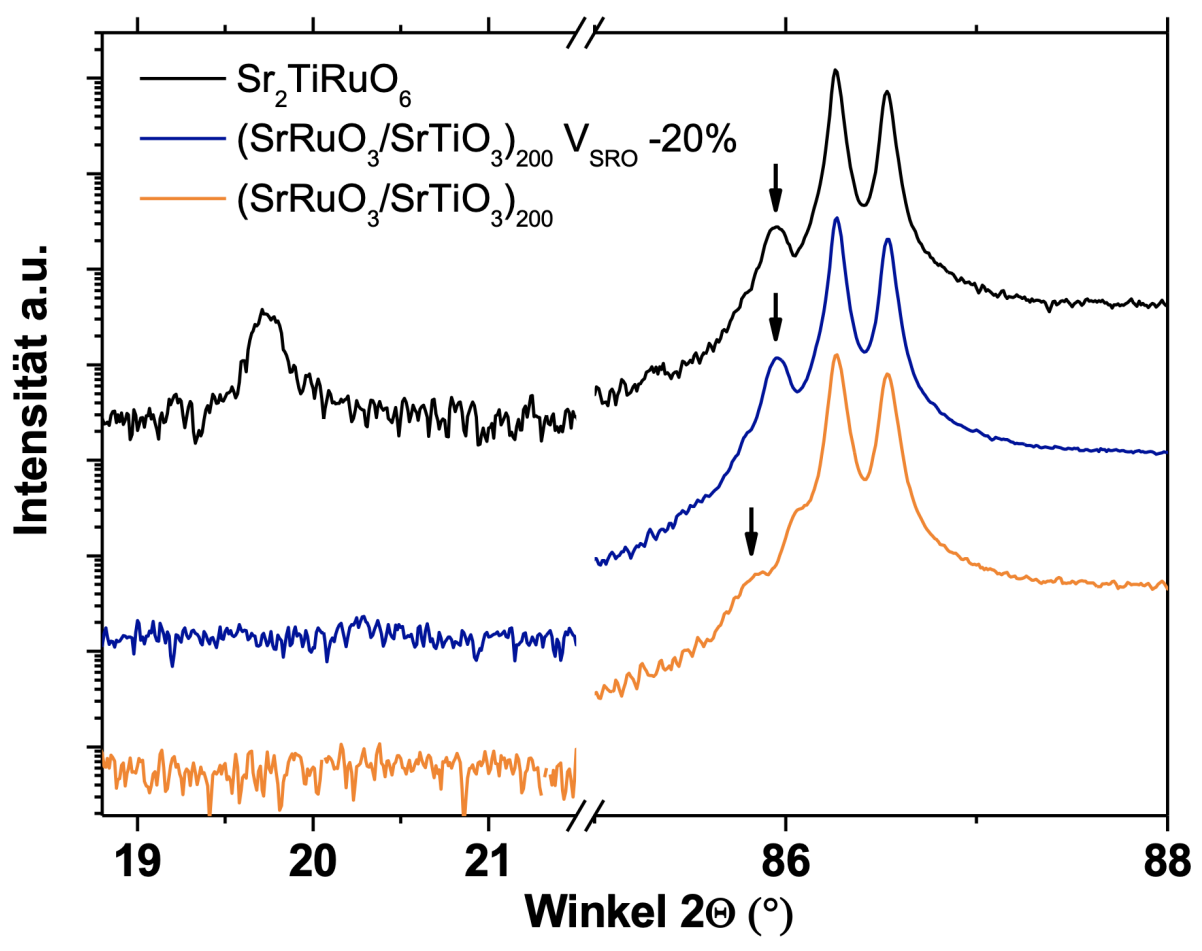

Abbildung 5.23.: Weitwinkelmessungen der $\mathrm{Sr}_{2} \mathrm{TiRuO}_{6}$-Referenzprobe (Schwarz), die aus einer Lösung gewachsen wird, und der lagenweise aufgewachsenen $\left(\mathrm{SrTiO}_{3} / \mathrm{SrRuO}_{3}\right)_{200}$-Probe L1 (Orange) und angepassten Probe L2 (Blau). 
suchung der $\left(\mathrm{SrTiO}_{3} / \mathrm{SrTiO}_{3}\right)_{200}$ auf STO(111) Probe ist in Abbildung 5.23 in Orange zu finden. Wie auch schon im $\mathrm{LaCoO}_{3} / \mathrm{LaMnO}_{3}$-System zeigt die Gitterkonstante der L1-Probe mit $c_{L 1}=0,392 \mathrm{~nm}$ eine Verschiebung zu der Gitterkonstanten des $\mathrm{SrRuO}_{3}$ mit $c_{S R O}=0,395 \mathrm{~nm}$. Auch der Widerstand ist in Abhängigkeit von der Temperatur um ein bis zwei Größenordnungen erhöht, wie in Abbildung 5.24 in Orange zu erkennen ist. Beides deutet, ähnlich wie im Kapitel zuvor, auf ein fehlerhaftes stöchiometrisches Verhältnis der Einzellagen $(\mathrm{Ti} / \mathrm{Ru}<1)$ zueinander hin.

Darum wird eine weitere Probe L2 hergestellt, für die die Depositionsmenge des $\mathrm{SrRuO}_{3}$ um 20\% ( $V_{S R O}=7,3 \mu \mathrm{l} /$ u.c. $)$ reduziert wird. Die Ergebnisse der Röntgen- und Widerstandsmessungen sind in den Abbildungen 5.24 und 5.23 in Blau eingezeichnet. Die Gitterkonstante entspricht nun der SoA-Probe mit $c_{L 2}=0,3918 \mathrm{~nm}$, jedoch wird kein $(1 / 21 / 21 / 2)-$ Reflex beobachtet. Der Widerstand entspricht bei hohen Temperaturen der SoA-Probe, nimmt jedoch bei abnehmender Temperatur bis $90 \mathrm{~K}$ um eine Größenordnung zu. Aus zeitlichen Gründen konnten vor Abschluss der vorliegenden Arbeit keine TEM-Untersuchungen an der Probe durchgeführt werden.

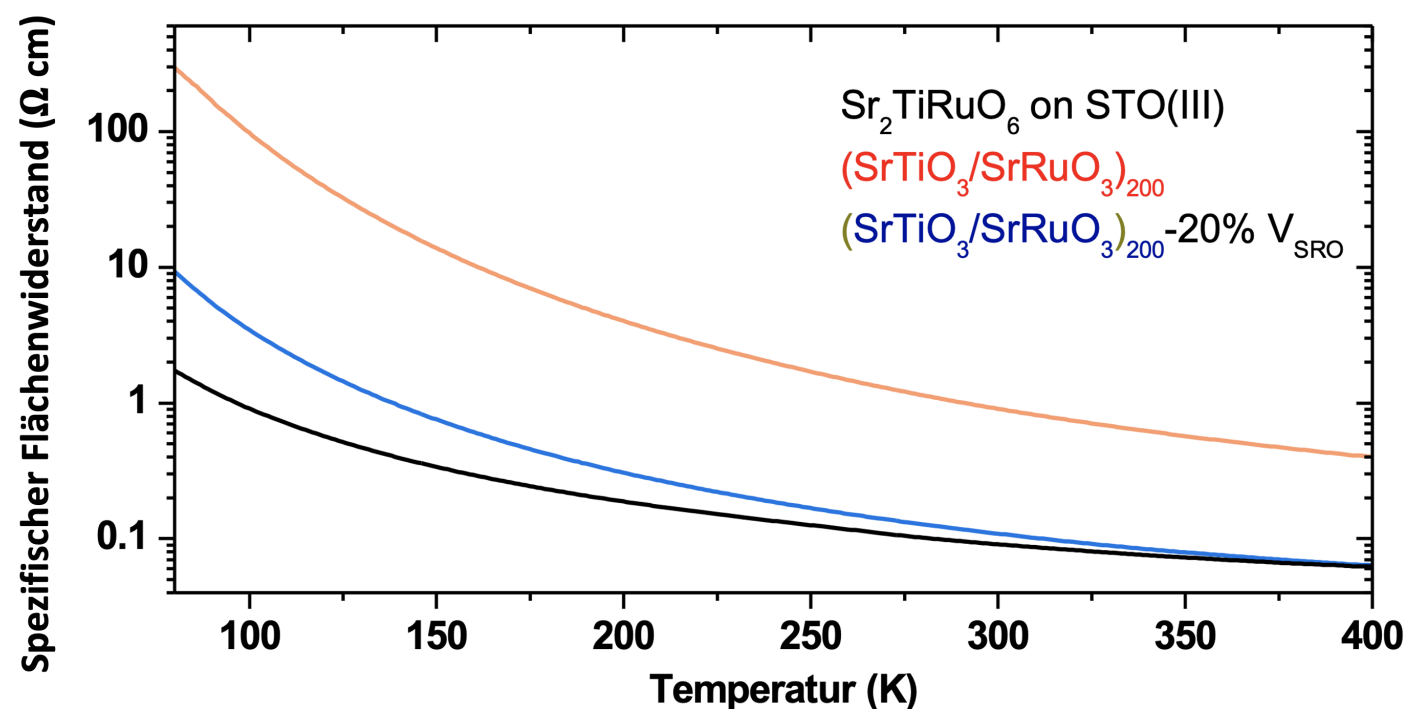

Abbildung 5.24.: Widerstandsmessung der $\mathrm{Sr}_{2} \mathrm{TiRuO}_{6}$-Referenzprobe (Schwarz) und der lagenweise aufgewachsenen $\mathrm{SrTiO}_{3} / \mathrm{SrRuO}_{3}$-Heterostrukturen L1 und L2 (Blau und Orange). 
Zusammenfassung und Diskussion Im Vergleich zu den bereits vorgestellten La-basierenden Systemen $\mathrm{La}_{2} \mathrm{NiMnO}_{6}$ und $\mathrm{La}_{2} \mathrm{CoMnO}_{6}$ führt die Valenz des $\mathrm{Sr}^{2+}$ zu einer durchschnittlichen Valenz von $4+$, die entweder über $\mathrm{Ti}^{4+} / \mathrm{Ru}^{4+}$ oder $\mathrm{Ti}^{3+} / \mathrm{Ru}^{5+}$ realisiert werden kann. Betrachtet man die jeweiligen Kationenradien, so erhöht sich ähnlich wie für $\mathrm{Ni} / \mathrm{Mn}$ und $\mathrm{Co} / \mathrm{Mn}$ der Unterschied im Kationradius, wenn die Kationen unterschiedliche Valenzen aufzeigen: $\left|R_{T i 4+}-R_{R u 4+}\right|=1,5 \mathrm{pm}<10,5 \mathrm{pm}=\left|R_{T i 3+}-R_{R u 5+}\right|$ [64]. Entsprechend liegt auch hier die Vermutung nahe, dass sich die Unterschiede in der Valenz und im Kationradius gegenseitig beeinflussen und die BPlatzordnung so weiter stabilisieren.

In der Tat wird für die SoA-Probe ein hoher Grad an B-Platzordnung erreicht, wie die TEM- und Röntgenmessungen offenbaren. Jedoch bildet sich die Ordnung innerhalb ausgedehnter Domänen aus, die in Abbildung 5.21 b) eine Größe von $\tau=6 \mathrm{~nm}$ erreichen. Diese sind durch Bereiche mit geringer B-Platzordnung voneinander getrennt. Der Ordnungsgrad der Probe lässt sich anhand der Abbildung auf ca. 60\% abschätzen und deckt sich damit sehr gut mit dem Ergebnis, das sich aus der Betrachtung des Überstrukturpeaks ergibt, $S_{\text {exp }} / S_{\text {Theo }} \approx 0,5$ [171]. Die Ausdehnung der Bereiche mit geringer B-Platzordnung verläuft zwischen den Domänen linienförmig und besitzt eine geringe Breite von $\tau_{A P B}<1 \mathrm{~nm}$, was darauf hindeutet, dass es sich um Antiphasengrenzen handelt. Diese APB in Abbildung $5.21 \mathrm{~b}$ ) verlaufen senkrecht zwischen den Domänen, womit eine Auslöschung des Überstrukturpeaks aufgrund der $\mathrm{APB}^{9}$ nicht erzeugt wird und die hohe Übereinstimmung zwischen der TEM- und XRD-Beobachtung bezüglich des Ordnungsgrades erklärt. Darüber hinaus deuten die EELS-Spektren des Titans auf das Vorhandensein von sowohl $\mathrm{Ti}^{4+}$ als auch $\mathrm{Ti}^{3+}$. Ob die jeweilige Valenz tatsächlich innerhalb der $\mathrm{SrTiO}_{3}$ - bzw. $\mathrm{SrRuO}_{3}$-Ebene vorliegt scheint allerdings fraglich zu sein. Vielmehr wird vermutet, dass das Spektrum des $\mathrm{Ti}^{4+}$ in dem Bereich geringer Ordnung gemessen wird, während das Spektrum des $\mathrm{Ti}^{3+}$ innerhalb einer Domäne aufgenommen wird. Die Messung der $\mathrm{Ti}^{3+}$ deutet auf einen Ladungstransfer zwischen dem Ruthenium und Titan hin, $\mathrm{Ti}^{4+}+\mathrm{Ru}^{4+} \rightarrow \mathrm{Ti}^{3+}+\mathrm{Ru}^{5+}$, der sich voraussichtlich innerhalb der Domänen bevorzugt ausbildet.

\footnotetext{
${ }^{9}$ Für eine Auslöschung müssten diese horizontal verlaufen.
} 
Diese Beobachtungen ermöglichen einen tiefen Einblick in das Wachstum der Probe innerhalb der MAD, die aus einer Lösung entsteht. Im Rahmen meiner Masterarbeit [168] wurde das MAD-Wachstum aus einer Lösung modelliert, was in Abbildung $5.25 \mathrm{zu}$ sehen ist. Die Aerosoltropfen nähern sich mit einer Geschwindigkeit $v_{T}$ dem Substrat mit Substrattemperatur $T_{S}$. In eionem Bereich von ca. $\leq 1 \mathrm{~mm}$ oberhalb des Substrates herrscht ein starker Temperaturgradient vor, weshalb die Tropfen entweder zerplatzen (a) oder der organische Bestandteil verdampft (b). Unmittelbar über dem Substrat entsteht eine Flüssig/Gas-Phase der Kationen mit einer Kationenkonzentration von $\zeta_{B / B^{\prime}}$. Diese Konzentration kann über die Tropfengeschwindigkeit $v_{T}$ und die Präkursorkonzentration $c_{P}$ kontrolliert werden.

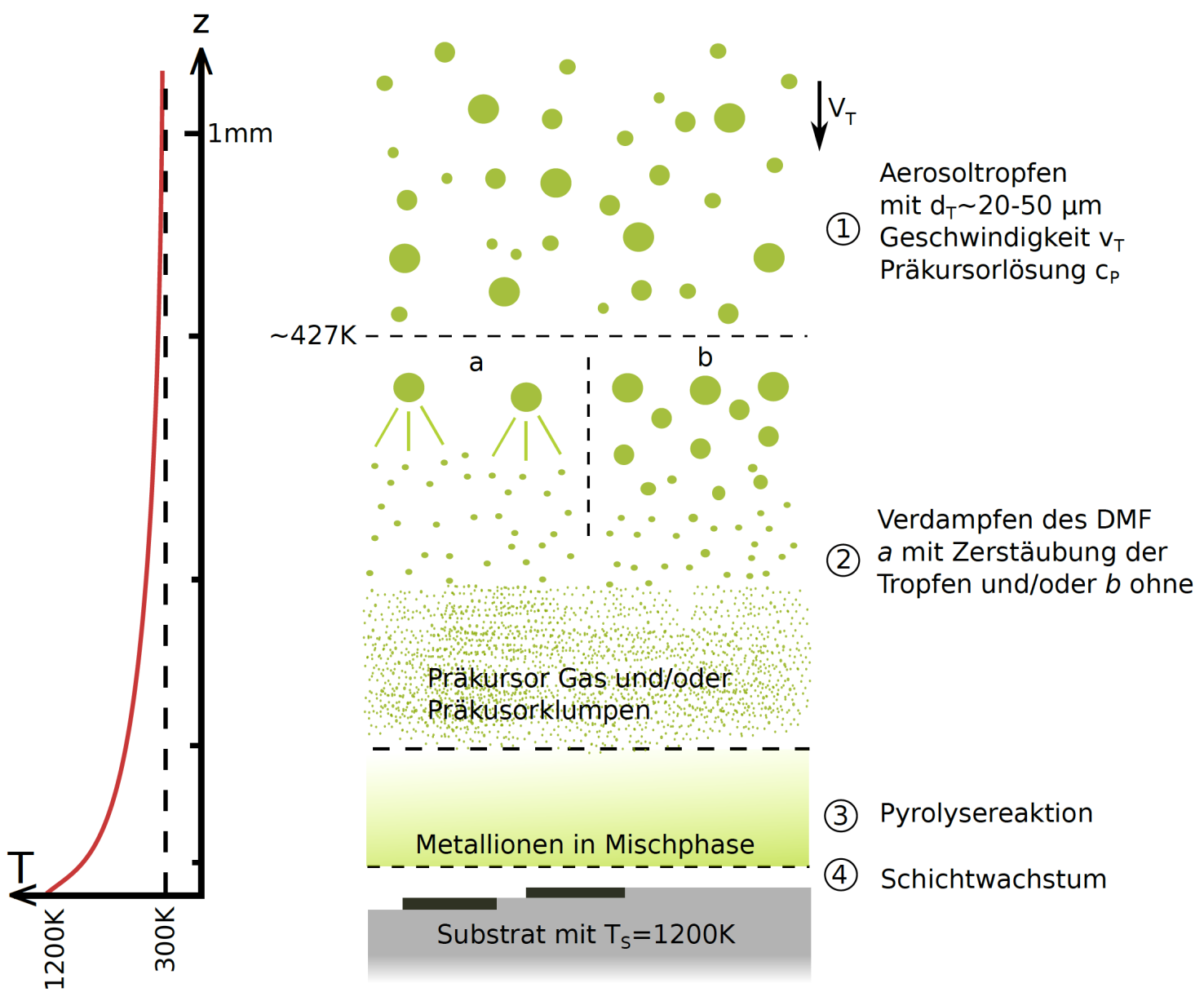

Abbildung 5.25.: Schematische Darstellung der Modellierung des Schichtwachstums mittels MAD. Mit eigener Erlaubnis aus [168]. 
Der Ursprung der B-Platzordnung ist der Ladungstransfer zwischen den beteiligten B-Kationen. Ob und inwiefern der Ladungstransfer stattfindet, hängt dabei von der Wahl der Kationen sowie von der Konzentration der hochenergetischen Flüssig/Gas-Phase ab. Hohe Wachstumstemperaturen $T_{S}$ und eine hohe Kationenkonzentration $\zeta_{B / B^{\prime}}$ führen zu einer zunehmenden Wechselwirkung zwischen den Kationen, die ab einer gewissen Energie spontan das Elektron des Ladungstransfers miteinander austauschen. Je stärker die Elektronen an das jeweilige Kation gebunden sind, desto höher muss die Kationenkonzentration $\zeta_{B / B^{\prime}}$ und die einhergehende Energie sein, um den Ladungstransfer auszulösen. Der Abstand der freien Kationen in der Flüssig/Gas-Phase oberhalb des Substrates $\lambda_{k r i t}$ muss dabei schätzungsweise unterhalb von $\lambda_{k r i t} \leq 1 \mathrm{~nm}$ liegen, sodass die Kationenkonzentration $\zeta_{B / B^{\prime}} \approx 10^{21} \mathrm{~cm}^{-3}$ beträgt. Der Ladungstransfer führt zu einem erhöhten Unterschied der Kationradien $\Delta Z$, der das Ordnungsphänomen weiter verstärkt, womit die bislang freien Kationen in der Umgebung zur Ordnung und zu einem weiteren Ladungstransfer gezwungen werden.

Es bilden sich Keimzentren, aus denen die B-Platzordnung selbstverstärkend entsteht, die schließlich zu den beobachteten Domänen führt. Für $\mathrm{Sr}_{2} \mathrm{TiRuO}_{6}$ sind diese Kräfte jedoch nicht stark genug, die Ordnung über die gesamte Probe zu stabilisieren. Oder aber die treibenden Kräfte gleichen sich gegenseitig aus und neutralisieren sich, wenn zwei benachbarte Domänen beim Wachstum aufeinandertreffen. Somit bilden sich Anitphasengrenzen und vergleichsweise kleine Bereiche, in denen sich keine B-Platzordnung ausgebildet hat und mit Ti- und Ru-Kationen in ihrer $4+$ Valenz.

Das Wachstum über die abwechselnde Deposition der Monolagen unterscheidet sich grundlegend vom soeben beschriebenem Wachstum aus einer Lösung. Mit jeder Lage bildet sich ein geschlossener Film mit seiner jeweiligen Valenzverteilung. Ob nach der Deposition einer weiteren Lage der Ladungstransfer stattfindet, hängt von dem jeweiligen System ab und wie sehr es die Elektronen an sich bindet. Während für $\mathrm{LaMnO}_{3}$ und $\mathrm{La}(\mathrm{Ni}, \mathrm{Co}) \mathrm{O}_{3}$ ein Ladungstransfer beobachtet wird, ist dies für $\mathrm{SrTiO}_{3}$ nicht automatisch gegeben, da die Titan $4+$ Valenz in sich sehr stabil ist. 
Die lagenweise Deposition der Einzelschichtsysteme zur künstlichen Erzeugung der B-Platzordnung kann dabei helfen, die Ausbildung der beobachteten APB durch das Wachstum geschlossener Einzelschichten zu verhindern. Wie im Kapitel davor dargestellt, wird jedoch zu Beginn für die L1-Probe nicht das passende stöchiometrische Verhältnis erzielt. Aufbauend auf den Erkenntnissen aus dem $\mathrm{LaCoO}_{3} / \mathrm{LaMnO}_{3}$-System konnte eine Probe L2 hergestellt werden, die vergleichbare Eigenschaften wie SoA-Probe aufweist. Diese Ähnlichkeit deutet auf einen mindesten genau so hohen Ordnungsgrad von bis zu 60\% innerhalb der L2-Probe hin, wie in der SoA-Probe. Die Wachstumsbedingungen sind jedoch grundlegend anders. Um zu verstehen, wie das LbL-Wachstum das System beeinflusst, sind TEM-Untersuchungen unerlässlich. Verglichen mit der SoA-Probe, wächst die LbL-Probe nicht aus einer Lösung, wodurch der selbstverstärkende Effekt der treibenden Kräfte zur Ausbildung der B-Platzordnung weitestgehend unterdrückt sein müsste und die Ausbildung der Domänen verhindert werden sollte.

Wie bereits erwähnt, ist das Titan in seiner $4+$ Valenz eine sehr stabile Konfiguration. Dadurch ist es durchaus vorstellbar, dass sich die B-Platzordnung im LbL-Modus ohne Ladungstransfer einstellt, weil die abgeschlossenen Einzelschichten $\mathrm{SrTiO}_{3}$ und $\mathrm{SrRuO}_{3}$ in sich stabil sind und in ihrer $\mathrm{Ti}^{4+}$ - bzw. $\mathrm{Ru}^{4+}-$ Valenz bleiben. Dies wäre eine ganz neue Beobachtung im Zusammenhang mit der B-Platzordnung und würde das Verständnis des Wachstums weiter vertiefen. Es würde zeigen, dass für stabile Systeme das Wachstum aus einer Lösung heraus notwendig ist, um den Ladungstransfer hervorzurufen. Um diese Fragen eindeutig zu klären, sind auch hier TEMUntersuchungen dringend notwendig, die leider aus zeitlichen Gründen im Rahmen der vorliegenden Arbeit nicht mehr zu realisieren sind.

Trotz der offenen Fragen bestätigen die vorgestellten Ergebnisse der L1und L2-Probe, dass sich das Verfahren des stöchiometrischen Lagenwachstums gut eignet, um zuverlässig Doppelperowskite durch abwechselnde Deposition der $\mathrm{ABO}_{3}$ - und $\mathrm{AB}^{\prime} \mathrm{O}_{3}$-Einzelschichtsysteme in (111)-Orientierung herzustellen. Daraus stellt sich abschließend die Frage, inwiefern sich die vorgestellte Methodik eignet, um auf andere Materialsysteme bis hin zu anderen Wachstumsmethoden übertragen zu werden. 
Die erfolgreiche Abscheidung der Heterostruktur $\mathrm{LaCoO}_{3} / \mathrm{LaMnO}_{3}$ zeigt, dass die B-Platzordnung durch das LbL-Wachstum nach Optimierung im Vergleich zu der SoA $\mathrm{La}_{2} \mathrm{CoMnO}_{6}$ weiter erhöht werden kann. Voraussetzung dafür ist eine hohe Schichtqualität der Einzelschichtsysteme, die mit derselben Wachstumsmethode (in dem Fall MAD) gewachsen werden. Dementsprechend kann davon ausgegangen werden, dass das lagenweise Wachstum der DP zur Herstellung künstlicher B-Platzordnung auch auf die üblichen und etablierten Wachstumsverfahren (PLD, MBE, Sputtern usw.) übertragen werden kann, wenn die Einzelschichtsysteme zuverlässig hergestellt werden können.

Diese Vorgehensweise muss natürlich für jedes Materialsystem im Zusammenhang mit der jeweiligen Wachstumsmethode neu bewertet werden. Die MBE könnte beispielsweise verwendet werden, um die Kaltionordnung in nicht-oxidischen Materialien zu synthetisieren, die bei der MAD aufgrund des atmosphärischen Drucks ausgeschlossen sind. Da die Einzelschichtsysteme $\mathrm{LaMO}_{3}$ mit $\mathrm{M}=\mathrm{Co}, \mathrm{Mn}$ und Ni auch mittels PLD oder Sputtern [37,49, 157-164, 167] zuverlässig hergestellt werden können, sollte das vorgestellte Prinzip auch innerhalb dieser Wachstumsmethoden angewandt werden können. Andererseits zeigt das Beispiel der entstehenden DP-Phase sowohl bei $\mathrm{LaCoO}_{3} / \mathrm{LaMnO}_{3}$ - als auch $\mathrm{LaNiO}_{3} / \mathrm{LaMnO}_{3}$-Heterostrukturen, dass sich die Wachstumskinetik grundsätzlich von der Wachstumskinetik der PLD unterscheiden muss. Im Fall von DP $\mathrm{LaCoO}_{3} / \mathrm{LaMnO}_{3}$ und $\mathrm{LaNiO}_{3} / \mathrm{LaMnO}_{3}$ scheinen die MAD-Wachstumsbedingungen das Wachstum einer geordneten Phase zu begünstigen, da diese bisher noch nicht in den jeweiligen Heterostrukturen beobachtet wird [82,83,173-175]. Aus dieser Beobachtung kann jedoch nicht geschlossen werden, dass dies für alle Materialien gelten muss. Beispielsweise wurden mit der MAD auch Versuche zur künstlichen Synthese der B-Ordnung innerhalb des Doppelperowskits $\mathrm{Sr}_{2} \mathrm{FeMoO}_{6}$ durchgeführt. Das Einschichtsystem $\mathrm{SrFeO}_{3}$ konnte jedoch aufgrund des nicht optimalen Sauerstoffpartialdruckes nicht zuverlässig mit dem MAD gewachsen werden. Daher kann die vorgestellte Methode nicht darauf angewendet werden. Wohingegen die Vakuumbedingungen innerhalb der PLD beispielsweise das Wachstum von $\mathrm{SrFeO}_{3}$ ermöglichen, was bereits für $\mathrm{SrFeO}_{3} / \mathrm{SrRuO}_{3}{ }^{-}$ Heterostrukturen angewandt wird [176]. 


\begin{abstract}
Abschließend lässt sich zusammenfassen, dass die lagenweise Deposition der Einzelschichtsysteme zu einer Verbesserung bzw. Erhöhung der Kationordnung innerhalb von Doppelperowskiten in (111)-Orientierung führen kann, sofern die Einzelschichtsysteme mit der jeweiligen Wachstumsmethode zuverlässig wachsen und geschlossene Filme bilden.

Diese Erkenntnis bildet eine neue Technik für das Wachstum von kationgeordneten korrelierten Oxidschichten und kann einen wesentlichen Durchbruch sowohl für die Grundlagenforschung als auch für die angewandte Forschung liefern. Potenzielle Anwendungen würden stark von der Entwicklung dieser fortschrittlichen Wachstumsmethode profitieren, die kontrollierbar und reproduzierbar einen hohen Grad an Kationordnung durch Feinabstimmung der Wachstumsbedingungen auf atomarer Skala herstellen kann.
\end{abstract}




\subsection{Grenzflächeneffekte und Kationordnung in $3 d / 5 d$-Übergittern}

Rechtliches und Lizenzen Das folgende Kapitel fasst einen weiten Teil der wissenschaftlichen Ergebnisse der vorliegenden Promotion zusammen. Zusätzlich werden die Ergebnisse der Arbeitsgruppe um Philipp Gegenwart aus der Universität Augsburg vorgestellt. Die jeweiligen Beiträge der externen Gruppe werden entsprechend gekennzeichnet. Zum Zeitpunkt der vorliegenden Dissertation sind die Ergebnisse in keiner Publikation veröffentlicht, weshalb nach bestem Wissen kein Interessenkonflikt vorliegt.

\subsubsection{Grenzflächeneffekte und Kationordnung in $\mathrm{SrMnO}_{3} / \mathrm{SrlrO}_{3}-$ Heterostrukturen auf STO(100)}

Im folgenden Kapitel werden die Erkenntnisse des Lagenwachstums zur Herstellung künstlicher B-Platzordnung ein weiteres Mal genutzt und auf die 3d/5d-Heterostruktur aus $\mathrm{SrMnO}_{3} / \mathrm{SrIrO}_{3}$ angewandt.

Motiviert durch die Suche nach dem anomalen und topologischen Halleffekt sind im vorliegenden System neben der Kationordnung die Grenzflächeneffekte von besonderem Interesse. Deshalb wird wie bereits für $\mathrm{LaNiO}_{3} /$ $\mathrm{LaMnO}_{3}$ im Kapitel 5.1.1 die Schichtdicke der Einzelschichtsysteme stetig reduziert. Es werden Übergitter aus $\left(\mathrm{SrIrO}_{3}\right)_{n} /\left(\mathrm{SrMnO}_{3}\right)_{n}$ mit $n=5,2$ und 1 hergestellt und auf die Grenzflächeneffekte hin untersucht. Da sowohl der anomale als auch der topologische Halleffekt bisher vorzugsweise in Heterostrukturen entlang der (100)-Orientierung beobachtet werden [16-18, 20,21], werden anders als bisher die vorgestellten Proben auf STO in (100)-Richtung gewachsen. Im Zuge dessen wird erprobt, ob sich die Herstellung künstlicher B-Platzordnung durch die lagenweise Deposition der Einzelschichtsysteme auch auf die (100)-Orientierung zuverlässig übertragen lässt und so eine B-Platzordnung hervorruft, die so beim Wachstum aus einer Lösung (SoA) in dem System nicht natürlich vorkommt. 
Einzelschichtsysteme $\mathrm{SrlrO}_{3}$ und $\mathrm{SrMnO}_{3} \quad$ Mit $c_{S I O, B u l k}=0,394 \mathrm{~nm}$ [17] und $c_{S M O, B u l k}=0,380 \mathrm{~nm}$ [17] weichen die pseudokubischen Gitterkonstanten der Bulkproben von $\mathrm{SrIrO}_{3}$ und $\mathrm{SrMnO}_{3}$ deutlich von der Konstanten des Substrates, $c_{S T O}=0,3905 \mathrm{~nm}$, ab, weshalb beim Wachstum beider Systeme auf STO deutliche Verspannungszustände entstehen. Dadurch kommt es bereits nach einer geringen Schichtdicke zu einer Relaxation des Systems, in dem die Schicht ins sogenannte Inselwachstum ${ }^{10}$ übergeht und mit ihren ursprünglichen Gitterparametern weiterwächst. Um diesen Effekt entgegenzuwirken, wird für die folgenden Proben die Depositionstemperatur auf $T_{D e p} \approx 700{ }^{\circ} \mathrm{C}$ reduziert. Damit wird eine geringere thermische Energie beim Wachstum erreicht, die die Relaxation des Systems verzögern soll und so das Wachstum dickerer Einzelschichtlagen ermöglicht.

Für $\mathrm{SrMnO}_{3}$ ist bekannt, dass diese Verspannungen nach wenigen Atomlagen durch Aufspaltung der Schicht zu relaxieren beginnen [124]. Dadurch werden die Einstellproben für $\mathrm{SrMnO}_{3}$ nur mit einer geringen Schichtdicke von wenigen Nanometern $(3-5 \mathrm{~nm})$ zur Einstellung des Materialsystemes hergestellt [116]. Da $\mathrm{SrMnO}_{3}$ ein antiferromagnetischer Isolator ist $\left(T_{N} \approx 233 \mathrm{~K}\right)$ [177] und für die Herstellung der Heterostrukturen eine glatte Morphologie notwendig ist, wird das System auf die Oberflächenrauigkeit hin optimiert ${ }^{11}$. Die erzielte Oberflächenmorphologie ist im AFMBild in Abbildung A.8 im Anhang zu sehen. Die Schicht weist einen RMSWert von $R_{q, S M O}=0,417 \mathrm{~nm}$ auf. Dabei wird ein molares Verhältnis von $\mathrm{Sr} / \mathrm{Mn}=1,36$ für die Lösung verwendet. Für die Depositionsmenge einer Lage mit $d_{S M O}=1$ u.c. ergibt sich ein Wert von $V_{S M O}=11,5 \mu l /$ u.c..

Während $\mathrm{SrMnO}_{3}$ auf dem Substrat unter Zugspannung aufwächst, ergibt sich aufgrund der vergleichbar hohen Gitterkonstanten für $\mathrm{SrIrO}_{3}$ der entgegengesetzte Trend. Abbildung 5.26 a) zeigt die Ellipsometrie-Messung einer $\mathrm{SrIrO}_{3}$-Probe auf $\mathrm{STO}(100)$ mit der Schichtdicke von $d_{S I O}=4,5 \mathrm{~nm}$, für die $150 \mu l$ der Präkursorlösung deponiert werden. Es sind drei Bereiche in-

\footnotetext{
${ }^{10} 3 \mathrm{D}-$ Wachstum, bei dem die kommenden Monolagen bereits zu wachsen beginnen, bevor die vorherigen einen geschlossenen Film bilden.

${ }^{11}$ Kann in mehreren Abschlussarbeiten genau nachvollzogen werden [124,169, 179].
} 

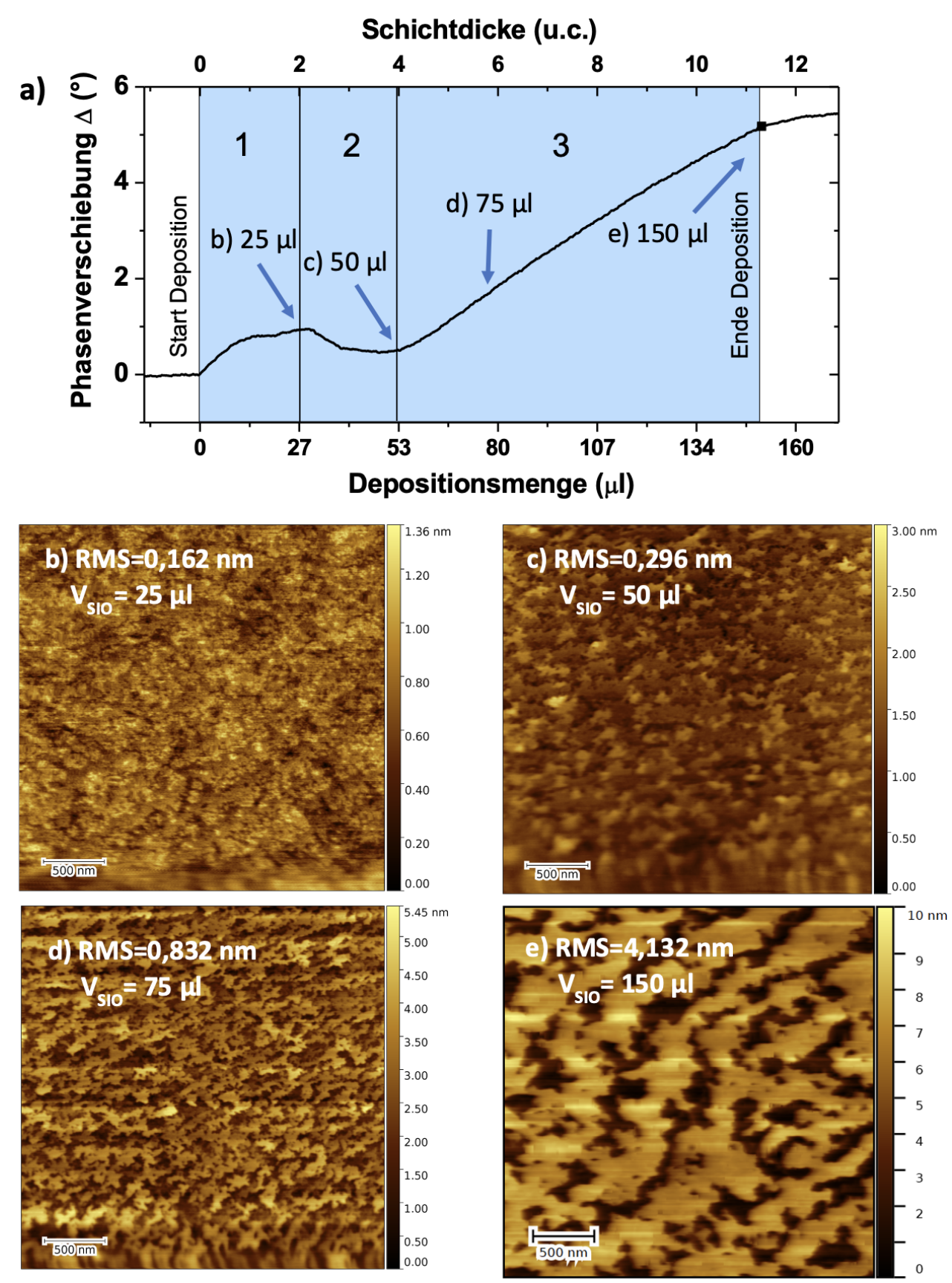

Abbildung 5.26.: a) Ellipsometrieparameter $\Delta$ der $\mathrm{SrIrO}_{3}$-Probe auf STO(100). Es sind drei Bereiche innerhalb des Wachstumes zu erkennen, die auf unterschiedliche Wachstumsmoden in Verbindung mit dem Verspannungszustand zurückzuführen sind. b) - e) AFM-Aufnahmen von $\mathrm{SrIrO}_{3^{-}}$ Proben auf (100) bei einer Depositionsmenge von $25 \mu \mathrm{l}$, $50 \mu l, 75 \mu l$ und $150 \mu l$. 
nerhalb der Messung erkennbar. Zunächst steigt das Signal innerhalb der ersten zwei Einheitszellen an (Bereich 1), bevor es für die darauffolgenden zwei Lagen wieder sinkt (Bereich 2). Erst danach scheint sich das Wachstum zu stabilisieren und es stellt sich eine konstante Steigung ein. In Verbindung mit den Simulationen zu der Messung ergibt sich aus der Steigung im 3. Bereich ein komplexer Brechungsindex von $N_{S I O}=2.344-0.421 i$. Abbildung 5.26 e) zeigt die Oberflächenmorphologie der Probe. Es ist deutlich zu erkennen, dass kein geschlossener Film entsteht, was die Folge der Relaxation des Verspannungszustandes auf dem STO-Substrat ist.

Um festzustellen, ab welcher Schichtdicke das System anfängt zu relaxieren und wie sich dieses Verhalten in der Ellipsometriemessung widerspiegelt, werden weitere Proben mit unterschiedlichen Schichtdicken hergestellt, in dem $25 \mu l, 50 \mu l$ und $75 \mu l$ der Lösung deponiert werden. Die resultierenden AFM-Bilder sind in Abbildung 5.26 b) - d) zu finden. Man erkennt, dass für geringe Depositionsmengen $V_{D e p}<50 \mu l$ ein geschlossener Film aufwächst mit einer Oberflächenrauigkeit von $R_{q}<0,3 \mathrm{~nm}$. Erhöht man darüber hinaus die Schichtdicke $d_{S I O}>4$ u.c. (Bereich 3), erhöht sich die Oberflächenrauigkeit deutlich, $R_{q}=0,832$, wie in Abbildung $5.26 \mathrm{~d}$ ) zu erkennen ist. Der Wachstumsmodus entspricht dem Stranski-Krastanov-Wachstum, nach dem die ersten Lagen geschlossen aufwachsen, bevor das Inselwachstum stattfindet $[180,181]$. Es ist davon auszugehen, dass bereits ab einer Schichtdicke von 4 u.c. kein geschlossener Film vorliegt, der für das Wachstum von zuverlässigen Heterostrukturen notwendig ist. Aus diesem Grund werden die folgenden Proben nur bis zu einer Einzelschichtdicke von 5 u.c. hergestellt. Das liegt damit knapp über dem Limit der Einzelschichtsysteme liegt. Für $\mathrm{SrIrO}_{3}$ ergibt sich aus den vorgestellten Proben eine Depositionsmenge von $V_{S I O}=12,7 \mu l /$ u.c. für eine Einheitszelle in (100)-Orientierung. Das Verhältnis Sr/Ir in der Lösung beträgt 0,95.

\section{Strukturelle Eigenschaften der Heterostrukturen in (100)-Orientierung}

Ausgehend von den Wachstumsbedingungen der Einzelschichtsysteme werden die Heterostrukturen $\left(\mathrm{SrIrO}_{3}\right)_{n} /\left(\mathrm{SrMnO}_{3}\right)_{n}$ mit $n=5,2$ und 1 auf $\mathrm{STO}(100)(\mathrm{SL}(\mathrm{n} / \mathrm{n}))$ gewachsen, beginnend mit dem SL(5/5). Die Deposition startet mit $\mathrm{SrMnO}_{3}$ und schließt mit $\mathrm{SrIrO}_{3}$ ab. Die Anzahl an Wie- 

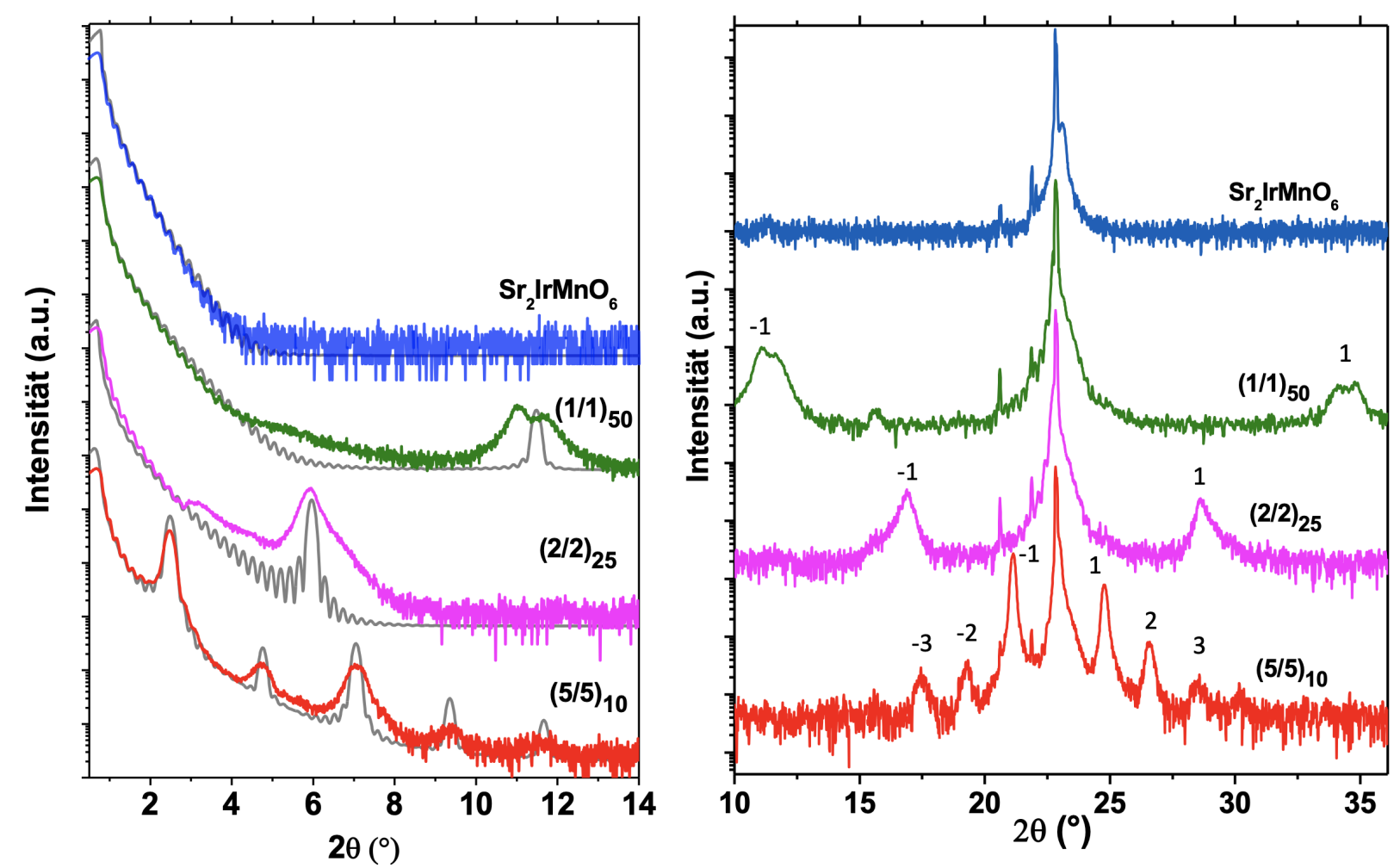

Abbildung 5.27.: Röntgenuntersuchungen an den $\left(\mathrm{SrIrO}_{3}\right)_{n} /\left(\mathrm{SrMnO}_{3}\right)_{n^{-}}$ Proben mit $n=5,2$ und 1. a) XRR-Messungen mit den jeweiligen numerischen Anpassungen, die mit GenX durchgeführt werden (Grau), b) XRD-Messungen.

derholungen ist so gewählt, dass die Probe aus 100 Einheitszellen besteht, was einer Schicht von $d=37,8 \mathrm{~nm}$ entspricht.

Abbildung 5.27 a) zeigt die XRR-Messungen der Proben und die dazugehörigen numerische Anpassung gemäß dem Parratt-Formalismus (in Grau). Die AFM-Aufnahmen der Oberflächenmorphologie sind in Abbildung A.9 im Anhang zu finden. Die Ergebnisse der Simulation und die RMS-Werte sind in Tabelle 5.3 zusammengefasst. Für die (5/5) Probe wird mit einer Abweichung von 1\% die theoretische Gesamtschichtdicke erreicht. Die Schichtdicken der Einzelschichtsysteme entsprechen nach der numerischen Anpassung sehr gut den zu erwartenden Werten. Die Oberflächenmorphologie zeigt jedoch eine vergleichsweise raue Struktur mit einem hohen RMS-Wert von $R_{q,(5 / 5)}=1,15 \mathrm{~nm}$, was auf die bereits erwähnten Verspannungszustände des $\mathrm{SrIrO}_{3}$ und $\mathrm{SrMnO}_{3}$ zurückzuführen ist. Sowohl die XRR- als auch die XRD-Messungen zeigen ausgeprägte Peaks der Überstruktur, was auf 
scharfe Grenzflächen deuten lässt. TEM-Aufnahmen der (5/5) Probe sind in Abbildung 5.28 a) und b) gezeigt. Die einzelnen Monolagen sind in Abbildung $5.28 \mathrm{~b})$ in Orange $\left(\mathrm{SrMnO}_{3}\right)$ und Blau $\left(\mathrm{SrIrO}_{3}\right)$ hervorgehoben. Es ist $\mathrm{zu}$ erkennen, dass für die jeweiligen Einzellagen die Schichtdicke von fünf Einheitszellen nicht durchgängig erreicht wird. Dabei fallen die $\mathrm{SrMnO}_{3}$ Schichten mit 4 u.c. geringer aus, während die $\mathrm{SrIrO}_{3}$-Lagen eine Einzellagendicke von bis zu 6 u.c. erreichen, was sich nicht mit den Ergebnissen der numerischen Anpassung der Röntgenuntersuchung deckt.

Wird die Einzelschichtdicke auf $n=2$ reduziert, erhöht sich die Abweichung zum theoretischen Wert der Schichtdicke auf 2,4\%, wohingegen die Oberflächenrauigkeit drastisch reduziert ist. Mit $R_{q,(2 / 2)}=0,37 \mathrm{~nm}$ befindet sich die Rauigkeit in der Größenordnung einer Atomlage. Die geringe Einzelschichtdicke führt dementsprechend nicht zum 3D-Inselwachstum und bildet abschließende Lagen. Wie für die $(5 / 5)$ Probe werden in der Weit- und Kleinwinkelmessung die Peaks der Überstruktur beobachtet. Der Peak der XRR-Messung besitzt jedoch eine hohe Halbwertsbreite, die auf eine Abweichung der idealen Überstruktur hinweist. Die TEM-Aufnahmen dieser Probe in Abbildung 5.28 c) und d) bestätigen diese Beobachtung. In Abbildung $5.28 \mathrm{~d}$ ) sind durch die blauen und orangene Linien die einzelnen Monolagen hervorgehoben, wodurch exemplarisch sichtbar wird, dass für einzelne Lagen eine Abweichung von $n=2$ vorliegt.

\begin{tabular}{c|ccccc}
\hline \hline & $\begin{array}{c}\text { Schicht- } \\
\text { dicke } \\
d[\mathrm{~nm}]\end{array}$ & $\Delta d$ & $\begin{array}{c}\text { Einzellagen- } \\
\text { dicke } \mathrm{SrIrO}_{3} \\
d_{S I O}[\mathrm{~nm}]\end{array}$ & $\begin{array}{c}\text { Einzellagen- } \\
\text { dicke } \mathrm{SrMnO}_{3} \\
d_{S M O}[\mathrm{~nm}]\end{array}$ & RMS \\
{$\left[(5 / 5)_{10}\right.$} & 38,3 & 1,0 & 1,93 & 1,9 & 1,51 \\
$(2 / 2)_{25}$ & 37,78 & 2,4 & 0,75 & 0,76 & 0,37 \\
$(1 / 1)_{50}$ & 38,85 & $-0,4$ & 0,39 & 0,38 & 0,29 \\
\hline $\mathrm{SoA}$ & 37,2 & - & - & - & 0,332 \\
$\mathrm{SrIrO}_{3}$ & 0,8 & - & - & - & 0,162 \\
$\mathrm{SrMnO}_{3}$ & 5,1 & - & - & - & 0,417 \\
\hline \hline
\end{tabular}

Tabelle 5.3.: Ergebnisse der strukturellen Untersuchung mittels XRRMessung in Verbindung mit der numerischen Anpassung und der AFM-Aufnahmen der Übergitter $(n / n)$, der SoA $\mathrm{Sr}_{2} \mathrm{IrMnO}_{6}$-Probe und der Einzelschichtsysteme $\mathrm{SrIrO}_{3}$ und $\mathrm{SrMnO}_{3}$ auf $\mathrm{STO}(100)$. 


\section{SL $(5 / 5)$}
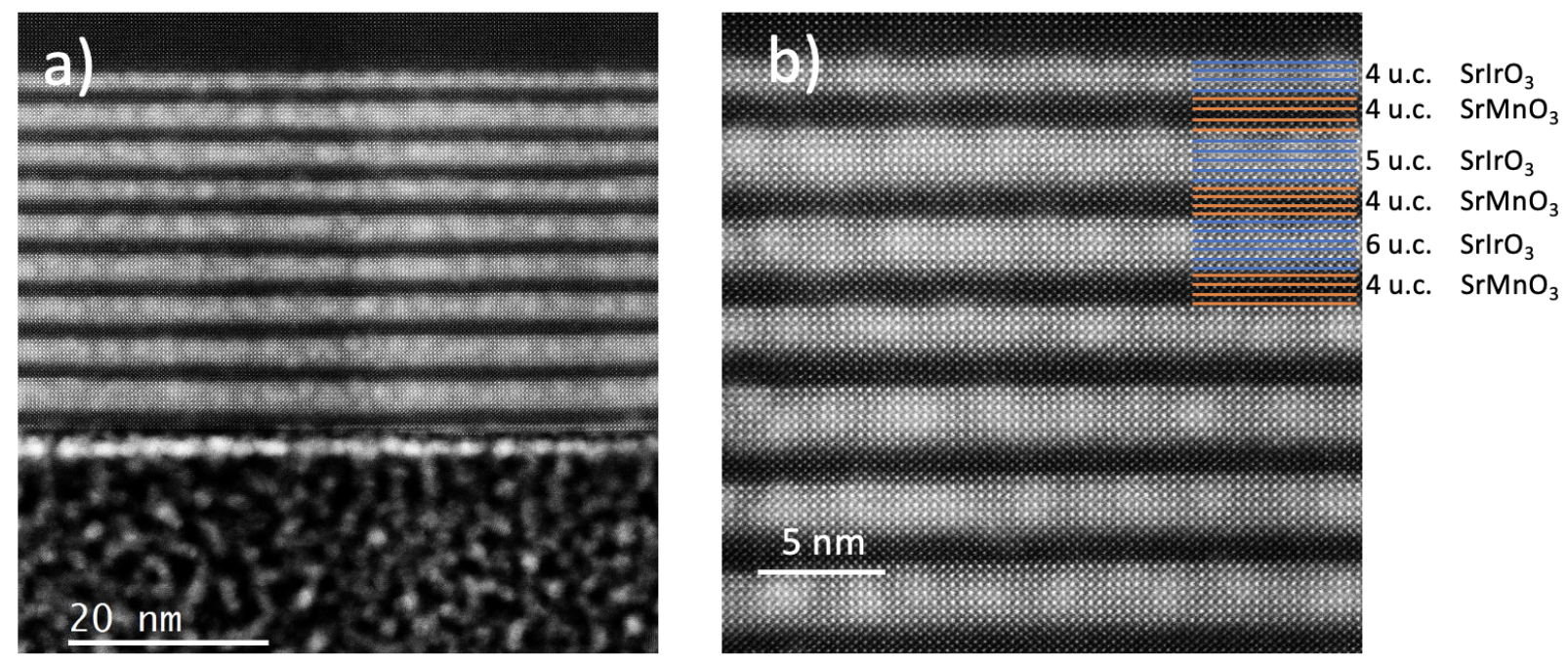

\section{$\operatorname{SL}(2 / 2)$}
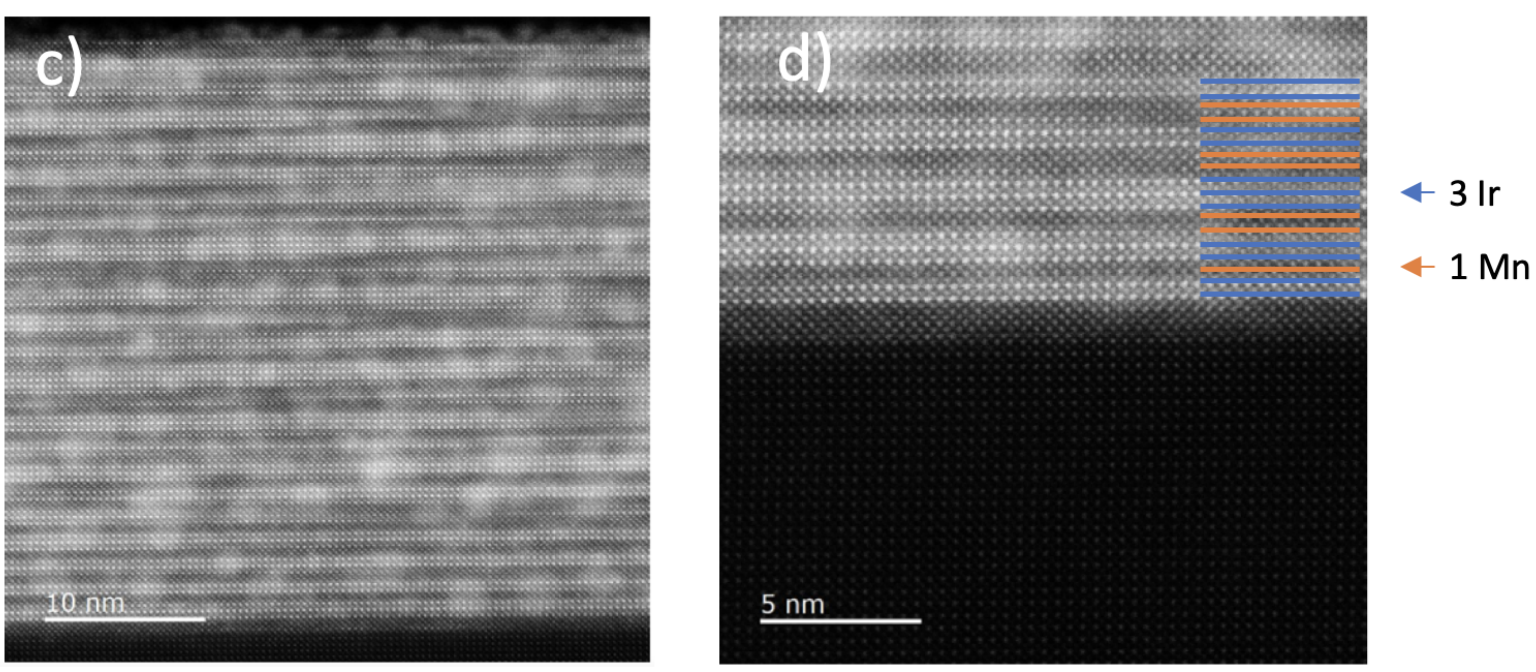

Abbildung 5.28.: HAADF STEM-Aufnahme der a,b) $\left(\left(\mathrm{SrIrO}_{3}\right)_{5} /\right.$ $\left.\left(\mathrm{SrMnO}_{3}\right)_{5}\right)_{10}$ und der c,d $) \quad\left(\left(\mathrm{SrIrO}_{3}\right)_{2} /\left(\mathrm{SrMnO}_{3}\right)_{2}\right)_{25^{-}}$ Probe. a,c) Gesamtaufnahme und b,d) Vergrößerung. Zur Bestimmung der jeweiligen Einzelschichtdicken sind in Blau $\left(\mathrm{SrIrO}_{3}\right)$ und Orange $\left(\mathrm{SrMnO}_{3}\right)$ die Einheitszellen einer Lage hervorgehoben. Die Aufnahmen sind von Robert Gruhl am Lehrstuhl für Experimentalphysik VI an der Universität Augsburg angefertigt worden. 


\section{SL (1/1)}

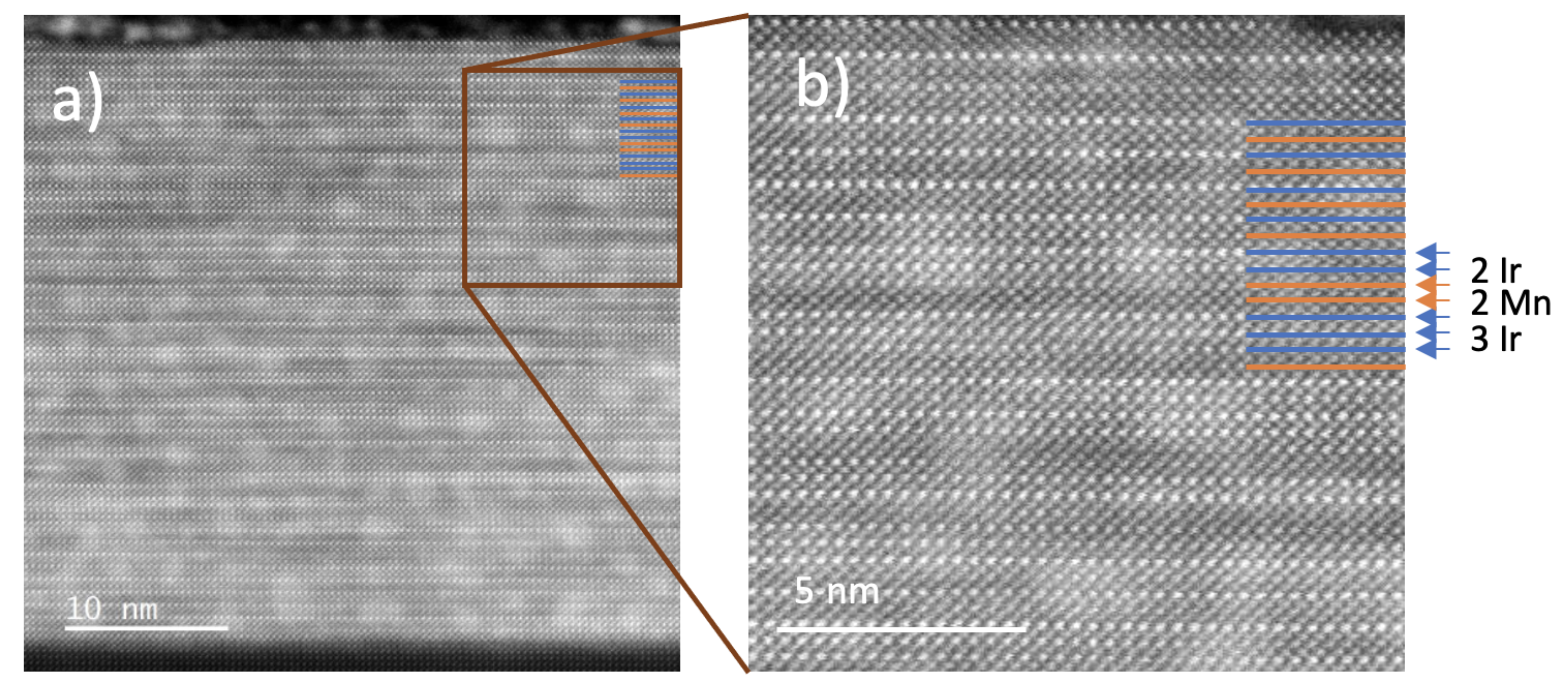

Abbildung 5.29.: HAADF STEM-Aufnahme der $\left(\left(\mathrm{SrIrO}_{3}\right)_{1} /\left(\mathrm{SrMnO}_{3}\right)_{1}\right)_{50^{-}}$ Probe. a) Gesamtaufnahme und b) Vergrößerung. Zur Bestimmung der jeweiligen Einzelschichtdicken sind in Blau $\left(\mathrm{SrIrO}_{3}\right)$ und Orange $\left(\mathrm{SrMnO}_{3}\right)$ in b) die Einheitszellen einer Lage hervorgehoben. Die Aufnahme ist von Robert Gruhl am Lehrstuhl für Experimentalphysik VI an der Universität Augsburg angefertigt worden

Mit einer Abweichung von $\Delta d=-0,4 \%$ wird für das $\operatorname{SL}(1 / 1)$ (LbLProbe) die erwartete Gesamtschichtdicke sehr genau erreicht. Mit $R_{q,(1 / 1)}=$ 0,29 $\mathrm{nm}$ weist die LbL-Probe die glatteste Oberfläche auf, dessen RMS-Wert unter dem der SoA-Probe mit $R_{q, S o A}=0,33 \mathrm{~nm}$ (siehe Abbildung A.9 im Anhang) liegt. Wie für die Proben zuvor wird der Peak der Überstruktur beobachtet, der jedoch aus einem Doppelpeak besteht. Dies weist erneut auf eine fehlerhafte Periodizität innerhalb der Heterostruktur hin, was durch die TEM-Aufnahmen in Abbildung 5.29 auch hier bestätigt wird. Einzelne Monolagen bestehen jeweils aus zwei bis drei Einheitszellen.

Kationordnung der LbL-Probe Bevor die Grenzflächeneigenschaften weiter vertieft werden, soll die Kationordnung mit der resultierenden Ordnungsform betrachtet werden und mit der SoA-Referenzprobe verglichen werden. Zwar zeigen die Abweichungen innerhalb der Periodizität, dass die lagenweise Ordnung nicht vollständig erzeugt wird, dennoch deutet der Über- 
strukturpeak der Röntgenuntersuchung darauf, dass die Ordnung über weite Teile der Probe ausgebildet sein muss. Die Mitte des Doppelpeaks der Überstruktur befindet sich bei $2 \Theta=11,4^{\circ}$, woraus sich für die Schichtdicke einer Doppellage der Wert $d_{S I O+S M O}=0,776 \mathrm{~nm}$ bestimmen lässt. Der Wert passt somit sehr genau zu der Dicke der erwarteten Doppellage, $d_{S I O+S M O} \approx c_{S I O}+c_{S M O}=0,394 \mathrm{~nm}+0,38 \mathrm{~nm}$.

Um zu untersuchen, ob und inwiefern sich die B-Platzordnung im SoASystem natürlich einstellt, wird neben der SoA-Referenzprobe auf STO(100) eine weitere Probe in (111)-Orientierung hergestellt. Die XRD-Messung ist in Abbildung A.10 im Anhang zu finden. Die SoA-Probe in (111)-Orientierung weist bei $2 \Theta=19,8^{\circ}$ einen Überstrukturpeak auf, der auf das Vorhandensein von B-Platzordnung schließen lässt. Wie die bereits vorgestellten Materialsysteme ordnet sich somit auch das $\mathrm{Sr}_{2} \mathrm{IrMnO}_{6}$ in der $\mathrm{NaCl}$ Struktur ${ }^{12}$. Die künstlich erzeugte B-Platzordnung entlang der (100)-Orientierung entspricht hingegen der Lagenordnung [6,182], die natürlich nur in wenigen Doppelperowskiten vorzufinden ist. Die lagenweise Deposition der Einzelschichtsysteme eignet sich somit auch, die B-Platzordnung entlang anderer Orientierungen herzustellen, die natürlich nicht vorkommen. Die lagenweise Ordnung hat großen Einfluss auf die Eigenschaften des Materialsystemes, wie die folgenden Untersuchungen zeigen.

Magnetische und elektrische Eigenschaften Um die Grenzflächeneffekte und den Einfluss der Einzelschichtdicke auf das System zu untersuchen, werden Magnetisierungsmessungen an den Proben durchgeführt, die in Abbildung $5.30 \mathrm{zu}$ sehen ist. Für die $\mathrm{SL}(\mathrm{n} / \mathrm{n})$ mit $\mathrm{n}=5,2,1$ und der SoA-Probe werden die Messungen feld- und temperaturabhängig (Inlet in Abbildung 5.30) durchgeführt. Das SL(5/5) zeigt kein ausgezeichnetes magnetisches Moment. Die Sättigungsmagnetisierung beträgt $M_{\text {sat, }(5 / 5)}=$ $0,38 \mu_{B} /(\operatorname{Ir}+\mathrm{Mn})$ und aus der temperaturabhängigen Messung lässt sich keine Übergangstemperatur ermitteln.

Für $n=2$ verändert sich der Verlauf der temperaturabhängigen Kurve und zeigt einen ferromagnetischen Übergang bei $T_{C,(2 / 2)}=103 \mathrm{~K}$, was mit den Beobachtungen in [16] übereinstimmt. Das Sättigungsmoment beträgt

${ }^{12}$ Vergleiche Abbildung 2.10 in Kapietel 2.2.1. 

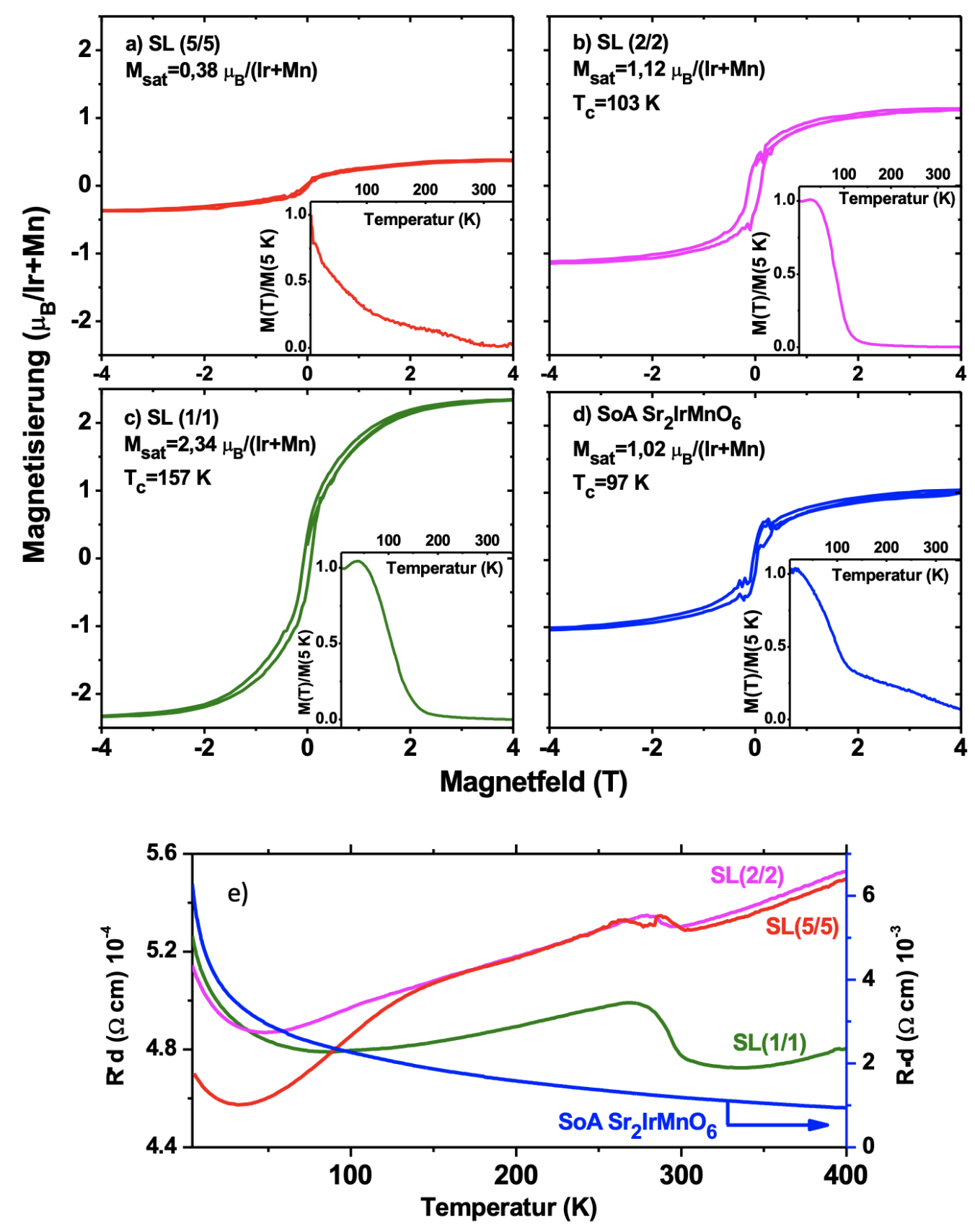

Abbildung 5.30.: a) - d) Feldabhängige Magnetisierungsmessungen der Heterostrukturen $\left(\mathrm{SrIrO}_{3}\right)_{n} /\left(\mathrm{SrMnO}_{3}\right)_{n}$-Proben mit $n=$ 5,2,1 und der SoA-Probe auf $\mathrm{STO}(100)$ bei $T=5 \mathrm{~K}$. Jeweils im Inlet ist die teperaturabhängige Messung bei einem angelegten Feld von $H=100$ Oe zu sehen. e) Temperaturabhängige Messung des spezifischen Flächenwiderstandes der Heterostrukturen $\left(\mathrm{SrIrO}_{3}\right)_{n} /\left(\mathrm{SrMnO}_{3}\right)_{n^{-}}$ Proben mit $n=5,2,1$ und der SoA-Probe auf STO(100). 
$M_{\text {sat },(2 / 2)}=1,12 \mu_{B} /(\operatorname{Ir}+\mathrm{Mn})$ und ist damit um ein Vielfaches gegenüber dem SL(5/5) erhöht. Dieser Trend ist für das SL(1/1) sogar noch stärker ausgeprägt. Die Sättigungsmagnetisierung erhöht sich auf $M_{\text {sat,(1/1) }}=$ $2,34 \mu_{B} /(\mathrm{Ir}+\mathrm{Mn})$ mit einer Übergangstemperatur von $T_{C,(1 / 1)}=157 \mathrm{~K}$. Diese Beobachtungen deuten auf eine ausgeprägte Grenzflächenkopplung hin, die in einer ferromagnetischen Grenzfläche resultiert, die nach [16,17] auf einen Ladungstransfer vom Ir auf das Mn zurückzuführen ist (siehe Kapitel 2.3.2). Die SoA-Probe weist ein Moment von $M_{s a t, S o A}=1,02 \mu_{B} /$ $(\mathrm{Ir}+\mathrm{Mn})$ auf. Das Moment ist damit geringer als für die Probe mit lagenweiser B-Platzordnung und deutet damit auf grundlegende Unterschiede in den jeweiligen Wechselwirkungen zwischen den beteiligten Kationen hin.

Abbildung 5.31 e) zeigt zusammenfassend die Widerstandsmessungen der Heterostrukturen und der SoA-Probe auf STO(100). Für die Heterostrukturen zeigt sich ein charakteristischer Verlauf. Oberhalb der Übergangstemperaturen $T_{C}$ zeigen die Proben metallisches Verhalten mit einem ausgezeichneten Maximum bei $T=290 K(\mathrm{SL}(2 / 2)$ und $\mathrm{SL}(5 / 5))$ und $T=$ $275 K(\mathrm{SL}(1 / 1))$. Die thermische Energie beträgt in dem Temperaturbereich $\approx 0,025 \mathrm{eV}$ und liegt damit im Bereich der Spin-Bahn-Kopplung $\xi$ für $\mathrm{SrMnO}_{3}$ [110]. Das Maximum kann damit auf die Wechselwirkung der $\mathrm{SOC}$ des $\mathrm{SrMnO}_{3}$ und $\mathrm{SrIrO}_{3}$ an der Grenzfläche zurückgeführt werden, die zu einer Erhöhung des Widerstandes führt. Die Ursache kann ebenfalls im Phasenübergang des $\mathrm{SrMnO}_{3}\left(T_{N} \approx 255 \mathrm{~K}\right)$, liegen, der an der Granzfläche zu einer Erhöhung des Widerstandes führt. Unterhalb der Übergangstemperatur ist der Verlauf des Widerstandes nicht mehr linear. Er wird unterhalb von $T \approx 50 \mathrm{~K}$ isolierend. Das isolierende Verhalten bei niedrigen Temperaturen wird mit möglichen Verunreinigungen innerhalb der Probe erklärt. Die SoA-Probe (Blau) hingegen zeigt durchweg ein isolierendes Verhalten und hebt sich damit von den Heterostrukturen deutlich ab. Die Lagenordnung der B-Kationen führt somit zu einem anderen elektrischen Verhalten, das sich von der SoA-Probe mit B-Platzordnung in der NaCl-Anordnung unterscheidet. Die jeweilige Ordnung ruft somit unterschiedliche Wechselwirkungen zwischen der Kationen hervor, die sich neben dem magnetischen Verhalten auch im elektrischen Verhalten widerspiegeln. 
Anomaler Halleffekt Im Rahmen der Bachelorarbeit von Ludwig Scheuchenpflug wurden die Heterostrukturen mit $n=5,2$ und 1 strukturiert und Hallmessungen durchgeführt. Ausführlich können die Ergebnisse in [153] nachvollzogen werden. Im Folgenden sollen kurz die wesentlichen Ergebnisse der Untersuchungen vorgestellt werden, um einen tieferen Einblick in die Grenzflächeneffekte der $\mathrm{SrMnO}_{3} / \mathrm{SrIrO}_{3}$-Proben zu erhalten.

Die Probe mit $n=5$ zeigt keine Anzeichen eines anomalen und topologischen Halleffektes. Dies ist im Einklang mit den Beobachtungen der Magnetisierung und der Literatur [16]. Es bestätigt, dass für die Heterostrukturen mit $n=5$ keine ausgeprägte Grenzflächenkopplung stattfindet.

Für die Proben mit $n=1$ und 2 wird hingegen der anomale Halleffekt beobachtet, der auf die Ausbildung der Grenzflächeneffekte zurückzuführen ist [16]. Abbildung 5.31 zeigt exemplarisch die temperaturabhängige Messung des Hallwiderstandes der SL(1/1) bei $90^{\circ}$ a) (dabei ist die Probenoberfläche parallel zum Magnetfeld ausgerichtet) und $0^{\circ} \mathrm{b}$ ) (dabei ist die Probenoberfläche senkrecht zum Magnetfeld ausgerichtet, wie in Abbil-
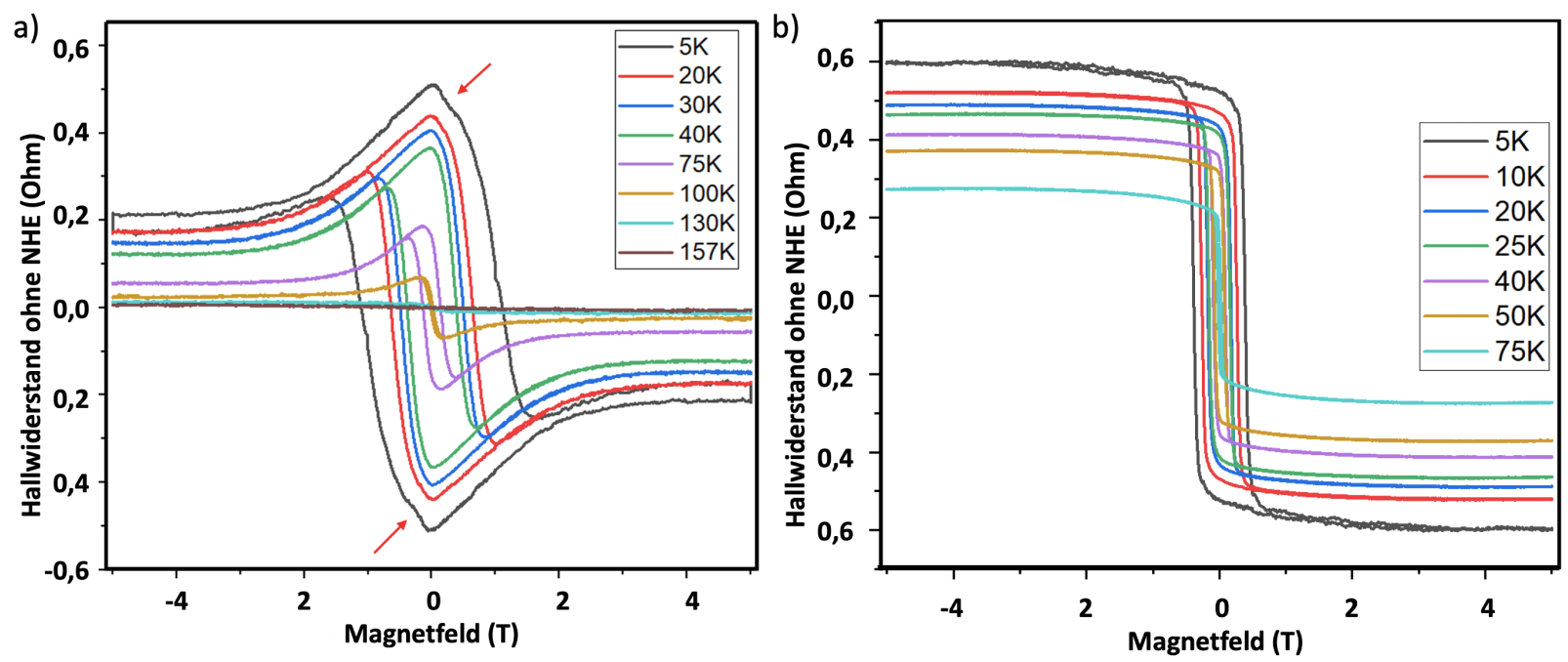

Abbildung 5.31.: Temperaturabhängige Hallmessung der Heterostruktur $\left(\mathrm{SrIrO}_{3}\right)_{n} /\left(\mathrm{SrMnO}_{3}\right)_{n}$ mit $n=1$ bei einem Winkel von a) $90^{\circ}$ und b) $0^{\circ}$. Mit dem roten Pfeil ist die Delle markiert, die Anzeichen auf den topologischen Halleffekt liefert. Die Messung wurde angefertigt von Ludwig Scheuchenpflug an der Universität Augsburg und ist aus seiner Arbeit entnommen [153]. 
dung 2.13 dargestellt). Die Messung in Abbildung 5.31 a) zeigt unterhalb von $T<70 K$ eine Delle, die in einem Winkelbreich von $80^{\circ}$ bis $90^{\circ}$ gefunden werden kann. Sie ist vergleichbar mit dem Signal für den topologischen Halleffekt (vergleiche Abbildung 2.13) und deutet somit auf einen möglichen THE innerhalb der Grenzflächen. Wie in Abbildung 5.31 b) zu erkennen ist, ist diese Delle dort nicht zu sehen. Für die SL(2/2) Probe fallen im selben Winkelbereich ebenfalls Auffälligkeiten auf, da sich die Messungen durch numerische Anpassung nicht mehr angleichen lassen, was auch dort auf den THE deuten lässt.

Zusammenfassung und Diskussion Die strukturellen Untersuchungen an den Heterostrukturen haben ergeben, dass die genaue Anzahl an Einheitszellen pro Monolage nicht ideal getroffen sind. Die Anzahl der Einheitszellen weicht allerdings nicht um mehr als zwei Einheitszellen ab. Innerhalb der Röntgenuntersuchungen zeigen die Proben ausgeprägte Peaks der Überstruktur, die unter anderem durch die starken Unterschiede in den optischen Eigenschaften des $\mathrm{SrIrO}_{3}$ und $\mathrm{SrMnO}_{3}$ zustande kommen. Aufgrund der starken Verspannungszustände der Einzelschichtsysteme auf dem STO-Substrat lassen sich keine geschlossenen bzw. glatten Einzelschichten mit einer Schichtdicke von $d>4$ u.c. wachsen. Dies spiegelt sich im hohen RMS-Wert der (5/5) Probe wider und deckt sich mit der Beobachtung an dem Einzelschichtsystem $\mathrm{SrIrO}_{3}$, das ab einer Schichtdicke von $d_{S I O}=4$ u.c. zu relaxieren beginnt.

Die lagenweise Deposition der Einzelschichtsysteme in (100)-Orientierung hat gezeigt, dass das vorgestellte Konzept der vorherigen Kapitel auch auf andere Orientierungen angewandt werden kann. Erstmals wurde das Doppelperowskit $\mathrm{Sr}_{2} \mathrm{IrMnO}_{6}$ sowohl auf $\mathrm{STO}(100)$ und (111) synthetisiert, zu dem sich bislang keine Literaturberichte finden lassen. Das Wachstum der SoA-Referenzprobe auf STO(111) zeigt einen Überstrukturpeak, der belegt, dass das System natürlich in der NaCl-Struktur die B-Platzordnung ausbildet. Mit der lagenweise Deposition ist es gelungen, die nicht natürliche Lagenordnung künstlich herzustellen. Die vorliegende Ordnung hat ausschlaggebende Auswirkungen auf die magnetischen und elektrischen Eigenschaf- 
ten. Während die SoA-Probe auf $\mathrm{STO}(100)$ isolierend ist und ein geringes magnetisches Moment aufweist, zeigt die LbL-Probe in (100)-Richtung metallisches und ferromagnetisches Verhalten.

Die magnetischen Untersuchungen zeigen, dass der Grenzflächenmagnetismus sich erst für die Probe mit $n \leq 2$ ausbildet. Es findet ein Ladungstransfer von Ir in Mn statt, der auf den Überlapp der benachbarten Orbitale zurückzuführen ist (Molekuhlarorbit-Modell). Um zu untersuchen, ob sich der Ladungstransfer auch in (111)-Orientierung ausbildet, werden im Zuge der vorliegenden Arbeit ebenfalls Untersuchungen am selben Materialsystem auf STO(111) durchgeführt. Die strukturellen, magnetischen und elektrischen Ergebnisse sind in Abbildung A.11 im Anhang zu finden. Die temperaturabhängige Magnetisierungsmessung zeigt einen ferromagnetischen Übergang für sämtliche Proben. Aus der Messung der Sättigungsmagnetisierung ergeben sich jedoch deutlich kleinere Werte $M_{\text {Sat,(111) }}<1 \mu_{B} /(\operatorname{Ir}+\mathrm{Mn})$, die auf eine vergleichsweise schwache ferromagnetische Phase deuten. Im Rahmen des Molekularorbit-Modelles lässt sich diese Beobachtung mit einem geringeren Überlapp der benachbarten Orbitale in (111)-Richtung erklären, der zu einem deutlich schwächeren Ladungstransfer führt.

Für die Proben mit magnetischer Phase $(n=1$ und 2) wird der anomale Halleffekt beobachtet. Winkelabhängige Messungen zeigen in einem Bereich von $80^{\circ}$ bis $90^{\circ}$ unterhalb von $70 \mathrm{~K}$ Anzeichen auf den topologischen Halleffekt. Ob es sich in beiden Fällen tatsächlich um den THE handelt, lässt sich im Rahmen der Untersuchungen allerdings nicht zweifelsfrei klären [153]. Für die SL(1/1) Probe ist das Messsignal zu stark mit dem Magnetowiderstand überlagert. Für das $\mathrm{SL}(2 / 2)$ hingegen ist ungeklärt, ob bisher die Parameter der numerischen Anpassung fehlerhaft sind. Betrachtet man darüber hinaus die Stabilität von Skyrmionen, ist eher davon auszugehen, dass es sich nicht um den THE handelt. Weil Skyrmionen die höchste Stabilität aufweisen, wenn das Feld senkrecht zur Grenzfläche steht, müssten diese bei einem Winkel von $0^{\circ}$ am stärksten ausgeprägt sein. Dies ist hier allerdings bei $90^{\circ}$ der Fall, was der Existenz von Skyrmionen widerspricht [153].

Das Ausbleiben des topologischen Halleffektes kann mehrere Ursachen ha- 
ben. Ein Grund kann in der Symmetrie der Heterostruktur liegen. Sofern sich die Dzyaloshinskii-Moriya-Wechselwirkung an den Grenzflächen ausbildet, kann der resultierende Dzyaloshinskii-Moriya-Vektor $\boldsymbol{D}_{i, j}$ an der $\mathrm{SrIrO}_{3}$ auf $\mathrm{SrMnO}_{3}$ Grenzfläche dem Vektor an der $\mathrm{SrMnO}_{3}$ auf $\mathrm{SrIrO}_{3}$ Grenzfläche entgegengesetzt sein, sodass sich die Wechselwirkung gegenseitig auslöscht. Das Prinzip der asymmetrischen Grenzflächen zur Bildung des topologischen Halleffektes wurde in Grenzflächen zwischen $\mathrm{LaMnO}_{3}$ und $\mathrm{SrIrO}_{3}$ untersucht. Durch den Einbau einer Monolage $\mathrm{SrMnO}_{3}$ an einer der beiden Grenzflächen wird der Effekt des topologischen Halleffektes deutlich gesteigert [20].

Ein weiterer Grund kann in dem intrinsischen antiferromagnetischen Verhalten des $\mathrm{SrMnO}_{3}$ begründet liegen. Zwar entsteht durch die Kopplung an der Grenzfläche zwischen $\mathrm{SrMnO}_{3}$ und $\mathrm{SrIrO}_{3}$ (für $n<3$ ) eine ferromagnetische Phase, jedoch ist nicht ausgeschlossen, dass das 3d-System zur Bildung des topologischen Halleffektes einen intrinsischen Ferromagnetismus zur Bildung der Dzyaloshinskii-Moriya-Wechselwirkung mitbringen muss.

Um diesen Vermutungen nachzugehen, werden im Rahmen der vorliegenden Arbeit weitere Heterostrukturen hergestellt, die im Folgenden vorgestellt werden sollen. Die Untersuchungen zum topologischen Halleffekt sind zum Zeitpunkt der vorliegenden Arbeit jedoch noch nicht abgeschlossen, weshalb lediglich die strukturellen und magnetischen Eigenschaften vorgestellt werden können. 


\subsubsection{Grenzflächeneffekte in $\mathrm{La}_{0.7} \mathrm{Sr}_{0.3} \mathrm{MnO}_{3} / \mathrm{SrlrO}_{3^{-}}$ Heterostrukturen}

Um zu überprüfen, ob der Ferromagnetismus zur Bildung des topologischen Halleffektes notwendig ist, werden Heterostrukturen aus $\left(\mathrm{La}_{0.7} \mathrm{Sr}_{0.3} \mathrm{MnO}_{3}\right)_{n} /$ $\left(\mathrm{SrIrO}_{3}\right)_{2}$ mit $n=6$ und $4(\mathrm{SL}(6 / 2)$ und $\mathrm{SL}(4 / 2))$ auf STO (100) hergestellt und auf ihre strukturellen, elektrischen und magnetischen Eigenschaften hin untersucht. Bisherige Untersuchungen haben an Bilagen aus $\mathrm{La}_{1-x} \mathrm{Sr}_{x} \mathrm{MnO}_{3}$ mit $x=0,3$ und $\mathrm{SrIrO}_{3}$ den topologischen Halleffekt nachweisen können [21]. Die folgenden Untersuchungen sollen zeigen, ob sich die Beobachtung auch auf Heterostrukturen übertragen lässt, die keine asymmetrischen Grenzflächen aufweisen.

Das Wachstum des Einzelschichtsystems $\mathrm{SrIrO}_{3}$ und die Heterostrukturen aus $\mathrm{SrMnO}_{3}$ und $\mathrm{SrIrO}_{3}$ haben gezeigt, dass $\mathrm{SrIrO}_{3}$ insbesondere bei kleinen Schichtdicken $d_{S I O} \leq 2$ u.c. stabil wächst. Aus diesem Grund wird für die folgenden Probe die Schichtdicke des $\mathrm{SrIrO}_{3}$ mit $d_{S I O}=2$ u.c. konstant gehalten. Um eine hohe Qualität der Grenzflächen zu gewährleisten, werden die Proben aus lediglich sechs Wiederholungen der Bilagen hergestellt. Zusätzlich wird eine $\mathrm{La}_{0.7} \mathrm{Sr}_{0.3} \mathrm{MnO}_{3}$-Probe auf $\mathrm{STO}(100)$ mit $d_{L S M O}=11,7 \mathrm{~nm}$ als Referenzprobe für das $\mathrm{La}_{0.7} \mathrm{Sr}_{0.3} \mathrm{MnO}_{3}$ innerhalb der Heterostrukturen hergestellt und untersucht.

Strukturelle Eigenschaften Abbildung 5.32 zeigt die Ergebnisse der strukturellen und elektrischen Untersuchungen an den Heterostrukturen und der $\mathrm{La}_{0.7} \mathrm{Sr}_{0.3} \mathrm{MnO}_{3}$-Referenzprobe. Die XRD-Messungen zeigen stark ausgeprägte Peaks, die auf die Überstruktur zurückzuführen sind und die hohe Qualität der Proben und deren Grenzfläche hervorheben. Numerische Anpassungen an den XRR-Messungen sind in Abbildung 5.32 b) zu sehen, aus denen sich die jeweilige Schichtdicke der Monolagen ergibt. Für die Probe $(6 / 2)$ ergibt sich für die Einzelschicht $\mathrm{La}_{0.7} \mathrm{Sr}_{0.3} \mathrm{MnO}_{3}$ eine Dicke von $d_{L S M O}=2,63 \mathrm{~nm}=6,8$ u.c., womit diese dicker ausfällt als erwünscht. Mit $d_{L S M O}=1,69 \mathrm{~nm}=4,4$ u.c. wird die Schichtdicke für die (4/2) Probe genauer erreicht. 

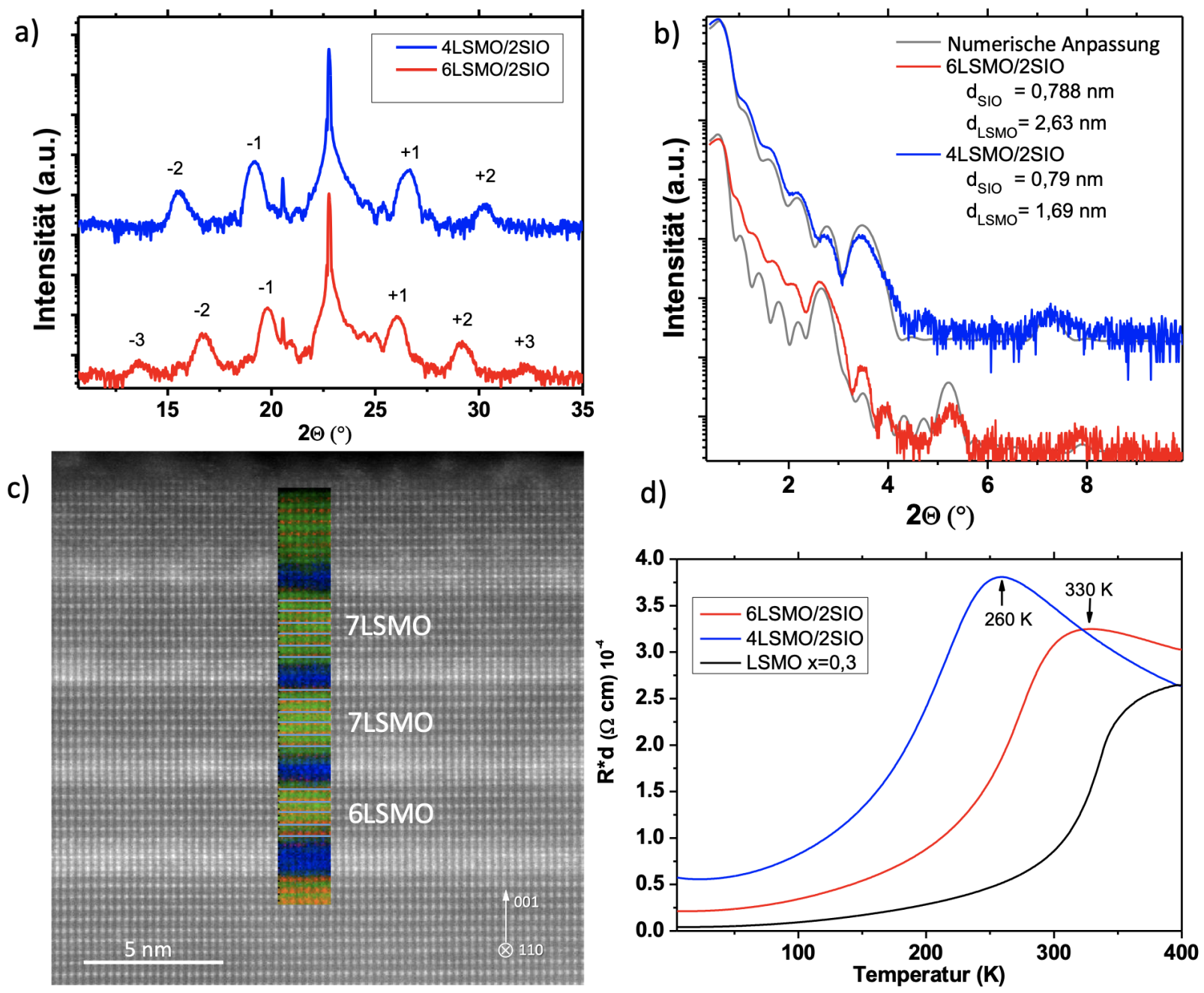

Abbildung 5.32.: Strukturelle und elektrische Untersuchungen an den $\left(\mathrm{La}_{0.7} \mathrm{Sr}_{0.3} \mathrm{MnO}_{3}\right)_{n} /\left(\mathrm{SrIrO}_{3}\right)_{2}$ mit $n=6$ (Rot) und 4 (Blau) und der $\mathrm{La}_{0.7} \mathrm{Sr}_{0.3} \mathrm{MnO}_{3}$ Probe mit $d=11,7 \mathrm{~nm}$ (Schwarz). a) XRD-Messung, b) XRR Messungen mit numerischen Anpassungen, c) HAADF STEM-Aufnahme mit EELS-Mapping der Heterostruktur mit $\mathrm{n}=6$ (angefertigt von Vladimir Roddatis) und d) Messung des spezifischen Flächenwiderstandes.

Die TEM-Aufnahme in Abbildung 5.32 c) bestätigt diese Ergebnisse, in dem die einzelnen $\mathrm{La}_{0.7} \mathrm{Sr}_{0.3} \mathrm{MnO}_{3}$-Monolagen hervorgehoben sind. Es ist $\mathrm{zu}$ erkennen, dass für SL(6/2) Einzellagen aus sechs und sieben Einheitszellen vorliegen. Darüber hinaus werden scharfe Grenzflächen beobachtet, die die starken Überstrukturpeaks der XRD-Messung erklären. 

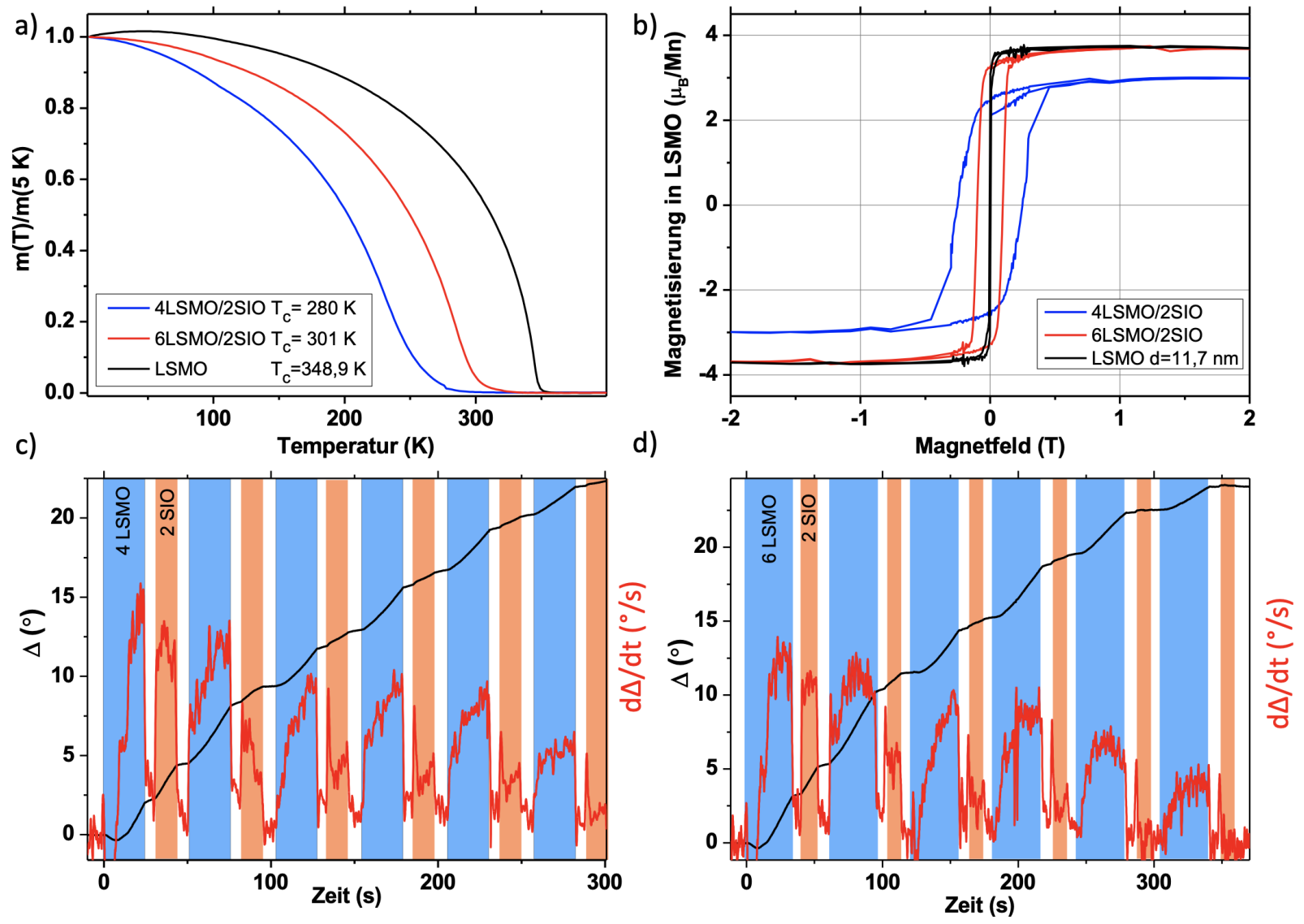

Abbildung 5.33.: Magnetischen Untersuchungen an den $\left(\mathrm{La}_{0.7} \mathrm{Sr}_{0.3} \mathrm{MnO}_{3}\right)_{n} /$ $\left(\mathrm{SrIrO}_{3}\right)_{2}$ mit $n=6$ (Rot) und 4 (Blau) und der $\mathrm{La}_{0.7} \mathrm{Sr}_{0.3} \mathrm{MnO}_{3}$-Referenzprobe mit $d=11,7 \mathrm{~nm}$ (Schwarz) auf STO(100). a) Temperaturabhängige und b) feldabhängige Messung der Magnetisierung. c) und d) die in-situEllipsometrie-Messung der Heterostrukturen (Schwarz) und die zeitliche Ableitung der Phasenverschiebung (Rot).

Elektrische und magnetische Eigenschaften Die Einzelschichtdicke des $\mathrm{La}_{0.7} \mathrm{Sr}_{0.3} \mathrm{MnO}_{3}$ wirkt sich auf die elektrischen und magnetischen Eigenschaften der Heterostrukturen aus, wie in Abbildung 5.32 d) und 5.33 a,b) zu erkennen ist. Verglichen mit der $\mathrm{La}_{0.7} \mathrm{Sr}_{0.3} \mathrm{MnO}_{3}$-Referenzprobe sinkt die Übergangstemperatur mit abnehmender Schichtdicke für $\mathrm{La}_{0.7} \mathrm{Sr}_{0.3} \mathrm{MnO}_{3}$. So beträgt die Übergangstemperatur für die (6/2) Probe $T_{C}=301 \mathrm{~K}$ und für die $(4 / 2)$ Probe $T_{C}=280 K$. Das Maximum der Widerstandsmessung folgt dem selben Trend und nimmt mit abnehmender Schichtdicke $d_{L S M O}$ ebenfalls ab. Aus Abbildung 5.33 b) wird entnommen, dass auch das ma- 
gnetische Moment der $\mathrm{La}_{0.7} \mathrm{Sr}_{0.3} \mathrm{MnO}_{3}$-Schicht mit sinkender Einzelschichtdicke abnimmt und seine Eigenschaften als Einzelschichtsystem zunehmend verliert. Bemerkenswert ist, dass für die $\mathrm{La}_{0.7} \mathrm{Sr}_{0.3} \mathrm{MnO}_{3}$ Einzelschicht ein Koerzitivfeld von $H_{c}=34 O e$ wohingegen für die Heterostruktur mit $n=4$ ein Koerzitivfeld von $H_{c,(4 / 2)}=1000 \mathrm{Oe}$ und für $n=6 H_{c,(6 / 2)}=2500 \mathrm{Oe}$ gemessen wird. Dies deutet auf eine starke Kopplung des magnetischen Momentes des $\mathrm{La}_{0.7} \mathrm{Sr}_{0.3} \mathrm{MnO}_{3}$ an der Grenzfläche.

In-situ-Ellipsometrie Abbildung 5.33 c) und d) zeigen die Phasenverschiebung $\Delta$ und deren zeitliche Ableitung der Ellipsometrie-Messung der jeweiligen Heterostrukturen. Für beide Proben stellt sich bereits nach der ersten Monolage ein charakteristischer Verlauf des Messsignales ein. Die zeitliche Ableitung des Phasenwinkels zeigt für beide Proben innerhalb der Deposition für $\mathrm{La}_{0.7} \mathrm{Sr}_{0.3} \mathrm{MnO}_{3}$ für die ersten beiden Einheitszellen einen Anstieg, der anschließend abflacht. Wie bereits im Kapitel für $\mathrm{LaMnO}_{3}$ und $\mathrm{LaNiO}_{3}$ beschrieben, ist das Ellipsometriesignal empfindlich auf elektrische Veränderungen innerhalb des Materials. In Verbindung mit den beobachteten hohen Koerzitivfeldern kann dies auf eine Kopplung an der $\mathrm{La}_{0.7} \mathrm{Sr}_{0.3} \mathrm{MnO}_{3}$ auf $\mathrm{SrIrO}_{3}$ Grenzfläche hindeuten.

Zusammenfassung und Diskussion Die strukturellen Untersuchungen zeigen, dass die Heterostrukturen von hoher Qualität mit scharfen Grenzflächen sind. Lediglich die Einzelschichtdicke für $\mathrm{La}_{0.7} \mathrm{Sr}_{0.3} \mathrm{MnO}_{3}$ fällt für die Probe mit $n=6$ höher aus und entspricht damit mehr einer $(7 / 2)$ Probe. Die elektrischen und magnetischen Eigenschaften deuten in Verbindung mit Ellipsometrie-Messungen auf eine Kopplung an den Grenzflächen hin. Zukünftige Untersuchungen an den Proben werden Aufschluss darüber geben, ob der topologische Halleffekt in den Probensystemen zu beobachten sein wird. Neuste Untersuchungen an Bilayern aus $\mathrm{La}_{0.7} \mathrm{Sr}_{0.3} \mathrm{MnO}_{3}$ und $\mathrm{SrIrO}_{3}$ deuten dabei auf vielversprechende Ergebnisse hin. Diese haben den topologischen Halleffekt an der Grenzfläche zwischen $\mathrm{La}_{0.7} \mathrm{Sr}_{0.3} \mathrm{MnO}_{3}$ und $\mathrm{SrIrO}_{3}$ beobachten können [21]. Die Gruppe um Y. Li hat dabei mittels PLD La $\mathrm{La}_{0.7} \mathrm{Sr}_{0.3} \mathrm{MnO}_{3}$ mit der Dicke von acht Einheitszellen, $d_{L S M O}=8$ u.c., 
auf zwei Einheitszellen $\mathrm{SrIrO}_{3}, d_{S I O}=2$ u.c., auf STO(100) hergestellt. Der topologische Halleffekt ist dabei bei Temperaturen bis zu $200 \mathrm{~K}$ zu beobachten, der mit einer nicht koplanaren (NC) Spintextur in Verbindung gebracht wird, der in das $\mathrm{La}_{0.7} \mathrm{Sr}_{0.3} \mathrm{MnO}_{3}$ induziert wird [21].

Darüber hinaus betrachtete die Gruppe um L. Bergmann [183] das selbe Bilagensystem und untersucht den Einfluss der Sequenz der Grenzfläche. Dafür werden mittels PLD sowohl Proben hergestellt, in denen $\mathrm{SrIrO}_{3}$ auf $\mathrm{La}_{0.7} \mathrm{Sr}_{0.3} \mathrm{MnO}_{3}$ (S1) gewachsen wird, als auch $\mathrm{La}_{0.7} \mathrm{Sr}_{0.3} \mathrm{MnO}_{3}$ auf $\mathrm{SrIrO}_{3}$ (S2). Die Einzelschichtdicken betragen jeweils $d_{S I O}=8$ u.c. und $d_{L S M O}=$ 12 u.c. und sind auf STO(100) hergestellt. Messungen der Magnetisierung und feldabhängige XMCD-Messungen der Mn- $\mathrm{L}_{2,3}$-Kante zeigen eine Veränderung in der magnetischen Ordnung des $\mathrm{La}_{0.7} \mathrm{Sr}_{0.3} \mathrm{MnO}_{3}$, die für die S2Probe größer ausfällt, als für die S1-Probe. Die Grenzflächen unterscheiden sich somit voneinander. Der Einfluss der Wachstumssequenz wird auf die Grenzflächengitterstruktur zurückgeführt, von der bekannt ist, dass sie die magnetische Grenzflächenkopplung beeinflusst, die durch oktaedrische $\mathrm{MnO}_{6}$-Rotationen und/oder Verzerrungen bestimmt wird [183].

Es liegt somit nahe, dass die unterschiedlichen Grenzflächen zu unterschiedlich stark ausgeprägten Dzyaloshinskii-Moriya-Wechselwirkungen führen, sodass sich die Dzyaloshinskii-Moriya-Vektoren nicht gegenseitig auslöschen. Damit wäre der topologische Halleffekt an den hergestellten Heterostrukturen messbar. 
Kapitel

\section{Zusammenfassung und Ausblick}

Zusammenfassung der Ergebnisse Über die lagenweise Deposition ausgewählter Einzelschichtsysteme wurden Versuche durchgeführt, die B-Platzordnung künstlich hervorzurufen. Am Materialsystem $\mathrm{LaNiO}_{3} / \mathrm{LaMnO}_{3}$ wurde dabei der Ansatz gewählt, die Einzelschichtdicke systematisch zu reduzieren, um so die genaue Depositionsmenge für eine Einzellage zu treffen. Auf diese Weise ließen sich neben der erzielten Kationordnung auch die Grenzflächeneffekte untersuchen. Mit Hilfe der in-situ-Ellipsometrie ist es gelungen einen direkten Einblick in den Ladungstransfer zwischen Mn und $\mathrm{Ni} \mathrm{zu}$ erlangen und dessen charakteristische Länge direkt abschätzen. Mittels Raman-Spektroskopie wurde der kontinuierliche strukturelle Übergang zur Doppelperowskitphase beobachtet. Erstmals konnten magnetische Messungen die Doppelperowskitphase an der Grenzfläche zwischen den beiden Systemen nachweisen. Diese Beobachtung hebt die Wachstumsbedingungen der MAD gegenüber den konventionellen Wachstumsmethoden hervor. Die hoch energetische Flüssig/Gas-Phase in Verbindung mit dem hohen Sauerstoffpartialdruck beim Wachstum müssen die Ausbildung der DP-Phase begünstigen. Außerdem zeigen die Messungen eine Asymmetrie zwischen den Grenzflächen. Die Grenzflächeneffekte sind somit direkt an die Sequenz der Grenzfläche gekoppelt. Dies hat einen direkten Einfluss auf die lagenweise Deposition der Einzelschichtsysteme. Weil das $\mathrm{LaNiO}_{3}$ auf $\mathrm{LaMnO}_{3}$ eine raue Grenzfläche bildet, ist es nicht gelungen, eine hohe künstliche BPlatzordnung im LbL-Modus zu erhalten. 
Anschließend wurde das Prinzip auf das System $\mathrm{LaCoO}_{3} / \mathrm{LaMnO}_{3}$ angewandt. Anders als zuvor wurde hier die LbL-Probe direkt aus den Erkenntnissen der Wachstumsbedingungen für die Einzelschichtsysteme heraus hergestellt. Strukturelle Untersuchungen deuteten auf ein fehlerhaftes stöchiometrisches Verhältnis hin. Durch entsprechende Korrektur der Depositionsmengen ist es gelungen, eine LbL-Probe herzustellen, die einen bisher noch nicht erreichten Ordnungsgrad vorweist. Dabei hat sich gezeigt, dass das System sehr empfindlich auf stöchiometrische Änderungen reagiert. Das Einzelschichtsystem $\mathrm{LaCoO}_{3}$ beginnt bereits ab einer Dicke von $d_{L C O}>1$ u.c. seine strukturellen Eigenschaften auf das System zu übertragen. Das Ausbleiben eines ausgeprägten Überstrukturpeaks innerhalb der optimalen LbL-Probe kann jedoch nicht eindeutig geklärt werden. Die gängige Begründung, dass die Antiphasengrenzen den Überstrukturpeak auslöschen scheint nicht zutreffend zu sein, da die lagenweise Deposition der Einzelschichtsysteme die Ausbildung der APB verhindern sollte.

Das Vorgehen wurde auf das System aus $\mathrm{SrTiO}_{3} / \mathrm{SrRuO}_{3}$ erweitert. Die $\mathrm{Sr}_{2} \mathrm{RuTiO}_{6}$-Referenzprobe zeigte eine hohe Intensität des Überstrukturpeaks. TEM-Untersuchungen stimmen mit den Röntgenuntersuchungen überein und deuten auf eine B-Platzordnung von ca. 60 \% hin. Mit den TEM-Bildern ist es gelungen, die Bereiche mit hoher B-Platzordnung sichtbar zu machen. Dabei zeigte sich, dass es Domänen mit hoher B-Platzordnung gibt, die durch Antiphasengrenzen voneinander getrennt sind. EELS-Spektren des Titans deuten sowohl auf die Anwesenheit von $\mathrm{Ti}^{3+}$ als auch auf $\mathrm{Ti}^{4+}$ hin. Die genauen Einblicke in die SoA-Probe lieferten ein tiefgehendes Verständnis für das Wachstum aus einer Lösung heraus, sodass das Wachstum der B-Platz-geordneten Doppelperowskite modelliert werden konnte. Dabei stellte sich heraus, dass der Ladungstransfer eine entschiedene Rolle für die Ausbildung der Ordnung hat. Da Titan die 4+ Valenz stark favorisiert, benötigt es eine hohe Energie innerhalb der Flüssig/Gas-Phase während des MAD-Wachstums, um den Ladungstransfer auszulösen. Durch den Valenzunterschied erhöht sich ebenfalls der Unterschied zwischen den Radien der beteiligten Kationen. Es folgt wahrscheinlich ein selbst verstärkender Effekt, der die Ausbildung der B-Platzordnung unterstützt. 
Den Abschluss der Arbeit bildet die Synthese der 3d/5d-Grenzflächen mit $\mathrm{SrIrO}_{3}$. Die lagenweise Deposition wurde damit im Rahmen der Arbeit erstmals in (100)-Richtung erprobt. Zudem wurden $\mathrm{Sr}_{2} \mathrm{IrMnO}_{6}$-Referenzproben auf STO in (111)- und (100)-Richtung hergestellt, die aus einer Lösung heraus gewachsen wurden. Die Untersuchung der beiden Proben zeigte, dass das System natürlich in der NaCl-Struktur ordnet, das bisher nicht noch nicht beobachtet wurde. Das LbL-Wachstum erzeugt in (100)-Richtung eine Lagenweise Ordnung der B-Kationen, die natürlich für das System nicht vorkommt. Die Probe weist dabei eine ausgeprägte ferromagnetische Phase auf mit vergleichsweise hoher Magnetisierung. Erklärt wird diese Phase durch einen Ladungstransfer im Rahmen des Molekularorbital-Modells. Der Überlapp der benachbarten $3 z^{3}-r^{2}$ Orbitale des Mn und Ir führt zu einer Absenkung des bindenden Orbitales unterhalb des Fermi-Niveaus, wodurch der Ladungstransfer ermöglicht wird. Daraus entsteht schließlich die ferromagnetische Phase. Untersuchungen haben gezeigt, dass sich diese Phase auch in (111)-Orientierung ausbildet. Jedoch muss der strukturelle Überlapp der Orbitale entlang der (111)-Richtung geringer sein, da das magnetische Moment und somit die Stärke der Kopplung deutlich geringer ausfällt.

Die Untersuchungen an $\mathrm{SrIrO}_{3} / \mathrm{SrMnO}_{3}$ weisen den anomalen Halleffekt auf, jedoch keinen eindeutigen topologischen Halleffekt. Die Gründe dafür können durch die antiferromagnetischen Eigenschaften des $\mathrm{SrMnO}_{3}$ begründet sein. Möglich ist es auch, dass der Grund in der Symmetrie der Grenzflächen liegt, wodurch der Dzyaloshinskii-Moriya-Vektor durch die entgegengesetzte Grenzfläche ausgelöscht wird.

Deshalb wurden zusätzlich Heterostrukturen aus $\mathrm{La}_{0.7} \mathrm{Sr}_{0.3} \mathrm{MnO}_{3} / \mathrm{SrIrO}_{3}$ hergestellt. Die Untersuchungen deuten auf eine starke Grenzflächenkopplung hin. Da bereits in Bilagen aus $\mathrm{SrIrO}_{3}$ und $\mathrm{La}_{0.7} \mathrm{Sr}_{0.3} \mathrm{MnO}_{3}$ der topologische Halleffekt nachgewiesen wurde, bildet das Materialsystem damit einen vielversprechenden Kandidaten für die Untersuchung des topologischen Halleffektes, die in Zukunft an der Universität in Augsburg durchgeführt werden. 
Ausblick Die Untersuchungen an dem Doppelperowskit $\mathrm{La}_{2} \mathrm{CoMnO}_{6}$ und dem LbL-System der Einzelschichten $\mathrm{LaCoO}_{3} / \mathrm{LaMnO}_{3}$ haben gezeigt, dass die Beobachtung des Überstrukturpeaks komplexer ist, als bisher angenommen. Die Abweichung der theoretisch berechneten Intensitätsverhältnisse von den experimentellen Werten deutet darauf hin, dass weitere Faktoren Einfluss auf den Peak nehmen, der allein über den Strukturfaktor nicht mit einbezogen wird. Aufschluss über dieses Phänomen können strukturelle Untersuchungen an den $\mathrm{A}_{2} \mathrm{CoMnO}_{6}$ mit $\mathrm{A}=\mathrm{La}, \mathrm{Pr}, \mathrm{Nd}$, Sm, Gd und Y liefern. So zeigt beispielsweise das System mit $\mathrm{A}=\mathrm{Nd}$ einen auffällig starken Peak, der mit der Besetzung des A-Platzes und starken Verkippungen der $\mathrm{MnO}_{6}{ }^{-}$ Oktaeder in Zusammenhang steht.

Darüber hinaus bleibt ungeklärt, weshalb die Überstruktur in den LbLProben des $\mathrm{SrTiO}_{3} / \mathrm{SrRuO}_{3}$ nicht beobachtet wird. TEM- und EELS-Untersuchungen können darüber Aufschluss bieten. Zusätzlich bleibt die Frage ungeklärt, ob trotz der hohen Stabilität des $\mathrm{Ti}^{4+}$ der Ladungstransfer bei der Deposition der Einzelschichtsysteme stattfindet. Dies würde die lagenweise Wachstumsmethode gegenüber dem Wachstum aus einer Lösung heraus weiter hervorheben. Es würde das vorgestellte Wachstumsmodell der geordneten Doppelperowskitphase bestätigen, in dem die hoch energetische Flüssig/Gas-Phase beim Wachstum mittels MAD als Auslöser für den Ladungstransfer und somit für die Ordnung gilt.

Vielversprechende und spannende Erkenntnisse werden im Zusammenhang mit den Heterostrukturen mit $\mathrm{SrIrO}_{3}$ erwartet. Wie bereits erwähnt, bildet die Heterostruktur aus $\mathrm{La}_{0.7} \mathrm{Sr}_{0.3} \mathrm{MnO}_{3} / \mathrm{SrIrO}_{3}$ die Grundlage für die Untersuchungen des topologischen Halleffektes.

Darüber hinaus lassen sich auch am System aus $\mathrm{SrMnO}_{3}$ und $\mathrm{SrMnO}_{3}$ weitere Versuche durchführen. So kann beispielsweise der Einbau einer künstlichen asymmetrischen Grenzflächenabfolge dazu beitragen, den THE zu beobachten. Die Herstellung derartiger Heterostrukturen sind bereits mit der MAD durchgeführt worden. In Heterostrukturen aus $\left(\left(\mathrm{SrTiO}_{3}\right)_{2} /\left(\mathrm{SrIrO}_{3}\right)_{3} /\right.$ $\left.\left(\mathrm{SrMnO}_{3}\right)_{3}\right)_{5}$ wird über den Einbau von $\mathrm{SrTiO}_{3}$ an der Grenzfläche $\mathrm{SrMnO}_{3}$ auf $\mathrm{SrIrO}_{3}$ eine Asymmetrie hergestellt. Auch diese Proben werden an der Universität Augsburg auf den topologischen Halleffekt hin untersucht. 
Die vorliegende Arbeit hat gezeigt, dass über das Wachstum mittels MADDoppelperowskite in hoher kristalliner Qualität aufgewachsen werden können, die einen hohen Grad der B-Platzordnung aufweisen. Da die vorgestellten DP ferromagnetisches Verhalten mit hoher Übergangstemperatur zeigen, können für zukünftige Forschungsunternehmungen daher auch diese mit dem 5d-System $\mathrm{SrIrO}_{3}$ verbunden werden. Dafür sind zum Abschluss der Arbeit erste Versuche durchgeführt worden, in dem das vorgestellte Doppelperowskit $\mathrm{La}_{2} \mathrm{NiMnO}_{6}$ mit $\mathrm{SrIrO}_{3}$ kombiniert wird. Es werden Heterostrukturen in Form von $\left(\left(\mathrm{La}_{2} \mathrm{NiMnO}_{6}\right)_{n} /\left(\mathrm{SrIrO}_{3}\right)_{2}\right)_{6}$ mit $n=6$ und 10 auf STO(100) hergestellt, die insbesondere durch die hohe strukturelle Qualität hervorstechen. Abbildung 6.1 zeigt die XRR- und XRD-Messung der beiden Proben. In beiden Fällen sind ausgeprägte Überstrukturpeaks zu erkennen, die die hohe kristalline Qualität widerspiegeln. Auch die jeweiligen Einzelschichtdicken werden sehr präzise getroffen. Dies wird durch ergänzende TEM-Untersuchungen bestätigt, wie in Abbildung A.12 im Anhang $\mathrm{zu}$ entnehmen ist.
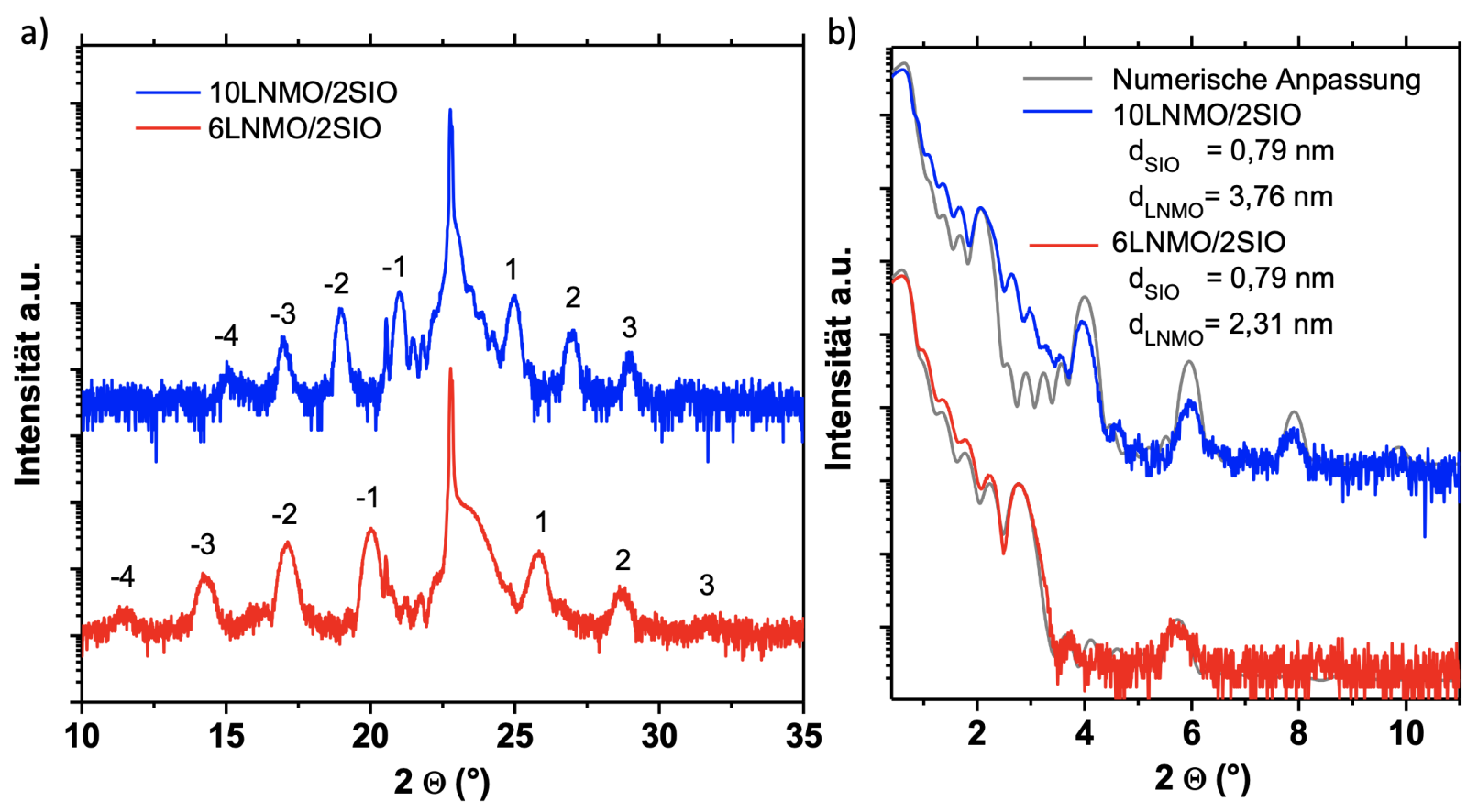

Abbildung 6.1.: a) XRD-Messung und b) XRR-Messung mit numerischer Anpassung der $\left(\left(\mathrm{La}_{2} \mathrm{NiMnO}_{6}\right)_{n} /\left(\mathrm{SrIrO}_{3}\right)_{2}\right)_{6}$ mit $n=6$ und 10 Heterostrukturen. 


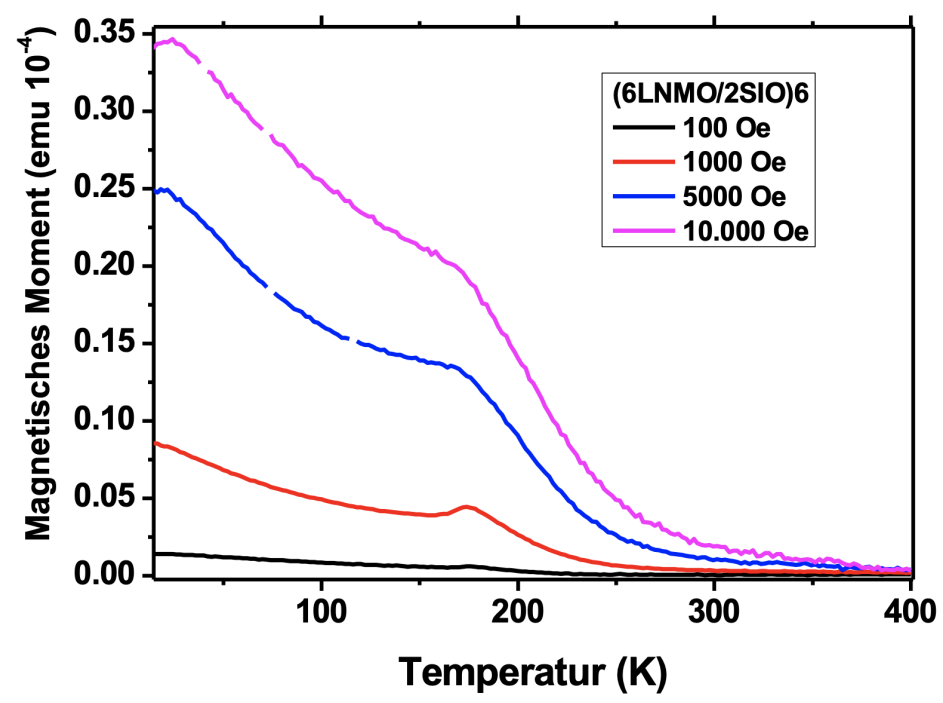

Abbildung 6.2.: Temperaturabhängige Messung der Magnetisierung des $\left(\left(\mathrm{La}_{2} \mathrm{NiMnO}_{6}\right)_{n} /\left(\mathrm{SrIrO}_{3}\right)_{2}\right)_{6}$ mit $n=6$ bei unterschiedlich angelegten Feldern.

Aufgrund des stark isolierenden Verhaltens des Doppelperowskit, lassen sich jedoch keine Untersuchungen nach dem topologischen Halleffekt durchführen. Allerdings zeigen die Magnetisierungsmessungen für die Heterostruktur mit $n=6$ Anzeichen der Grenzflächenkopplung zwischen $\mathrm{La}_{2} \mathrm{NiMnO}_{6}$ und $\mathrm{SrIrO}_{3}$. Bei einem extern angelegten Feld unterhalb von $B \leq 0.5 T$ weist die Kurve einen lokales Maximum bei $T=180 K$ auf. Für $n=10$ wird der Effekt nicht mehr beobachtet, wohingegen die Einzelschichtdicke keinen Einfluss auf das magnetische Moment des DP nimmt, wie in Abbildung A.13 im Anhang zu erkennen ist.

Es gilt diesen Grenzflächeneffekt weiter zu untersuchen. Er zeigt, dass die beiden Systeme an deren Grenzfläche wechselwirken. Wenn es gelingt, ein Doppelperowskit mit geringem Widerstand herzustellen, können auch diese Heterostrukturen vielversprechende Kandidaten für die Untersuchung des topologischen Halleffektes bilden, die mit der MAD zuverlässig in hoher kristalliner Qualität hergestellt werden können. 


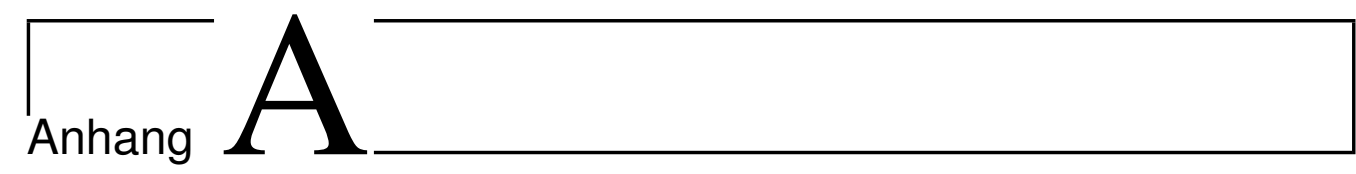

\section{Anhang}

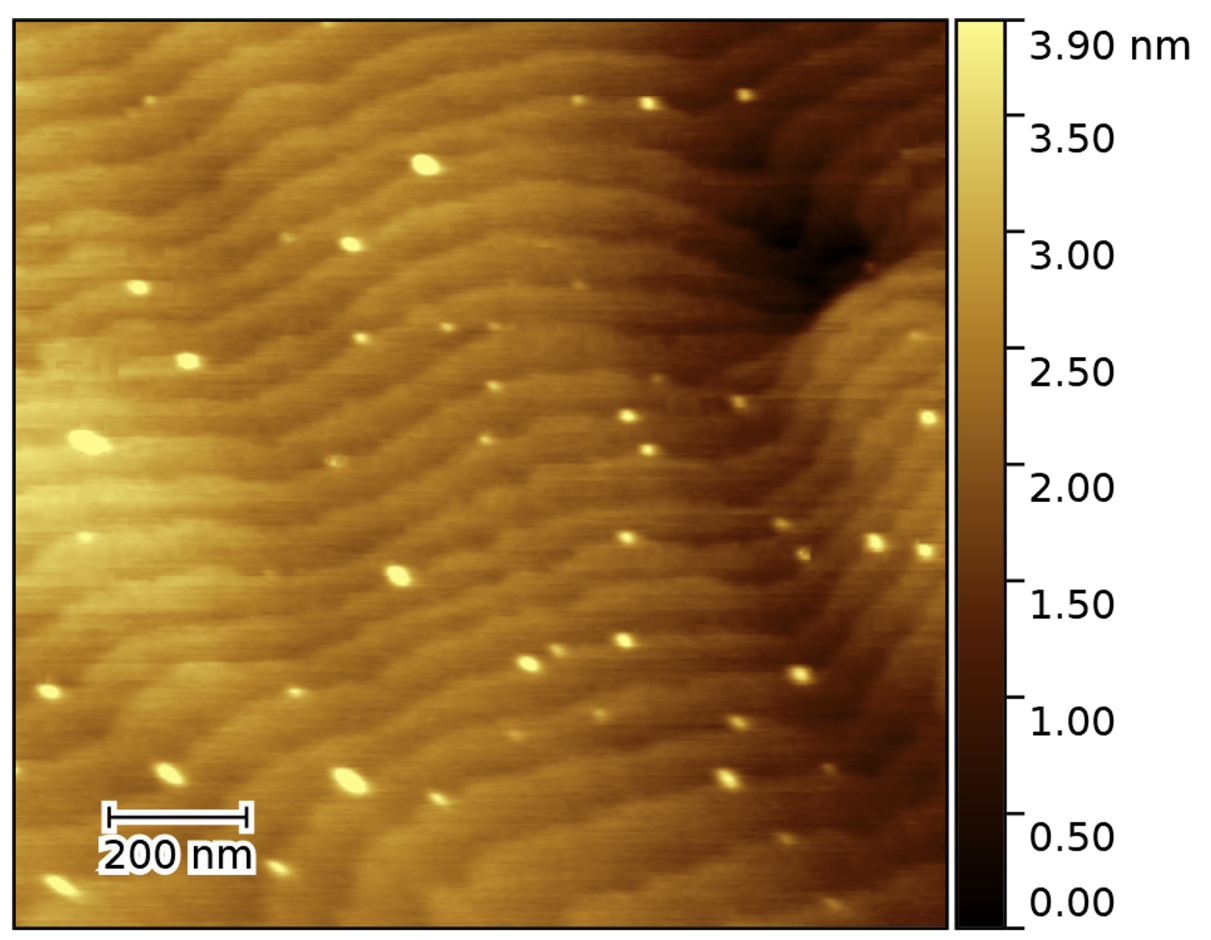

Abbildung A.1.: AFM-Aufnahme einer $\mathrm{La}_{2} \mathrm{CoMnO}_{6}$-Probe auf $\mathrm{STO}(100)$, die unter Unterdruck mittels MAD hergestellt wurde. 


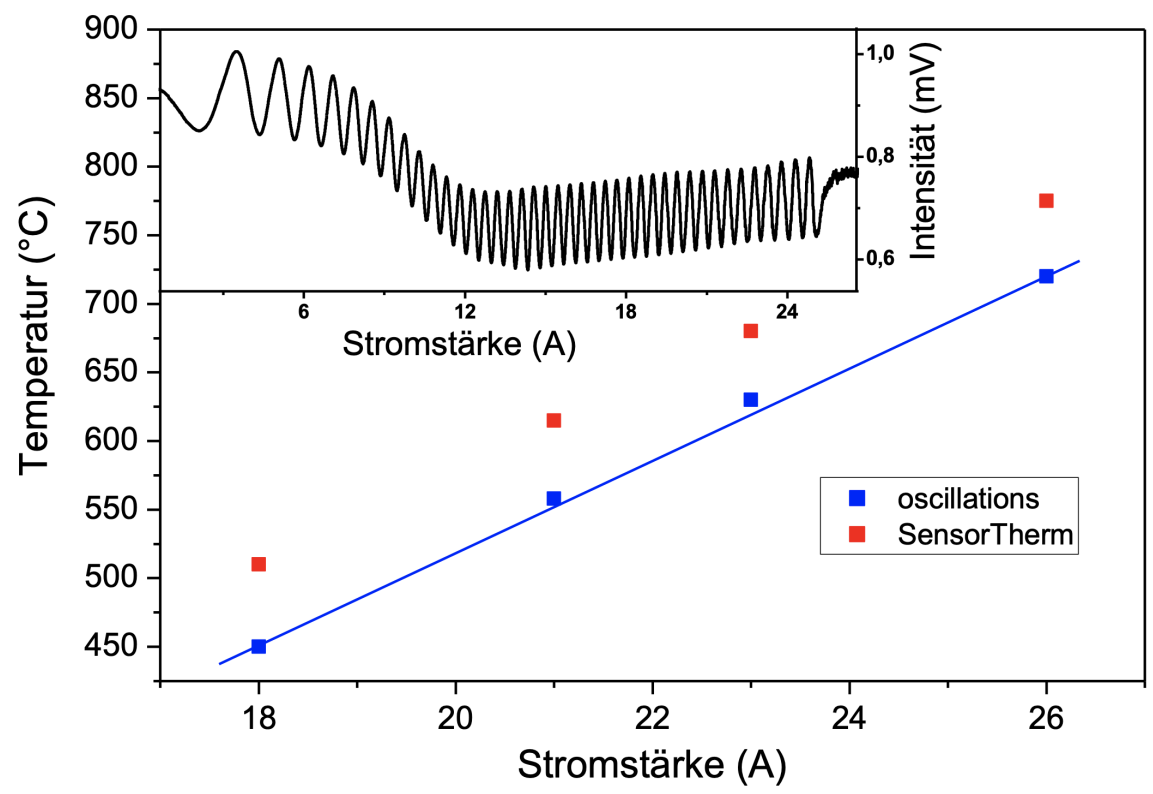

Abbildung A.2.: Temperatur der Substratoberfläche in Abhängigkeit vom Heizstrom. Zwischen dem Pyrometer und der Messung über die Reflexion am Saphir besteht ein Unterschied von bis zu $50 \mathrm{~K}$, der auf eine Fehleinstellung/-eichung des Pyrometers zurückzuführen ist. Im Inlet sind die Intensitätsoszillationen am Saphir dargestellt. Die Messungen wurden von Oleg Shapoval durchgeführt. 


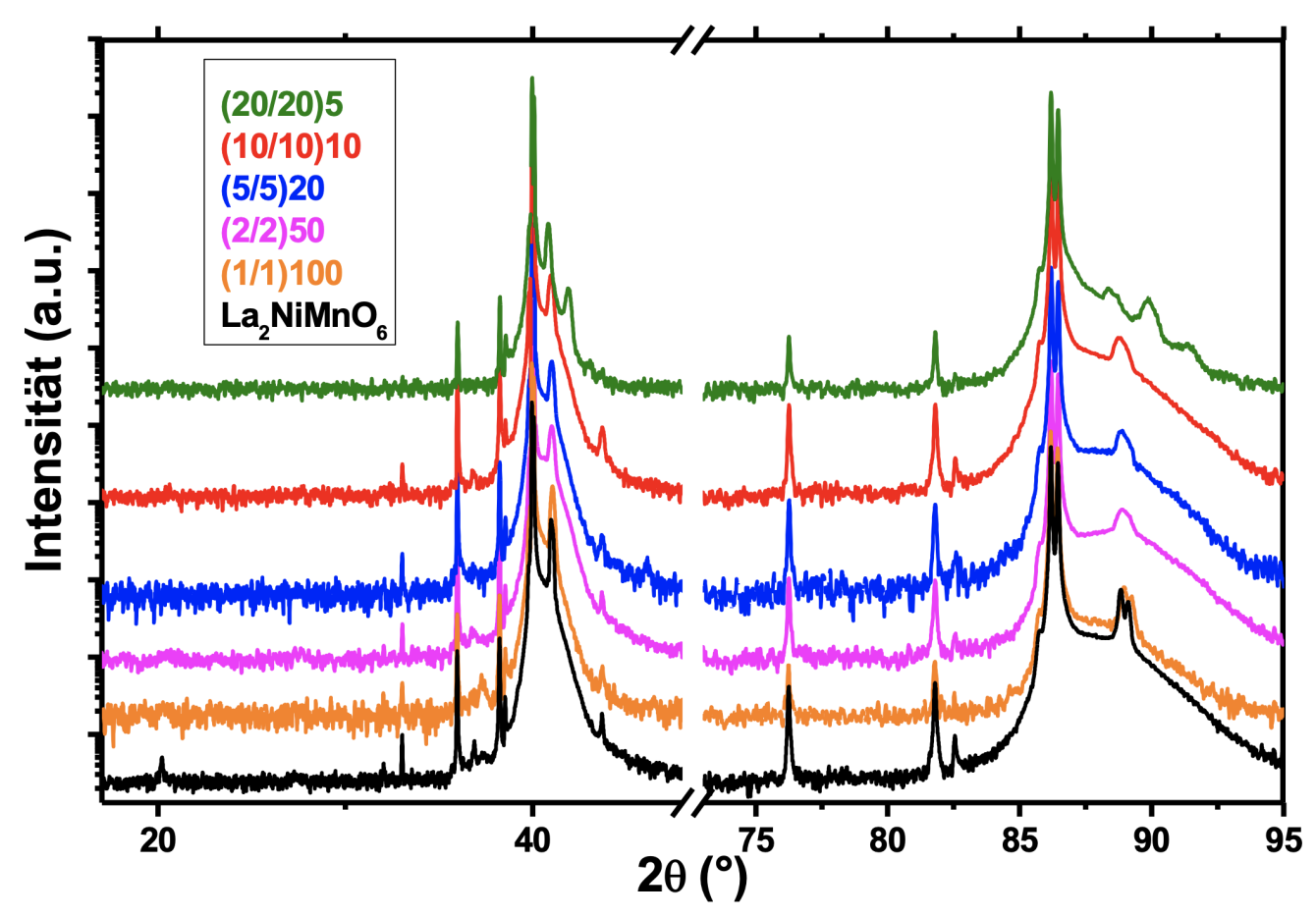

Abbildung A.3.: XRD-Messungen der Heterostrukturen $\left(\mathrm{LaMnO}_{3}\right)_{n} /$ $\left(\mathrm{LaNiO}_{3}\right)_{n}$ mit $n=20,10,5,2,1$ und der SoA-Probe. Mit Erlaubnis entnommen aus [155]. 


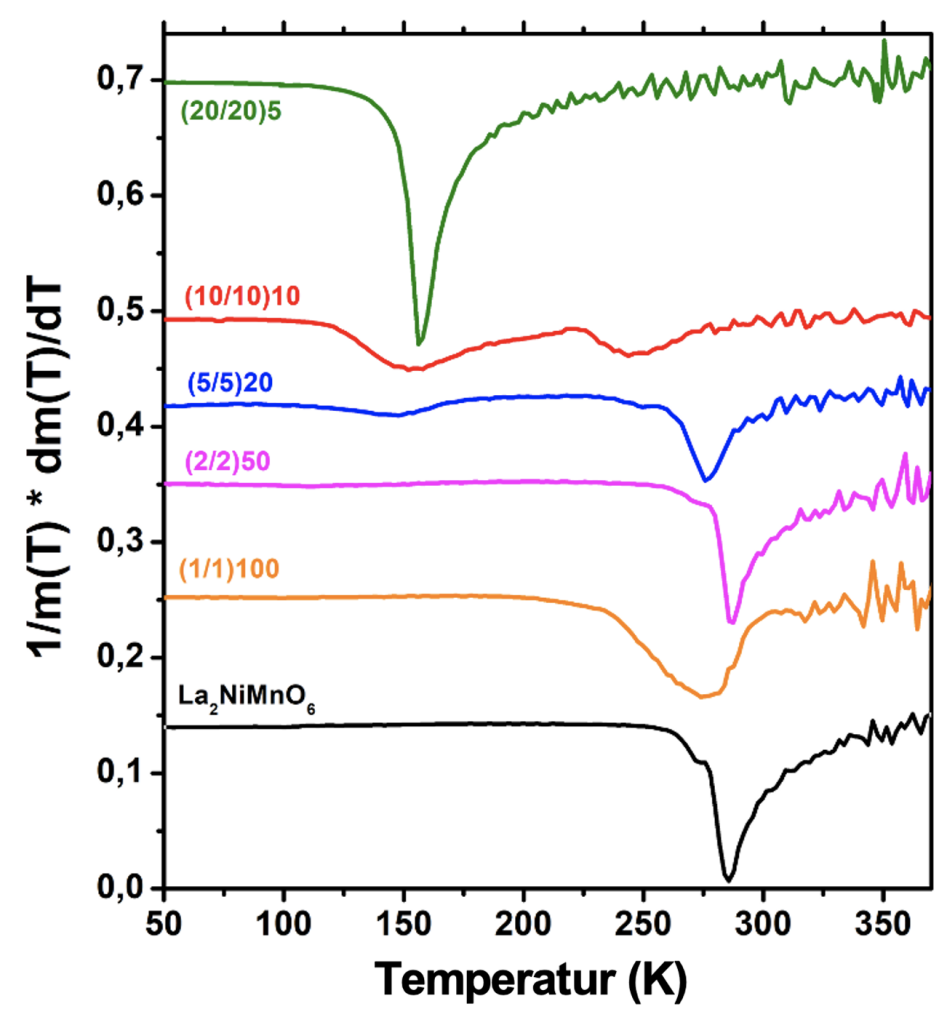

Abbildung A.4.: Logarithmische Ableitung der temperaturabhängigen Magnetisierungsmessungen der Heterostrukturen $\left(\mathrm{LaMnO}_{3}\right)_{n} /\left(\mathrm{LaNiO}_{3}\right)_{n}$ mit $n=20,10,5,2,1$ und der SoA-Probe. Mit Erlaubnis entnommen aus [155].

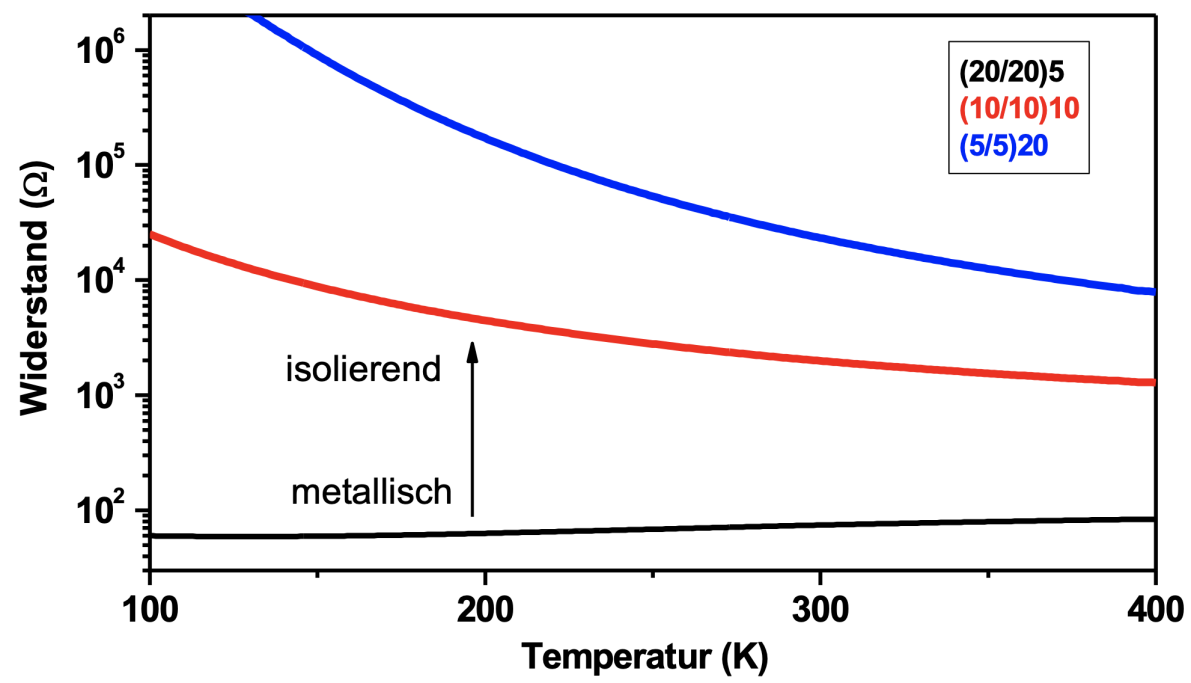

Abbildung A.5.: Elektrischer Widerstand der $\left(\mathrm{LaMnO}_{3}\right)_{n} /\left(\mathrm{LaNiO}_{3}\right)_{n}$ mit $n=20,10$ und 5. Mit Erlaubnis entnommen aus [155]. 


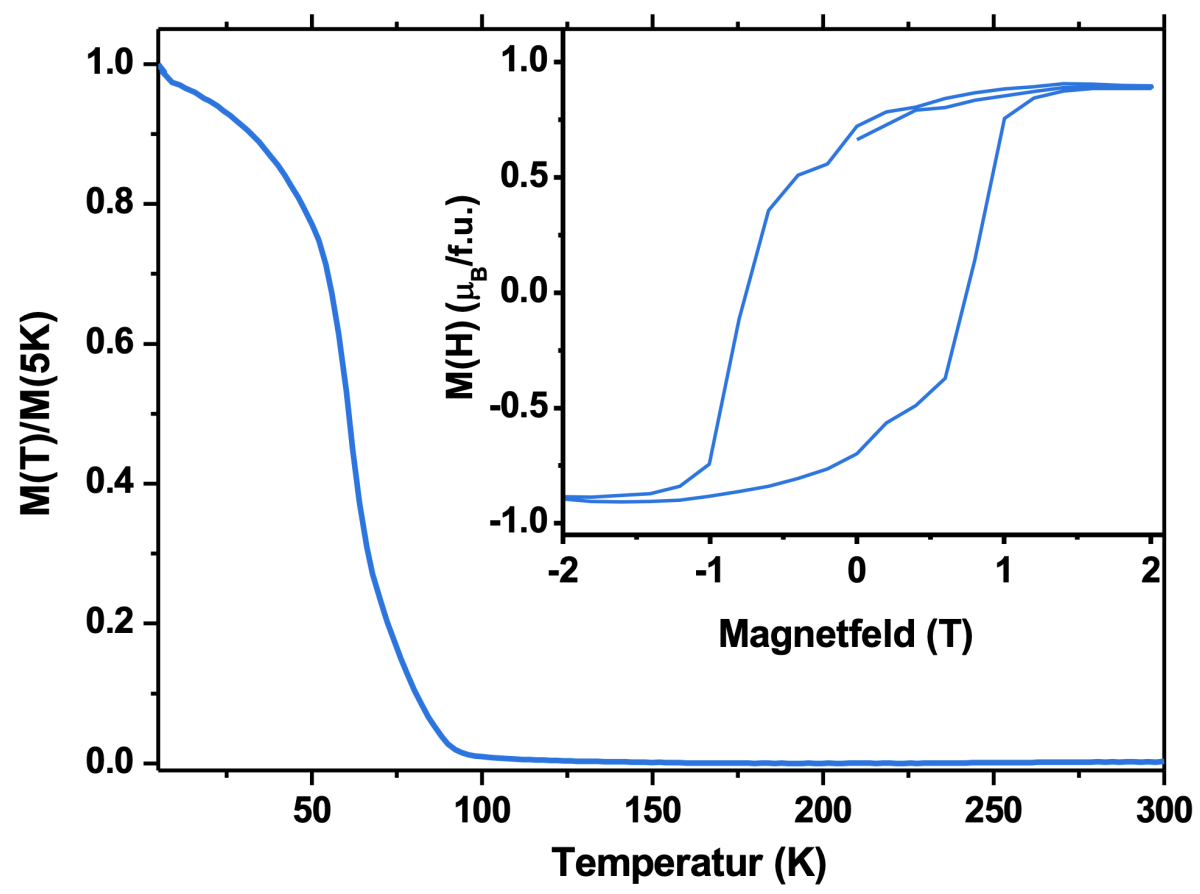

Abbildung A.6.: Magnetisierungsmessungen der $\mathrm{LaCoO}_{3}$-Probe auf $\mathrm{STO}(111) \mathrm{M}(\mathrm{H})$ bei $T=5 K$ und $\mathrm{M}(\mathrm{T})$ bei $H=1000 O e$. 


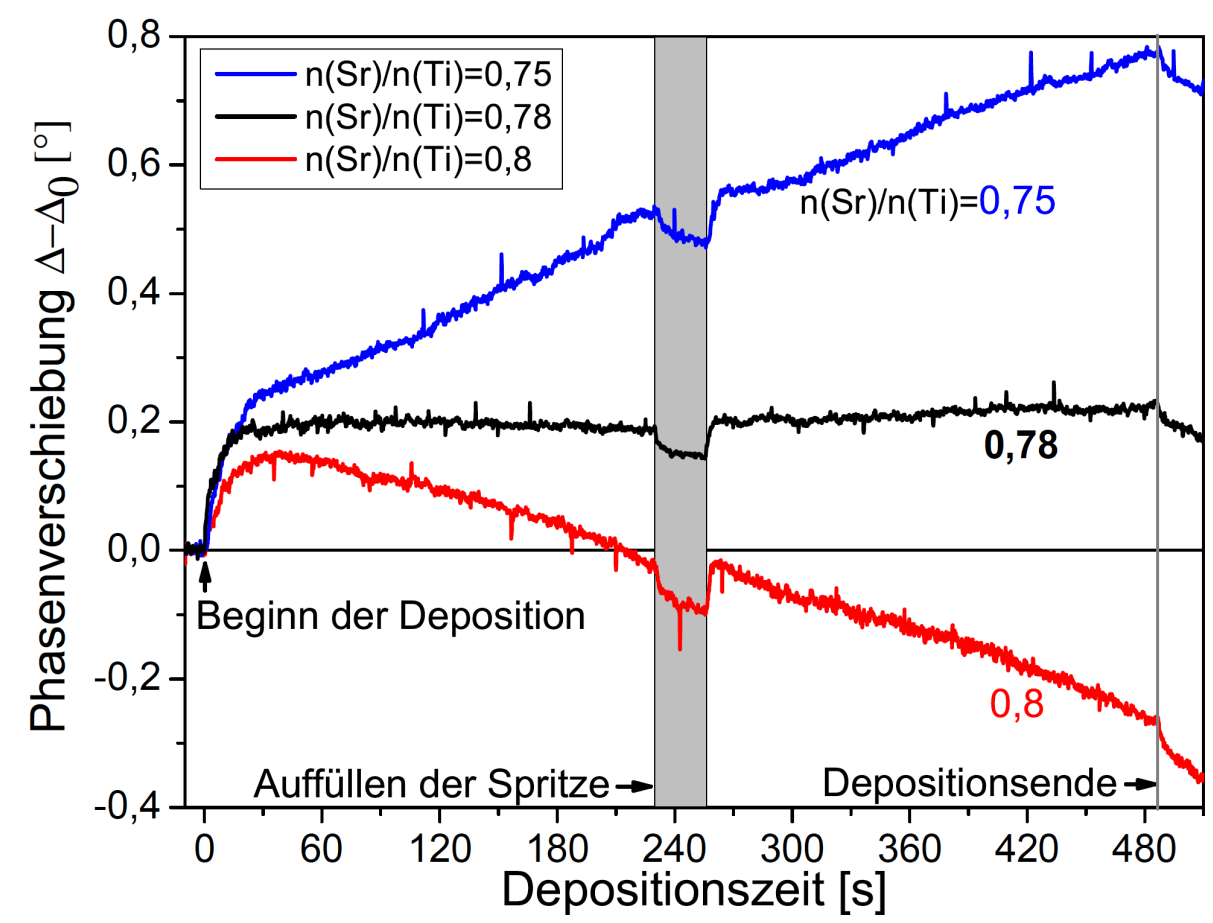

Abbildung A.7.: In-situ-Ellipsometriesignal der Phasenverschiebung $\Delta$ bei der Deposition von STO auf einem STO(100) Substrat in Abhängigkeit des Verhältnisses von $\mathrm{Ti} / \mathrm{Sr}$ in der Depositionslösung. Mit der Erlaubnis von Vitaly BruchmannBamberg entnommen aus der Masterarbeit von Vitaly Bruchmann-Bamberg [169].

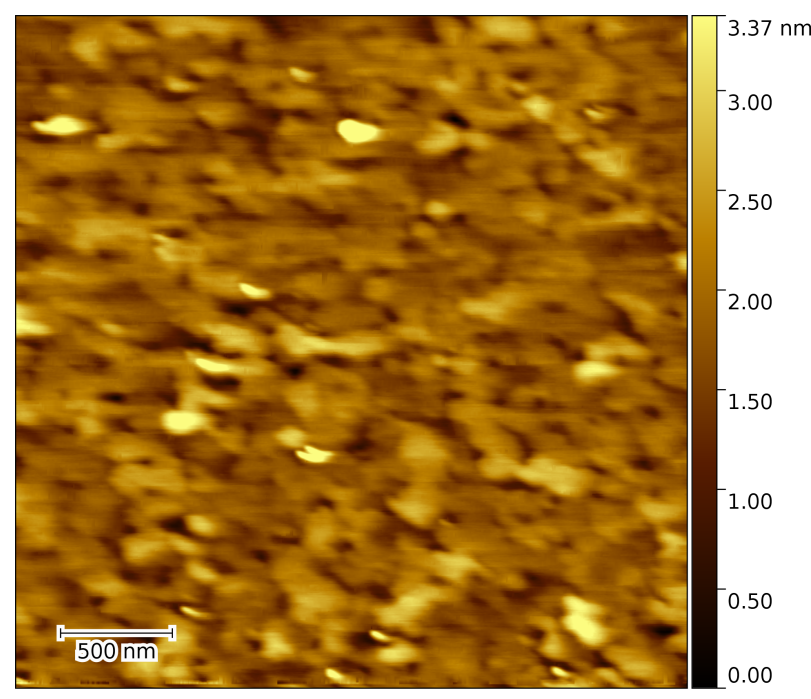

Abbildung A.8.: AFM-Aufnahme der $\mathrm{SrMnO}_{3}$-Einstellprobe auf STO(100) mit der Schichtdicke $d_{S M O}=5,1 \mathrm{~nm}$. 

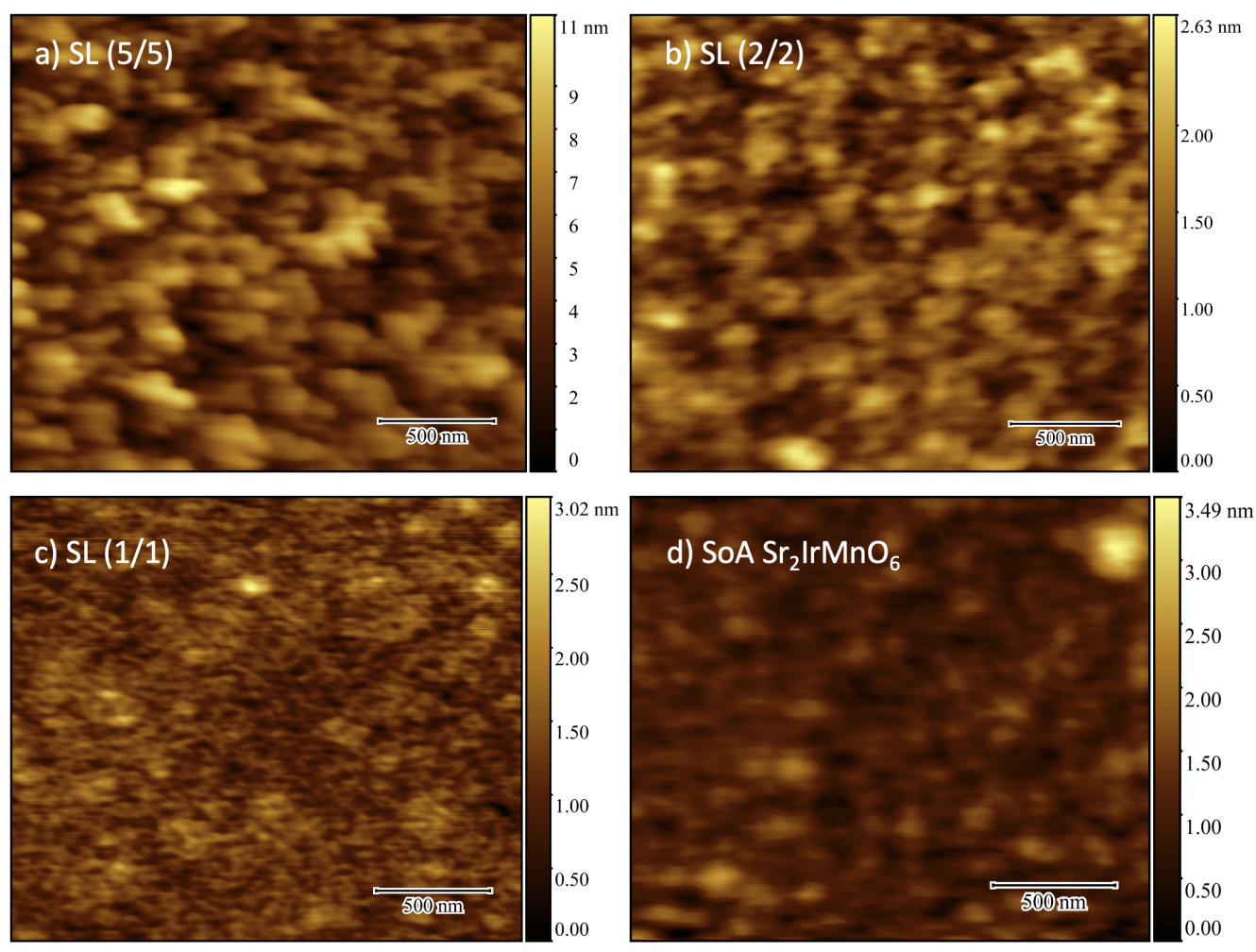

Abbildung A.9.: AFM-Aufnahmen der Oberflächenmorphologie der Heterostrukturen $\left(\mathrm{SrIrO}_{3}\right)_{n} /\left(\mathrm{SrMnO}_{3}\right)_{n}$ mit $n=5,2,1$ und der SoA-Probe auf STO(100).

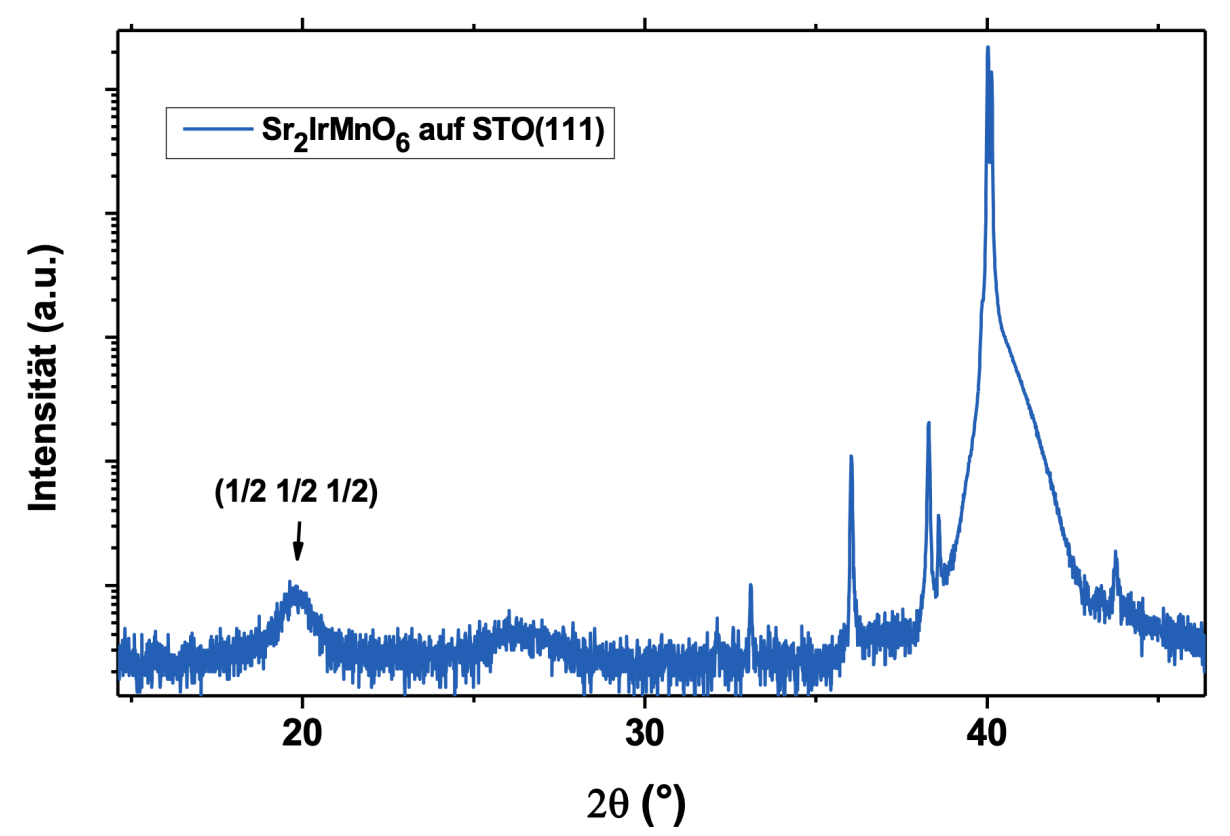

Abbildung A.10.: XRD-Messung der $\mathrm{Sr}_{2} \mathrm{IrMnO}_{6}$-Probe auf $\mathrm{STO}(111)$ mit einer Schichtdicke von $d_{S I M O}=44,9 \mathrm{~nm}$. 


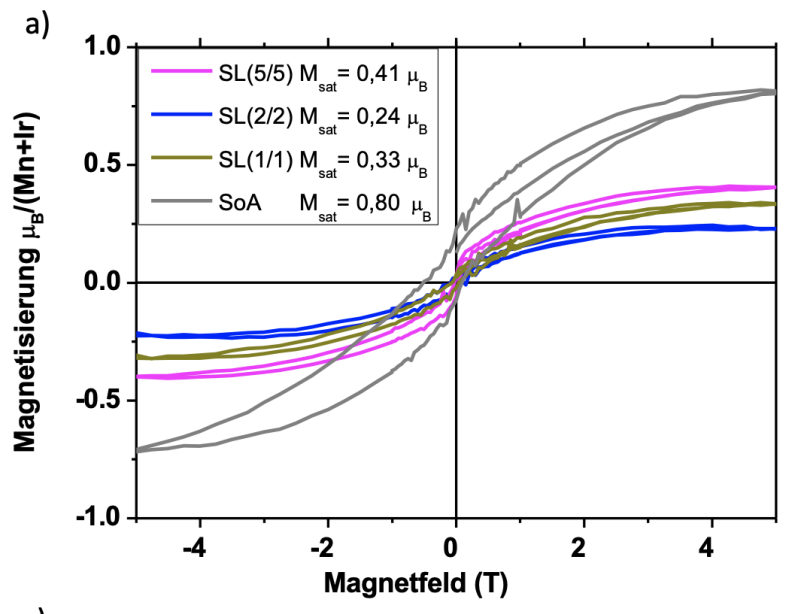

c)

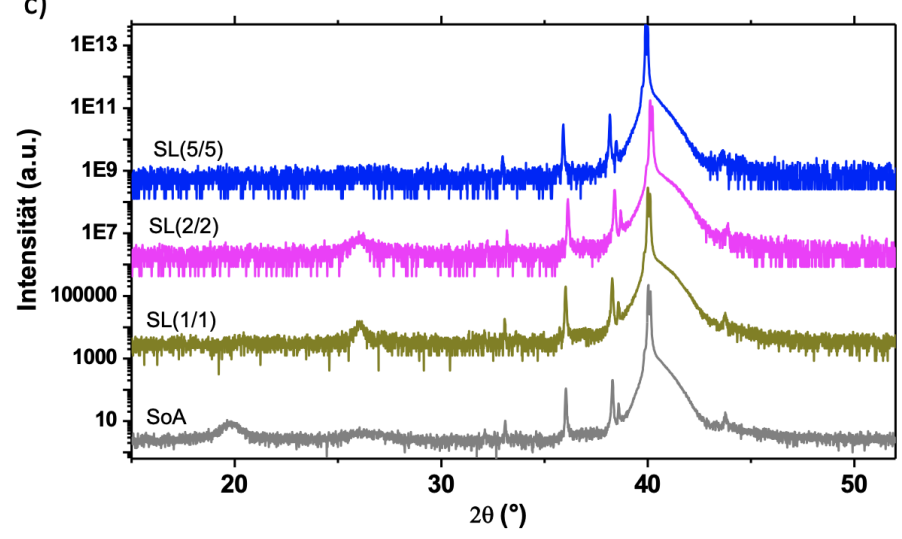

b)
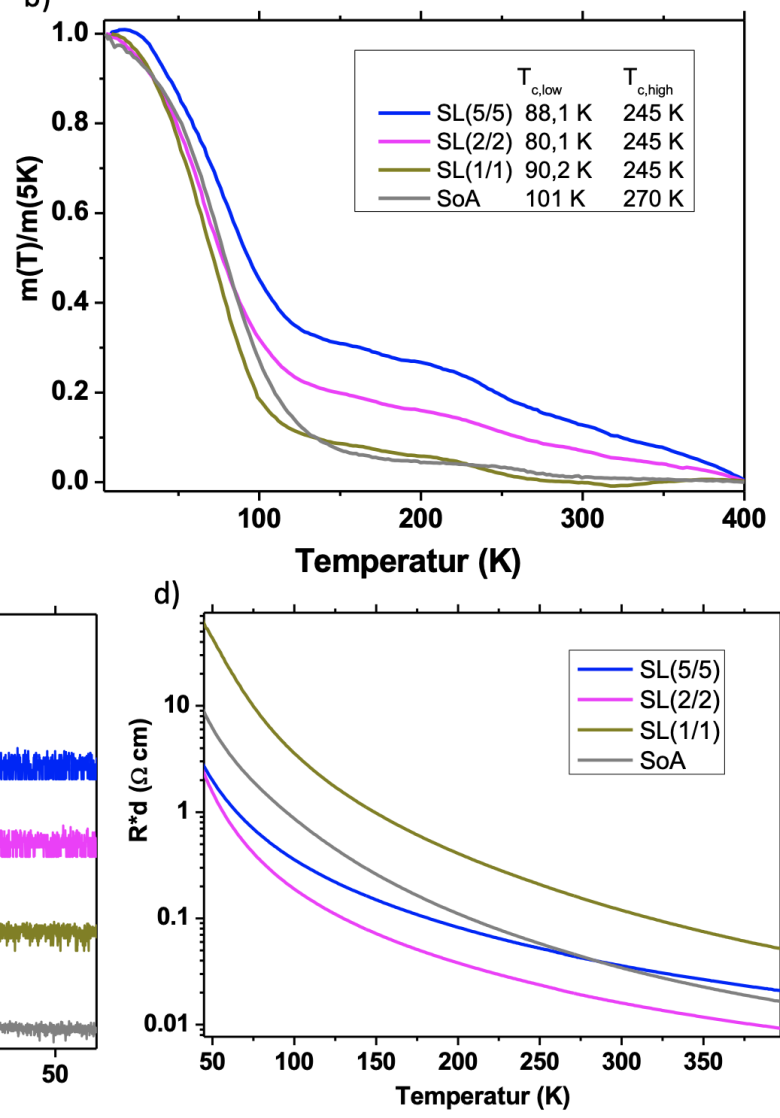

Abbildung A.11.: Untersuchungen an den Heterostrukturen $\left(\mathrm{SrIrO}_{3}\right)_{n} /$ $\left(\mathrm{SrIrO}_{3}\right)_{n}$ mit $\mathrm{n}=5,2$ und 1 und der SoA-Probe auf $\operatorname{STO}(111)$. a) Feldabhängige und b) temperaturabhängige Magnetisierungsmessung, c) XRD-Messung und d) Messung des spezifischen Flächenwiderstandes. 


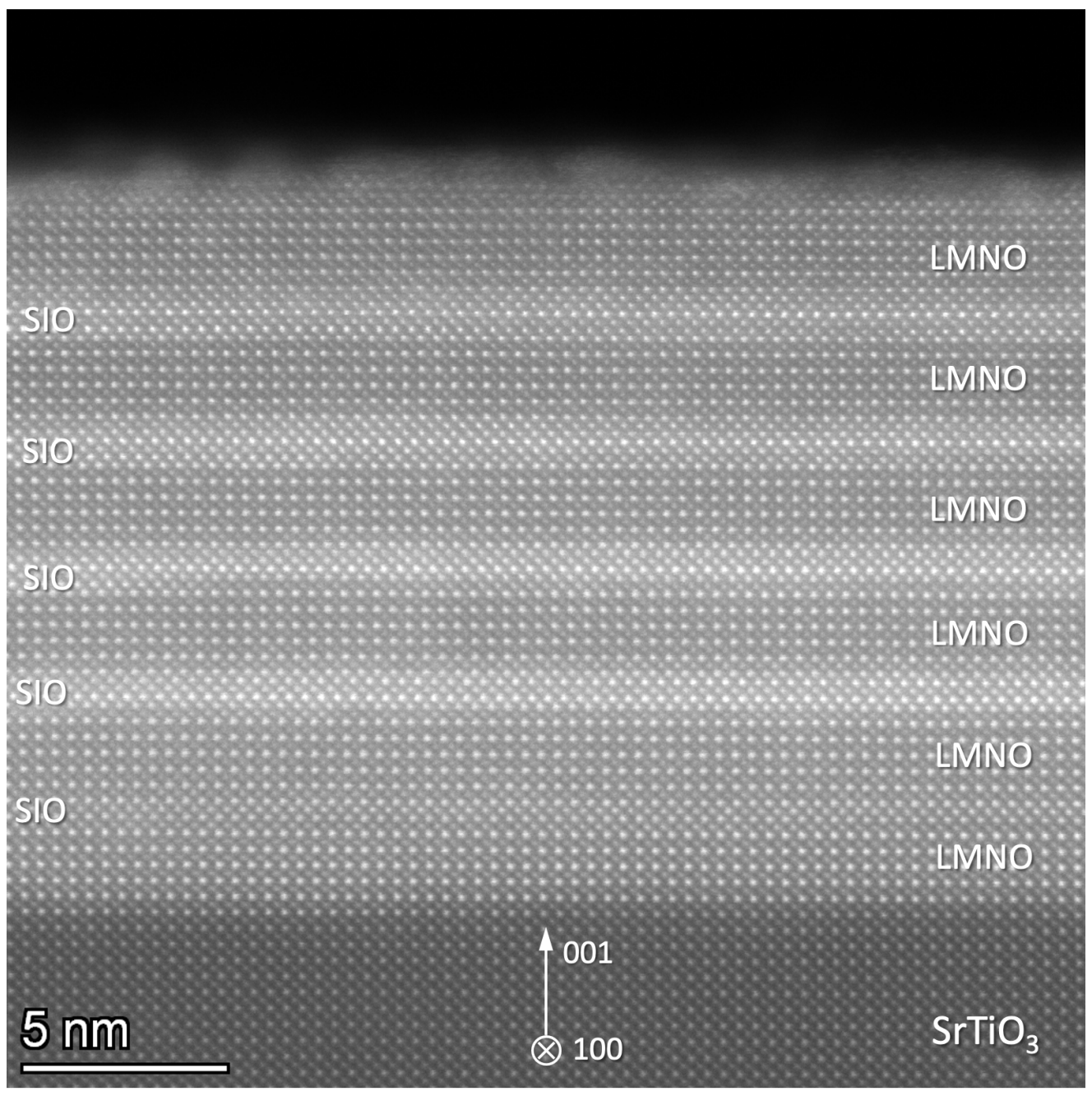

Abbildung A.12.: HAADF STEM-Aufnahme der $\quad\left(\left(\mathrm{La}_{2} \mathrm{NiMnO}_{6}\right)_{6} /\right.$ $\left.\left(\mathrm{SrIrO}_{3}\right)_{2}\right)_{6}$ Heterostruktur auf STO(100). 


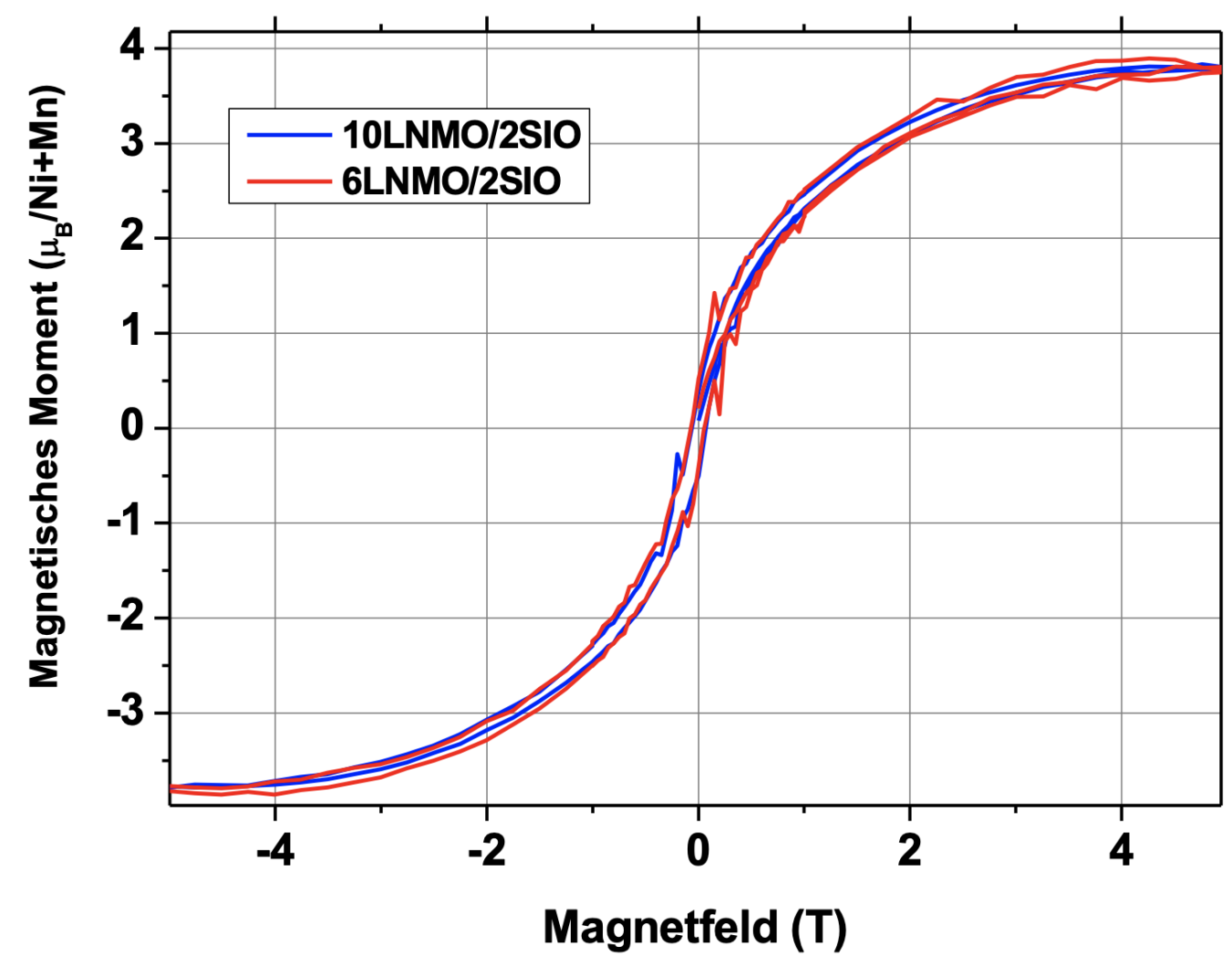

Abbildung A.13.: Feldabhängige Messungen der Magnetisierung der $\left(\left(\mathrm{La}_{2} \mathrm{NiMnO}_{6}\right)_{n} /\left(\mathrm{SrIrO}_{3}\right)_{2}\right)_{6}$ Heterostrukturen mit $\mathrm{n}=6$ und 10 bei $T=5 \mathrm{~K}$. Dabei wird davon ausgegangen, dass das gesamte Moment aus den $\mathrm{La}_{2} \mathrm{NiMnO}_{6}$ Einzellagen kommt. 


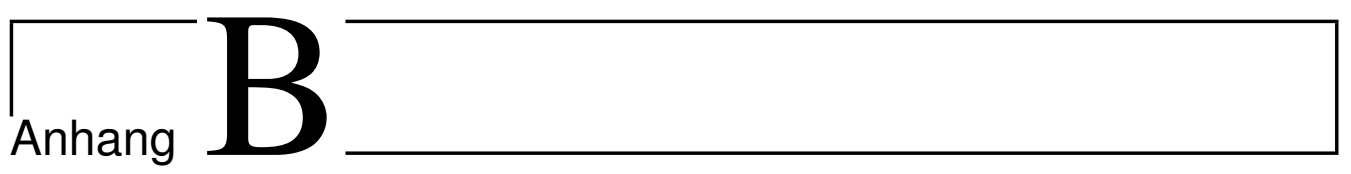

\section{Literaturverzeichnis}

1. G. H. Jonker, J. H. Van Santen, Ferromagnetic compounds of manganese with perovskite structure, Physica, Volume 16, 3, 337-349, 1950.

2. R. von Helmolt, J. Wecker, B. Holzapfel, L. Schultz, K. Samwer, Giant negative magnetoresistance in perovskitelike $\mathrm{La}_{2 / 3} \mathrm{Ba}_{1 / 3} \mathrm{MnO}_{x}$ ferromagnetic films, Phys. Rev. Lett., 71:2331-2333, 1993.

3. High $T_{c}$ Superconductors and Related Transition Metal Oxides, Springer Verlag, 2007.

4. J. G. Bednorz, K.A. Müller, Possible high $T_{c}$ superconductivity in the BaLaCuO system, Zeitschrift für Physik B Condensed Matter, 64(2):189193, 1986.

5. G. King, P. Woodwaerd, Cation ordering in Perovskites, J. Mater. Chem., 20, 5785-5796, 2010.

6. S. Vasala, M. Karppinen, $A_{2} B B^{\prime} O_{6}$ perovskites: $A$ review, Progress in Solid State Chemistry 43, 1-36, 2015.

7. D. D. Sarma, A new class of magnetic materials: $\mathrm{Sr}_{2} \mathrm{FeMoO}_{6}$ and related compounds, Curr. Opin. Solid State Mater. Sci., 5, 261-268, 2001.

8. B. P. Burton, E. Cockayne, Why $\mathrm{Pb}\left(B, B^{\prime}\right) O_{3}$ perovskites disorder at lower temperatures than $\mathrm{Ba}\left(B, B^{\prime}\right) \mathrm{O}_{3}$ perovskites, Phys. Rev. B: Condens. Matter Mater. Phys., 60, R12542-R12545, 1999. 
9. V. Moshnyaga, K. Samwer, Polaronic Emergent Phases in ManganiteBased Heterostructures, Crystals, 9, 489, 2019.

10. A. Bhattacharya, S. J. May, Magnetic Oxide Heterostructures, Annu. Rev. Matter. Res. 44: 65-90, 2014.

11. A. Ohtomo, H. Y. Hwang, A high-mobility electron gas at the $\mathrm{LaAlO}_{3} / \mathrm{SrTiO}_{3}$ heterointerface, Nature 427, 423, 2004.

12. M. Keunecke, F. Lyzwa, D. Schwarzbach, V. Roddatis, N. Gauquelin, K. Müller-Caspary, J. Verbeeck, S. J. Callori, F. Klose, M.Jungbauer, V. Moshnyaga, High-T Interfacial Ferromagnetism in $\mathrm{SrMnO}_{3} / \mathrm{LaMnO}_{3}$ Superlattices, Adv. Funct. Mater., 1808270, 2019.

13. B. J. Kim, H. Jin, S. J. Moon, J.-Y. Kim, B.-G. Park, C. S. Leem, J. Yu, T. W. Noh, C. Kim, S.-J. Oh, J.-H. Park, V. Durairaj, G. Cao, E. Rotenberg, Novel $J_{\text {eff }}=1 / 2$ Mott state induced by relativistic spin-orbit coupling in $\mathrm{Sr}_{2} \mathrm{IrO}_{4}$, Phys. Rev. Lett., 101:076402, 2008.

14. J. Matsouno et al., Interface-driven topological Hall effect in $\mathrm{SrRuO}{ }_{3}^{-}$ $\mathrm{SrIrO}_{3}$ bilayer, Sci. Adv. 2016;2:e1600304, 2016.

15. K. Y. Meng et al., Observation of Nanoscale Skyrmions in $\mathrm{SrRuO}_{3} / \mathrm{SrIrO}_{3}$ Bilayer, Nano Lett., 19, 3169-3175, 2019.

16. J. Nicholson et al., Emerging Magnetism and anomalous Hall effect in iridate-mnganite heterostructures, Nat. Commun., 7:12721, 2016.

17. S. Okamoto et al., Charge Transfer in Iridate-Manganite Superlattices, Nano. Lett., 17, 2126-2130, 2017.

18. S. Bhowal, S. Satpathy, Emerging Magnetism at the 3d-5d Interface: $\mathrm{SrMnO}_{3} / \mathrm{SrIrO}_{3}$, Adv. Mat. AIP Conf. Proc. 2005, 02000, 2018.

19. B. Göbel, I. Mertig, O. A. Tretiakov, Beyond skyrmions: Review and perspectives of alternative magnetic quasiparticles, Phys. Rep. 895, 1, 2021. 
20. E. Skoropata et al., Interfacial tuning of chiral magnetic interactions for large topological Hall effects in $\mathrm{LaMnO}_{3} / \mathrm{SrIrO}_{3}$ heterostructures, Sci. Adv. 2020, 6 : eaaz3902, 2020.

21. Y. Li et al., Emergent Topological Hall Effect in $\mathrm{La}_{0.7} \mathrm{Sr}_{0.3} \mathrm{MnO}_{3} / \mathrm{SrIrO}_{3}$ Heterostructures, ACS Appl. Mater. Interfaces, 11, 21268-21274, 2019.

22. K. Everschor-Sitte, J. Masell, R. M. Reeve, M. Kläui, Perspective: Magnetic skyrmions-Overview of recent progress in an active research field, J. Appl. Phys. 124, 240901, 2018.

23. D. J. Groenendijk, Spin-orbit Coupling and Gemetric Phases at Oxide Interfaces, Dissertation, Delft University of Technology, Niederlande, 2019.

24. A. Fert, N. Reyren, V. Cros, Magnetic skyrmions: advances in physics and potential applications, Nat. Rev. Mat. 2, 17031, 2017.

25. G. Finocchio et al., Magnetic skyrmions: from fundamental to applications, J. Phys. D: Appl. Phys., 49, 423001, 2016.

26. J. Inoue, H. Ohno, Taking the Hall Effect for a Spin, The American Association for the Advancement of Science, 2005.

27. H. Fujiwara, Spectroscopic Ellipsometry Principles and Applications, John Wiley and Sons, Ltd., The Atrium, Chichester, 2007.

28. K. Momma, F. Izumi, VESTA 3 for three-domensional visualization of crystal, volumetric and morphology data, J. Appl. Crystallogr., 44, 12721276, 2011.

29. V. M. Goldschmidt, Die Gesetze der Krystallochemie, Naturwissenschaften 21:477-85, 1926.

30. A. M. Glazer, Classification of tilted octahedra in perovskites, Acta Crystallogrsphica Seciton B 28:3384-92, 1972.

31. D. I. Khomskii, Transition Metal Compounds, Cambridge University Press, 1. Auflage, 2014. 
32. R. Gross, Achim Marx, Der Kolossale Magnetowiderstandseffekt, Kapitel 4 aus dem Vorlesungsskript: Spinelektronik, Walther-MeissnerInstitut, 2004.

33. Y. Tokura, Y. Tomiok, Colossal magnetoresitive manganites, J. Magn. Magn. Mater 200, 1, 1999.

34. H. A. Jahn, E. Teller, Stability of polyatomic molecules in degenerate electronic states. I. Orbital degeneracy, Proc. R. Soc. Lond. A 161, 220235, 1937.

35. D. Khomskii, Electronic structure, exchange and magnetism in oxides, in Spin Electronics, ed. M. J. Thornton, M. Ziese, 89-116, Deutschland, Berlin/Heidelberg, Springer-Verlag, 2001.

36. S. Middey, J. Chakhalin, P. Mahadevan, J. W. Freeland, A. J. Millis, D. D. Sarma, Physics of Ultrathin Films and Heterostructures of Rare-Earth Nickelates, Annual Review of Materials Research 46, 11.1-11.30, 2016.

37. D. Fuchs, E. Arac, C. Pinta, S. Schuppler, R. Schneider, H. v. Löhneysen, Tuning the magnetic properties of $\mathrm{LaCoO}_{3}$ thin films by epitaxial strain, Phys. Rev. B 77, 014434, 2008.

38. P. A. Dirac, R. H. Fowler, The Quantum Theory of the Elektron, Proceedings of the Royal Society of London A 117, 610, 1928.

39. H. Haken, H. C. Wolf, Atom- und Quantenphysik. Einführung in die experimentellen und theoretischen Grundlagen, 8. aktualisierte und erweiterte Auflage, Springer, Berlin, 2004.

40. B. Keimer, A. Aharony, A. Auerbach, R. J. Birgeneau, A. Cassanho, Y. Endoh, R. W. Erwin, M. A. Kastner, G. Shirane, Néel transition and sublattice magnetization of pure and doped $\mathrm{La}_{2} \mathrm{CuO}_{4}$, Phys. Rev. B 45, 7430, 1992.

41. B. Andlauer, J. Schneider, W. Tolksdorf, Optical Absorption, Fluorescence and Electron Spin Resonance of $\mathrm{Ir}^{+4}$ on Octahedral Sites in $Y_{3} \mathrm{Ga}_{5} \mathrm{O}_{12}$, Physica Status Solidi B 73, 533, 1976. 
42. G. L. Stamokostas, G A. Fiete, Mixing of $t_{2 g}-e_{g}$ orbitals in $4 d$ and $5 d$ transition metal oxides, Phys. Rev. B 97, 085150, 2018.

43. J. Hubbard, Electron correlations in narrow energy bands, Proceedings of the Royal Society of London A 276, 238, 1963.

44. J. Hubbard, Electron Correlations in Narrow Energy Bands. II. The Degenerate Band Case, Proceedings of the Royal Society of London A: Mathematical, Physical and Engineering Sciences, 277:237-259, 1964.

45. J. Hubbard, Electron Correlations in Narrow Energy Bands. III. An Improved Solution, Proceedings of the Royal Society of London A: Mathematical, Physical and Engineering Sciences, 281:401-419, 1964.

46. J. Hubbard, Electron Correlations in Narrow Energy Bands. IV. The Atomic Representation, Proceedings of the Royal Society of London A: Mathematical, Physical and Engineering Sciences, 285:542-560, 1965.

47. S. J. Moon et al, Dimensionality-Controlled Insulator-Metal Transition and Correlated Metallic State in 5d Transition Metal Oxides $S r_{n+1} I_{n} O_{3 n+1}(n=1,2$ and $\infty)$, Phys. Rev. Lett. 101, 226402, 2008.

48. N. Kovaleva et al., Low-energy Mott-Hubbard excitations in $\mathrm{LaMnO}_{3}$ probed by optical ellipsometry, Phys. Rev. B 81, 235130, 2010.

49. A. Gupta, T. R. McGuire, P. R. Duncombe, M. Rupp, J. Z. Sun, W. J. Gallagher, Gang Xiao, Growth And Giant Magnetoresistance Properties Of Ladeficient $\mathrm{La}_{x} \mathrm{MnO}_{3-\delta}(0,67<x<1)$, Appl. Phys. Lett. 67 (23), 4, 1995.

50. J. B. Goodenough, Theory of the role of covalence in the perovskite-type manganites [La,M(II)]MnO $\mathrm{M}_{3}$, Phys. Rev. 100:564-73, 1955.

51. J. Kanamori, Superexchange interaction and symmetry properties of electron orbitals. J. Phys. Chem. Solids 10:87-98, 1959.

52. P. W. Anderson, Antiferromagnetism. Theory of superexchange interaction, Phys. Rev. 79:350-56, 1950. 
53. D. I. Khomskii, G. A. Sawatzky, Interplay between spin, charge and orbital degrees of freedom in magnetic oxides, Solid State Communications, Vol. 102, No. 2-3, 87-99, 1997.

54. M. N. Iliev et al., Raman Spectroscopy of Ordered Double Perovskite $\mathrm{La}_{2} \mathrm{NiMnO}_{6}$ Thin Films, Phys. Rev. B, 75, 104118, 2007.

55. M. N. Iliev, H. Guo, A. Gupta, Raman Spectroscopy Evidence of Strong Spin-Phonon Coupling in Epitaxial Thin Films of the Double Perovskite $\mathrm{La}_{2} \mathrm{NiMnO}_{6}$, Appl. Phys. Lett., 2007, 90, 151914.

56. M. N. Iliev et al., Growth, magnetic properties, and Raman scattering of $\mathrm{La}_{2} \mathrm{NiMnO}_{6}$ single crystals, J. Appl. Phys., 106, 023515, 2009.

57. K. D. Truong et al., Fournier, P. Impact of Co/Mn cation ordering on phonon anomalies in $\mathrm{La}_{2} \mathrm{CoMnO}_{6}$ double perovsikter: Raman spectroscopy, Phys. Rev. B, 76, 132413, 2007.

58. K. D. Truong, M. P. Singh, S. Jandl, P. Fournier, Influence of Ni/Mn cation order on the spin-phonon coupling in multifunctional $\mathrm{La}_{2} \mathrm{NiMnO}_{6}$ epitaxial films by polarized Raman spectroscopy, Phys. Rev. B 80, 134424, 2009 .

59. C. Meyer, V. Roddatis, P. Ksoll, B. Damaschke and V. Moshnyaga, Structure, magnetism, and spin-phonon coupling in heteroepitaxial $\mathrm{La}_{2} \mathrm{CoMnO}_{6} / \mathrm{Al}_{2} \mathrm{O}_{3}$ (0001) films, Phys. Rev. B 98, 134433, 2018.

DIO: https://doi.org/10.1103/PhysRevB.98.134433 .

60. R. Takahashi et al., A-site driven ferroelectricity in strained ferromagnetic $\mathrm{La}_{2} \mathrm{NiMnO}_{6}$, Phys. Rev. B, 91, 134107, 2015.

61. J. J. P. Peters et al., Quantitative High-Dynamic-Range Electron Diffraction of Polar Nanodomains in $\mathrm{Pb}_{2} \mathrm{ScTaO}_{6}$, Adv. Mater., 31, 1806498, 2019.

62. C. Meyer, Ordnung-/Unordnungsphänomene in korrelierten Perowskitschichten anhand von fortgeschrittener Raman- Spektroskopie, Dissertation, Georg-August-Universität, Göttingen, 2018. 
63. P. Ksoll, C. Meyer, L. Schüler, V. Roddatis, V. Moshnyaga, B-site Cation Ordering in Films, Superlattices and Layer-by-Layer Grown Double Perovskites, Crystals 11, 734, 2021.

DOI: https://doi.org/10.3390/cryst11070734 .

64. R. D. Shannon, Revised effective ionic radii and systematic studies of interatomic distances in halides and chalcogenides, Acta Cryst. A32, 751$767,1976$.

65. S. R. Spurgeon et al., Competing Pathways for Nucleation of the Double Perovskite Structure in the Epitaxial Synthesis of $\mathrm{La}_{2} \mathrm{MnNiO}_{6}$, Chem. Mater., 28, 11, 3814-3822, 2016.

66. H. Z. Guo, A. Gupta, J. Zhang, M. Varela, S. J. Pennycook, Effect of oxygen concentration on the magnetic properties of $\mathrm{La}_{2} \mathrm{CoMnO}_{6}$ thin films, Applid Physics Letters 91, 202509, 2007.

67. J. E. Kleibeuker et al., Route to achieving perfect B-site ordering in double perovskite thin films, NPG Asia Materials 9, 406, 2017.

68. R. Galceran et al., Engineering the microstructure and magnetism of $\mathrm{La}_{2} \mathrm{CoMnO}_{3-\delta}$ thin films by tailoring oxygen stoichiometry, Appl. Phys. Lett. 105, 242401, 2014.

69. M. Berbal-Salamanca et al., Nonstoichiometry Driven Ferromagnetism in Double Perovskite $\mathrm{La}_{2} \mathrm{Ni}_{1-x} \mathrm{Mn}_{1+x} \mathrm{O}_{6}$ Insulating Thin Films, Cryst. Growth Des., 19, 5, 2765-2771, 2019.

70. M. Jungbauer, S. Hühn, R. Egoavil, H. Tan, J. Verbeeck, G. Van Tendeloo, V. Moshnyaga, Atomic layer epitaxy of Ruddlesden-Popper $\mathrm{SrO}\left(\mathrm{SrTiO}_{3}\right)_{n}$ films by means of metalorganic aerosol deposition, Appl. Phys. Lett. 105, 251603, 2014.

71. H. Wang et al., Spontaneous cationic ordering in chemical-solutiongrown $\mathrm{La}_{2} \mathrm{CoMnO}_{6}$ double perovskite thin films, NPG Asia Materials volume 11, article number: 44, 2019. 
72. V. Shabadi, M. Major, P. Komissinskiy, M. Vafaee, A. Radetinac, M. Baghaie Yazdi, W. Donner, L. Alff, Origin of superstructures in (double) perovskite thin films, J. Appl. Phys. 116, 114901, 2014.

73. R. Egoavil, S. Hühn, M. Jungbauer, N. Gauquelin, A. Béchéa, G. Van Tendeloo, J. Verbeecka, V. Moshnyaga, Phase problem in the B-site ordering of $\mathrm{La}_{2} \mathrm{CoMnO}_{6}$ - impact on structure and magnetism, Nanoscale, 7, 9835, 2015.

74. S. Chakraverty, A. Ohtomo, D. Okuyama, M. Saito, M. Okude, R. Kumai, T. Arima, Y. Tokura, S. Tsukimoto, Y. Ikuhara, M. Kawasaki, Ferrimagnetism and spontaneous ordering of transition metals in double perovskite $\mathrm{La}_{2} \mathrm{CrFeO}_{6}$ films, Phys. Rev. B 84, 064436, 2011.

75. N. S. Rogado, J. Li, A. W. Sleight, M. A. Subramanian, Magnetocapacitance and Magnetoresistence near Room Temperature in a ferromagnetic Semiconductor: $\mathrm{La}_{2} \mathrm{NiMnO}_{6}$, Adv. Mater. 17, 2225-2227, 2005.

76. M. P. Singh, K. D. Truong, S. Jandl, P. Fournier, Long-range Ni/Mn structural order in epitaxial double perovskite $\mathrm{La}_{2} \mathrm{NiMnO}_{6}$ thin films, Phys. Rev. B 79, 224421, 2009.

77. R. I. Dass, J.-Q. Yan, J. B. Goodenough, Oxigen stoichiometry, ferromagnetism, and transport properties of $\mathrm{La}_{2-x} \mathrm{NiMnO}_{6+\delta}$, Phys. Rev. B 68, 064415, 2003.

78. R. I. Dass, J. B. Goodenough, Multiple magnetic phase of $\mathrm{La}_{2} \mathrm{CoMnO}_{6-\delta}$ $(0 \leq \delta \leq 0.05)$, Phys. Rev. B 67, 014401, 2003.

79. R. J. Booth, R. Fillmann, H. Whitaker, A. Nag, R. M. Tiwari, K. V. Ramanujachary, J. Gopalakrishnan, S. E. Lofland, An investigation of structural, magnetic and dielectric properties of $R_{2} N_{i M N O}(R=$ rare earth, Y), Mat. Res. Bull. 44, 1559-1564, 2009.

80. C. Lin, S. Okamoto, A. J. Millis, Dynamical mean-field study of model double-exchange superlattices, Phys. Rev. B 73, 041104(R), 2006.

81. S. Okamoto, A. J. Millis, Electronic reconstruction at an interface between a Mott insulator and a band insulator, Nature 428, 630-633, 2004. 
82. M. Gibert, M. Viret, A. Torres-Pardo, C. Piamonteze, P. Zubko, N. Jaouen, J.-M. Tonnerre, A. Mougin, J. Fowlie, S. Catalano, A. Gloter, O. Stéphan, J.-M. Triscone, Interfacial Control of Magnetic Properties at $\mathrm{LaMnO}_{3} / \mathrm{LaNiO}_{3}$ Interfaces, Nano Lett. 15, 7355, 2015.

83. M. Gibert, M. Viret, P. Zubko, N. Jaouen, J.-M. Tonnerre, A. TorresPardo, S. Catalano, A. Gloter, O. Stephan, J.-M. Triscone, Interlayer coupling through a dimensionality-induced magnetic state, Nat. Commun. 7:11227, 2016.

84. M. Gibert, P. Zubko, R. Scherwitzl, J. Iniguez, J.-M. Triscone, Exchange bias in $\mathrm{LaNiO}_{3}-\mathrm{LaMnO}_{3}$ superlattices, Nat. Mater. 11, 195, 2012.

85. S. Dong, E. Dagotoo, Quantum confinement induced magnetism in $\mathrm{LaMnO}_{3} / \mathrm{LaNiO}_{3}$ superlattices, Phys. Rev. B 87, 195116, 2013.

86. J. Hoffman, I. C. Tung, B. B. Nelson-Cheeseman, M. Liu, J. W. Freeland, A. Bhattacharya, Charge transfer and interfacial magnetism in $\left(\mathrm{LaNiO}_{3}\right)_{3} /\left(\mathrm{LaMnO}_{3}\right)_{2}$ superlattices Phys. Rev. B 88, 144411, 2013.

87. C. Piamonteze, M. Gibert, J. Heidler, J. Dreiser, S. Rusponi, H. Brune, J.-M. Triscone, F. Nolting, U. Staub, Interfacial properties of $\mathrm{LaMnO}_{3} / \mathrm{LaNiO}_{3}$ superlattices grown along (001) and (111) orientations, Phys. Rev. B 92, 014426, 2015.

88. F. Lyzwa, P. Marsik, V. Roddatis, C. Bernhard, M. Jungbauer, V. Moshnyaga, In situ monitoring of atomic layer epitaxy via optical ellipsometry, J. Phys. D: Appl. Phys. 51 125306, 2018.

89. W. H. Meiklejohn, C. P. Bean, New Magnetic Anisotropy, Phys. Rev. $102,1413,1956$.

90. B. R. K. Nanda, S. Satpathy, Effects of strain on orbital ordering and magnetism at perovskite oxide interfaces: $\mathrm{LaMnO}_{3} / \mathrm{SrMnO}_{3}$ Phys. Rev. B 78, 054427, 2008.

91. C. Aruta, G. Ghiringhelli, A. Tebano, N. G. Boggio, N. B. Brookes, P. G. Medaglia, G. Balestrino, Strain induced x-ray absorption linear dichroism in $\mathrm{La}_{0.7} \mathrm{Sr}_{0.3} \mathrm{MnO}_{3}$ thin films, Phys. Rev. B 73, 235121, 2006. 
92. A. Tebano, C. Aruta, S. Sanna, P. G. Medaglia, G. Balestrino, A. A. Sidorenko, R. De Renzi, G. Ghiringhelli, L. Braicovich, V. Bisogni, N. B. Brookes, Evidence of Orbital Reconstruction at Interfaces in Ultrathin $\mathrm{La}_{0.67} \mathrm{Sr}_{0.33} \mathrm{MnO}_{3}$ Films, Phys. Rev. Lett. 100, 137401, 2008.

93. B. Cui, C. Song, F. Li, G. Y. Wang, H. J. Mao, J. J. Peng, F. Zeng, F. Pan, Tuning the entanglement between orbital reconstruction and charge transfer at a film surface, Sci. Rep. 4, 4206, 2014.

94. M. -B. Lepetit, B. Mercey, C. Simon, Interface Effects in Perovskite Thin Films, Phys. Rev. Lett. 108, 087202, 2012.

95. C. L. Jia et al., Oxygen octahedron reconstruction in the $\mathrm{SrTiO}_{3} / \mathrm{LaAlO}_{3}$ heterointerfaces investigated using aberration-corrected ultrahighresolution transmission electron microscopy, Phys. Rev. B 79:081405(R), 2009.

96. A. Y. Borisevich, H. J. Chang, M. Huijben, M. P. Oxley, S. Okamoto, M. K. Niranjan, J. D. Burton, E. Y. Tsymbal, Y. H. Chu, P. Yu, R. Ramesh, S. V. Kalinin, S. J. Pennycook, Suppression of Octahedral Tilts and Associated Changes in Electronic Properties at Epitaxial Oxide Heterostructure Interfaces, Phys. Rev. Lett. 105, 087204, 2010.

97. S. J. May, C. R. Smith, J.-W. Kim, E. Karapetrova, A. Bhattacharya, P. J. Ryan, Control of octahedral rotations in $\left(\mathrm{LaNiO}_{3}\right)_{n} /\left(\mathrm{SrMnO}_{3}\right)_{m}$ superlattices, Phys. Rev. B 83:153411, 2011.

98. J. M. Rondinelli, N. A. Spaldin, Substrate coherency driven octahedral rotations in perovskite oxide films, Phys. Rev. B 82:113402, 2010.

99. J. Topfer, J. B. Goodenough, $\mathrm{LaMnO}_{3+\delta}$ revisited, J. Solid State Chem. 130:117-28, 1997.

100. N. Nagaosa et al., Anomalous Hall effect, Rev. Mod. Phys., 82, 2, 2010.

101. R. Karplus, J. M. Luttinger, Hall Effect in Ferromagnetics, Phys. Rev. 95, 1154, 1954. 
102. J. M. Luttinger, Theory of the Hall Effect in Ferromagnetic Substances, Phys. Rev. 112, 739, 1958.

103. L. Berger, Side-Jump Mechanism for the Hall Effect of Ferromagnets, Phys. Rev. B 2, 4559, 1970.

104. L. Berger, Application of the Side-Jump Model to the Hall Effect and Nernst Effect in Ferromagnets, Phys. Rev. B 5, 1862, 1972.

105. M. V. Berry, Quantal phase factors accompanying adiabatic changes, Proc. R. Soc. London Ser. A 392, 45, 1984.

106. K. Ohgushi, S. Murakami, N. Nagaosa, Spin anisotropy and quantum Hall effect in the kagomé lattice: Chiral spin state based on a ferromagnet, Phys. Rev. B 62, 2000.

107. R. Gross, A. Marx, Festkörperphysik, Oldenbourg Verlag, 1. Auflage, 2012.

108. I. Dzyaloshinsky, A thermodynamic theory of weak ferromagnetism of antiferromagnetics, J. Phys. Chem. Solids 4, 241?255, 1958.

109. T. Moriya, Anisotropic superexchange interaction and weak ferromagnetism, Phys. Rev. 120, 91, 1960.

110. M. N. Grisolia et al., Hybridization-controlled charge transfer and induced magnetism at correlated oxide interfaces, Nat. Phys. 2016, 12, 484.

111. T. Shimizu, T. Yamaguchi, Y. Nishikawa, Semiconductor device and method for manufacturing same, U.S. Patent US 20050139926, 2005.

112. S. Okamoto, Magnetic interaction at an interface between manganite and other transition metal oxides, Phys. Rev. B 82, 024427, 2010.

113. V. Moshnyaga, I. Khoroshun, A.Sidorenko, P. Petrenko, A. Weidinger, M. Zeitler, B. Rauschenbach, R. Tidecks, K.Samwer, Preperation of rare-earth manganiteoxide thin films by metalorganic aerosoldeposition technique, App. Phys. Lett. 74, 2842, 1999. 
114. T. Nishinaga, H. J. Scheel, Crystal growth aspect of high-Tc superconductors In Advances in Superconductivity VIII, Hayakawa, H., Enomoto, Y., Eds., Springer: Tokyo, Japan, 33-38, 1996.

115. J. J. De Yoreo, P. G. Vekilov, Principles of crystal nucleation and growth, Rev. Mineral. Geochem., 54, 57-93, 2003.

116. S. Hühn, Kationen-Ordnung in ferri/ferromagnetischen perowskitischen Duennfilmen, Dissertation, Universität Göttingen, 2015.

117. T. Manako, M. Izumi, Y. Konishi, K. Kobayashi, Epitaxial thin films of ordered double perovskite $\mathrm{Sr}_{2} \mathrm{FeMoO}_{6}$, Appl. Phys. Lett. Vol. 74, Nr. 15, 1999.

118. U. Schwingenschlögel, V. Eyert, The vanadium Magnéli phases $V_{n} O_{2 n-1}$, In: arXiv preprint cond-mat/0403689, 2004.

119. S. Nakhal, Synthese, Kristallstruktur und Eigenschaften von neuen Übergangsmetalloxiden, -oxidhydroxiden und - Oxidnitriden, Dissertation, Technische Universität Berlin, 2008.

120. S. Esser, Untersuchung der Elektronen-Gitter Korrelation in $\mathrm{VO}_{2}$ Dünnfilmen, Masterarbeit, Universität Göttingen, 2016.

121. HiTec Zang GmbH, Ebertstraße 28-32 52134 Herzogenrath, Deutschland.

122. M. Bass, Optical Society of America. Handbook of Optics, 3td edition, McGraw-Hill Companies, 2010.

123. S. N. Jasperson, S. E. Schnatterly, An Improved Method for High Reflectivity Ellipsometry Based on a New Polarization Modulation Technique, Review of Scientific Instruments 40, 761, 1969.

124. M. Jungbauer, Gestaltung der elektronischen Korrelation in PerowskitHeterostrukturen auf atomarer Skala, Dissertation an der Universität Göttingen, 2015.

125. Hinds-Instruments Inc., 7245 NE Evergreen Pkwy, Hillsboro, PEM-90 Photoelastic Modulator Systems User Manual, 1994. 
126. Keithley Instruments, Model 2000 Multimeter Users Manual, 1994.

127. Stanford Research Systems,1290-D Reamwood Ave., Sunnyvale, CA 94089, SR830 Lock-In Amplifier Manual, 2011.

128. Bruker Corporation, 40 Manning Road, Billerica, MA 01821, United Sates, Innova - Superior AFM Research Performance and Versatility, Broschüre, 2016.

129. G. Binnig, C.F.Quate, Atomic Force Microscope. Phys. Rev. Lett. 56, 9, 930-933, 1986.

130. P. Klapetek, D. Necas, C. Anderson, Gwyddion user guide, Anleitung, 2016.

131. V. Kanniah, E. A. Grulke, T. Druffel, The effects of surface roughness on low haze ultrathin nanocomposite films, Thin Solid Films 539, 170-180, 2013.

132. B. Fultz, J. M. Howe, Transmission Electron Microscopy and Diffractometry of Materials, Springer Verlag, 3. Auflage, 2008.

133. R. F. Egerton, Physical Principles of Electron Microscopy, Springer Verlag, 1. Auflage, 2005.

134. L. Reimer, H. Kohl, Transmission Electron Microscopy, Springer Verlag, 5. Auflage, 2008.

135. N. W. Ashcroft, D. N. Mermin, Festkörperphysik, Oldenbourg Verlag, 3. Auflage, 2007.

136. S. Hunklinger, Festkörperphysik, Oldenbourg Verlag, 2. Auflage, 2009.

137. B. D. Cullity, S. R. Stock, Elements of X-Ray Diffraction, Pearson Education, Addison-Wesley Publishing Company, 3. Auflage, 1956.

138. K. Kopitzki, P. Herzog, Einführung in die Festkörperphysik, Vieweg+Teubner Verlag, 6. Auflage, 2009.

139. M. Birkholz, Thin Film Analysis by X-Ray Scattering, Wiley-VCH, 2006. 
140. P. Debye, Interferenz von Röntgenstrahlen und Wärmebewegung, Ann. d. Phys. 348, 49-92, 1913.

141. I. Waller, Zur Frage der Einwirkung der Wärmebewegung auf die Interferenz von Röntgenstrahlen, Zeitschrift für Physik 17, 398-408, 1923.

142. L. G. Parratt, Surface Studies of Solids by Total Reflection of X-Rays, Phys. Rev. 95, 359-369, 1954.

143. M. Björck, G. Andersson, GenX: An Extensible X-Ray Reflectivity Refinement Program Utilizing Differential Evolution, J. Appl. Cryst. 40, 1174-1178, 2007.

144. C. V. Raman, A new radiation, Indian J. Phys. 2, 387-398, 1928.

145. C. V. Raman, K. S. Krishnan, A New Type of Secondary Radiation, Nature 121, 501, 1928.

146. C. V. Raman, The Molecular Scattering of Light, Nobel Lecture, 1930.

147. E. Smith, G. Dent, Modern Raman Spectroscopy - A Practical Approach, John Wiley and Sons Verlag, 1. Auflage, 2005.

148. M. Nicklaus, Tip-Enhanced Raman Spectroscopy For Nanoelectronics, Books on Demand Verlag, 1. Auflage, 2014.

149. Horiba Scientific, LabRAM HR Evolution User Manual, 2013.

150. J. Clarke, A. I. Braginski, The SQUID Handbook: Fundamentals and Technology of SQUIDs and SQUID Systems, Wiley, 2006.

151. K. Iwansson, G. Sinapius, W. Hoornaert, S. Middelhoek, Measuring Current, Voltage and Power. Handbook of Sensors and Actuators. Elsevier Science, 1999.

152. Quantum Design, PPMS Hardware Manual, 2004.

153. L. Scheuchenpflug, Untersuchung von $\mathrm{SrMnO}_{3} / \mathrm{SrIrO}_{3}$-DünnfilmeHeterostrukturen mittels Hall- und Magnetowiderstand, Bachelorarbeit, Universität Augsburg, Lehrstuhl für Experimentalphysik VI, 2021. 
154. F. Pérez-Willard, High Throughput Imaging with ZEISS Crossbeam 550, Carl Zeiss Microscopy GmbH, Oberkochen, Germany, 2016.

155. P. Ksoll, R. Mandal, C. Meyer, L. Schüler, V. Roddatis, V. Moshnyaga, Emergent Double Perovskite Phase at LaMnO $\mathrm{O}_{3} / \mathrm{LNiO}_{3}$ Interfaces: Coupled Charge Transfer and Structural Reconstruction, Phys. Rev. B 103, 195120, 2021.

DOI: https://doi.org/10.1103/PhysRevB.103.195120

156. Crystal GmbH, Geschäftsführer: Andreas Wenzel, Ostendstraße 25, 12459 Berlin, https://crystal-gmbh.com/

157. E. O. Wollan, W. C. Koehler, Neutron Diffraction Study of the Magnetic Properties of the Series of Perovskite-Type Compounds /(1x)La,xCa]MnO $\mathrm{M}_{3}$, Phys. Rev. 100, 545, 1955.

158. J. Roqueta, A. Pomar, L. Balcells, C. Frontera, S. Valencia, R. Abrudan, B. Bozzo, Z. Konstantinovic, J. Santiso, B. Martinez, StrainEngineered Ferromagnetism in $\mathrm{LaMnO}_{3}$ Thin Films, Cryst. Growth Des. 15, 5332-5337, 2015.

159. A. Chaturvedi, V. Sathe, Thickness Dependent Raman Study of Epitaxial LaMnO $\mathrm{O}_{3}$ Thin Films, Thin Solid Films 548, 75-80, 2013.

160. V. G. Dubey, R. Sathe, J. Rawat, Signature of Jahn-Teller Distortion and Oxygen Stoichiometry in Raman Spectra of Epitaxial $\mathrm{LaMnO}_{3+\delta}$ Thin Films, Appl. Phys. 104, 113530, 2008.

161. K. P. Rajeev, G. V. Shivashankar, A. K. Raychaudhuri, LowTemperature Electronic Properties of a Normal Conducting Perovskite Oxide ( $\mathrm{LaNiO}_{3}$ ), Solid State Commun. 79, 591-595, 1991.

162. J. L. García-Munoz, J. Rodríguez-Carvajal, P. Lacorre, J. B. Torrance, Neutron-Diffraction Study of $\mathrm{RNiO}_{3}(\mathrm{R}=\mathrm{La}, \mathrm{Pr}, \mathrm{Nd}, \mathrm{Sm})$ : Electronically Induced Structural Changes Across the Metal-Insulator Transition, Phys. Rev. B 46, 4414, 1992. 
163. K. M. Satyalakshmi, R.M. Mallya, K.V. Ramanathan, X. D. Wu, B. Brainard, D. C. Gautier, N. Y. Vasanthacharya, M. S. Hegde, Epitaxial Metallic LaNiO 3 Thin Films Grown by Pulsed Laser Deposition, Appl. Phys. Lett. 62, 1233-1235, 1993.

164. N. Chaban, M. Weber, S. Pignard, J. Kreisel, Phonon Raman Scattering of Perovskite $\mathrm{LaNiO}_{3}$ Thin Films, Appl. Phys. Lett. 97, 031915, 2010 .

165. G. Gou, I. Grinberg, A. Rappe, J. Rondinelli, Lattice Normal Modes and Electronic Properties of the Correlated Metal LaNiO ${ }_{3}$, Phys. Rev. B 84, 144101, 2011.

166. J. H. Gruenewald, J. Nichols, S.S.A. Seo, Pulsed Laser Deposition with Simultaneous In Situ Real-Time Monitoring of Optical Spectroscopic Ellipsometry and Reflection High-Energy Electron Diffraction, Rev. Sci. Instrum. 84, 043902, 2013.

167. Z. G. Hu, Z. M. Huang, Y. N. Wu, Q. Zhao, G. S. Wang, J. H. Chu, Ellipsometric Characterization of $\mathrm{LaNiO}_{3-x}$ Films Grown on Si(111) Substrates: Effects of Oxygen Partial Pressure, J. Appl. Phys. 95, 4036-4041, 2004 .

168. P. Ksoll, Wachstum von oxidischen Mikrostrukturen mittels Metallorganischer Aerosol Deposition, Masterarbeit, Universität Göttingen, 2017.

169. V. Bruchmann-Bamberg, Magnetische (Ent-) Kopplungen in Perowskit-Heterostrukturen, Masterarbeit, Universität Göttingen, 2017.

170. L. Schüler, Präperation von Doppelperowskitschichten mittels MADTechnik, Bachelorarbeit, Universität Göttingen, 2018.

171. K. Nogami, K. Yoshimatsu, H. Mashiko, E. Sakai, H. Kumigashira, O. Sakata, T. Oshima, A. Ohtomo, Epitaxial Synthesis and Electronic Properties of Double-Perovskite $\mathrm{Sr}_{2} \mathrm{TiRuO}_{2}$ Films, Applied Physics Express 6, 105502, 2013. 
172. Y. Cao, X. Liu, M. Kareev, D. Choudhury, S. Middey, D. Meyers, J.-W. Kim, P. J. Ryan, J. W. Freeland, J. Chakhalian, Engineered Mott ground state in a $\mathrm{LaTiO}_{3+\delta} / \mathrm{LaNiO}_{3}$ heterostructure, Nat. Comm., 7:10418, 2015.

173. J. Zhang et al., Antiferromagnetic interlayer coupling and thus induced distinct spin texture for the $\left[\mathrm{LaMnO}_{3} / \mathrm{LaCoO}_{3}\right]_{3}$ superlattices, Nanoscale, 9, 3476-3484, 2017.

174. L. Wu et al., Ferromagnetism and matrix-dependent charge transfer in strained $\mathrm{LaMnO}_{3}-\mathrm{LaCoO}_{3}$ superlattices, Mater. Res. Lett., 6 (9), 501507, 2018.

175. X. Guan et al., Tuning ganetism and crystal orientations by octahedral coupling in $\mathrm{LaCoO}_{3} / \mathrm{LaMnO}_{3}$ thin film, Phys. Rev. B, 100, 014427, 2019.

176. J. Chang et al., Emergence of Room-Temperature Magnetic Ordering

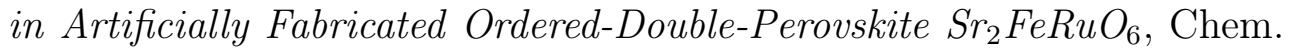
Mater., 23, 2693-2696, 2011.

177. A. Bhattacharya, S. J. May, S. G. E. te Velthuis, M. Warusawithana, X. Zhai, Bin Jiang, J.-M. Zuo, M. R. Fitzsimmons, S. D. Bader, J. N. Eckstein, Metal-Insulator Transition and Its Relation to Magnetic Structure in $\left(\mathrm{LaMnO}_{3}\right)_{2 n} /\left(\mathrm{SrMnO}_{3}\right)_{n}$ Superlattices, Phys. Rev. Lett., 100:257203, 2008.

178. T. Negas, R. S. Roth, The system $\mathrm{SrMnO}_{3-x}$, J. of Sol. State Chem., 1(3):409 - 418, 1970.

179. M. Keunecke, Grenzflächenmagnetismus in Manganat Übergittern, Masterarbeit, Universität Göttingen, 2016.

180. D. L. Smith, Thin-film deposition: principles and practice, Mcgraw Hill/Irwin Professional, 1995.

181. J. A. Venables, G. D. T. Spiller, M. Hanbucken, Nucleation and growth of thin films, Rep. Prog. Phys., 47 (4):399, 1984. 
182. P. K. Davies, H. Wu, A. Y. Borisevich, I. E. Molodetsky, L. Farber, Crystal Chemistry of Complex Perovskites: New Cation-Ordered Dielectric Oxides, Annu. Rev. Mater. Res. 38:369-401, 2008.

183. L. Bergmann et al., Growth-sequence-dependent interface magnetism of $\mathrm{SrIrO} \mathrm{O}_{3} / \mathrm{La}_{0.7} \mathrm{Sr}_{0.3} \mathrm{MnO}_{3}$ bilayers, AIP Advances 10, 035132, 2020. 


\section{Danksagung}

Vasily sagt immer, dass die Danksagung das Erste ist, das die Leute lesen werden. Man könnte also glatt meinen, dass es der bedeutungsvollste Teil der Arbeit sei. Und da ist auch was dran. Auf dem Deckblatt steht zwar mein Name, doch den weiten Weg bis hier her hätte ich alleine nicht geschafft. So ist diese Arbeit irgendwo auch auch ein Gesamtwerk vieler Leute, denen ich in tiefer Dankbarkeit verbunden bin.

Auch wenn man sagt, dass das Beste zum Schluss kommt, fange auch ich traditionell mit meinem Doktorvater, Erstgutachter und Freund Prof. Dr. Vasily Moshnyaga an. Das waren abwechslungsreiche, gemeinsame Jahre, die hinter uns liegen und in denen zweifelsfrei ein ganz besonderes Verhältnis entstanden ist. Ich bin dir für deine gesamte Unterstützung dankbar, ohne die ich Arbeit so nicht fertig bekommen hätte. Dabei ist es bis heute beeindruckend zu sehen, wie dein wissenschaftliches Feuer scheinbar nie zu erlöschen scheint. Diesen unermüdlichen Tatendrang und Wissbegier habe ich in der Form noch nie bei jemanden beobachten können. Darum hoffe ich auch weiterhin, dass dieses Feuer vielen kommenden Lehrenden zu Teil wird. Denn dieses Feuer ist in Verbindung mit deiner besonderen, liebevollen Art eine ganz außergewöhnliche Erfahrung, die mich für immer geprägt haben wird. Für all das danke ich dir und werde die gemeinsame Zeit nie vergessen.

Mein Dank gilt Prof. Dr. Christian Jooß für ihre Bereitschaft meine Arbeit als Korreferenten zu begutachten und sich den gesamten Aufwand dahinter anzunehmen. Mein Dank richtet sich auch an Prof. Dr. Michael Seibt, Prof. Dr. Stefan Mathias, Prof. Dr. Wolfram Kollatschny für ihre Zusage zur Teilnahme an der Prüfungskommission meiner Dissertation.

Besonderer Dank gilt der Arbeitsgruppe aus Augsburg für die tolle gemeinsame Zusammenarbeit im Bereich der Halluntersuchungen. Dabei möchte ich insbesondere Prof. Dr. Philipp Gegenwart für die konstruktiven Diskussionen danken und die Bereitschaft den weiten Weg auf sich zu nehmen, um am Tag der Disputation Teil meiner Prüfungskommission sein zu kön- 
nen. Ebenfalls bedanke ich mich bei Robert Gruhl für die Anfertigung der TEM-Aufnahmen und bei Ludwig Scheuchenpflug für die Durchführung der Hallmessungen. Es hat mir viel Freude bereitet zusammen mit euch in das Gebiet der Skyrmionen einzutauchen.

Mein Dank geht auch an Dr. Vladimir Roddatis für die zahlreichen und aufwendigen TEM-Aufnahmen und die Unterstützung im Rahmen der DFG-Projekte MO-2255-4 und RO-5387/3-1.

Lange hat er unser Institut schon verlassen und dennoch möchte ich mich ausdrücklich bei meinem ehemaligen Bürokollegen Christoph Meyer bedanken. Zu Beginn meiner Zeit als Doktorand warst Du mir eine große Stütze und ein unglaublich guter Ansprechpartner bei fast allen Fragen. Selbst nachdem Du nach Jahren das Institut schon verlassen hast, haben wir zusammen bis spät in die Nacht miteinander telefoniert, um einige Paper fertig zu bringen. Das alles hat mir immer große Freude bereitet.

Ein mindestens genauso bedeutungsvoller Wegbegleiter ist mein Doktorbruder Vitaly Bruchmann-Bamberg. Von Beginn an saßen wir beide im selben Boot. Das gab mir oft das Gefühl mit meinen Problemen nicht allein zu sein. Es tat immer gut, in dein Büro zu kommen und all meinen Frust über die MAD, den Chef oder die Welt auszulassen. Es ist nach all den Jahren noch immer bewundernswert mit welcher Selbstlosigkeit Du immer ,ja" sagst, wenn jemand um Hilfe bittet. Dabei ist es ganz egal, ob Studierender, Techniker, jemand aus einem ganz anderen Institut - jeder weiß, dass er bei dir immer Rat und Lösung findet. Das spiegelt sich insbesondere darin wider, dass es kaum eine Danksagung gibt, in der Du nicht vorkommst. So auch in meiner. Danke für deine endlose Hilfsbereitschaft.

Ebenfalls geht mein Dank an meine zwei weiteren Mitdoktoranden Karen Stroh und Leonard Schüler. Dabei will ich nicht unter den Tisch fallen lassen, dass ich stolz darauf bin, dass es mein ehemaliger Bachelorstudent zum Doktorand geschafft hat. Ich drücke euch beiden auch weiterhin die Daumen, dass die Versuche alle rund laufen und dass Vasily mit seinen zahlreichen Ideen euch nicht zu sehr in die Mangel nimmt. 
Für die tolle Atmosphäre möchte ich natürlich auch dem gesamten I. Physikalischen Institut danken. In den fünf Jahren habe ich tatsächlich viele Leute kommen und gehen gesehen. Dennoch hat das Institut weiterhin eine besondere und lebhafte Atmosphäre, die hoffentlich noch lange erhalten bleibt. Dabei möchte ich mich ganz herzlich bei dem Team der Techniker bedanken. Hartmut Eichenberg und Bastian Beyland für die zuverlässige Unterstützung, wenn es mal ganz schnell darum ging „schon gestern” etwas für die MAD zu bauen. Uta Filippich, Kai Dörner, Simon Stoldt, Finn Krummacker und Michaela Mecke für die Unterstützung bei den tausend Dingen, die im Arbeitsalltag so angefallen sind und dafür, dass ihr dem Institut diese besondere Note verleiht. Dabei möchte ich ganz lieb Carsten Mahn hervorheben, der sich beim Essen oder Grillen immer wieder meine pseudomoralischen Reden über Klimawandel und Autos antun musste.

Ich möchte mich insgesamt bei all meinen Freunden und jenen, die mich als solchen bezeichnen, bedanken. In Zeiten einer unendlich schnell voranschreitenden Digitalisierung und der Pandemie wird mir euer Wert immer wieder bewusst. Ihr macht mich zu dem, der ich bin und mein Leben ein ganzes Stück bunter! Danke euch allen dafür.

Der wohl größte Dank geht an meine Eltern Barbara und Alois Ksoll, sowie an meine Babcia Julia Puk und Oma Anne-Marie Ksoll. To Wy umožliwiłiście to wszystko w pierwszej kolejności! W czasach szkolnych, a takz̊e podczas studiów mogłem się rozwijać dzięki Wam i odnaleźć swoją indywidualną drogę. Dziękuję za to i z̊e zawsze jesteście przy mnie.

So, das war's dann fast schon. Ich habe mir lange überlegt, dass ich diese Arbeit verbunden mit dem gesamten Lebensabschnitt den kommenden Generationen widmen möchte. Auf dass mir die gewonnenen Erfahrungen die Stärke und Weisheit geben, die richtigen Entscheidungen fällen und Taten vollbringen zu können. Damit auch unsere Kinder in einer wahrhaft gesunden, bunten und gedeihlichen Welt aufwachsen können, wie ich es tat. 



\section{Lebenslauf}




\section{Publikationen}

\section{Artikel in wissenschaftlichen Fachzeitschriften}

1. C. Meyer, V. Roddatis, P. Ksoll, B. Damaschke, V. Moshnyaga, Structure, magnetism, and spin-phonon coupling in heteroepitaxial $\mathrm{La}_{2} \mathrm{CoMnO}_{6} / \mathrm{Al}_{2} \mathrm{O}_{3}$ (0001) films, Phys. Rev. B 98, 134433, 2018. DOI: https://doi.org/10.1103/PhysRevB.98.134433

2. R. Mandal, M. Chandra, V. Roddatis, P. Ksoll, M. Tripathi, R. Rawat, R. J. Choudhary, V. Moshnyaga, Magneto-dielectric effect in relaxor superparaelectric $\mathrm{Tb}_{2} \mathrm{CoMnO}_{6}$ film, Phys. Rev. B 101, 094426, 2020.

DOI: https://doi.org/10.1103/PhysRevB.101.094426

3. P. Ksoll, R. Mandal, C. Meyer, L. Schüler, V. Roddatis, V. Moshnyaga, Emergent Double Perovskite Phase at LaMnO $\mathrm{O}_{3} / \mathrm{LaNiO}_{3}$ Interfaces: Coupled Charge Transfer and Structural Reconstruction, Phys. Rev. B 103, 195120, 2021.

DOI: https://doi.org/10.1103/PhysRevB.103.195120

4. C. Meyer, P. Ksoll, V. Roddatis, V. Moshnyaga, Spin-Phonon Coupling in $A_{2} \mathrm{BMnO}_{6}(A=L a, \operatorname{Pr}, \mathrm{Nd}, \mathrm{Sm}, \mathrm{Gd} ; \mathrm{B}=\mathrm{Co}$, Ni) DoublePerovskite Thin Films: Impact of the A-Site Cation Radius, Crystals 11, 747, 2021.

DOI: https://doi.org/10.3390/cryst11070747

5. P. Ksoll, C. Meyer, L. Schüler, V. Roddatis, V. Moshnyaga, B-site Cation Ordering in Films, Superlattices and Layer-by-Layer Grown Double Perovskites, Crystals 11, 734, 2021 (invided review paper).

DOI: https://doi.org/10.3390/cryst11070734 


\section{Beiträge zu Konferenzen}

1. Frühjahrstagung der DPG, Dresden, 2017, Postertitel:

Growth of oxide microstructures by metalorganic aerosol deposition technique

2. Raman Scattering in Advanced Materials Symposium, Braunschweig, 2017

3. Frühjahrstagung der DPG, Berlin, 2018, Postertitel:

B-site Ordering in $\mathrm{La}_{2} \mathrm{CoMnO}_{6}$ Films: Influence of Lattice Strain

4. Frühjahrstagung der DPG, Regensburg, 2019. Postertitel:

Controlling the B-site Ordering by a Layer-by-Layer MAD growth

5. Frühjahrstagung der DPG, Dresden, 2020 (Pandemie bedingt abgesagt). Vortragstitel:

Emergent Double Perovskite Phase at $\mathrm{LaMnO}_{3} / \mathrm{LaNiO}_{3}$ Interfaces

6. The International Magnetics Conference 2020, Montréal, Kanada (Pandemie bedingt abgesagt). Postertitel:

Emergent Double Perovskite Phase at $\mathrm{LaMnO}_{3} / \mathrm{LaNiO}_{3}$ Interfaces 